\title{
Solution Phase Syntheses of Nanoparticles and Nanowires
}

\author{
by \\ Teck Hock Lim
}

\author{
A thesis \\ submitted to the Victoria University of Wellington \\ in fulfilment of the \\ requirements for the degree of \\ Doctor of Philosophy \\ in Chemistry
}

Victoria University of Wellington

April 2008 


\section{Abstract}

This thesis is concerned with the preparation of metal and semiconductor nanostructures in solution, specifically bismuth and indium metal nanoparticles, gallium nitride nanoparticles, indium phosphide nanowires and zinc phosphide nanoparticles. There were two aims: firstly to study if gallium nitride nanoparticles with improved crystallinity and size distribution could be synthesized and secondly to find and develop new methods to prepare crystalline indium phosphide nanowires and zinc phosphide nanoparticles using precursors that are safe and cheap. The crystallinity, structures, morphologies and chemical compositions of the nanostructures synthesized in this thesis were studied primarily by transmission electron microscopy (TEM), powder X-ray diffraction (PXRD) and energy dispersive X-ray spectrometry (EDS).

For the synthesis of gallium nitride, two approaches were taken. The first revolves around the direct metathesis reaction between gallium trichloride and lithium nitride under ambient pressure. A range of solvents with different polarities has been tested and only in highly polar solvents crystalline nanostructures were produced. These crystalline nanostructures however are not of gallium nitride.

The second approach involves thermally decomposing an organometallic precursor. Organometallic compounds $\left[\mathrm{Ga}_{2}\left(\mathrm{NMe}_{2}\right)_{6}\right]$ (compound 1) and $\left[\left(\mathrm{Me}_{3} \mathrm{C}\right)_{2} \mathrm{Ga}\left(\mu-\mathrm{NHNHCMe}_{3}\right)\right]$ (compound 2) were chosen from the literature as precursors. Compound 1 was synthesized in a very small yield together with by-products. Thermal decomposition of the mixture produced no nanoparticles. A compound (compound S2) which is structurally similar to compound 2 was successfully synthesized and was subjected to thermal decomposition in ammonia to produce crystalline monodispersed nanoparticles. However, these nanoparticles could not be confidently identified as gallium nitride.

The outcome from the reaction of lithium borohydride and indium trichloride was found to be strongly solvent dependent. In toluene a white precipitate was obtained. Both in isobutylamine and $\mathrm{N}, \mathrm{N}$-diethylaniline indium metal nanoparticles were produced as black solutions. Only in isobutylamine, small monodispersed indium nanoparticles can be 
produced. The isobutylamine method was extended to prepare bismuth metal nanoparticles. However, the bismuth nanoparticles prepared were moderately polydispersed in size.

Two new methods were developed to prepare indium phosphide nanowires from red phosphorus and phosphorus pentabromide via Solution-Liquid-Solid growth. Borohydride reagents are required in both methods to produce chemically active intermediates which further react to form indium phosphide nanowires in the presence of pre-synthesized indium metal or bismuth nanoparticles.

The diameter of indium phosphide nanowires prepared from red phosphorus depends strongly on the reaction sequences. If indium metal nanoparticles are formed prior to the addition of red phosphorus, large nanowires $(>300 \mathrm{~nm})$ are produced. Reversing the sequences, small nanowires $(50-100 \mathrm{~nm})$ are produced. Red phosphorus residue remains in the products regardless of the reaction sequences and is difficult to remove completely by chemical means.

The reaction which employs phosphorus pentabromide as precursor proceeds via intermediates of hydrogen phosphide and indium metal to form indium phosphide. The reaction temperature dictates the crystallinity of the product and needs to be $>170{ }^{\circ} \mathrm{C}$ to produce crystalline indium phosphide. The way hydrogen phosphide is introduced to the reaction and the presence or absence of pre-synthesized metal seeds together control the morphology of indium phosphide synthesized. The best set of conditions established in this thesis allows the preparation of indium phosphide in $\sim 100 \%$ nanowire morphology.

The hydrogen phosphide method was adapted to produce zinc phosphide nanoparticles. The choice of the reaction solvent was found to be most critical. Amorphous particles were produced in trioctylphosphine at as high as $330{ }^{\circ} \mathrm{C}$ whereas in oleylamine and N,Ndiethylaniline crystalline zinc phosphide $\left(\alpha-\mathrm{Zn}_{3} \mathrm{P}_{2}\right)$ nanoparticles were produced at $\sim 200{ }^{\circ} \mathrm{C}$.

An overall conclusion is given in the last chapter comparing the methods developed in this thesis with literature methods paying particular foci on the level of hazard and the costs of the chemical reagents involved. 


\section{Acknowledgements}

The past three years have been challenging and interesting and I have learned a lot both about science and non-science. There is a long list of people to whom I owe a big thankyou for without them this thesis would not have been possible. In particular, I would like to thank:

My supervisor Dr. Richard D. Tilley for his encouragement, forbearance, advice and for introducing me to the very beautiful world of electron microscopy.

My co-supervisor Professor Pablo Etchegoin for his encouragement, wisdom, good humour and for his help in optical experiments.

Dr. Christopher Bumby for his enthusiasm, advice, his teaching me optical physics and for some of his mind-stimulating "crazy" ideas of nanoparticle synthesis.

David Flynn for his help in SEM and for fixing the TEM especially when it went out of order due to the mysterious "Teck's effect".

Professor Richard J. D. Tilley for valuable discussions and his kind wishes.

Dr. Eric Le Ru for his help in optical experiments.

My other colleagues within the Tilley group for their company and help.

The "physicists" Shrividya Ravi, Dmitri Schebarchov and Matthias Meyer for being great colleagues and friends.

Almas Zayya for being a good friend and for proof reading some of this thesis.

Dr. Gordon Heeley, Dr. Kirsten Edgar, Dr. Christopher Bumby for proof reading some of this thesis. 
My family in Malaysia in particular my parents for their patience, understanding and unconditional support in the "strange business" that is my doctoral research.

Lastly I would like to thank the New Zealand International Doctoral Research Scholarship and Curtis-Gordon Scholarship in Chemistry for funding and financial support. 


\section{Table of Contents}

Abstract

Acknowledgements $\quad$ iv

Table of Contents $\quad$ vi

$\begin{array}{ll}\text { Abbreviations } & \text { xi }\end{array}$

$\begin{array}{ll}\text { Chapter } 1 \text { Introduction } & 1\end{array}$

1.1 General Overview 1

1.2 Solution Phase Synthesis of Nanoparticles 2

1.21 Thermodynamic Considerations 2

1.3 Mechanisms of Metal Nanoparticles Seeded Growth of $\quad 4$

Semiconductor Nanowires

1.31 Vapour Phase Synthesis via Vapour-Liquid-Solid Growth 4

1.32 Solution Phase Synthesis via Solution-Liquid-Solid Growth 6

1.4 Quantum Confinement in Semiconductor Nanoparticles and Nanowires 7

1.5 Material Systems: Semiconductors and Metals 7

1.51 Gallium Nitride $\quad 8$

1.52 Indium Phosphide $\quad 11$

1.53 Zinc Phosphide $\quad 13$

1.54 Bismuth Metal and Indium Metal 17

$\begin{array}{ll}1.6 \text { References } & 18\end{array}$

Chapter 2 General Preparative Methodology and Characterization Techniques 24

2.1 Solution Phase Synthesis of Semiconductors and Metal Nanomaterials 24

2.2 Sample Characterization 26

2.3 Data Interpretation 28

2.31 High Resolution Transmission Electron Microscopy 28

2.32 Energy Dispersive X-ray Spectrometry 30

2.33 Selected Area Electron Diffraction 31

2.4 Fundamental of High Resolution Transmission Electron Microscopy 31

2.5 Fundamental of Energy Dispersive X-ray Spectrometry 35 
Chapter 3 Attempted Syntheses of Crystalline Gallium Nitride Nanoparticles

3.1 Metathesis of Gallium Trichloride and Lithium Nitride 44

3.11 Reaction of Gallium Trichloride and Lithium Nitride in Benzene 45

3.12 Reaction of Gallium Trichloride and Lithium Nitride in the Presence of 12-Crown-4 (1,4,7,10-Tetraoxacyclododecane) 46

3.13 Testing Dibenzo-18-Crown-6 (2,3,11,12-Dibenzo-1,4,7,10,13,16Hexaoxacyclooctadeca-2,11-Diene) As Solvent

3.14 Reaction of Gallium Trichloride and Lithium Nitride in Trioctylamine

3.15 Reaction of Gallium Trichloride and Lithium Nitride in Hexadecylamine

3.16 Reaction of Gallium Trichloride and Lithium Nitride in Trioctylamine/

Hexadecylamine Mixture 52

3.17 Reaction of Gallium Trichloride and Lithium Nitride in Dibenzofuran

3.18 Reaction of Gallium Trichloride and Lithium Nitride in Octadecene/

Dibenzofuran Mixture $\quad 54$

3.19 Testing Ammonium Chloride as Solvent 54

3.20 Reaction of Gallium Trichloride and Lithium Nitride in Molten

Lithium Chloride/Potassium Chloride Eutectic Mixture

3.3 Thermal Decomposition of Organometallic Precursors Containing Ga-N Bonds

3.31 Synthesis and Thermal Decomposition of

Bis[di(tert-butyl)gallium (tert-butyl-hydrazide)]

3.32 Attempted Synthesis and Thermal Decomposition of 


\section{Chapter 4 Solution Synthesis of Indium Phosphide Nanowires Using}

\section{Red Phosphorus}

4.1 Reaction of Indium Acetate and Red Phosphorus: The Formation of Indium Oxide

4.2 Reaction of Indium Trichloride and Red Phosphorus in

Trioctylphosphine Oxide/ Methyl-Myristate Mixture

4.3 Reactions of Indium Trichloride, Red Phosphorus and Sodium Borohydride

4.4 Reactions of Indium Trichloride, Red Phosphorus and Sodium Borohydride

4.5 Discussion

4.6 Conclusions

4.7 Experimental

4.8 References

Chapter 5 Syntheses of Indium and Bismuth Nanoparticles

5.1 Indium Metal Nanoparticles

5.11 Reaction of Indium Trichloride and Lithium Borohydride in Toluene

5.12 Reaction of Indium Trichloride and Lithium Aluminium Hydride in Toluene

5.13 Reaction of Indium Trichloride and Lithium Borohydride in Isobutylamine

5.14 Reaction of Indium Trichloride and Lithium Borohydride in

N,N-diethylaniline

5.2 Reaction of Bismuth Nitrate Pentahydrate and Lithium Borohydride in Isobutylamine

5.4 Conclusions 
Chapter 6 Solution Synthesis of Indium Phosphide Nanowires: Indium

Trichloride and Phosphorus Pentabromide as Precursors 126

6.1 Reaction of Lithium Borohydride and Phosphorus Pentabromide 131

6.2 Reaction of Indium Trichloride and Phosphorus Pentabromide 133

6.3 Reaction of Indium Trichloride, Lithium Borohydride and

Phosphorus Pentabromide

134

6.31 Hot Injection of A Pre-reacted Mixture of Indium Trichloride,

$\begin{array}{ll}\text { Phosphorus Pentabromide and Lithium Borohydride } & 135\end{array}$

6.32 Reactions of Indium Trichloride, Hydrogen Phosphide and

Lithium Borohydride

6.33 Injection of A Mixture of Indium Trichloride, Phosphorus Pentabromide and Lithium Borohydride to Trioctylphosphine

6.34 Indium Trichloride and Indium Metal Seeds in N,N-diethylaniline

Treated with Hydrogen Phosphide and Lithium Borohydride

6.35 A Mixture of Indium Trichloride, Indium Metal Seeds, Phosphorus

Pentabromide and Lithium Borohydride in Trioctylphosphine Heated in

An Hot Oil Bath

6.36 A Mixture of Indium Trichloride and Phosphorus Pentabromide in

Trioctylphosphine Treated with A Mixture of Lithium Borohydride

and Indium Metal Seeds

6.37 Indium Trichloride in Trioctylphosphine Treated with Phosphorus

Pentabromide and Lithium Borohydride

6.38 A Mixture of Indium Trichloride and Pre-synthesized

Indium Metal Seeds Treated with Phosphorus Pentabromide

and Lithium Borohydride

152

6.39 Indium Trichloride in Trioctylphosphine Treated with A Mixture

of Bismuth Metal Nanoparticles, Hydrogen Phosphide and

Lithium Borohydride

156

6.4 Discussion

6.5 Conclusions

6.6 Experimental 165

$\begin{array}{ll}6.7 \text { References } & 177\end{array}$ 
7.1 Reaction of Zinc Chloride and Lithium Borohydride in Oleylamine 183

7.2 Reaction of Zinc Chloride and Lithium Borohydride in N,N-diethylaniline

7.3 Reactions of Zinc Chloride, Lithium Borohydride and Phosphorus

Pentabromide in Trioctylphosphine

7.4 Reaction of Zinc Chloride, Lithium Borohydride and Phosphorus

Pentabromide in N,N-diethylaniline

7.5 Reaction of Zinc Chloride, Lithium Borohydride and Phosphorus

Pentabromide in Oleylamine

188

7.6 Discussion

7.7 Conclusions

7.8 Experimental

200

7.9 References

Chapter 8 Overall Conclusions and Future Work

205

8.1 Attempted Synthesis of Gallium Nitride Nanoparticles

205

8.2 Preparation of Indium Phosphide Nanowires from Red Phosphorus

8.3 Formation of Indium Phosphide Nanowires from Phosphorus

Pentabromide via Hydrogen Phosphide

8.4 Formation of Zinc Phosphide Nanoparticles From Phosphorus

Pentabromide via Hydrogen Phosphide

8.5 Indium Metal Nanoparticles and Bismuth Metal Nanoparticles in Isobutylamine

8.51 Comparison to Literature Methods 


\section{Abbreviations}

\begin{tabular}{|c|c|}
\hline ED & Electron Diffraction \\
\hline EDS & Energy Dispersive X-ray Spectrometry \\
\hline FESEM & Field Emission Scanning Electron Microscope \\
\hline FFT & Fast Fourier Transform \\
\hline HRTEM & High Resolution Transmission Electron Microscopy / or \\
\hline & High Resolution Transmission Electron Microscope \\
\hline $\mathrm{h}$ & hours \\
\hline IR & Infrared \\
\hline LCG & Laser Assisted Catalytic Growth \\
\hline LED & Light Emitting Diode \\
\hline $\mathrm{MBE}$ & Molecular Beam Epitaxy \\
\hline MOCVD & Metalorganic Chemical Vapour Deposition \\
\hline NMR & Nuclear Magnetic Resonance \\
\hline PL & Photoluminescence \\
\hline PXRD & Powder X-ray Diffraction \\
\hline SAED & Selected Area Electron Diffraction \\
\hline SEM & Scanning Electron Microscopy \\
\hline SLS & Solution-Liquid-Solid \\
\hline TEM & Transmission Electron Microscopy or \\
\hline & Transmission Electron Microscope \\
\hline VLS & Vapour-Liquid-Solid \\
\hline UV-VIS & Ultra-Violet-Visible \\
\hline
\end{tabular}




\section{Abbreviations (for NMR data)}

$\mathrm{Me}$

Et

${ }^{\mathrm{t}} \mathrm{Bu}$

$\mathrm{Ph}$

S

br

\section{Synonyms}

Myristic acid

Indium(myristate) $)_{3}$

Oleylamine
Methyl group

Ethyl group

Tert-butyl group

Phenyl group

singlet

broad
Tetradecanoic acid

Indium(tetradecanoate) ${ }_{3}$

cis-1-Amino-9-octadecene 


\section{Chapter 1 Introduction}

\subsection{General Overview}

The research work presented in this thesis aimed at finding and developing new solution phase syntheses of metal nanoparticles, semiconductor nanoparticles and semiconductor nanowires. The main motivation was to investigate novel synthetic methodologies with the use of reagents which are more common or safer than those employed in literature. The nanostructures were studied using high resolution transmission electron microscopy (HRTEM) as the main tool and in the case of nanowires, investigations were conducted to ascertain the presence of crystallographic defects and nanowire growth mechanism. It was also intended to study the effects of reaction parameters on the sizes and morphologies of the nanostructures. The materials that were studied included indium metal, bismuth metal, gallium nitride, indium phosphide and zinc phosphide. The metals were researched mainly for their uses in the fabrication of semiconductor nanowires in solution. The semiconductor materials were of interest because of their potential applications as described in section 1.5.

The results of this thesis are divided into seven chapters. Chapter 2 discusses the general preparation of materials and the characterization techniques used to analyse the structures, chemical compositions and optical properties of the materials investigated. Chapter 3 describes research work on attempted syntheses of gallium nitride nanoparticles. Chapter 4 is on the preparation and characterization of indium phosphide nanowires formed from red phosphorus. In chapter 5, the preparation and characterization of indium and bismuth metal nanoparticles are described. Chapter 6 details the synthesis and characterization of indium phosphide nanowires prepared from phosphorus pentabromide. Chapter 7 is concerned with adapting the method developed in chapter 6 to produce zinc phosphide nanoparticles. 


\subsection{Solution Phase Synthesis of Nanoparticles}

Crystalline inorganic nanostructures may be prepared from either vapour phase and solution phase techniques. The preparation methods described in this thesis are concerned with only solution phase techniques. Vapour phase techniques such as molecular beam epitaxy (MBE) and metal organic chemical vapour deposition (MOCVD) generally produced nanostructures on substrates. ${ }^{1}$ There exists a limit on the physical size of a substrate and the number of substrates that may be processed during one single batch. Therefore, the throughput of the process is limited. Solution phase approach may offer a solution to this limitation as there are no substrate issues. The nanostructures produced are free-standing and exist as colloids in solution.

Solution phase synthesis in general involves reacting atomic or molecular species in a solvent/surfactant mixture. The solvent/surfactant mixture serves as a reaction medium in which reaction, nucleation and crystal growth occur. The surfactant is often an organic molecule which interacts dynamically with the surface of the growing nanostructures thus regulating the size and shape of the final nanostructures. ${ }^{1}$

There exits a great many accounts on nanoparticles and nanowires as the field of nanoparticle and nanowire syntheses advanced at a fast pace. The following aim to provide a conceptual and brief discussion on the thermodynamic considerations of the formation of crystalline nanoparticles.

\subsection{Solution Phase Synthesis of Crystalline Nanoparticles : Thermodynamic Considerations}

The following discussion is closely related to the account by O'Connor and coworkers. ${ }^{2}$

\section{Nucleation, Crystal Growth, and Growth Termination and Stabilization}

Theoretically, in any liquid phase synthesis of crystalline nanoparticles, there are three steps to consider: (i) nucleation of a sparingly soluble product under supersaturation conditions; (ii) growth of nuclei into crystalline nanoparticles and (iii) growth termination and nanoparticles stabilization. In theory, control in the size and shape distribution of nanoparticles prepared in solution can be achieved via the capability to 
temporally separate the nucleation process from the growth process. The growth process can be either reaction-limited or diffusion-limited. If the nucleation process is made immediate, which is referred to as a "burst" of nucleation, the growth process is in a large degree diffusion-limited. Temporal separation of the nucleation process from the growth process can be achieved by manipulating reaction temperature and concentration gradients.

Nanoparticles formed in solution have a high surface to volume ratio and therefore a high surface energy. Such system is not thermodynamically stable and the nanoparticles have a strong propensity to agglomerate in order to achieve a minimal surface energy. In general, such agglomeration process can be prevented or controlled by means of steric repulsion and electrostatic repulsion. A detailed demonstration on the physical chemistry of colloidal systems is given by Everett. ${ }^{21}$

Steric stabilization in colloidal systems arises from the mutual repulsion between capping ligands on the surface of nanoparticles (surfactants, polymers or other organic species bound to the nanoparticles). Typical capping ligands have one lyophilic group and one lyophobic and are classified as ionic or neutral. The most common ionic ligands contain oxygen-donor and/or sulfur-donor in the forms of carboxylate, phosphonate, alkoxide and thiolate. Common neutral ligands include amines, phosphines, phosphine oxides and alcohols. These neutral ligands often have long alkyl chains and it is the interaction among these long alkyl chains that the agglomeration of nanoparticles is prevented. A few examples of these neutral ligands are hexadecylamine, trioctylamine, trioctylphosphine, trioctylphosphine oxide and octanol.

\section{Roles of Capping Ligand and Steric Stabilization}

The roles of capping ligands include (i) interacting with metal ions in solution and the surface of nanoparticles therefore affecting the equilibrium and the rate of nucleation and growth leading to size and shape control of the nanoparticles; (ii) stabilizing nanoparticles in solution by preventing agglomeration by means of steric repulsion; (3) affecting the optoelectronic and magnetic properties of the nanoparticles due to their own electronic and binding properties, such as functioning as a passivating agent allowing an increase of the photoluminescence efficiency and (iv) offering chemical protection from oxidation and (v) rendering the nanoparticles solubilities in solvents. 


\section{Electrostatic Stabilization}

Electrostatic stabilization (van der Waals stabilization) arises as a result of mutual repulsion between layers of charge species chemically absorbed on the surface of nanoparticles. The mechanism of van der Waals stabilization involves absorption of charged species and possibly solvent molecules forming an electric double layer. Same charge repulsion acts as a strong stabilization force resulting in stable colloids.

\subsection{Mechanisms of Metal Seeded Growth of Semiconductor Nanowires}

\subsection{Vapour Phase Synthesis via Vapour-Liquid-Solid Growth}

Vapour-liquid-solid (VLS) growth was first proposed in the year 1964 to explain the formation of silicon nanowires grown by the high temperature reduction of silicon tetrachloride in the presence of metal nanoparticles. ${ }^{3}$ As may be seen in figure 1.31a, an alloy is formed from a metal nanoparticle (or "seed") and reactant species (silicon atoms from the reduction reaction) when the VLS growth commences. The alloy fully melts into a liquid droplet at the eutectic composition. The liquid droplet then serves as a preferential site for the absorption of reactant species and upon supersaturation reactant species nucleates in the liquid droplet. Incoming reactant species preferentially condenses onto the minute nuclei present in the droplet instead of forming another nucleus in the droplet in order to minimize the total surface energy. Axial growth commences as more reactant species condenses onto the growing nuclei and eventually a solid nanowire is formed. The liquid droplet freezes into a solid when being cooled and excess reactant species crystallizes out bridging the nanowire with the now solid metal nanoparticle. 

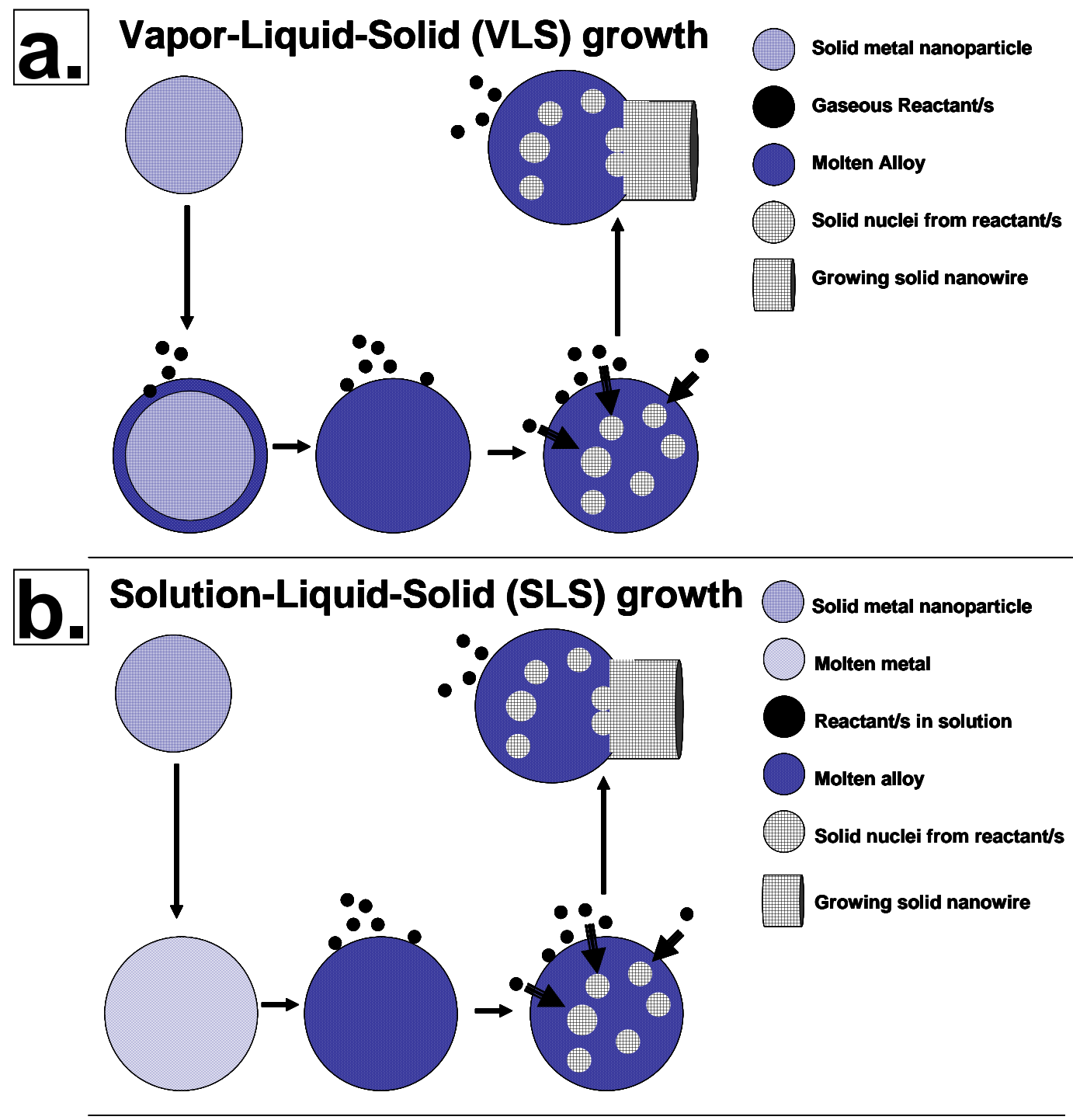

Figure 1.31 Schematic diagrams of VLS and SLS growth

A few key features of VLS grown nanowires include: (i) the diameter of the nanowires grown are controlled by the size (diameter) of the metal nanoparticles; (ii) the nanowires often have spherical seed nanoparticles attached to their tips; (iii) the length of the nanowires can be controlled by controlling the supply of reagents; (iv) intended selective dopings of nanowires could be achieved by supplying dopants during the growth of nanowires. 


\subsection{Solution Phase Synthesis via Solution-Liquid-Solid Growth}

Solution-liquid-solid (SLS) growth was first proposed by Trentler and coworkers in 1996 as an analogy of VLS growth. ${ }^{4,5}$ In their study, indium phosphide nanowires were synthesized by thermally decomposing in solution organometallic compounds of indium. The indium phosphide nanowires have spherical indium metal nanoparticles attached to their tips. The appearance of the indium phosphide nanowires was very similar to the characteristic appearance of VLS grown nanowires. As may be seen in figure $1.31 \mathrm{~b}$ a molten metal droplet forms at a reaction temperature close to or higher than its melting point. The liquid droplet dissolves reactant species and forms a liquid alloy droplet. Upon supersaturation, reactant species crystallizes out as minute nuclei. More reactant species then condenses onto the nuclei and axial growth commences. Prolonged growth then leads to the formation of nanowires. VLS and SLS growths differ from each other in that in SLS growth molten metal droplets are formed prior to alloy formation.

In its early state SLS technique produced nanowires with bend and kinks and with broad diameter and length distributions. Long straight indium phosphide nanowires with uniform diameters could now be prepared by SLS growth as the method has matured. ${ }^{6,7,8}$ One important advance made was the use of pre-synthesized monodisperse nanoparticles of metals with low melting points for examples indium, gallium and bismuth as seeds. $^{7}$ 


\subsection{Quantum Confinement in Semiconductor Nanoparticles and Nanowires}

The strongly size-dependent optical properties of semiconductor nanoparticles are welldocumented in the literature. ${ }^{9-12}$ This section aims to introduce succinctly the quantum confinement effect observed in semiconductor nanoparticles and nanowires.

The band gap of a semiconductor can be viewed as the energy gap between the highest level of the valence band and the lowest level of the conduction band. After an electron is promoted to the conduction band a hole is created in the valence band. This electronhole pair is regarded as an exciton. If the electron and hole form a bound state, the electron orbits the hole at a certain distance as in the Bohr's model of the hydrogen atom. This distance, termed Bohr radius, is material sensitive. For examples, the Bohr radius is $\sim 2.5 \mathrm{~nm}$ for gallium nitride ${ }^{13}$ and $\sim 20 \mathrm{~nm}$ for indium phosphide ${ }^{14}$. When the physical size of a semiconductor particle is reduced to be smaller than its Bohr radius, the exciton becomes spatially confined and the confinement results in an increase in the band gap (a blue shift of the band gap). In general, the blue shift in the band gap becomes significant when the particle size is reduced to be in between 1 to $10 \mathrm{~nm}$. Semiconductor particles of size $<10 \mathrm{~nm}$ are often called quantum dots and therefore the effect of spatially confining an exciton in a semiconductor nanoparticle is termed the quantum confinement effect.

For nanowires, the quantum confinement occurs anisotropically along the radial direction. ${ }^{14}$ When comparing a nanowire and a spherical nanoparticle both having the same diameter, the quantum confinement effect is found weaker in the nanowire. ${ }^{15}$ 


\subsection{Material Systems: Semiconductors and Metals}

All crystal structures presented in this section unless otherwise referenced were calculated using CrystalMaker 2.0 Demonstration version.

\subsection{Gallium Nitride (GaN)}

Gallium nitride $(\mathrm{GaN})$ is a good candidate for opto-electronic applications particularly in the field of blue light emitting devices (LEDs) ${ }^{16}$ and blue laser diodes ${ }^{17}$. Gallium nitride-based LEDs have been used in commercial LEDs since $1990 .{ }^{18}$ While gallium nitride is not the only promising candidate for opto-electronic devices requiring blue or UV light emission or absorption, gallium nitride displays high chemical inertness and radiation resistance. ${ }^{19}$ Gallium nitride also has great potential in functioning as a photocatalyst in a photochemical fuel cell. ${ }^{20-23}$ Recently, the use of n-type gallium nitride photoelectrode in an electrolyte to generate hydrogen gas from water-splitting under UV light were reported. ${ }^{24}$

\section{Crystal Structures and Physical Properties}

Gallium nitride can crystallize in either wurtzite (hexagonal) or zinc blende (face centred cubic) structures (figure 1.510 and 1.511). In the wurtzite structure, gallium atoms form a primitive hexagonal cell and on top of each gallium atom there is a nitrogen atom. In the zinc blende structure, gallium atoms form a face centred cubic cell and nitrogen atoms occupy half of the tetrahedral sites available. The wurtzite phase is thermodynamically most stable and is the most common phase under ambient conditions. The band structures of both wurtzite and zinc blende gallium nitride are presented in figure 1.512. Both wurtzite and zinc blende gallium nitride are direct gap semiconductors with room temperature $\Gamma$ transitions of $3.39 \mathrm{eV}$ and $3.2 \mathrm{eV}$ respectively. ${ }^{16 a, 25}$ The Bohr radius of gallium nitride has been estimated to be $2.5 \mathrm{~nm} .{ }^{16 \mathrm{a}}$ The basic unit cell parameters, band gaps and exciton Bohr radii of both wurtzite and zinc blende gallium nitride are shown in table 1.513. 

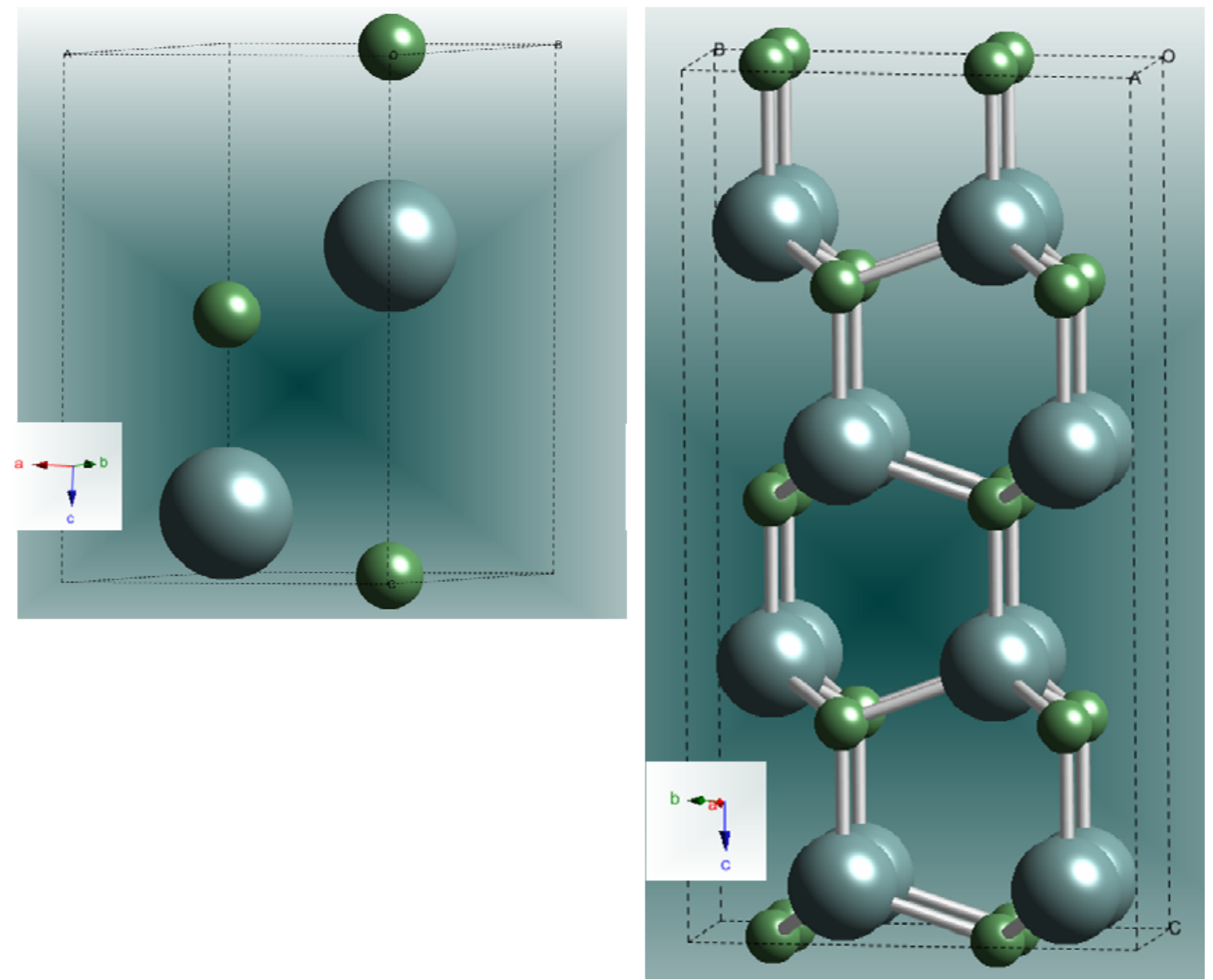

Figure 1.510 A sphere packing model of the hexagonal unit cell of gallium nitride and a ball-and-stick ( $2 \times 2 \times 2$ unit cells) presentation to illustrate the hexagonal symmetry of the crystal structure. Green atoms represent gallium atoms and blue atoms represent nitrogen atoms.

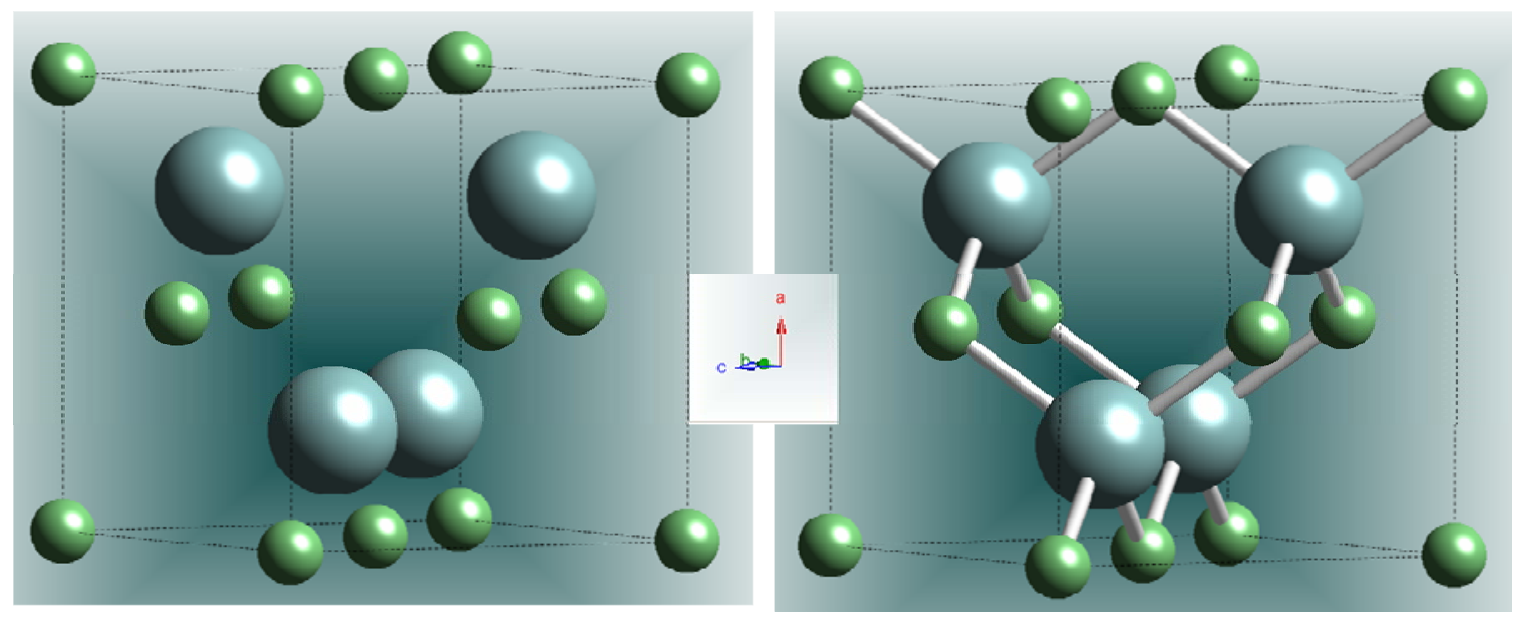

Figure 1.511 A sphere packing model of the face centred cubic unit cell of gallium nitride and a ball-and-stick presentation shows the nitrogen atoms at the tetrahedral sites. 


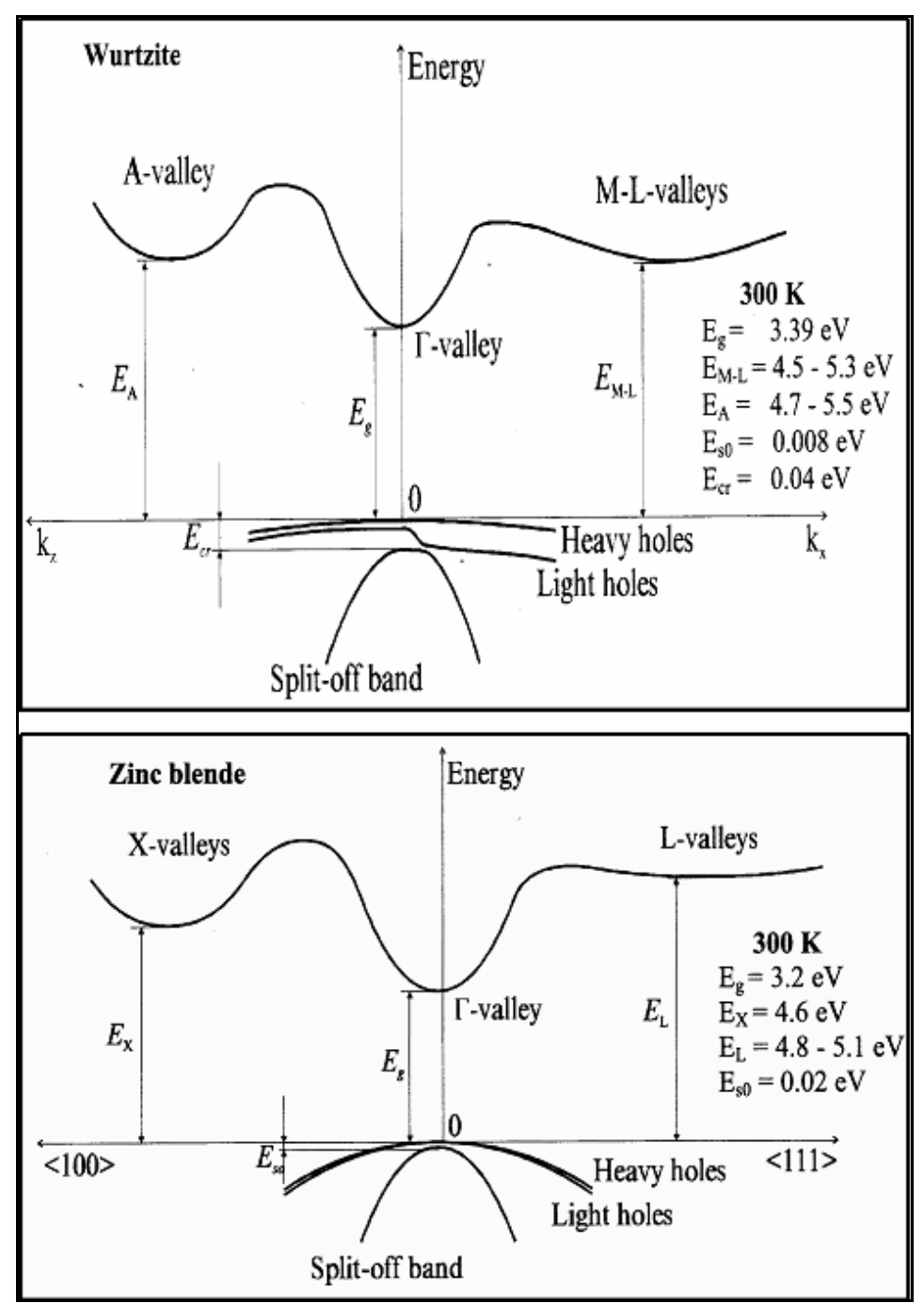

Figure 1.512 Band structures of hexagonal (wurtzite) gallium nitride and cubic (zinc blende) gallium nitride. ${ }^{25}$

Table 1.513 Basic parameters of gallium nitride.

\begin{tabular}{|c|c|c|}
\hline Structure & Zinc Blende & Wurtzite \\
\hline Thermodynamic Stability & Metastable & Stable \\
\hline Lattice Parameter at $300 \mathrm{~K}$ & $\mathrm{a}=4.503 \AA^{26}$ & $\begin{array}{c}\mathrm{a}=3.1891 \AA^{27} \\
\mathrm{c}=5.1855 \AA^{27}\end{array}$ \\
\hline Nature of Band Gap & Direct & Direct \\
\hline Energy of Band Gap Eg at $300 \mathrm{~K}$ & $3.2 \mathrm{eV}$ & $3.39 \mathrm{eV}$ \\
\hline Exciton Bohr Radius & $\sim 2.5 \mathrm{~nm}^{13}$ & $2.5 \mathrm{~nm}^{13}$ \\
\hline
\end{tabular}




\subsection{Indium Phosphide (InP)}

Indium phosphide is a semiconducting material that has widespread application in today's optoelectronic and telecommunications industries. Indium phosphide has a direct band gap of $1.35 \mathrm{eV}$ at room temperature that is well matched to the solar spectrum and epitaxial indium phosphide is integral to several record efficiency solar cell architectures. ${ }^{28}$

Indium phosphide based devices have been used to produce lasers ${ }^{29}$, photon detectors ${ }^{30}$, optical waveguide switches ${ }^{31}$, and photon filters ${ }^{32}$. Indium phosphide based high electron mobility transistor (HEMT) have also applications in ultra-efficient ultra-linear power amplifiers ideally suited for digital communications systems, satelite networks and highly integrated high speed fiber optic networks. ${ }^{33}$

In addition to that indium phosphide in the form of nanowires is also of significant scientific and technological importance. Potential applications of indium phosphide nanowires include: single phonon detector, fibre optical communications and high speed electronic devices. ${ }^{34,35}$

\section{Crystal Structures and Physical Properties}

Indium phosphide generally adopts the face centred cubic (zinc blende) crystal structure same as that of zinc blende gallium nitride. ${ }^{36}$ However hexagonal wurtzite indium phosphide has also been reported. ${ }^{37}$ The band structure of zinc blende indium phosphide is presented in figure 1.521. Zinc blende indium phosphide is a direct gap semiconductor with a room temperature $\Gamma$ transistion of $1.34 \mathrm{eV}^{38}$ and has an exciton Bohr radius of $\sim 20 \mathrm{~nm}^{14}$. The basic unit cell parameters, band gap and exciton Bohr radius of zinc blende indium phosphide are shown in table 1.522 . 


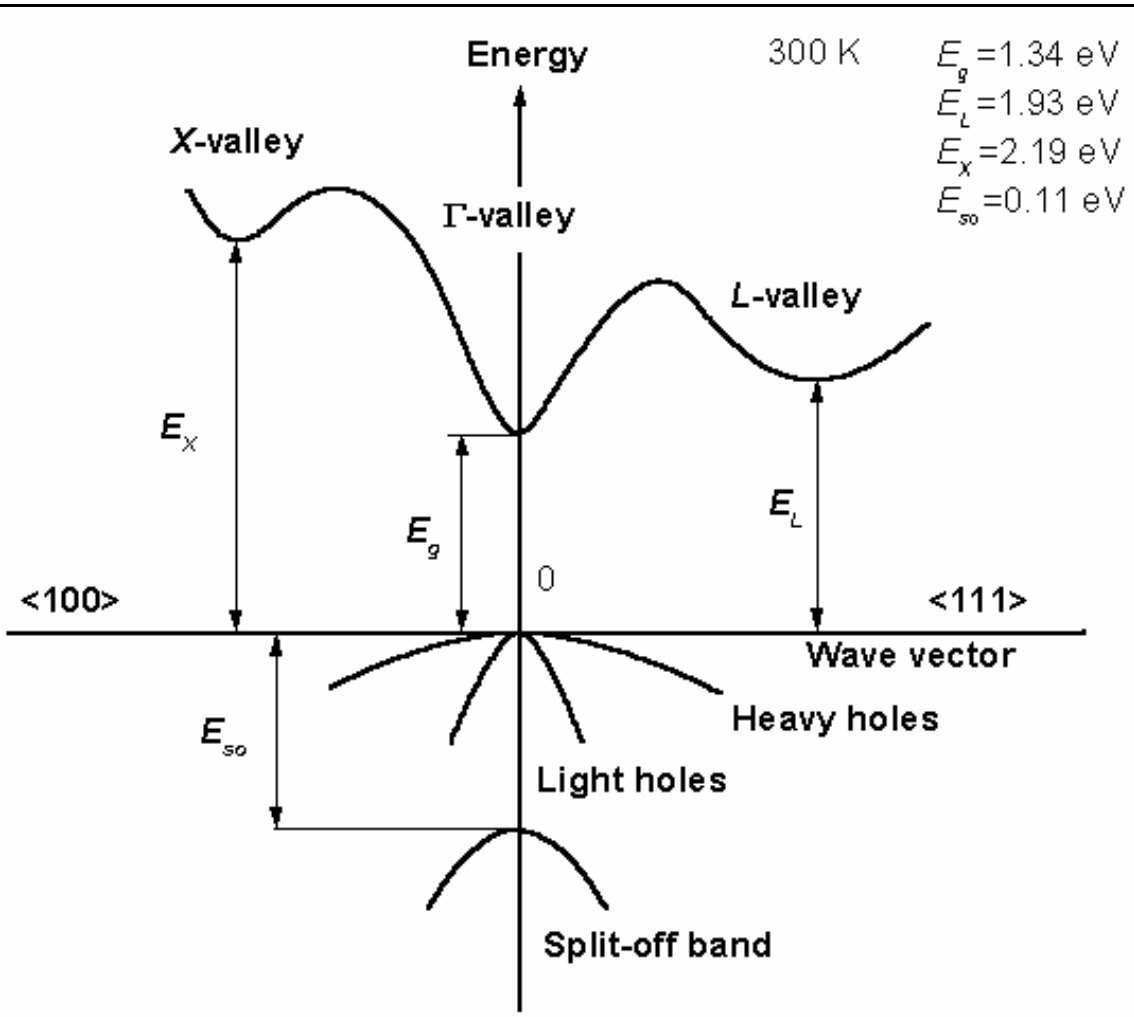

Figure 1.521 Band Structure of face centred cubic (zinc blende) indium phosphide. ${ }^{38}$

Table 1.522 Basic parameters of indium phosphide.

\begin{tabular}{|c|c|}
\hline Structure & Zinc Blende \\
\hline Lattice Parameter at $300 \mathrm{~K}$ & $\mathrm{a}=5.8687 \AA^{36}$ \\
\hline Nature of Band Gap & Direct \\
\hline Energy of Band Gap E $\mathrm{g}_{\mathrm{g}}$ at $300 \mathrm{~K}$ & $1.34 \mathrm{eV}$ \\
\hline Exciton Bohr Radius & $20 \mathrm{~nm}$ \\
\hline
\end{tabular}




\subsection{Zinc Phosphide $\left(\mathrm{Zn}_{3} \mathrm{P}_{2}\right)$}

Zinc phosphide in the form of alpha zinc phosphide $\left(\alpha-\mathrm{Zn}_{3} \mathrm{P}_{2}\right)$ is a semiconductor that has great potential in opto-electronic applications. Proto-type devices based on $\mathrm{Zn}_{3} \mathrm{P}_{2}$ have been fabricated and these include infrared (IR) and UV-sensors, laser and light polarization indicators, solar cells and photon dectectors. ${ }^{40-50}$ Amongst the potential applications, the solar cell devices application is perhaps the most significant. Zinc phosphide is a material of choice for use in solar cell technology for various considerations:

(i) in bulk form, zinc phosphide has a direct band gap quantified to be in between 1.4-1.6 eV, a range ideal for solar energy conversion in photovoltaic devices. ${ }^{41}$

(ii) it has a large optical absorption coefficient and a long minority diffusion length and both contribute to a high current collection efficiency. ${ }^{41,42}$

(iii) both zinc and phosphorus are relative abundant and cheap and would allow the large scale preparation.

(iv) zinc phosphide is thermally stable as may be suggested by its rather high melting point of $1100{ }^{\circ} \mathrm{C}$ under ambient pressure. ${ }^{41}$

\section{Crystal Structures and Physical Properties}

There are two known phosphides of zinc: $\mathrm{Zn}_{3} \mathrm{P}_{2}$ and $\mathrm{ZnP}_{2}$ and both are dimorphic. ${ }^{51}$ The phase diagram of the zinc-phosphorus system is presented as figure 1.530. As may be seen in the phase diagram that both alpha and beta zinc phosphide $\left(\alpha-Z_{3} \mathrm{P}_{2}\right.$ and $\beta$ $\mathrm{Zn}_{3} \mathrm{P}_{2}$ ) only exists above $416{ }^{\circ} \mathrm{C}$. Alpha zinc phosphide transforms into beta zinc phosphide above $850{ }^{\circ} \mathrm{C}$. 


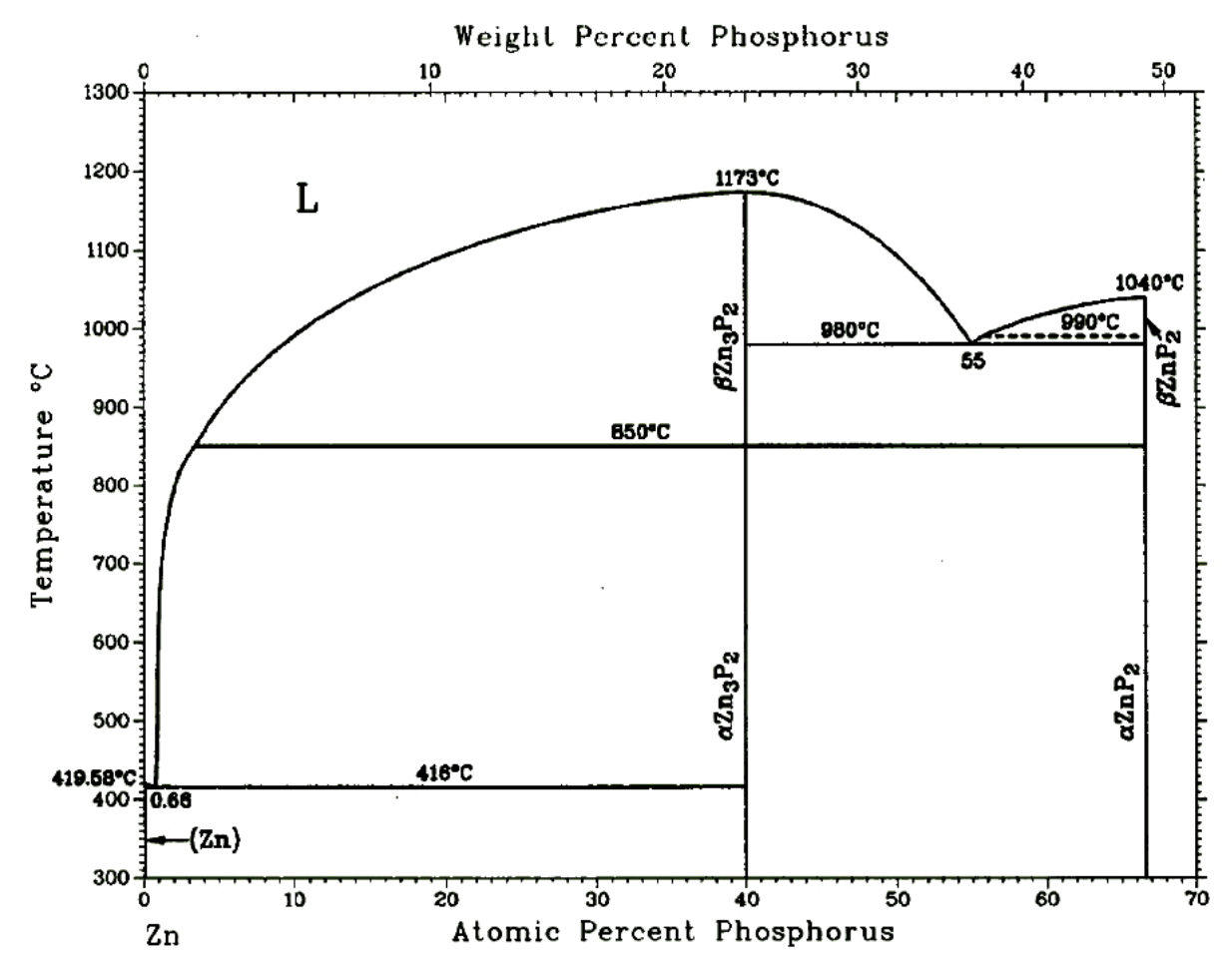

Figure 1.530 The phase diagram of the Zn-P system. ${ }^{49}$

Misiewicz ${ }^{52}$ reported that the crystal structure of $\alpha-\mathrm{Zn}_{3} \mathrm{P}_{2}$ is best represented as a "metal-deficient" antifluoride as shown in figure 1.531. In an antifluoride structure, anions organize in a cubic close-packed fashion and cations occupy all eight tetrahedral sites available in a unit cell. In a defective antifluoride model, a quarter of the cations are missing from the unit cell (2 of 8 ) and thus forming two cations vacancies (represented by squares in figure 1.531). These vacancies exist in pairs and their ordering along the $c$-axis made the volume of the $\alpha-Z_{3} \mathrm{P}_{2}$ unit cell four times as large as the volume of the antifluoride unit cell and therefore transforming from a cubic system into a tetragonal system. The tetragonal unit cell consists of eight $\mathrm{Zn}_{3} \mathrm{P}_{2}$ units with a total of fourty atoms. The zinc atoms are located on four equally spaced planes perpendicular to the $c$-axis. The phosphorous atoms are located in planes in between two adjacent zinc planes. Each zinc atom is surrounded by four phosphorous atoms forming a distorted tetrahedron. Each phosphorous is surrounded six zinc atoms forming a irregular octahedron. 


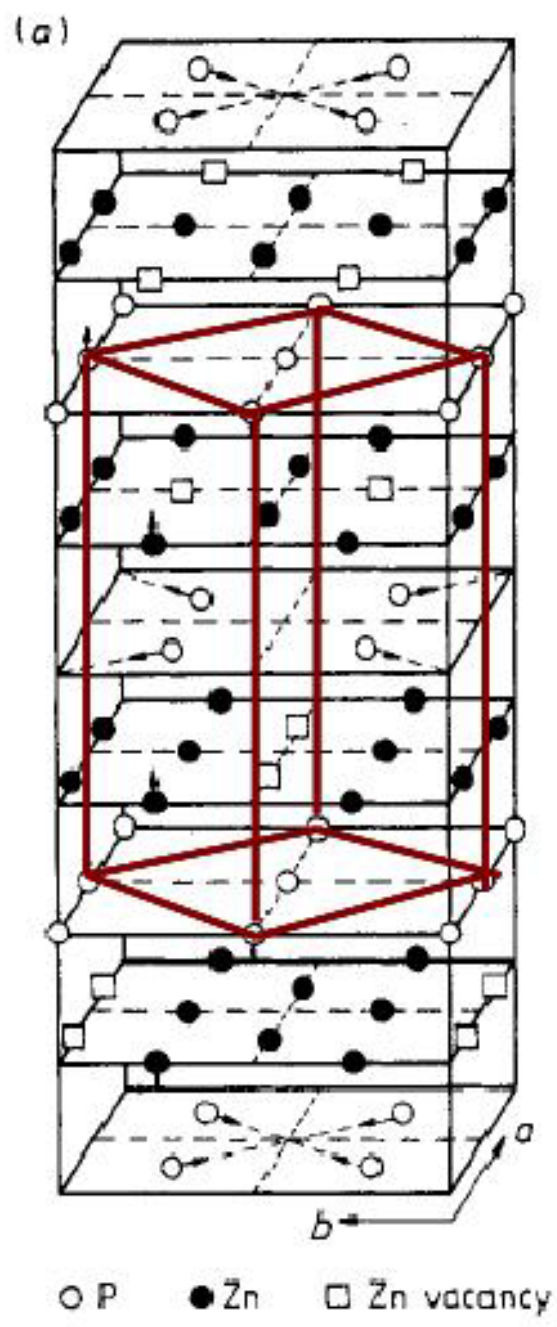

Figure 1.531 Defective antifluoride model of tetragonal $\alpha-\mathrm{Zn}_{3} \mathrm{P}_{2}$ made up of alternating layers of zinc and phosphorous. Arrows depicted the direction of distortion. ${ }^{52}$

Zinc phosphide $\left(\alpha-\mathrm{Zn}_{3} \mathrm{P}_{2}\right)$ has a direct band gap of $1.4-1.6 \mathrm{eV} .^{53}$ Due to its rather complex crystal structure, most calculations presented in literature have been carried out based on simplified crystal structures. ${ }^{53 \mathrm{~b}}$ The band structure of $\alpha-\mathrm{Zn}_{3} \mathrm{P}_{2}$ calculated from its full crystal structure has only been achieved recently by Andrzejewski and Misiewicz and is shown in figure 1.532. ${ }^{53 \mathrm{~b}}$ The Bohr radius of $\alpha-\mathrm{Zn}_{3} \mathrm{P}_{2}$ has not been reported in literature. Nevertheless a blue shift of the band gap has been observed for zinc phosphide nanoparticles less than $3 \mathrm{~nm}$ in size and the shift was attributed to the quantum confinement effect. ${ }^{54}$ The basic unit cell parameters and the band gap of $\alpha$ $\mathrm{Zn}_{3} \mathrm{P}_{2}$ are shown in table 1.533 . 


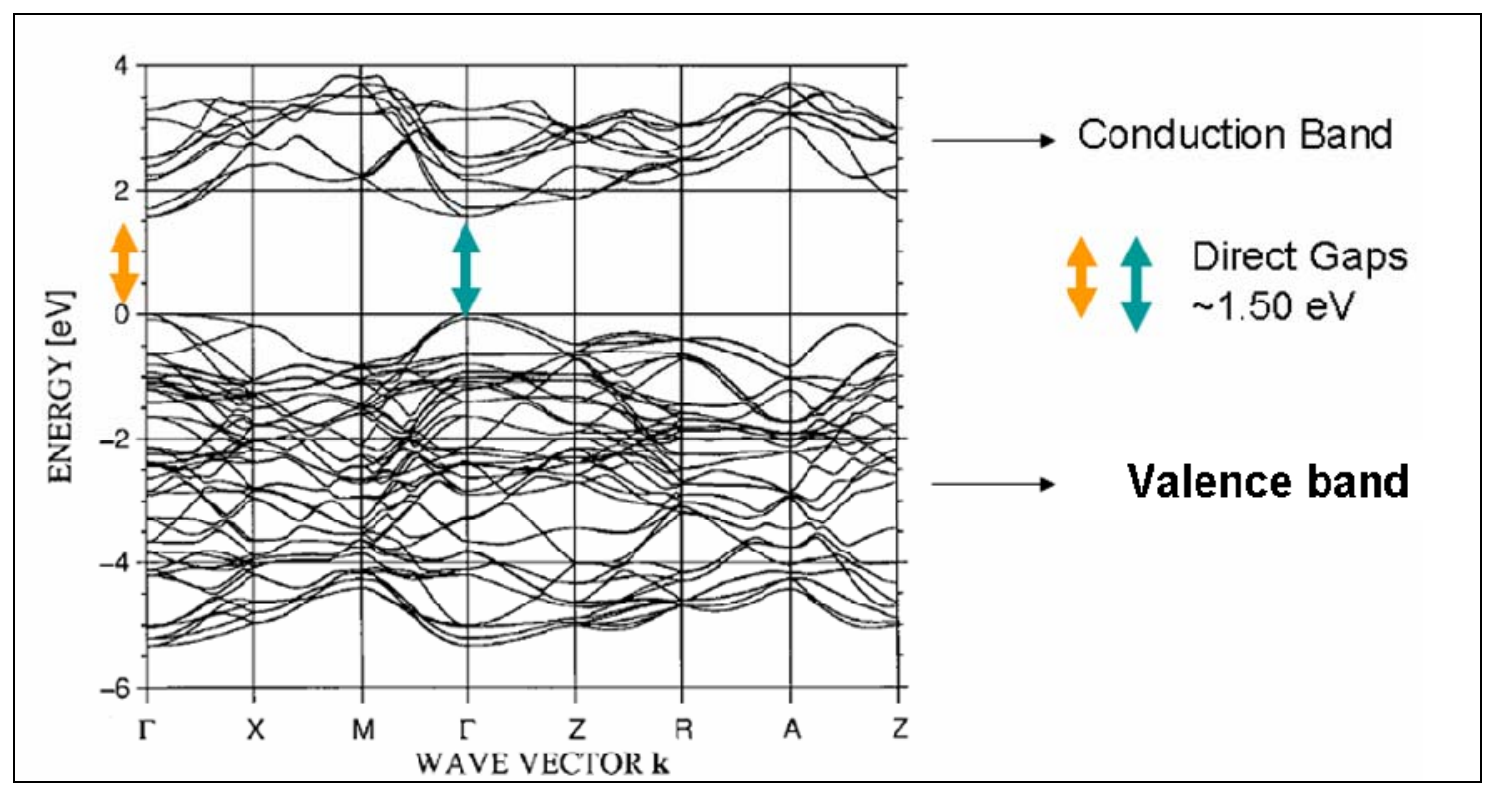

Figure 1.532 Band structure calculated for tetragonal zinc phosphide $\left(\alpha-\mathrm{Zn}_{3} \mathrm{P}_{2}\right)$. Two direct gaps were found both at $\sim 1.5 \mathrm{eV} .{ }^{51 \mathrm{~b}}$

Table 1.533 Basic parameters of zinc phosphide $\left(\alpha-\mathrm{Zn}_{3} \mathrm{P}_{2}\right)$.

\begin{tabular}{|c|c|}
\hline Structure & $\begin{array}{c}\text { Tetragonal } \\
\boldsymbol{\alpha}-\mathbf{Z n}_{3} \mathbf{P}_{2}\end{array}$ \\
\hline Lattice Parameter at $300 \mathrm{~K}$ & $\mathrm{a}=8.0889 \AA^{55}$ \\
$\mathrm{c}=11.4096 \AA$ & 55 \\
\hline Nature of Band Gap & Direct \\
\hline Energy of Band Gap Eg at $300 \mathrm{~K}$ & $1.4-1.6 \mathrm{eV}^{53}$ \\
\hline Exciton Bohr Radius & No literature value available \\
\hline
\end{tabular}




\subsection{Bismuth Metal and Indium Metal}

There are several polymorph of bismuth but there is no general agreement on their structures except for alpha bismuth which is the most stable form at room temperature. ${ }^{56}$ Alpha bismuth has a rhombohedral structure (figure 1.54a) with $\alpha$ and $\beta$ equal to $90^{\circ}$ and $\gamma$ equals to $120{ }^{\circ} \mathrm{C}$ and lattice parameters $a$ and $c$ of $4.5470 \AA$ and $11.8616 \AA$ respectively. ${ }^{57}$ Bismuth metal in the bulk form is brittle, white in colour and has a melting point of $271{ }^{\circ} \mathrm{C}$ under ambient pressure. ${ }^{56}$ Indium metal has a tetragonal crystal structure (figure 1.54b) with $\alpha$ and $\beta$ and $\gamma$ equal to $90^{\circ}$ and lattice parameters $a$ and $c$ of $3.2517 \AA$ and $4.9459 \AA$ respectively. ${ }^{58}$ Indium metal in the bulk form is soft and silvery and it melts at $156{ }^{\circ} \mathrm{C}$ under ambient pressure. ${ }^{56}$
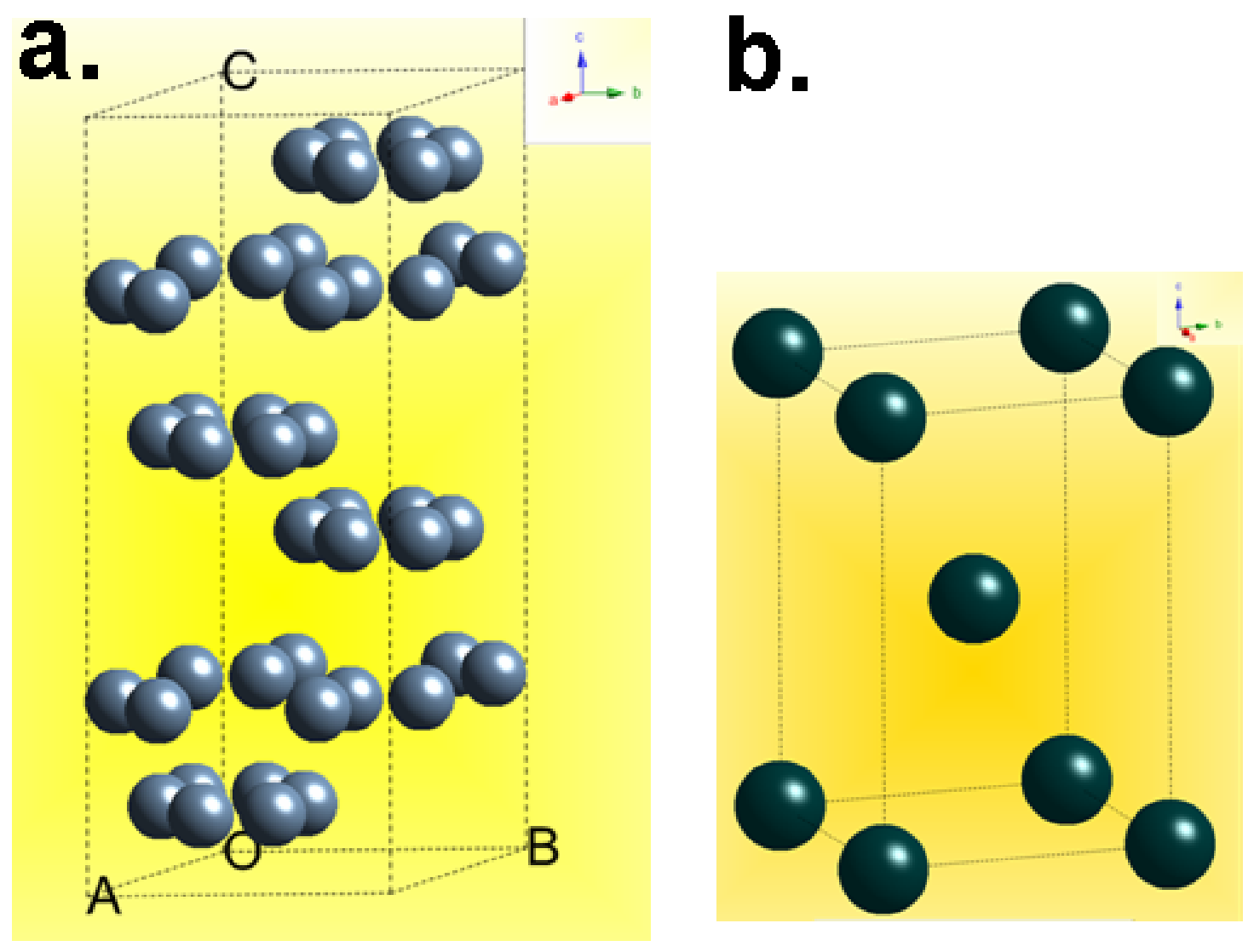

Figure 1.54 (a) A sphere packing model of rhombohedral unit cell of bismuth metal ( $\alpha$ Bi) and (b) A sphere packing model of tetragonal unit cell of indium metal. 


\subsection{References}

1. Schmid,G. Nanoparticles From Theory to Application, 2 Ed., WILEY-VCH Verlag, Weinheim, Germany, 2004.

2. Cushing, B. L.; Kolesnichenko, V. L.; O'Connor, C. J. Chem. Rev. 2004, 104, 3893-3946.

3. Wagner, R. S.; Ellis, W. C. Apply. Phys. Lett. 1964, 5, 89-90.

4. Trentler, T. J.; Hickman, K. M.; Goel, S. C.; Viano, A. M.; Gibbons, P. C.; Buhro, W.E. Science 1995, 270, 1791-1794.

5. Trentler, T. J.; Goel, S. C.; Hickman, K. M.; Viano, A.M.; Chiang, M. Y.; Beatty, A. M.; Gibbons, P. C.; Buhro, W. E. J. Am. Chem. Soc. 1997, 119, 2172-2181.

6. Yu, H.; Li, J.; Loomis, R.; Wang, L. W.; Buhro, W. E. Natur. Mater. 2003, 2, 517-520.

7. Wang, F. D.; Dong, A. G.; Sun, J. W.; Tang, R.; Yu, H.; Buhro, W. E. Inorg. Chem. 2006, 45, 7511-7521.

8. Wang, F. D.; Yu, H.; Li, J. B.; Hang, Q. L.; Zemlyanov, D.; Gibbons, P. C.;

Wang, L. W.; Janes, D. B.; Buhro, W. E. J. Am. Chem. Soc. 2007, 129, 1432714335.

9. Brus L. E. Chem. Phys. 1984, 80, 4403-4409.

10.Alivisatos A. P. Science 1996, 271, 933-937.

11.Alivisatos A. P. J. Phys. Chem. 1996, 100, 13226-13239.

12. Cao, G. Nanostructures and Nanomaterials: Synthesis, Properties and Applications, Imperial College Press, London, United Kingdom, 2004. 
13. Micic. Mic'ic', O. I.; Ahrenkiel, S. P.; Bertram, D.; Nozik, A. J. Appl. Phys. Lett. 1999, $75,478-480$.

14. M. S. Gudiksen, J. F. Wang, C. M. Lieber, J. Phys. Chem. B 2002, 106, 40364039.

15. H. Yu, J. Li, R. Loomis, L. W. Wang, W. E. Buhro, Nat. Mater. 2003, 2, 517 520.

16 Kim, H.; Cho, Y.; Lee, H.; Kim, S.; Ryu, S. R.; Kim, D.; Kang, T.; Chung, K. S. Nano letters 2004, 4, 1059-1062.

17. Nakamura, S.; Pearson, S. J.; Fasol, G. The blue Laser Didoe: The Complete Story, $2^{\text {nd }}$ Ed., Springer-Verlag, New York, United States of America, 2000.

18.(a) http://en.wikipedia.org/wiki/Light-emitting diode.

(b) United States Patent No. 5, 578, 839 (Nakamura et al.).

19. Kocha, S. S.; Peterson, M. W.; Arent, D. J.; Redwing, J. M.; Tischler, M. A.; Turner, J. A. J. Electrochem. Soc. 1995, 142, L238-L240.

20. Pankove, J. I. GaN and Related Materials, Vol. 2, Gordon and Breach Amsterdam, Netherlands, 1997.

21. Qiu, C. H.; Melton, W.; Leksono, M. W.; Pankove, J. I.; Keller, D. P.; DenBaars, S. P. Appl. Phys. Lett., 1996, 69, 1282-1284.

22. Morkoc, H.; Strite, S.; Gao, G. B.; Lin, M. E.; Sverdlov, B.; Burns, M.; J. Apply Phys. 1994, 76, 1363-1398.

23. Ponce, F. A.; Bour; D. P. Nature, 1997, 386, 351-360 
24. Fujii, K.; Karasawa, T.; Ohkawa, K., Japanese Journal of Applied Physics 2005, 44, L543-L545.(b) Waki, I.; Cohen, D.; Lal, R.; Mishra, U.; DenBaars, S. P.; Nakamura, S. Appl. Phys. Lett. 2007, 91, 093519/1-093519/3.

25. (a) http://www.ioffe.rssi.ru/SVA/NSM/Semicond/GaN/index.html

(b) Levinshtein, M.E.; Rumyantsev, S. L.; Shur, M. S.; Properties of Advanced SemiconductorMaterials GaN, AlN, InN, BN, SiC, SiGe, John Wiley \& Sons, New York, United States of America, 2001.

26. Purdy, A. P. Chem. Mater. 1999, 11, 1648-1651.

27. (a) Balkas, C.; Basceri, C.; Davis, R. Powder Diffraction, 1995, 10, 266-268.

(b) JCPDS 00-050-0792.

28. Green, M. A.; Emery, K.; Hisikawa, Y.; Warta, W. Prog. Photovolt: Res. Appl. 2007, 15, 425-430.

29. Moller, L.; Doerr, C. R.; Joyner, C. H.; Zirngibl, M. Electron. Lett. 2000, 36, 540542.

30. Chandrasekhar, S.; Zirngibl, M.; Dentai, A.G.; Joyner, C. H.; Storz, F.; Burrus, C.A.; Lunardi, L. M. IEEE Photonics. Technol. Lett, 1995, 7, 1342-1344.

31. Doerr, C. R.; Joyner, C. H.; Stultz, L. W.; Monnard, R. IEEE Photonics. Technol. Lett. 1998, 10, 117-119.

32. Zirngibl, M.; Joyner, C. H.; Chou, P. C. Electron. Lett. 1995, 31, 1662-1664.

33. Oki, A.K.; Streit, D.C.; Lai, R.; Gutierrez-Aitken, A.; Chen, Y.C.; Grundbacher, R.; Grossman, P.C.; Block, T.; Chin, P.; Barsky, M.; Sawdai, D.; Wojtowicz, M.; Kaneshiro, E.; Yen, H.C.; IEEE Conference Proceedings on Indium Phosphide and Related Materials, 2000. 
34. Duan, X. F.; Huang, Y.; Cui, Y.; Wang, J. F.; Lieber, C. M. Nature 2001, 409, 66-69.

35. Wang, J. F.; Gudiksen, M. S.; Duan, X. F.; Cui, Y.; Lieber, C. M. Science 2001, 293, 1455-1457.

36. (a) Addamiano, A.; J. Am. Chem. Soc. 1960, 82, 1537-1540.

(b) JCPDS 04-004-5372.

37. Mattila, M.; Hakkarainen, T.; Mulot, M.; Lipsanen, H. Nanotechnology 2006, 17, $1580-1583$.

38.(a) http://www.ioffe.rssi.ru/SVA/NSM/Semicond/InP/bandstr.html\#Basic

(b) Levinshtein, M.; Rumyantsev, S.; Shur, M. Handbook Series on Semiconductor Parameters, Vol.1, World Scientific, London, United Kingdom, 1996.

39. Mishra, A.; Titova, L. V.; Hoang, T. B.; Jackson, H. E.; Smith, L. M.; YarrisonRice, J. M.; Kim, Y.; Joyce, H. J. ; Gao, Q.; Tan, H. H.; Jagadish, C. Appl. Phys. Lett. 2007, 91, 263104/1-263104/3.

40. Kakishita, K; Aihara K.; Suda, T. Apply Surf. Sci. 1994, 80, 281-286.

41. Misiewicz, J.; Bryja, L.; Jezierski, K.; Szatkowski, J.; Mirowska, N.; Gumienny, Z.; Placezekpopko, E.; Microelectron. J. 1994, 25, R23-R28.

42. Fagen, E. A. J. Appl Phys. 1979, 50, 6505-6515.

43. Pawlikowski, J. M.; Misiewicz; Mirowska, N. J. Phys. Chem. Solids 1979, 40, 1027-1033.

44. Zdanowicz, W.; Zdanowicz, L. Annu. Rev. Mater. Sci. 1975, 5, 301-328. 
45. Larzarev, V. B.; Schevchenka, V. Ya.; Greenberg, Ya. H.; Sobolein, V. V. II-V Semiconducting Compounds; Nauska: Moscow, Russia, 1978.

46. Bushan, M.; Catalano, A. Appl. Phys. Lett. 1981, 38, 39-41.

47. Pawlikowski, J. M. Infra-Red Phys. 1981, 21, 181-187.

48. Pawlikowski, J. M. Infra-Red Phys. 1988, 28,177-182.

49. Harumi, T.; Zinc Phosphide Solar Cells, Jpn. Kokai Tokyo Koho, 1992. Pattern number JP 04072774.

50. Yang, R.; Chueh, Y.-L.; Morber, J.; Snyder, R.; Chou, L.-J.; Wang, Z. Nano Lett. 2007, 7, 269-275.

51. Schlesinger, M. E.; Chem. Rev. 2002, 102, 4267-4301.

52. Misiewicz, J. J. Phys.: Condensed Matter. 1990, 2, 2053-2072.

53. (a) Yang, R.; Chueh, Y.-L.; Morber, J. R.; Snyder, R.; Chou, L.-J.; Wang, Z. L. Nano Lett. 2007, 7, 269-275. b) Andrzejewski, J.; Misiewicz, J. Phys. Stat. Sol. B 2001, 227, 515-540. (c) Nayak, A.; Rao, D. R. Appl. Phys. Lett. 1993, 63, 592-593.

54. Weller, H.; Fojtik, A.; Henglein, A.; Chem. Phys. Lett. 1985, 117, 485-488.

55. (a) Rao, D.; Nayak, A. J. Mater. Sci. 1992, 27, 4389-4392.

(b) JCPDS 00-04701441.

56. Greenwood, N. N.; Earnshaw, A. Chemistry of the elements ButterworthHeinemann Ltd, Oxford, United Kingdom, 1984. 
57. (a) Swanson, F. Natl. Bur. Stand. (U.S) Circ 539 1954, 111, 12.

(b) JCPDS 00-005-0642.

58. (a) Sailor and G. McCarthy, ICDD Grant-in aid, North Dakota State University, Fargo, North Dakota, USA, 1992. (b) JCPDS 00-044-1246. 


\section{Chapter 2 General Preparative Methodology and Characterization Techniques}

\subsection{Solution Phase Synthesis of Semiconductor and Metal Nanomaterials}

Research in synthesizing nanoparticles in solution has been intense and as a result there exists a great range of methods for solution phase syntheses of nanoparticles. ${ }^{1}$ Amongst the many methods, the method which involves injecting precursors into a hot organic solvent pre-heated to high temperature typically at $200-350{ }^{\circ} \mathrm{C}$ has become popular. ${ }^{2}$ The hot-injection method pioneered by Murray and co-workers produced monodispersed highly crystalline cadmium selenide nanoparticles whose surface chemistry could be altered by ligand exchange chemistry. ${ }^{3}$ The hot injection method offers the ability to temporally separate homogenous nucleation (formed upon hot injection) from crystal growth and annealing. The sparse solubility of inorganic crystals in organic solvents together with the thermal energy available from a hot solvent favours the formation of highly crystalline nanoparticles. The homogeneous nucleation produces monodispersed nuclei from which crystal growth occurs yielding nanoparticles of uniform size and shape.

All but one synthesis reported in this thesis employed organic solvents in which chemical reactions occurred. An attempted synthesis of gallium nitride was conducted in a molten lithium chloride/potassium chloride mixture and details are described in section 3.20 in chapter 3 . All syntheses were carried out under dry oxygen-free nitrogen at ambient pressure. In general, a synthesis was carried out in a three neck flask fitted with a septum, a Liebig condenser and a glass thermal couple housing (figure 2.1). For a hot injection reaction, a precursor solution was injected from a syringe into a pre-heated solution which contained a solvent, a surfactant and sometimes a second precursor. The resulting mixture was further heated for a fixed period of time. 


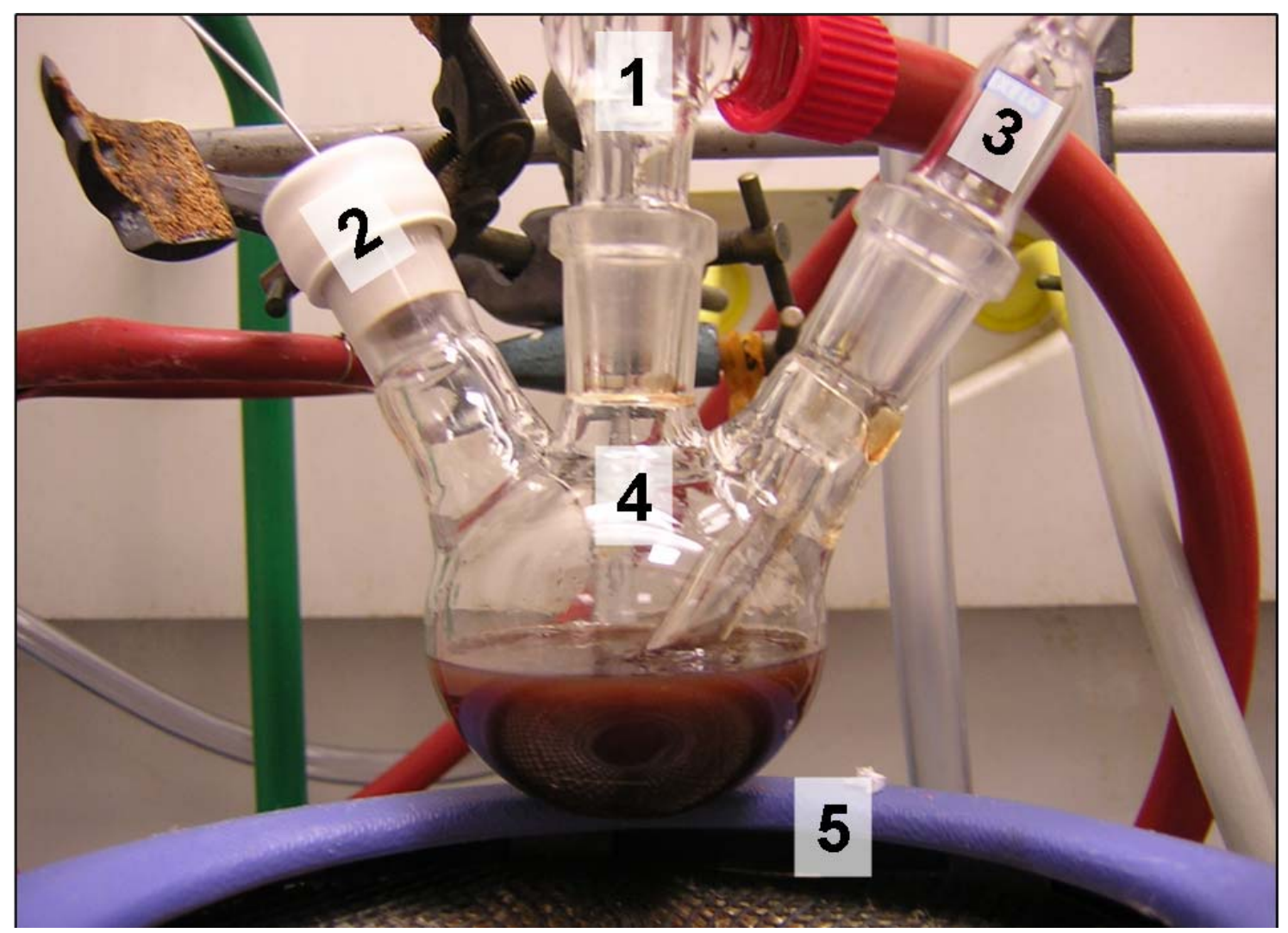

Figure 2.1 A three neck flask system employed. The components include a condenser (1), a septum and a needle (2), a glass thermal couple housing (3), a three neck flask (4) and a heating mantle (5).

The heating mantle provided both stirring and heating simultaneously. The stirring helped better distributing reactants and heat throughout the entire sample and reducing hot spots near the glass surface which was in direct contact with the heating wire. It is important to have the glass thermal couple housing in the solution in order to establish the real temperature of the solution. A temperature gradient of $\sim 10{ }^{\circ} \mathrm{C}$ has been observed when the housing was placed above the solution.

The condenser was connected to a vacuum-nitrogen line. The solvent-surfactant mixture was degassed by heating the solution to reflux in vacuo. The degassing process removed air and any impurities that had boiling points lower than the solvent and surfactant mixture. After the reaction was completed, the solution was cooled by taking the flask away from the heating mantle and placing it on an aluminium mantle (a good heat conductor). The solution stayed under nitrogen throughout the entire course of cooling in order to avoid the product being oxidized. To purify, the mixture was diluted with a certain volume of a solvent such as toluene or chloroform and subjected to 
centrifugation to precipitate a product from the supernatant which contained most of the excess surfactant and impurities. The purified sample was kept as a solution in a glass vial in air. The volume of the solution was usually topped up to approximately the full volume of the glass vial in order to slow down any feasible oxidation processes.

Metal nanoparticles were commonly synthesized via either reductions of metal ions in microemulsions or thermal decompositions of organometallic compounds in the presence of surfactants. ${ }^{1,4}$ The metal nanoparticles prepared in this thesis were of indium and bismuth. To avoid metal particles being oxidized, the reductions were carried out under nitrogen in an organic solvent. The reactions were carried out either at room temperature or at the refluxing temperature of the solvent used. A two neck flask was used instead of a three neck flask and the glass thermal couple housing used in the three neck flask system was dismissed because the temperatures at which reactions occurred were known or fixed. In the growth of semiconductor nanowires the metal nanoparticles in solution were used without further purification.

\subsection{Sample Characterization}

Once a sample had been synthesized, it was important to establish both the structure and the chemical composition. After the identity was established, the size and shape distribution of the sample were investigated before the study of its optical properties. The optical properties of a nanomaterial are highly sensitive to its size, shape, crystal structure, surface chemistry and the presence of defects. ${ }^{5,6}$ In fact, it is the sizedependent properties of nanostructures that have made them scientifically interesting and render them the potentials to be used as fundamental building blocks in new optoelectronic devices. Knowledge of a nanomaterial's structure, size and shape distributions are important information for the interpretation of highly sophisticated optical data.

The crystal structures of the samples produced in this thesis were characterized by analysing their corresponding electron diffraction patterns (ED). The chemical compositions of the samples have been established by energy dispersive X-ray spectrometry (EDS). The size and shape information of the samples were obtained by studying images of the samples produced during transmission electron microscope (TEM) studies. 
High resolution transmission microscope (HRTEM) studies have generated highly detailed structural information about the samples and with careful interpretations crystallographic defects have been detected and characterized. Although TEM/HRTEM have provided useful information such as highly detailed structural information and from EDS local elemental compositions were established, however, only minute portions of the samples were studied in a transmission electron microscope. Care must be taken when generalizing and applying the conclusions to the entire samples.

In this thesis, powder X-ray diffraction (PXRD) was employed whenever the amount of a sample was sufficient. Powder X-ray diffraction analysis provided information such as the crystallographic phase of a sample and whether other phases were present.

Scanning electron microscope (SEM) was also employed in this thesis to study the morphology and sizes of indium phosphide nanowires described in chapter 6 . It allowed the studies on the material's morphology, size and shape distributions on a much larger portion of a material when compared to that used in TEM studies.

In a SEM there is no image forming lens to produce images. Images are formed from secondary electrons or backscattered electrons released from the interaction of an electron beam and a specimen. Images produced from secondary electrons (SEI) are of better resolution than those produced from backscattered electrons and are more sensitive to the surface features of a sample. Images with lower resolution produced from backscattered electrons however contain information about the relative atomic weights of different species. Features that contain heavy elements appear "whiter" than those contain light elements.

The electrons in the electron beam are called primary electrons. Secondary electrons are produced when a primary electron excites an electron in a sample and loses some of its energy in the process. The excited electrons move towards the surface of the sample undergoing collisions until they reaches the surface where they can escape. The mean path length of secondary electrons in many materials is $\sim 1-3 \mathrm{~nm}$. The shallow depth of production of detected secondary electrons makes them very sensitive to topography. 
The shadow produced in an SEM image from secondary electrons is determined by the position of the detector but the view is as if one were looking down the column (parallel to the electron beam's direction).

\subsection{Data Interpretation}

In this thesis, TEM/HRTEM and EDS were employed as principle characterization techniques and most data in the result sections are of TEM/HRTEM images, SAED patterns and EDS spectra. The following section discusses in further details the processes of data interpretation.

\subsection{HRTEM}

Highly detailed images of atomic resolution may be obtained. From the images a crystal in which atoms are highly ordered could be easily distinguished from the carbon background which is amorphous. For example in figure $2.31 \mathrm{a}$, a portion of a crystalline indium phosphide nanowire is illustrated. From the image, a power spectrum (figure 2.31b) was calculated from a Fast Fourier Transform (FFT) of the image. The power spectrum matched the simulated pattern calculated for a face centred cubic indium phosphide looking down the [110] direction. The growth direction of the wire is determined to be [111] by comparing the power spectrum to the simulated diffraction pattern (figure $2.31 \mathrm{~b}$ to figure $2.31 \mathrm{~d}$ ) and the image. A perfect match is when (i) all the inter-planar angles measured between spots in the power spectrum and those measured in the simulated diffraction pattern are the same and (ii) the values of atomic plane spacing (d-spacings) matches that of a reference. The spacing between two planes of atoms may be calculated by direct measurement on the HRTEM image. 


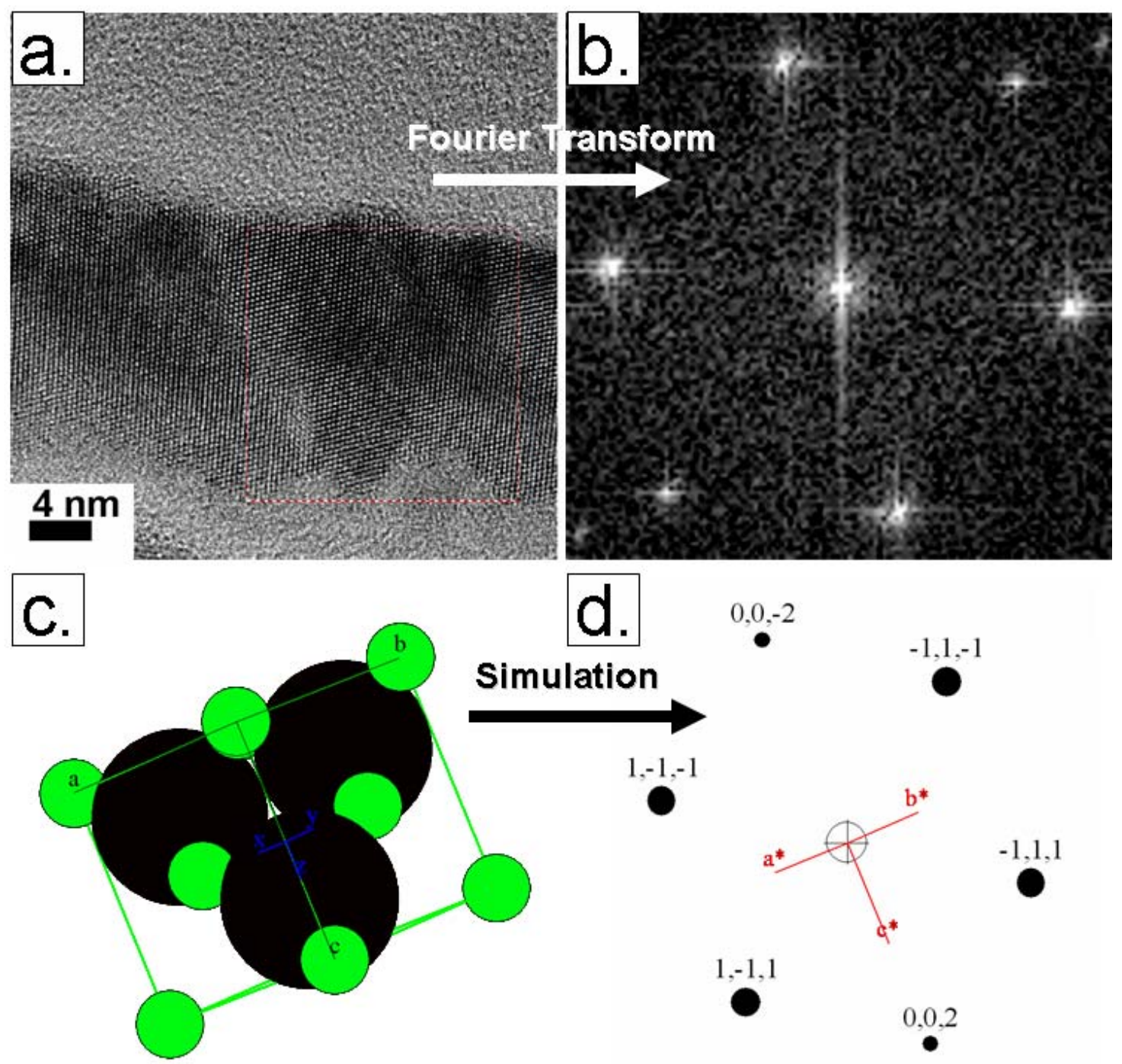

Figure 2.31 (a) HRTEM image of an indium phosphide nanowire; (b) a power spectrum; (c) the unit cell of face centred cubic indium phosphide looking down the [110] direction and (d) the simulated diffraction pattern. 


\subsection{Energy Dispersive X-ray Spectrometry}

Energy dispersive X-ray spectrometry (EDS) allows probing element compositions. Data obtained in EDS is presented as a spectrum. When a single crystal is studied, its chemical composition could be obtained directly from the relative ratios of the elements. For example in figure 2.32 an indium phosphide (InP) crystal with an indium to phosphorous ratio close to $1: 1$ is observed. When a group of many crystals are studied, composition inhomogeneity should be considered when interpreting the corresponding EDS spectrum. Not all crystals may be of the same chemical compositions.

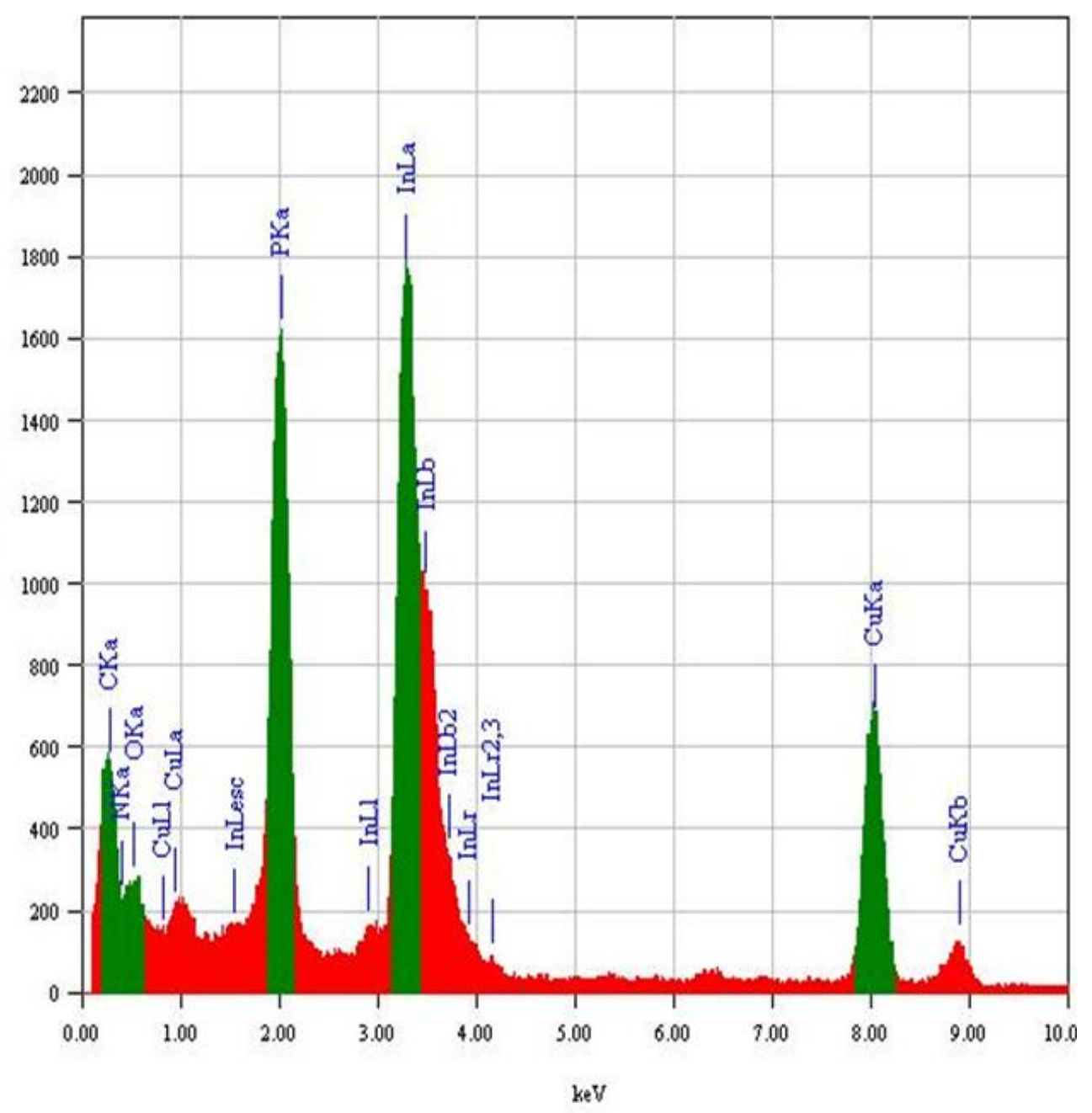

Figure 2.32 An EDS spectrum of indium phosphide shows an indium to phosphorous ratio of close to $1: 1$. 


\subsection{Selected Area Electron Diffraction}

Selected area electron diffraction (SAED) analysis is central to this thesis as it is from the analysis of SAED patterns that the crystal structures of a group of crystals or an individual crystal were elucidated. An immediate use of a SAED pattern is to distinguish a polycrystalline sample from an amorphous or single crystalline sample. If the pattern is of a group of sharp rings the sample is polycrystalline. Taken into consideration the intensities and the d-spacings, atomic planes could be identified and their Miller indexes assigned. From the SAED pattern of a single crystalline sample, the growth direction of an anisotropic nanostructure such as a nanowire could also be determined.

For an unknown compound or a new structure of a known compound the crystal structure and atomic lattice spacings could be determined. To determine a new crystal structure selected area and convergent beam electron diffraction pattern are the proper tools. A sample is tilted and a series of SAED electron diffraction patterns recorded. By analysing the changes observed in the SAED patterns the new structure of a known material or the structure of an unknown material could be established. The actual details are very complicated and there is an excellent account by Bendersky and Gayle. ${ }^{7}$

\subsection{Fundamentals of High Resolution Transmission Electron Microscopy}

High resolution transmission electron microscopy (HRTEM) is a powerful tool that allows direct probing of structural information of crystalline nanostructures. An excellent and detailed account of the technique could be found in the reference by Williams and Carter. ${ }^{8}$

The main principles of an electron microscope are illustrated in figure 2.4. An electron microscope contains an electron source which provides a beam of electrons. Due to the charged nature of electrons this beam can be manipulated and focused by applying magnetic fields to produce diffraction patterns and structural images of materials. 
The electron source used in this research is a lanthanum hexaboride $\left(\mathrm{LaB}_{6}\right)$ single crystal. The electrons are accelerated by an applied potential difference of $200 \mathrm{keV}$. These fast moving electrons have wavelengths $(\lambda=0.0251 \AA)$ much smaller than the inter-atomic distances in a crystal lattice. They are thus diffracted by planes of atoms when interact with a crystal. Due to relativistic effects on the electrons caused by the acceleration by the large potential difference, the precise wavelength of the electrons $\lambda$ is given by

$$
\lambda=\frac{h\left(1-\beta^{2}\right)^{1 / 4}}{\left(2 \mathrm{~m}_{\mathrm{e}} \mathrm{e}_{\mathrm{o}} \mathrm{E}\right)^{1 / 2}}
$$

where $h$ is Plank's constant, $\mathrm{m}_{\mathrm{e}}$ is the mass of an electron, $\mathrm{e}_{\mathrm{o}}$ is the electronic charge, $\mathrm{E}$ is the accelerating voltage and $\beta=\mathrm{V} / \mathrm{C}$ where $\mathrm{V}$ is the velocity of the electron and $\mathrm{C}$ the speed of light in a vacuum.

The electrons are first focused to form a parallel beam by the condenser lenses. This beam strikes the sample. Some of electrons will be diffracted by the sample imparting structural information to the beam. The diffracted and the un-diffracted or primary beams emerge through the sample and are recombined by the objective lenses. The electron beams wavefront can then be focused to a point which lies on a plane (normal to the beam) which is the back focal plane of the objective lens. This produces an electron diffraction pattern. The beam can then be diverged and recombined to produce an image of the sample. By altering the projector lenses either the image or the electron diffraction pattern can be propagated down the microscope and be recorded on a fluorescent screen, photographic plate or a digital camera.

There are many advantages of the microscope, a key one being that due to the far stronger interaction of electrons compared to X-rays with atoms, it is possible to obtain diffraction patterns from individual sub-micron crystals which is not achievable with XRD. A second advantage is that due to recombination of the diffracted beams by the objective lens during the imaging process the phase information of the beam is retained, unlike the case with X-ray diffraction, and there is no phase problem. However, imperfections of the microscope lead to some limitations on the structural resolution that can be obtained. 
These limiting factors are mainly due to most importantly the objective lens. These are the lenses that recombine the diffracted beams from the sample to form the initial image. Any imperfections in this lens such as two-fold astigmatism and spherical aberration must therefore be considered. The degree and orientation of the astigmatism, caused by the departure of the lens bore from a cylindrical nature, can be corrected by electromagnetic coils within the microscope. In theory these coil should allow the complete correction of the astigmatism and this is not normally a major problem in modern microscopes. However, the actual lens in an electron microscope is the magnetic field within the vacuum between the pole-pieces of the objective lens and the geometry of this field is difficult to control. One consequence of this is that beams which are diffracted through different angles are brought into focus at slightly different planes. This is known as spherical aberration and can be corrected for optical lenses by making them aspherical. This cannot be done for magnetic lenses and such effect can to some extent be countered by increasing the focal length. The beams are therefore brought to the optic axis further along than before. This however resulted in a poorer resolution.

Two other important factors affect the resolution of the microscope. These are chromatic aberration and beam divergence. Chromatic aberration originates from the fact the focal length of the magnetic lens is dependent on the energy of the electrons. The electron beam is not perfectly monochromatic having a spread of energies and consequently wavelengths. The inherent thermal energy spread of electrons emitted from the electron source and fluctuations in the accelerating voltage and the objective lens current would also have contributed to the occurrence of chromatic aberration. Beam divergence results from the filament not being a point source and consequently the incident beam is never exactly parallel. Both chromatic aberration and beam divergence reduce the performance of the microscope by introducing an uncertainty in the phase introduced by the objective lens. 


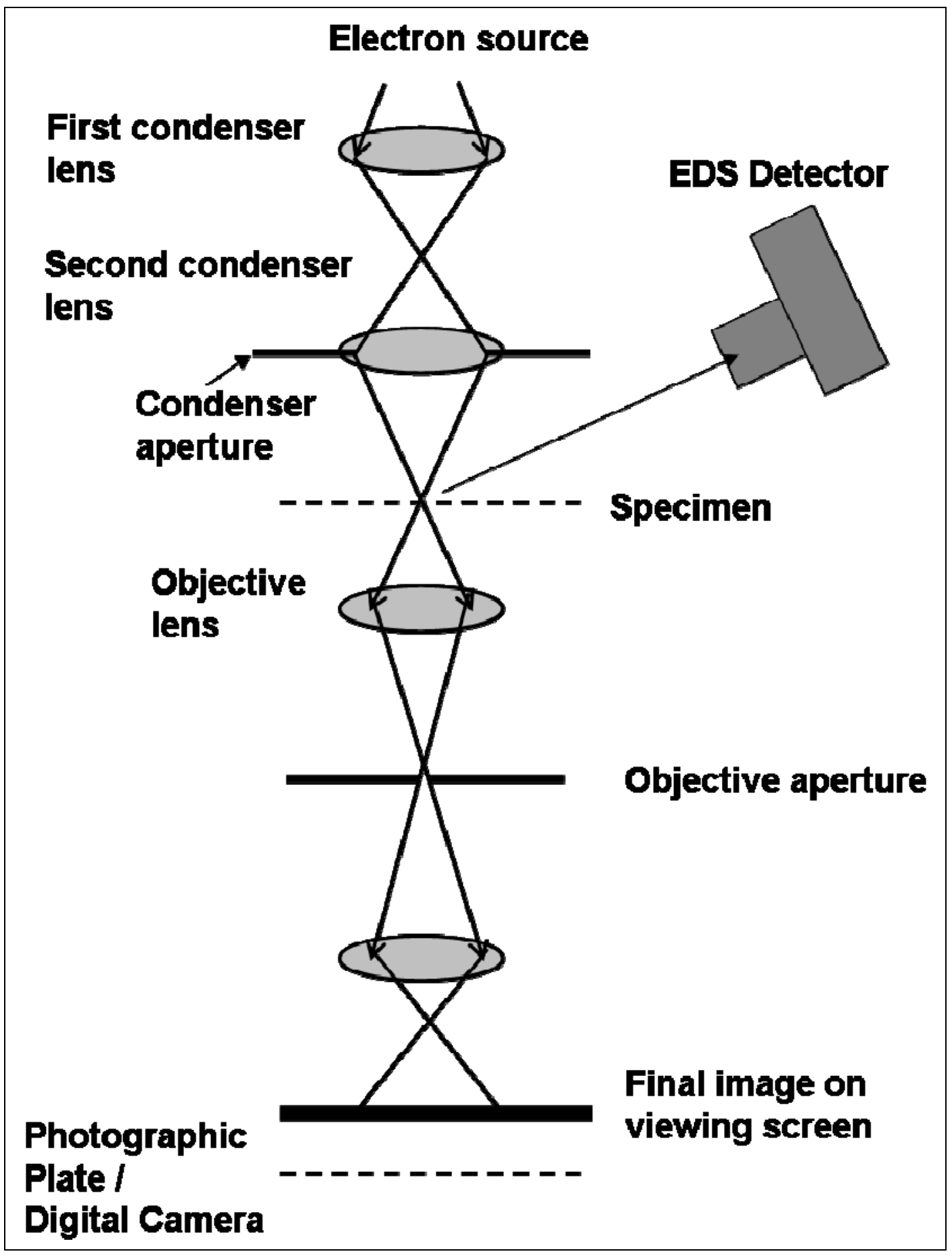

Figure 2.4 A schematic diagram of the structure of a transmission electron microscope. The scheme was constructed based on information available in reference 9 . 


\subsection{Fundamentals of Energy Dispersive X-Ray Spectrometry}

Energy dispersive X-ray spectrometry (EDS) is a useful analytical technique that provides information about the elemental ratios of a material. The beauty of EDS technique lies at its capability of analyzing individual crystals and thus allows the detection of impurities that may be too low in concentration to be detected by powder X-ray diffraction. With modern computer and software quantitative analysis could be derived directly providing a fast and accurate method for sample characterization. However an EDS spectrometer must be standardized accurately before such application.

\section{Generation of X-Rays}

A specimen being bombarded by a focused beam of high energy electrons in a TEM emits X-rays as a result of inelastic scattering of electrons by the specimen. An inelastically scattered electron is lower in energy when compared to the incident electron. Electrons generated at $200 \mathrm{KeV}$ contain sufficient energy to ionize atoms by removing an electron from one of the inner shells before journeying on with reduced energy. An electron from an outer shell would then fall into the inner shell to fill in the vacancy thus stabilizing the atom. As the electron drops from a higher energy shell (outer shell) to a lower energy shell (inner shell) energy must be lost in some form. The energy can be lost either as the emission of a second electron (an Auger electron) or an $\mathrm{X}$-ray photon. The energy of the Auger electron or the X-ray photon is characteristic of an element and can therefore be used as a fingerprint to indicate the presence of a specific element within a crystal. In EDS the X-ray photons are detected. The energies of the emitted X-ray photons are characterized according to the shells involved. Figure 2.50 shows the complete range of X-rays possibly be generated during an EDS analysis. For examples, the $\mathrm{L}$ to $\mathrm{K}$ transition gives $\mathrm{K}_{\alpha} \mathrm{X}$-ray photon and the $\mathrm{M}$ to $\mathrm{K}$ transition gives $\mathrm{K}_{\beta} \mathrm{X}$-ray photon. In a real experiment, all the X-rays may be generated but not all can be detected. The identification of an element is carried out by recognizing a set of X-rays photons emitted which are "fingerprints" of an element. 
The intensity of an X-ray emission may be reduced due to photons being absorbed by the specimen and/or the metal component of the grid on which the crystals sit. Thin crystals less than $30 \mathrm{~nm}$ are considered better candidates for EDS analysis. The absorption by the metal component of the grid can be easily avoided by studying crystals in the centre of the grid away from the metallic grid bars.

The X-ray detector designed for EDS analysis contains a piece of lithium doped silicon crystal. When an X-ray photon is absorbed by the silicon an electron hole pair is produced and therefore a current is induced. The number of electrons generated and therefore the current induced is proportional to the energy of the X-ray photon. The Xray detector can only process a certain amount of X-ray photons in a given time and while one detection is being processed another cannot be acquired. The time delay problem is more severe with pulses of higher energy for if there is too much X-ray photons input the detector will tend to detect the lower energy emissions in preference to the higher energy emissions and this would lead to misleading data. This problem can be overcome by studying only thin crystals and by reducing beam current. 


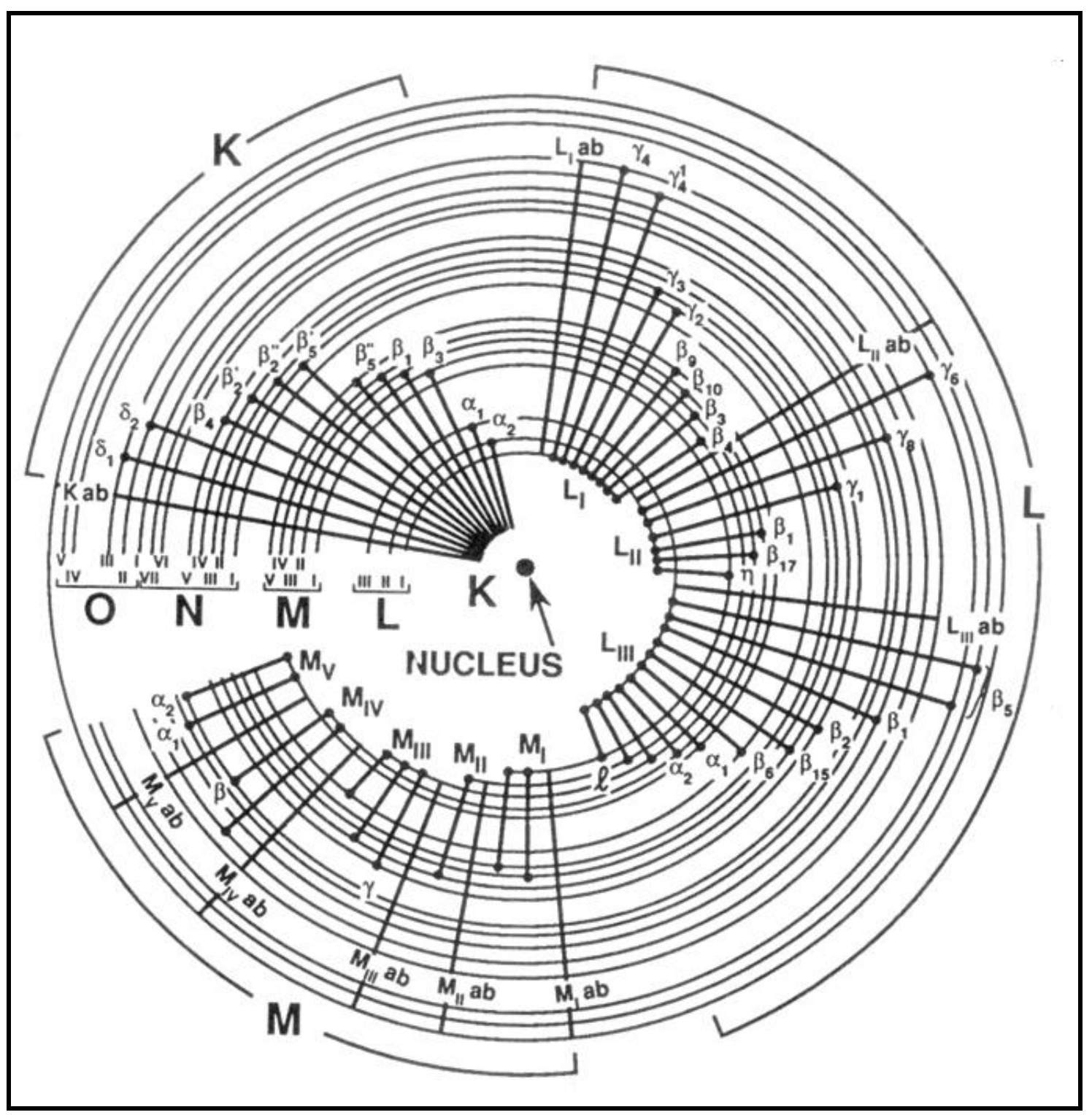

Figure 2.50 The complete range of possible electron transitions that give rise to $\mathrm{K}, \mathrm{L}$, M characteristic X-rays. Not all X-rays are detectable by EDS in the TEM. (Courtesy David B. Williams and C. Barry ${ }^{8}$ ) 


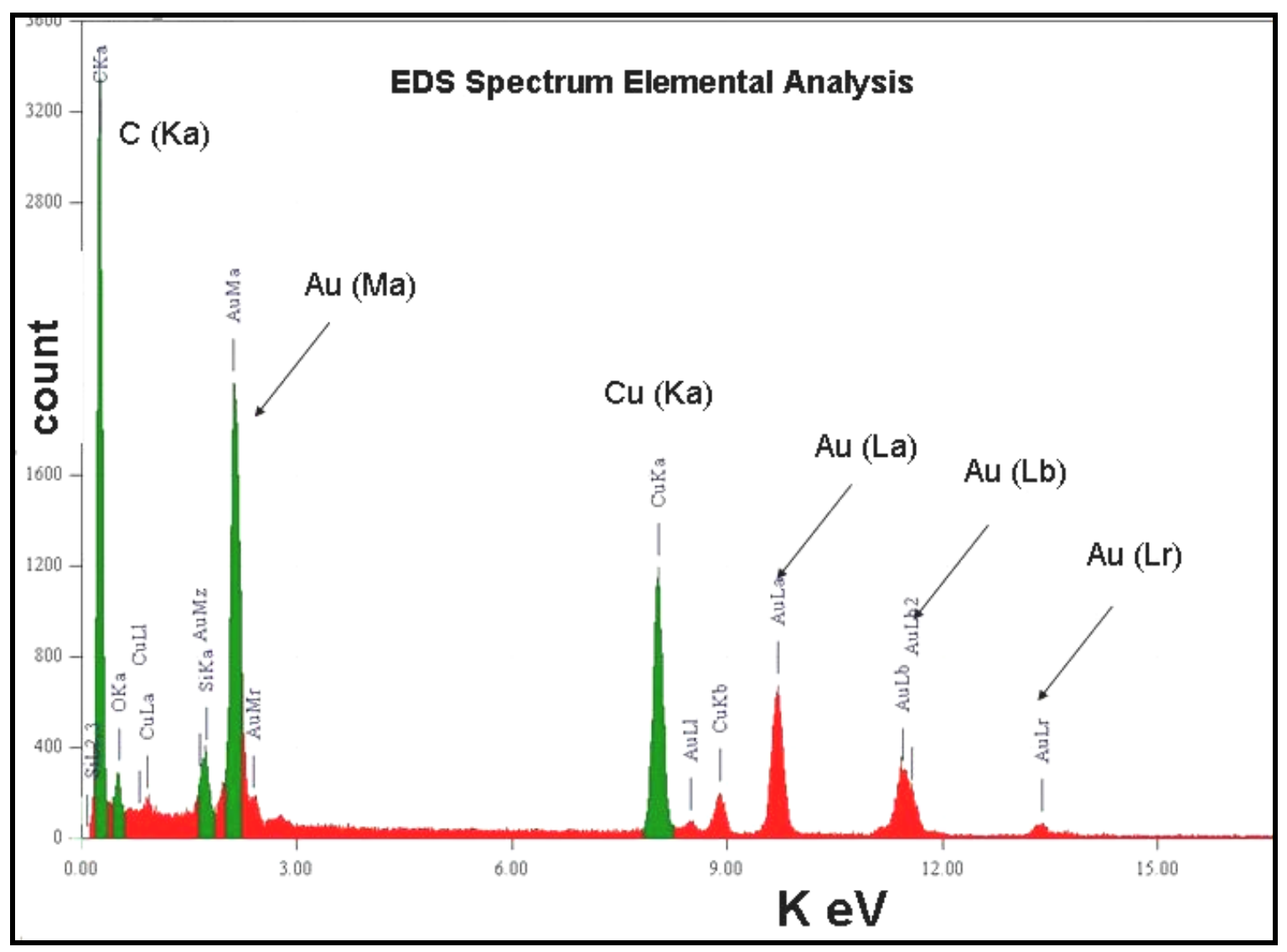

Figure 2.51 An EDS spectrum of gold nanoparticles on a carbon coated copper grid. Heavy elements such as gold and copper give a set of X-rays emission while light element like carbon gives only one $\mathrm{X}$ ray emission.

An EDS spectrum of a sample of gold nanoparticles is presented as figure 2.51. The intensity in the unit of count over a certain period of time is plotted as a function of the energy of X-ray emitted in the unit of electronvolts $(\mathrm{eV})$. With a conventional carbon coated copper TEM grid, X-ray emissions from carbon and copper could be easily observed. A heavy element such as gold would give a higher number of X-ray emissions (from 4-5 different transitions). 


\subsection{Practical Considerations}

\section{Powder X-ray Diffraction}

A Philips X-ray diffractometer with a copper $\mathrm{K}_{\alpha}(\lambda=1.5405 \AA)$ X-ray source was used for phase identification. X-rays diffracted by the sample were collected and processed by the X'pert Data Collector software. The geometry is such that the detector is rotating to collect diffracted X-rays at a particular range of $2 \theta$. The range of $2 \theta$ measured in general ranged between $20-80^{\circ}$. A tension of $40 \mathrm{KV}$ and a current of $20 \mathrm{~mA}$ were used for X-ray diffraction measurements. Samples were prepared by drying a nanoparticle solution on a piece of silicon substrate or directly on a sample holder depending on whether the solvent is capable of dissolving the sample holder.

The instrument was calibrated with polycrystalline silicon to an uncertainty of $\pm 0.03^{\circ}$ in 20. The phase identification was carried out using X'Pert HighScore software by comparing experimental diffraction patterns to those included in the International Centre for Diffraction Data (ICDD) database. Once a match was found by HighScore the match was checked by comparing the experimental diffraction data manually with a literature diffraction data retrieved from the ICDD data base separately using the program ICDD View +-PDF-4+2006. The manual check served to corroborate the match because the parameters HighScore used for pattern matching are unclear. 


\section{FESEM}

A field emission scanning electron microscope JEOL 6700F FESEM, operating at 10 $\mathrm{keV}$, was used for SEM characterizations. The FESEM is equipped with an energy dispersive X-ray analysis unit (EDS). Samples were dried on a piece of silicon substrate. No coating was applied on the samples if they were moderately good electronic conductors.

\section{TEM/ HRTEM}

A JEOL 2011 HRTEM operating at $200 \mathrm{keV}$ was used for TEM and HRTEM analyses. Samples were prepared by drying drops of solutions containing the nanostructures on TEM grid (strong carbon films on 200 mesh copper) purchased from ProSciTech GSCu200C. For single crystal analysis, a double tilt specimen holder was used. The software Gatan DigitalMicrograph was used (i) to detect and correct objective stigmatism in the TEM and (ii) to capture and process images and (iii) to perform Fast Four Transform (FFT) of a high resolution image to produce a power spectrum. An EDS unit was integrated into the electron microscope. The software JEOL Analysis Station was used to process X-ray signals and to identify elements present in the sample. The software CaRine 2.0 was used to simulate diffraction patterns and crystal structures.

\section{Photoluminescence (PL) and Raman spectroscopy}

A solid film prepared by drying a solution containing target nanostructures was used for photoluminescence and Raman studies at $300 \mathrm{~K}$. An $\mathrm{Ar}^{+}$ion laser with a wavelength of $514 \mathrm{~nm}$ was used as the excitation source. The studies were performed using a LabRam Jobin Yvon confocal spectrometer attached to a BX1 Olympus microscope and a $\times 100$ objective lens. The excitation light was reflected on a dichroic mirror and focused by the objective lens onto the surface of the sample film. Signals were collected by the same objective lens, passed through two long wave pass filters, focused onto the entrance slit of the spectrometer with a 600 grating and finally dispersed onto a liquid nitrogen cooled charge coupled device. 


\section{UV-VIS Absorption}

Diluted solutions of the samples in organic solvent were used for UV-VIS absorption measurement. The instrument used was a CARY 100Scan UV-Visible spectrophotometer operating in a double beam mode. The measurements were carried out with a data interval of $1 \mathrm{~nm}$ and a scan rate of $200 \mathrm{~nm}$ per minutes. The measured range spanned from $300-800 \mathrm{~nm}$.

\section{${ }^{1} \mathrm{H}$ and ${ }^{13} \mathrm{C}$ NMR}

NMR spectra were recorded on a Varian Unity Inova 500 operating at $500 \mathrm{MHz}$ for ${ }^{1} \mathrm{H}$ and $125 \mathrm{MHz}$ for ${ }^{13} \mathrm{C}$ at room temperature. The solvent used was $d_{6}$-benzene. 


\subsection{References}

1. Cushing, B. L., Kolesnichenko, V. L., O’Connor, C. J. Chem. Rev. 2004, 104, 3893-3946.

2. Pellegrino, T.; Kudera, S.; Liedl, T.; Javier, A. M.; Manna, L.; Parak, W. J. Small 2005, 1, 48-63.

3. Murray, C. B.; Norris, D. J.; Bawendi, M. G. J. Am. Chem. Soc. 1993, 115, 87068715.

4. Wilcoxon, J. P.; Abrams, B. L. Chem. Soc. Rev. 2006, 35, 1162-1194.

5. Alivisatos, A. P. Science 1996, 271, 933-937.

6. Schmid,G.; Ed. Nanoparticles From Theory to Application; WILEY-VCH Verlag, Weinheim, Germany, 2004.

7. Bendersky, L. A.; Gayle, F. W. J. Res. Natl. Stand. Technl. 2001, 106, 997-1012.

8. Williams, D. B. and Carter, C. B. Transmission Electron Microscopy Basics 1, Plenum Press, New York, United States of America, 1996.

9. (a) Tilley, R. D. PhD Thesis, Department of Chemistry, Cambridge University, 2001.

(b) http://www.uga.edu/ caur/teaching.htm 


\section{Chapter 3 Attempted Syntheses of Crystalline Gallium Nitride Nanoparticles}

\section{Introduction}

This chapter describes research work on the attempted syntheses of gallium nitride nanoparticles. The syntheses were based on two synthetic routes: (i) the metathesis between gallium trichloride and lithium nitride and (ii) the thermal decomposition of an organometallic precursor which contains both gallium and nitrogen.

Conventionally gallium nitride is prepared as solid thin films via either metal organic chemical vapour deposition (MOCVD) ${ }^{1}$ or molecular beam epitaxy (MBE) ${ }^{2}$ methods. MOCVD preparation of gallium nitride in general requires a high reaction temperature $\left(\geq 1000{ }^{\circ} \mathrm{C}\right)$ and MBE preparation an ultra high vacuum (pressure measured in between $10^{-7}-10^{-9} \mathrm{~Pa}$ ). Much effort has been directed towards the syntheses of crystalline gallium nitride nanoparticles at low reaction temperatures $\left(\leq 600{ }^{\circ} \mathrm{C}\right) \cdot{ }^{3-10}$ Low temperature syntheses of gallium nitride have been shown to be achievable and several of the methods employed were listed in table 3.1.

Table 3.1 A list of low temperature syntheses of gallium nitride nanoparticles.

\begin{tabular}{|c|c|c|c|c|}
\hline \multirow[t]{2}{*}{ Precursor } & \multicolumn{2}{|c|}{ Products } & \multirow[t]{2}{*}{ Category } & \multirow[t]{2}{*}{ Reference } \\
\hline & Physical Form & Size (nm) & & \\
\hline$\left\{\mathrm{Ga}\left(\mathrm{N}_{3}\right)\right\}_{\mathrm{n}}$ & Powder & 50 & 1 & 3 \\
\hline$\left[\mathrm{HClGaN}_{3}\right]_{4}$ & Powder & - & 1 & 4 \\
\hline$\left(\mathrm{Et}_{3} \mathrm{~N}\right) \mathrm{Ga}\left(\mathrm{N}_{3}\right)_{3}$ & Colloidal & - & 1 & 5 \\
\hline$\left\{\mathrm{Ga}(\mathrm{NH})_{3 / 2}\right\}_{\mathrm{n}}$ & Powder $^{6} /$ Colloid $^{7}$ & $\sim 3$ & 2 & 6,7 \\
\hline$\left[(\mathrm{Me})_{2} \mathrm{GaN}\left(\mathrm{C}_{6} \mathrm{H}_{6}\right)_{2}\right]$ & Powder & 23 & 3 & 8 \\
\hline $\mathrm{Ga}_{3}\left[\mathrm{~N}(\mathrm{Me})_{2}\right]_{6}$ & Powder $^{9 \mathrm{a}} /$ Colloid $^{9 \mathrm{~b}}$ & $2-4$ & 3 & 9 \\
\hline $\mathrm{GaCl}_{3}+\mathrm{Li}_{3} \mathrm{~N}$ & Powder ${ }^{10 a} /$ Colloid ${ }^{10 b}$ & $30^{10 \mathrm{a}} / 5^{10 \mathrm{~b}}$ & 4 & 10 \\
\hline $\mathrm{GaCl}_{3}+\mathrm{Li}_{3} \mathrm{~N}$ & - & $\sim 5$ & 4 & 11 \\
\hline
\end{tabular}


The methods listed in table 3.1 could be divided into 4 categories based on their reaction pathways:

(1) The thermal decomposition of a gallium azide compound such as $\mathrm{Ga}\left(\mathrm{N}_{3}\right)_{3}{ }^{3-5}$

(2) The deamination of a polymeric gallium imide precursor such as $\left\{\mathrm{Ga}(\mathrm{NH})_{3 / 2}\right\}_{\mathrm{n}}$ under an ammonia atmosphere 6,7

(3) The thermal decomposition of an organometallic precursor such as $\left[(\mathrm{Me})_{2} \mathrm{GaN}\left(\mathrm{C}_{6} \mathrm{H}_{6}\right)_{2}\right]_{2}{ }^{8}$ and $\mathrm{Ga}_{2}\left(\mathrm{NMe}_{2}\right)_{6}{ }^{9}$

(4) The metathesis between gallium trichloride and lithium nitride in organic Solvents 10,11

Amongst these four categories, the methods under category 3 and 4 are of relevance and interest to this chapter. The method employed in category 1 is least favoured due to the use of highly explosive precursors. In category 2 , the preparation of the polymeric gallium imide precursor may be difficult and the precursor has a poor solubility in organic solvents which may be detrimental to particle size control. ${ }^{9}$

The aim of this research was to explore the possibility of synthesizing colloidal crystalline gallium nitride nanoparticles with new methods and new precursors at reaction temperatures less than $450{ }^{\circ} \mathrm{C}$ under ambient pressure. The sizes of the nanoparticles were targeted to be less than $10 \mathrm{~nm}$. It was anticipated that the nanoparticles, being small and soluble, may find applications in catalysis or biological labelling. It was also interesting to investigate if the nanoparticles would exhibit quantum confinement effect with their sizes being close to the Bohr radius of $2.5 \mathrm{~nm}$. Twelve experiments were selected and the corresponding results were described in sections 3.11-3.32.

\subsection{Metathesis of Gallium Trichloride and Lithium Nitride}

The metathesis reaction of gallium trichloride and lithium nitride is an exothermic "exchange" reaction in which gallium nitride $(\mathrm{GaN})$ and lithium chloride are formed. Gallium nitride nanoparticles have been prepared from the metathesis of gallium nitride 
and lithium nitride in pressurized benzene about a decade ago year in $1996 .{ }^{10 a}$ However the nanoparticles prepared have diameter larger than $10 \mathrm{~nm}$ and were prepared as a insoluble powder. It was not until year 2006 that Pan and coworkers reported the preparation of gallium nitride nanoparticles of $10 \mathrm{~nm}$ in size in solution via the metathesis reaction. ${ }^{11}$ However it was unclear from their report whether the particles were colloidal. $^{11}$

The metathesis reaction when carried out in solid state has been found to produce gallium metal and nitrogen gas instead of gallium nitride at ambient pressure and high reaction temperature. ${ }^{12}$ It was argued that the heat produced from the metathesis reaction was sufficiently high to decompose gallium nitride nanoparticles formed in the reaction. It was hoped that by conducting the metathesis reaction in liquid solvents the heat may be dissipated (solvents as heat sinks) and therefore the decomposition process could be prevented.

The attempted metathesis reactions in organic solvents reported here (experiment 1 to experiment 8) were conducted in year 2005 prior to Pan's report ${ }^{11}$. Experiment 10, in which a molten inorganic salt was used as the solvent, were conducted after Pan's report ${ }^{11}$. The solvents employed in this research included benzene, 2,3,11,12-dibenzo1,4,7,10,13,16-hexaoxacyclooctadeca-2,11-diene (dibenzo-18-Crown-6), trioctylamine, hexadecylamine, dibenzofuran, ammonium chloride and lithium chloride/ potassium chloride eutectic mixture. The solvents were chosen based on their polarities. An increase in solvent polarity was expected to facilitate the metathesis reaction due to an improved solubility of lithium nitride.

\subsection{Reaction of Gallium Trichloride and Lithium Nitride in Benzene (Experiment 1)}

Gallium nitride nanoparticles have been previously prepared by Xie and coworkers from the metathesis of gallium trichloride and lithium nitride in pressurized benzene at $280{ }^{\circ} \mathrm{C} .{ }^{10 \mathrm{a}}$ The nitride nanoparticles prepared were of $\sim 30 \mathrm{~nm}$ in size and were insoluble in common solvents such as water or toluene. In this experiment, the reaction of lithium nitride and gallium trichloride in benzene at room temperature under ambient pressure was investigated. No crystalline gallium nitride was expected to form due to a low 
reaction temperature. It was hoped that an amorphous powder containing gallium and nitrogen may be isolated.

A solution of gallium trichloride in benzene was slowly added to a suspension of lithium nitride in benzene at $0{ }^{\circ} \mathrm{C}$. The mixing process was carried out at $0{ }^{\circ} \mathrm{C}$ due to the exothermic nature of the reaction. The mixture was allowed to warm to room temperature with constant stirring. A small amount of black solid particles were observed 15 hours later. From TEM, the black solid was found to be constituted of aggregated spherical particles. From SAED, the particles were amorphous. From EDS the particles contained gallium and no nitrogen. The particles therefore were speculated to be gallium metal. Bulk gallium metal has a melting point of $30{ }^{\circ} \mathrm{C}$ at ambient pressure therefore gallium metal nanoparticles would be expected to melt under bombardment of high energy electrons during TEM studies.

It was believed that the metathesis reaction did not proceed well therefore only a small amount of product was isolated. A report by Pan and coworkers ${ }^{11}$ in 2006 confirmed that the metathesis of gallium trichloride and lithium nitride in benzene was rather slow and no obvious change was noticeable in 48 hours. This has led to conclude that unreacted gallium trichloride remained in benzene would have been lost in supernatant during purification. Unreacted lithium nitride was expected to have reacted with alcohol used in the purification process forming ammonia and lithium alkoxide.

\subsection{Reaction of Gallium Trichloride and Lithium Nitride in Benzene in the Presence of 12-Crown-4 (1,4,7,10-Tetraoxacyclododecane) (Experiment 2)}

From experiment 1, it was found that gallium trichloride and lithium nitride did not react readily in benzene at room temperature. It was speculated at the time that the sparse solubility of lithium nitride in benzene was the cause for a slow reaction. Therefore the metathesis reaction was carried out in toluene in the presence of a polycyclic ether 1,4,7,10-tetraoxacyclododecane (12-Crown-4). 12-Crown-4 was expected to improve the solubility of lithium nitride in toluene as a result of the selective chelating of 12-Crown-4 toward lithium ions. ${ }^{13}$ Crown ethers are well-known for their abilities to chelate alkaline metal ions therefore the solubility of an ionic 
inorganic compound in an organic solvent could be significantly improved. ${ }^{14}$ It was hoped that with its improved solubility, lithium nitride could react more readily with gallium trichloride to form gallium nitride. It was also proposed that by heating up the reaction mixture to $\sim 100{ }^{\circ} \mathrm{C}$ that the speed and degree of reaction may be enhanced. Therefore toluene was used instead of benzene. At this temperature a powder of amorphous not crystalline gallium nitride was expected.

Gallium trichloride, lithium nitride and 12-Crown-4 were mixed and heated to reflux at $\sim 110{ }^{\circ} \mathrm{C}$ overnight. Due to an experimental accident the mixture was exposed to air while being heated. Most of the toluene had been evaporated and a yellow solid was observed as the major product. The yellow solid was expected to be a mixture of lithium chloride (12-Crown-4 complexed) and amorphous gallium nitride. Due to the exposure to air, the solid was expected to have been oxidized at least partially.

After purification, a white solid precipitate was isolated. The precipitate dissolved in dichloromethane to give a clear solution. From TEM, the white precipitate was constituted of nanoparticles of $\sim 2 \mathrm{~nm}$ in size. However the particles have very low contrast and no TEM image of sufficiently good quality was obtained at the time. The particles were believed to be of amorphous gallium oxide due to their exposure to air. However, due to lack of strong evidences the identity of the nanoparticles could not be concluded with confidence.

It was believed that in the presence of 12-Crown-4 at a reaction temperature of $110{ }^{\circ} \mathrm{C}$ the metathesis reaction had been enhanced, evidenced by the isolation of a yellow solid precipitate. No such precipitate was isolated in experiment 1 when the metathesis was carried out in the absence of 12-Crown-4 at room temperature. The speculation was supported by Pan and coworkers observation in which diethyl ether was found capable of increasing the speed of the metathesis reaction to produce amorphous gallium nitride. They attributed the enhanced reaction rate to the interaction between diethyl ether and lithium ions present in lithium nitride. 


\subsection{Testing Dibenzo-18-crown-6 (2,3,11,12-Dibenzo- 1,4,7,10,13,16-Hexaoxacyclooctadeca-2,11-Diene) as Solvent (Experiment 3)}

It was proposed that 2,3,11,12-dibenzo-1,4,7,10,13,16-hexaoxacyclooctadeca-2,11diene (dibenzo-18-Crown-6) could be used as a replacement for 12-Crown-4. Dibenzo18-Crown-6 is known to chelate potassium and sodium ions well but less to lithium ions due to the cavity of its polycylic ring is significantly larger than the ionic radius of a lithium ion. ${ }^{14}$ Nevertheless a weak interaction between dibenzo-18-Crown-6 and lithium ions was expected. It was also proposed that a reaction temperature of $\sim 300{ }^{\circ} \mathrm{C}$ should be used in order to produce crystalline nanoparticles. Dibenzo-18-Crown-6 has a calculated boiling point of $>350{ }^{\circ} \mathrm{C}$ at ambient pressure. ${ }^{15}$ The boiling point and thermal stability of dibenzo-18-Crown-6 was tested prior being used as a solvent and the details were described in the following paragraph.

A sample of dibenzo-18-Crown-6 was heated under nitrogen to form a clear liquid. The liquid did not reflux at $350{ }^{\circ} \mathrm{C}$ indicating a higher boiling point. Half an hour after being heated at $350{ }^{\circ} \mathrm{C}$, the clear liquid changed into a black viscous liquid. The black liquid was expected to be of polycyclic aromatic hydrocarbons which were commonly observed during incomplete combustions of carbon containing fuels. However no investigation was launched into the nature of the black liquid. No metathesis reaction of gallium trichloride and lithium nitride was subsequently carried out due to thermal instability of dibenzo-18-Crown-6. 


\subsection{Reaction of Gallium Trichloride and Lithium Nitride in Trioctylamine (Experiment 4)}

Trioctylamine is a liquid at room temperature and has a boiling point of $\sim 350{ }^{\circ} \mathrm{C}$ under ambient pressure. Being a tertiary amine it was expected to complex gallium ions. An experiment was carried out heating a mixture of gallium trichloride, lithium nitride and trioctylamine at $\sim 340{ }^{\circ} \mathrm{C}$. A solid precipitate was isolated after purification.
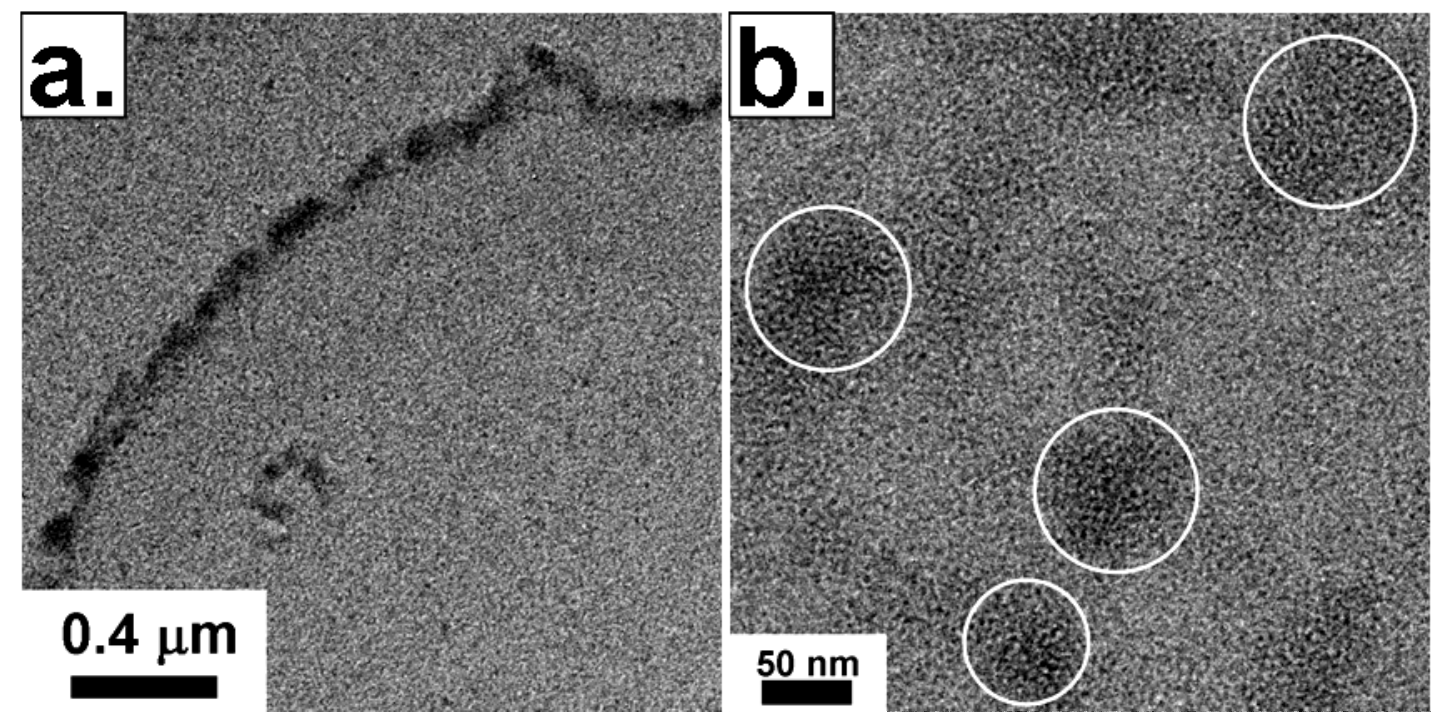

Figure 3.14 Low magnification TEM images of nanoparticles. (a) a chain of groups of nanoparticles and (b) several groups of small particles.

As may be seen in figure 3.14, the solid is constituted of small nanoparticles of $\leq 5 \mathrm{~nm}$. When dried on a TEM grid, the nanoparticles have the propensity to form groups and chains. The contrast of the image has been increased in order to discriminate the nanoparticles from the carbon background. From SAED, the nanoparticles were found to be amorphous. EDS analysis showed that the nanoparticles contained mainly gallium and a small amount of nitrogen. The nitrogen signals may come from a combination of a small amount of nitride particles and trioctylamine which had remained as surfactants. However, it could not be concluded with confidence that the particles were constituted of gallium nitride. Due to the lack of sufficient evidences the particles were believed to be gallium metal nanoparticles. It was postulated that the reaction had proceeded to produce gallium metal and nitrogen gas instead of gallium nitride. The reaction temperature of $340{ }^{\circ} \mathrm{C}$ may be too high for trioctylamine to act as an efficient heat sink. 
It was therefore proposed that a reaction temperature of $\sim 250{ }^{\circ} \mathrm{C}$ should be more appropriate.

\subsection{Reaction of Gallium Trichloride and Lithium Nitride in Hexadecylamine (Experiment 5)}

Hexadecylamine is a primary amine with a boiling point of $330{ }^{\circ} \mathrm{C}$ under ambient pressure. It was expected to serve as an effective heat sink which would dissipate the heat from the metathesis reaction away from nanoparticles during their formation. An experiment was carried out heating a mixture of gallium trichloride, lithium nitride and hexadecylamine to $230{ }^{\circ} \mathrm{C}$ in 20 minutes. A solid precipitate was isolated after purification.

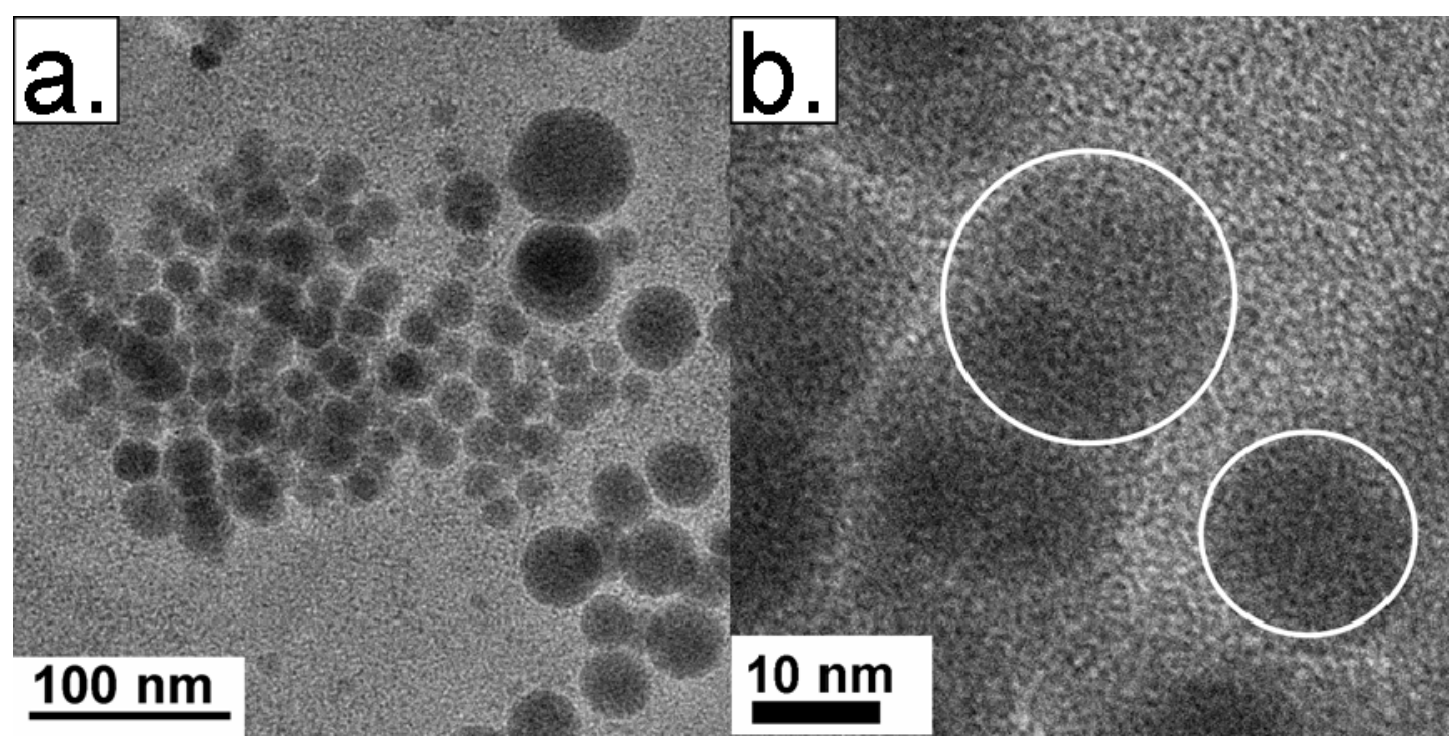

Figure 3.150 (a) TEM images of nanoparticles and (b) a high resolution image showing the amorphous nature of the nanoparticles. 


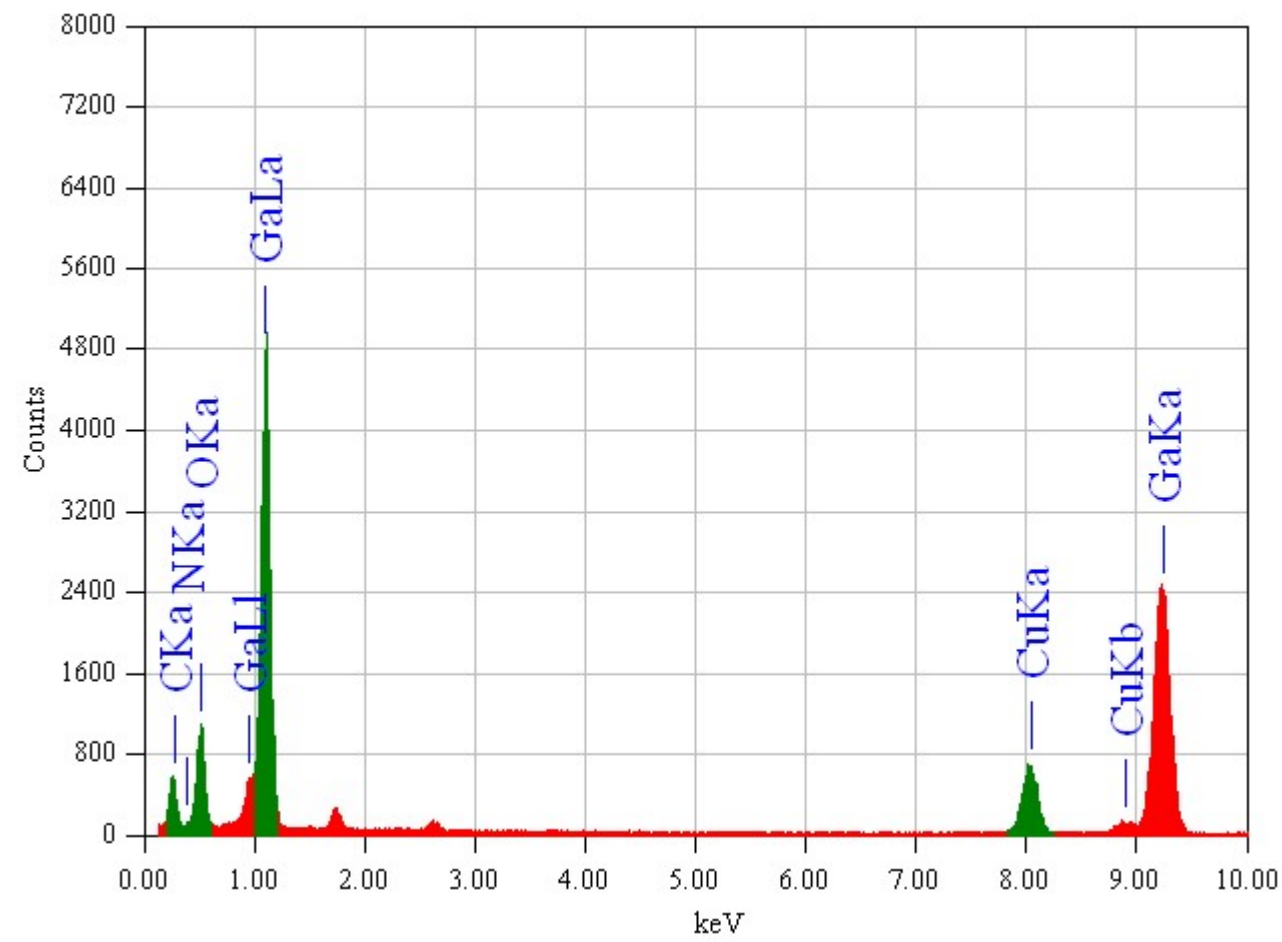

Figure 3.151 An EDS spectrum shows that the nanoparticles contained mainly gallium.

As may be seen in figure 3.150 a, the solid is constituted of segregated spherical nanoparticles $\sim 15 \mathrm{~nm}$ in size. From HRTEM the particles were found to amorphous due to the lack of lattice fringes (figure $3.150 \mathrm{~b}$ ). From SAED, the nanoparticles were confirmed to be amorphous. EDS analysis (figure 3.151) showed that the nanoparticles were composed of mainly gallium. No significant amount of nitrogen was detected. The particles produced in this experiment were believed to be amorphous gallium metal nanoparticles. The formation of gallium metal nanoparticles was attributed to the thermal decomposition of gallium nitride to gallium metal and nitrogen gas. The heat produced from the metathesis reaction was believed to cause the local temperature around nitride particles becoming sufficiently high and as a result of overheating the thermal decomposition process was triggered. 


\subsection{Reaction of Gallium Trichloride and Lithium Nitride in Trioctylamine /Hexadecylamine Mixture (Experiment 6)}

The nanoparticles produced from trioctylamine (experiment 4) were found to contain both gallium and nitrogen and was of $\sim 5 \mathrm{~nm}$ in size. The particles produced from hexadecylamine (experiment 5) contained only gallium and was of $\sim 15 \mathrm{~nm}$ in size. The purpose of this experiment was to investigate if amorphous particles containing both gallium and nitrogen could be formed and if the particle size could be tuned in between 5 to $15 \mathrm{~nm}$ by carrying out the metathesis reaction in a mixture of trioctylamine and hexadecylamine. It was hoped that once produced the particles could be thermal annealed to produce crystalline gallium nitride nanoparticles.

A mixture of gallium trichloride, lithium nitride and trioctylamine was prepared as a light grey viscous solution. The solution was injected into a clear liquid of hexadecylamine at $200{ }^{\circ} \mathrm{C}$. The resulting grey mixture was first heated at $\sim 200{ }^{\circ} \mathrm{C}$ for 5 hours and then $300{ }^{\circ} \mathrm{C}$ for another 5 hours. It was hoped that during the first 5 hours, the metathesis would proceed and that the reaction temperature would not be too high to result in the thermal decomposition of gallium nitride. The heating process at $300{ }^{\circ} \mathrm{C}$ for 5 hours was expected to improve the crystallinity of the nanoparticles.

A wet brown solid was obtained after a total 10 hours of heating. The solid was cooled to $\sim 100{ }^{\circ} \mathrm{C}$ and diluted with ethanol to form a solution. The resulting ethanoic solution appeared green when viewed from the front (transmitted light) and red when viewed from the sides (scattered light). A black solid precipitate was isolated after centrifugation but was lost prior to characterization due to an experimental accident. The ethanoic supernatant remained colourful. After the removal of ethanol, a brown liquid $(<0.2 \mathrm{~mL})$ was obtained. From TEM, the liquid was found to contain no solid particles. From EDS, the liquid contained gallium, carbon and nitrogen. It was believed the solution contained micelles whose sizes were such that red light was strongly scattered. Interestingly a very similar liquid was obtained in an attempt to thermally decompose an organometallic gallium compound in a mixture of trioctylamine and hexadecylamine (experiment 12). No further investigations into the chemical composition of the liquid were carried out. The composition of the species remains unclear. 


\subsection{Reaction of Gallium Trichloride and Lithium Nitride in Dibenzofuran (Experiment 7)}

Chen and Liang reported the synthesis of crystalline gallium nitride nanoparticles from the metathesis of gallium trichloride and lithium nitride in dibenzofuran. ${ }^{10 \mathrm{~d}}$ Dibenzofuran has a polar furan moiety and a boiling point of $\sim 280{ }^{\circ} \mathrm{C}$ at ambient pressure. This experiment aimed at reproducing Chen and Liang's synthesis before making any possible improvement. A mixture of gallium trichloride, lithium nitride and dibenzofuran was heated at $280{ }^{\circ} \mathrm{C}$ for 4 hours to form a brown solution. A dark grey solid was isolated from the brown solution after purification.

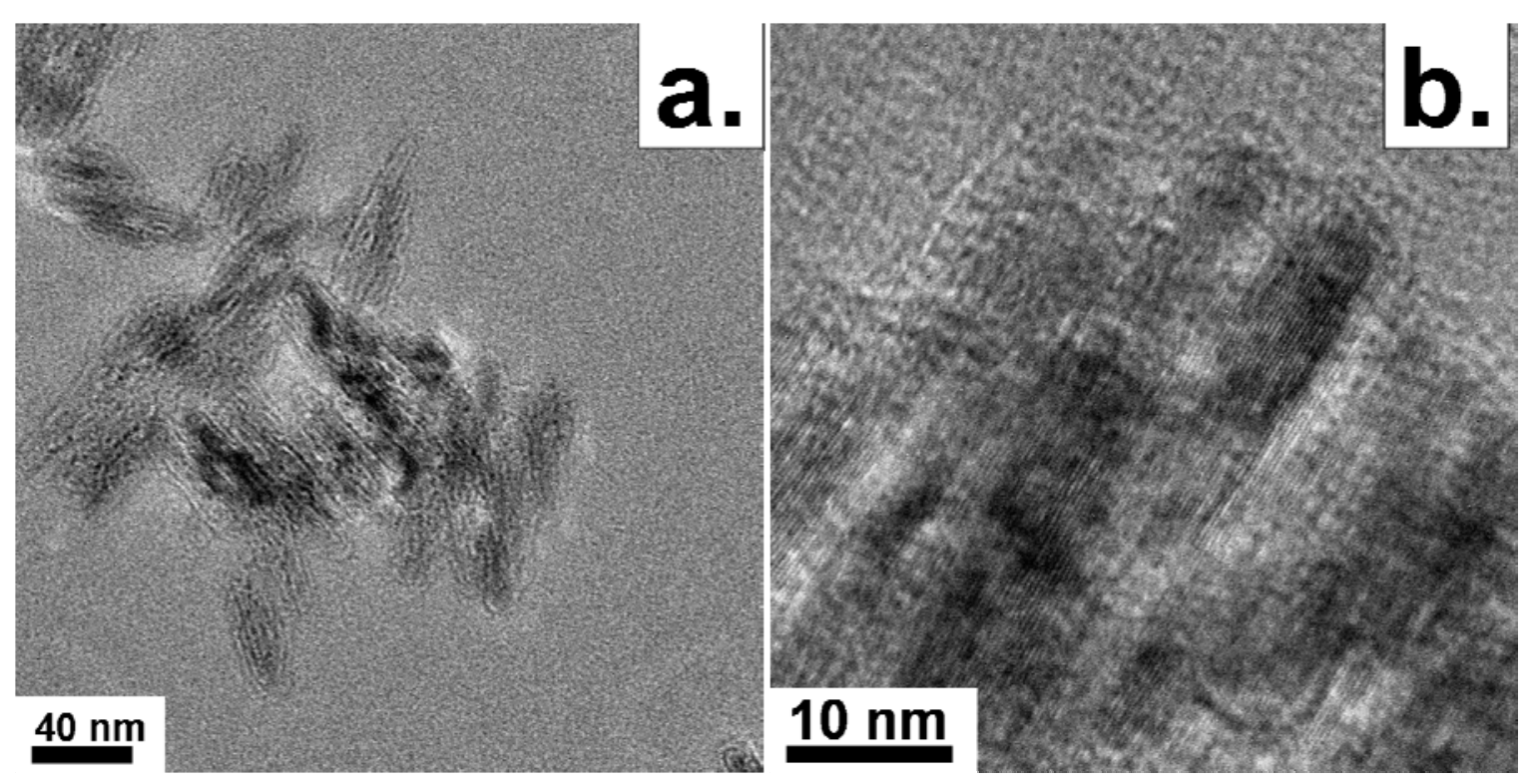

Figure 3.17 TEM images (a) a group of nanorods (b) nanorods with lattices fringes resolved.

From TEM and SAED, the solid was found to be constituted of mainly amorphous nanoparticles in the micrometer range. A small amount of crystalline rod-like nanostructures (figure 3.17a) were also observed. The rod-like nanostructures were highly crystalline and clear lattice fringes may be seen in figure $3.17 \mathrm{~b}$. From EDS, the nanorods were found to be consisted of mainly gallium (55-75\%) and a small amount of oxygen $(\sim 12 \%)$. No nitrogen content was detected. The amorphous particles were expected to be gallium metal and the crystalline rod-like nanostructures gallium oxide. The products isolated in this experiment were significantly different to the $5 \mathrm{~nm}$ crystalline gallium nitride as reported by Chen and Liang ${ }^{10 d}$. It was unclear whether it was a reproducibility issue. 


\subsection{Reaction of Gallium Trichloride and Lithium Nitride in Octadecene/ Dibenzofuran Mixture (Experiment 8)}

From experiment 7, it appeared that the metathesis reaction of gallium trichloride and lithium nitride in dibenzofuran at $280{ }^{\circ} \mathrm{C}$ produced mainly amorphous gallium metal and nitrogen gas instead of gallium nitride. It was suggested that the heat generated from the metathesis reaction must be dissipated effectively away from the nanoparticles in order to favour the formation of gallium nitride nanoparticles. One approach proposed to be taken was to add a diluted solution of gallium trichloride to a mixture of lithium nitride in dibenzofuran at $200{ }^{\circ} \mathrm{C}$. The solvent used to dissolve gallium trichloride must have a high boiling higher than the $200{ }^{\circ} \mathrm{C}$ and the resulting solution should be chemically stable.

Octadecene is a liquid at room temperature and has a boiling point of $\sim 300{ }^{\circ} \mathrm{C}$ under ambient pressure. It has been used commonly to prepare crystalline semiconductor nanoparticles. ${ }^{16}$ It was chosen for this experiment for it was expected to dissolve gallium trichloride readily and it has a boiling point higher than the anticipated reaction temperature of $250{ }^{\circ} \mathrm{C}$. An unexpected green viscous liquid formed from dissolving gallium trichloride in octadecene at room temperature. The chemical composition of the green liquid is unclear but it may contain polymerized species which would explain the increase in viscosity. The metathesis reaction was therefore not being carried out due to the difficulty in transferring the viscous liquid via a syringe or a pipette. The green viscous appearance of the liquid also suggested the gallium precursor has changed to other species.

\subsection{Testing Ammonium Chloride as Solvent}

\section{(Experiment 9)}

In the literature it was reported that iodine when used as a heat sink during the solid state metathesis of gallium trichloride and lithium nitride helped improve the yield of gallium nitride nanoparticles. ${ }^{18}$ It was suggested that the thermal decomposition process of gallium nitride was prevented by having iodine to absorb and dissipate the heat via sublimation. ${ }^{18}$ Ammonium chloride is known to melt and sublime at $\sim 340{ }^{\circ} \mathrm{C}$ under ambient pressure. ${ }^{17}$ It was proposed that ammonium chloride may be used both as a solvent and a heat sink (via sublimation) for the metathesis reaction of gallium 
trichloride and lithium nitride. In addition to that molten ammonium chloride was expected to be an ideal solvent for the dissolution of lithium nitride. In this experiment, ammonium chloride was heated to $\sim 340{ }^{\circ} \mathrm{C}$ under nitrogen at ambient pressure. It was found to have partially sublimed and no liquid state was observed. Therefore the metathesis reaction was not carried out due to the lack of liquid state. It is however anticipated in a pressurized vessel the system may work without the problem of sublimation. A liquid state is required to dissolve lithium nitride and gallium trichloride and to ensure uniform reaction temperature and precursor concentrations.

\subsection{Reaction of Gallium Trichloride and Lithium Nitride in Molten Lithium Chloride/Potassium Chloride Eutectic Mixture (Experiments 10)}

Molten lithium chloride / potassium chloride mixture was proposed as a solvent for the metathesis of gallium trichloride and lithium nitride because it has a eutectic temperature of $352{ }^{\circ} \mathrm{C}$ at the correct mole ratio and it would not sublime at the melting temperature. ${ }^{19}$ The molten mixture has been found capable of dissolving uranium nitride in the literature. ${ }^{20}$ When the molten mixture was used as a solvent for the metathesis of gallium trichloride and lithium nitride, three issues were to be addressed. Firstly additional lithium chloride produced as the by-product of the metathesis reaction would alter the composition and consequently the melting point of the molten mixture. Therefore, a large amount of the molten mixture relative to the amount of gallium trichloride and lithium nitride was used in order to avoid the melting temperature being altered dramatically. Secondly, there was no surfactant to provide steric stabilization to nanoparticles that might form. It was anticipated that ionic species absorbed onto the nanoparticle surface would help stabilizing the particles (via electrostatic repulsion) prior to the introduction of surfactants. Thirdly, the rather high melting temperature of the molten salt may favour the thermal decomposition of gallium nitride during the metathesis reaction. It was hoped that the thermal decomposition of gallium nitride might be avoided by having it also dissolved in the molten salt existing as a mixture of gallium, nitride, lithium, chloride ions mixture. Gallium nitride crystals were expected to crystallize out later when the solution was cooled.

In this experiment the lithium chloride/potassium chloride mixture was prepared by grinding lithium chloride and potassium chloride powder in a mortal. The mixture was 
heated in an oven to keep dry before use. It was found that at $\sim 370{ }^{\circ} \mathrm{C}$, the mixture formed a liquid which coexisted with a small amount of white solid. The coexistence of the liquid and the solid reflected that the mole ratio was not that of eutectic. It was nevertheless tried as a solvent for the metathesis reaction. To a solution of lithium nitride dissolved in a molten lithium chloride/potassium chloride mixture at $\sim 370{ }^{\circ} \mathrm{C}$ was added a powder of gallium trichloride. A bright orange flame was observed for less than one second. A light grey precipitate was isolated after extensive purification and was analysized in the TEM. From TEM the precipitate was found to be constituted of nanowires (figure 3.170) and nanoparticles which appeared quadrilateral (figure 3.171).
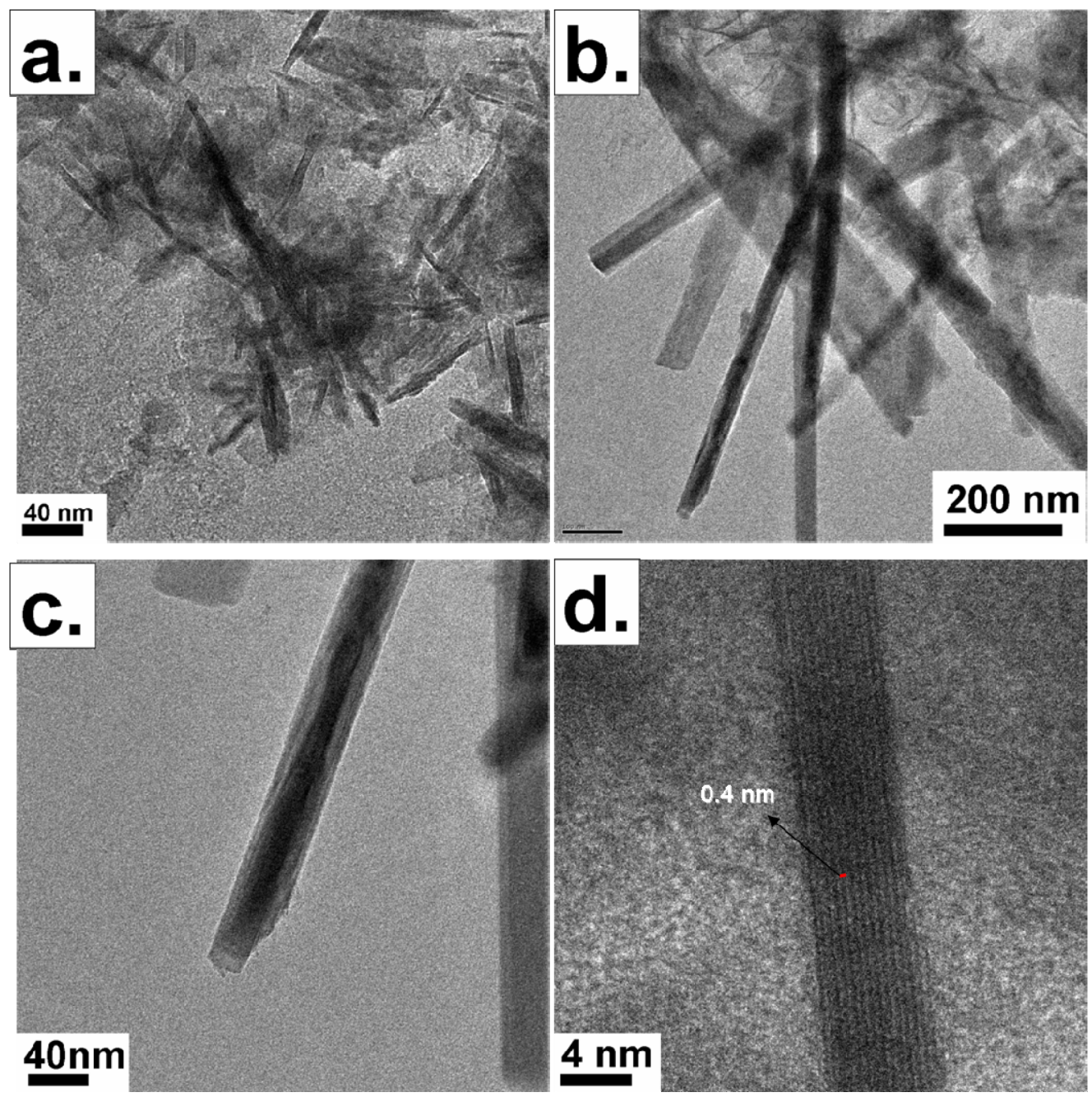

Figure 3.170 TEM images (a) nanowires covered in coating; (b) a group of nanowires; (c) an individual nanowire and (d) high resolution image showing atomic lattice fringes of a nanowire measured to $\sim 0.4 \mathrm{~nm}$. 
Chapter 3
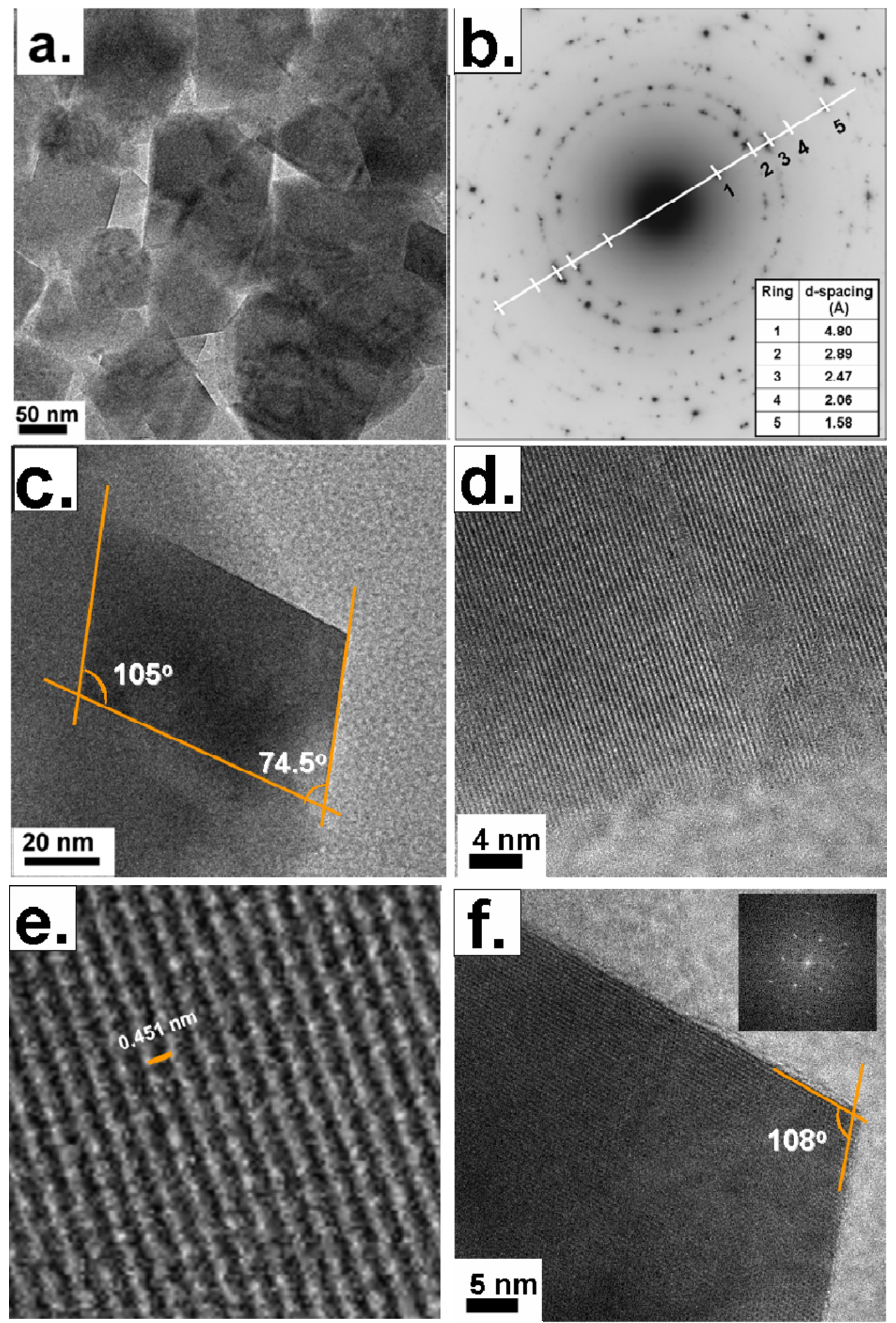

Figreu 3.171 TEM images (a) a group of nanoparticles appeared as quadrilaterals; (b) SAED pattern correspondent to nanoparticles shown in a; (c) a single highly faceted nanoparticle; (d) HRTEM image of the quadrilateral nanoparticle; (e) atomic lattice fringes observed for a quadrilateral nanoparticle; (f) a HRTEM image shows atomic lattice fringes of a nanoparticle appeared as a quadrilateral. 
The nanowires were found to be coated by a layer of light contrast material (figure $3.170 \mathrm{a}$ and $\mathrm{b}$ ) which from SAED and EDS were likely to be residue lithium chloride/ potassium chloride. The nanowires have diameters ranged between 5 to $40 \mathrm{~nm}$ and lengths ranged between 40 to $400 \mathrm{~nm}$. One of the nanowire (figure $3.170 \mathrm{~d}$ ) has lattice spacing of $\sim 0.4 \mathrm{~nm}$. The composition of the nanowires is unclear at the time being.

The nanoparticles which appeared as quadrilaterals under TEM (figure a,c,f) were highly crystalline and most of them were single crystals. One of the nanoparticle have obtuse angles measured to be $105-108^{\circ}$ and acute angles measured to be $74.5^{\circ}$ (figure $3.171 \mathrm{c})$. The lattice spacing of one of the quadrilateral was estimated to be $0.45 \mathrm{~nm}$ (figure 3.17e). This agreed well with the d-spacing of $0.48 \mathrm{~nm}$ calculated from the SAED pattern (figure 3.171b). From EDS, the nanoparticles were found to be consisted of gallium, carbon and oxygen and silicon. No nitrogen was observed during the EDS analysis. The silicon signal was believed to originate from instrumentation contamination. The actual composition of the material could not be elucidated with confidence from the evidences available. A possible candidate - rhombohedral lithium gallium oxide $\left(\mathrm{LiGaO}_{2}\right)$ - was suggested for the calculated d-spacings matched those of a reference lithium gallium oxide ${ }^{21}$. However, due to disagreements in diffraction intensities (when the SAED pattern was compared the reference diffraction pattern of $\mathrm{LiGaO}_{2}$ ) it was rejected as a potential candidate. 


\subsection{Thermal Decompositions of Organometallic Precursors Containing Gallium-Nitrogen Bonds}

Reported research work in the synthesis of colloidal gallium nitride nanoparticles in solution from coordination or organometallic compounds has been scarce. ${ }^{7,9,22,23}$ To produce colloidal gallium nitride nanoparticles close to the Bohr radius of gallium nitride $(2.5 \mathrm{~nm})$ solution phase synthesis is the most promising method.

The first colloidal synthesis of gallium nitride nanoparticles was reported by Mićić and coworkers in 1999. ${ }^{7}$ The precursor employed was a polymeric gallium imide $\left\{\mathrm{Ga}(\mathrm{NH})_{3 / 2}\right\}_{\mathrm{n}}$ which was prepared from reacting ammonia with a dimeric amidogallane of the formula $\mathrm{Ga}_{2}\left(\mathrm{NMe}_{2}\right)_{6}$. The yield of gallium nitride nanoparticles was low (several percent) and size control was difficult due to poor solubility of the polymeric precursor.

In 2004, Sarkar and Rao prepared colloidal gallium nitride nanoparticles from solvothermal treatment of organogallium cupferron of the formula $\mathrm{Ga}\left(\mathrm{C}_{6} \mathrm{H}_{5} \mathrm{~N}_{2} \mathrm{O}_{2}\right)_{3}$ and hexamethyldisilazane $\left(\mathrm{Me}_{3} \mathrm{SiNHSiMe}_{3}\right){ }^{22}$ A year later, Sarkar and coworkers reported the preparation of crystalline gallium nitride nanoparticles via refluxing an urea complex of gallium trichloride $\left[\mathrm{Ga}\left(\mathrm{H}_{2} \mathrm{NCONH}_{2}\right)_{6}\right] \mathrm{Cl}_{3}$ in trioctylamine. ${ }^{23}$ However the reaction yield was not reported.

In literature, it has been suggested that gallium nitride nanoparticles may be prepared at relative low reaction temperatures $\left(<400{ }^{\circ} \mathrm{C}\right)$ if starting with a compound which contains pre-established Ga-N bonds. ${ }^{10}$ For example, Goodwin et. al. synthesized gallium nitride nanoparticles by thermally decomposing the compound $\left[(\mathrm{Me})_{2} \mathrm{GaN}\left(\mathrm{C}_{6} \mathrm{H}_{6}\right)_{2}\right]_{2}(\mathbf{1})$ in a silica gel matrix at $200{ }^{\circ} \mathrm{C} .{ }^{8}$ The nanoparticles synthesized from 1 however were poorly crystalline and a post-synthesis annealing at $\sim 500{ }^{\circ} \mathrm{C}$ under ammonia was required to improve the crystallinity of the nanoparticles.

Compound 1 has a 4-member core structure comprising of two Ga-N sigma bonds and two Ga-N dative bonds (figure 3.30). Such a core structure is crucial allowing formation of gallium nitride via thermal decomposition process at a temperature $<400{ }^{\circ} \mathrm{C}$. Two organometallic compounds $\left[\left(\mathrm{Me}_{3} \mathrm{C}\right)_{2} \mathrm{Ga}\left(\mu-\mathrm{NHNHCMe}_{3}\right)\right]_{2}(2)$ and 
$\left[\left(\mathrm{Me}_{2} \mathrm{~N}\right)_{2} \mathrm{Ga}\left(\mu-\mathrm{NMe}_{2}\right)\right]_{2}(3)$ were studied in this thesis. Compound 2 and 3 both have a 4-member core structure similar to that of compound 1 (figure 3.30). It was hoped that by thermal decompose compound 2 and 3 in coordinating solvents that colloidal crystalline gallium nitride nanoparticles could be produced.<smiles>CC(C)N1C(C)(C)N(c2ccccc2)C(C)(C)C1(C)c1ccccc1</smiles>

1<smiles>CC(C)C([Y](NNC(C)(C)C)NC(C)(C)C)(C(C)(C)C)C(C)(C)C</smiles>

2

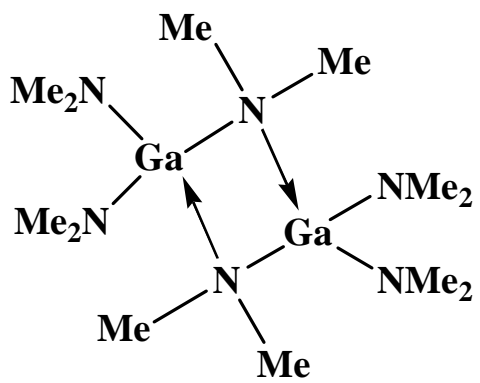

3

Figure 3.30 Schematic presentations of compounds 1-3. All three compounds have similar core structures.

\subsection{Synthesis and Thermal Decomposition of Bis[di(tert- butyl)gallium (tert-butyl-hydrazide)] $\left.\left[\left(\mathrm{Me}_{3} \mathrm{C}\right)_{2} \mathrm{Ga}(\mu-\mathrm{NHNHCMe})_{3}\right)\right]_{2}$ (Experiment 11)}

In this experiment, compound $2\left[\left(\mathrm{CMe}_{3}\right)_{2} \mathrm{Ga}\left(\mu-\mathrm{NHNHCMe}_{3}\right)\right]_{2}$ was synthesized and subjected to a thermal decomposition reaction. Compound 2 was prepared from modified literature methods. ${ }^{24,25,26}$ Compound $\mathbf{2}$ when compared to compound $\mathbf{1}$ was expected to thermally decompose more readily due to the bridging ligand in compound 2 being a hydrazide $\left(-\mathrm{NH}-\mathrm{NHCMe}_{3}\right)$ instead of that of a tertiary amide $\left(-\mathrm{N}\left(\mathrm{C}_{6} \mathrm{H}_{6}\right)_{2}\right)$ in compound 1. The total bond enthalpies calculated for cleaving two moles of N-C bonds is larger than that of breaking a N-H and a N-N bonds. The calculation was performed based on average bond enthalpies quoted in SI Chemical Data book by Aylward and Findlay. ${ }^{16}$ 


\section{Part 1: Synthesis and Characterization of Compound 2 $\left[\left(\mathrm{Me}_{3} \mathrm{C}\right)_{2} \mathrm{Ga}\left(\mu-\mathrm{NHNHCMe}_{3}\right)\right]_{2}$}

The title compound was synthesized following closely the method reported by Ulh and Emden (scheme 1). ${ }^{24}$ In Ulh and Emden's report, ditert-butyl gallium chloride was treated with a tert-butylhydrazine to form an adduct (reaction II, scheme 1) which was deprotonated with tert-butyllithium (reaction III, scheme 1) to form the title hydrazide (2). In this experiment Ulh and Emden's method was modified in 3 ways. Firstly, ditertbutyl gallium chloride $\left[\left(\mathrm{Me}_{3} \mathrm{C}\right)_{2} \mathrm{GaCl}\right]$ was synthesized following a method reported by Kovar and coworkers ${ }^{25}$ instead of Hoffman's ${ }^{27}$ method which was employed by Ulh and Emden. Secondly, the three steps synthesis was combined into a one step synthesis in which intermediate compounds were not isolated. Thirdly, solid tert-butyl hydrazine hydrochloric acid adduct was used instead of liquid tert-butyl hydrazine. The acid adduct was treated with tert-butyl lithium at room temperature half an hour before use in order to prepare fresh tert-butyl hydrazine.

I. $\mathrm{GaCl}_{3}+2 \mathrm{Me}_{3} \mathrm{CLi} \longrightarrow\left(\mathrm{Me}_{3} \mathrm{C}\right)_{2} \mathrm{GaCl}+2 \mathrm{LiCl}$

II.<smiles>CC(C)[C@@](Cl)(NNC(C)(C)C)C(C)(C)C</smiles>

III.

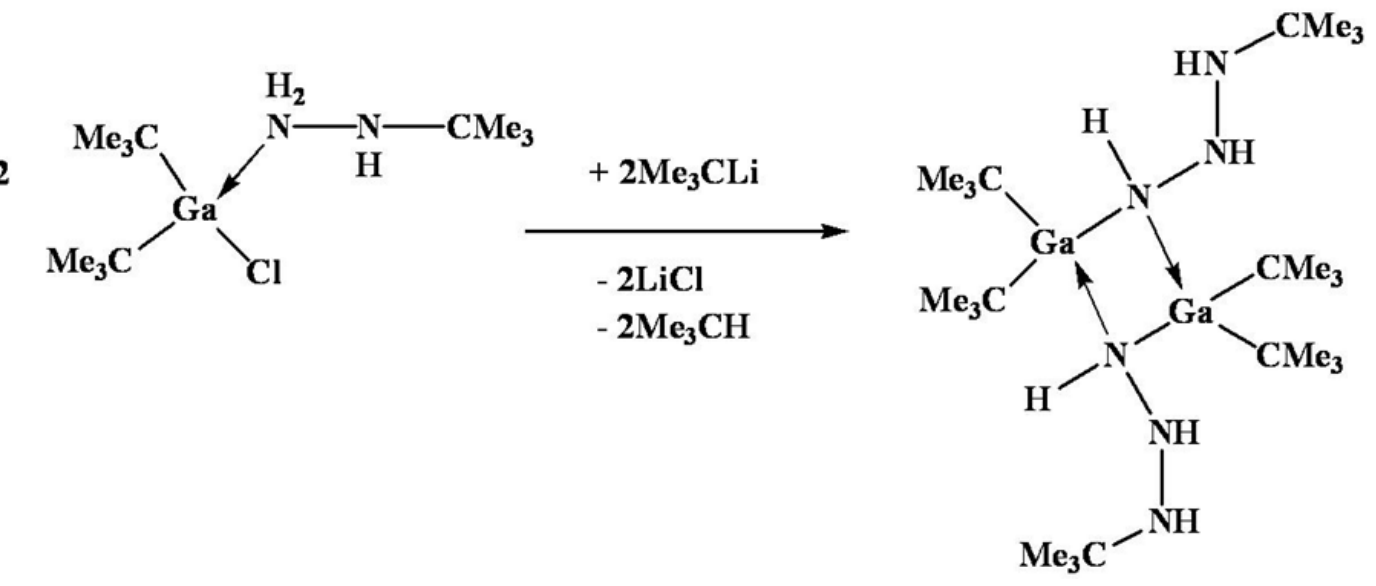

Scheme 3.310 Three-step synthesis of compound $2\left[\left(\mathrm{Me}_{3} \mathrm{C}\right)_{2} \mathrm{Ga}\left(\mu-\mathrm{NHNHCMe}_{3}\right)\right]_{2}$. 
Table 3.311 ${ }^{1} \mathrm{H}$ NMR data of compound 2. Chemical shifts correspond to atoms shown bold italic text. Resonance mismatch was highlighted in grey.

\begin{tabular}{|c|c|c|}
\hline & $\begin{array}{c}\text { Experiment } \\
\left(\mathrm{C}_{6} \mathrm{D}_{6}, 500 \mathrm{MHz}, 293 \mathrm{~K}\right)\end{array}$ & $\begin{array}{c}\text { Reference } 24 \\
\left(\mathrm{C}_{6} \mathrm{D}_{5} \mathrm{CD}_{3}, 400 \mathrm{MHz}, 300 \mathrm{~K}\right)\end{array}$ \\
\hline$\left(\boldsymbol{H}_{3} \mathrm{C}\right)_{3} \mathrm{C}-\mathrm{Ga}$ & $1.31(\mathrm{~s})$ & $1.31(\mathrm{~s})$ \\
\hline$\left(\boldsymbol{H}_{3} \mathrm{C}\right)_{3} \mathrm{C}-\mathrm{N}$ & $0.60(\mathrm{~s})$ & $0.94(\mathrm{~s})$ \\
\hline $\boldsymbol{H}-\mathrm{NC}\left(\mathrm{CH}_{3}\right)_{3}$ & $2.79(\mathrm{~s}, \mathrm{br})$ & $2.85(\mathrm{~s}, \mathrm{br})$ \\
\hline $\boldsymbol{H}-\mathrm{N}-\mathrm{Ga}$ & $3.61(\mathrm{~s}, \mathrm{br})$ & $3.61(\mathrm{~s}, \mathrm{br})$ \\
\hline
\end{tabular}

Note: $\mathrm{s}$, br represent singlet and broad.

Table 3.312 ${ }^{13} \mathrm{C}$ NMR data of compound 2. Data correspond to bold italic text. Resonance mismatch was highlighted in grey.

\begin{tabular}{|l|l|l|}
\hline & \multicolumn{1}{|c|}{$\begin{array}{c}\text { Experiment } \\
\left(\mathrm{C}_{6} \mathrm{D}_{6}, 500 \mathrm{MHz}, 293 \mathrm{~K}\right)\end{array}$} & $\begin{array}{c}\text { Reference } 24 \\
\left(\mathrm{C}_{6} \mathrm{D}_{6}, 100 \mathrm{MHz}, \mathrm{N} / \mathrm{A}\right)\end{array}$ \\
\hline$\left(\mathrm{CH}_{3}\right)_{3} \mathrm{C}-\mathrm{Ga}$ & 32.90 & 33.0 \\
\hline $\boldsymbol{C}\left(\mathrm{CH}_{3}\right)_{3}-\mathrm{Ga}$ & 25.60 & 25.2 and 26.6 \\
\hline$\left(\boldsymbol{C} \mathrm{H}_{3}\right)_{3}-\mathrm{C}-\mathrm{N}$ & Missing & 27.1 and 27.3 \\
\hline $\boldsymbol{C}\left(\mathrm{CH}_{3}\right)_{3}-\mathrm{N}$ & 54.50 & 54.2 and 54.4 \\
\hline
\end{tabular}<smiles>CC(C)(C)NN[Y](NNC(C)(C)C)C(C(C)(C)C)(C(C)(C)C)[Ge](C)(C)C</smiles>

Figure 3.313 Compound 2 with the methyl groups of the tert-butyl moieties shown in red. From ${ }^{1} \mathrm{H}$ and ${ }^{13} \mathrm{C}$ NMR, the methyl groups were not present in compound $\mathrm{S} 2$. 
A compound (S2) isolated after purification was studied by ${ }^{1} \mathrm{H}$ and ${ }^{13} \mathrm{C} \mathrm{NMR}$ spectroscopy. Compound S2 was found to exhibit ${ }^{1} \mathrm{H}$ and ${ }^{13} \mathrm{C}$ resonances similar to those of compound 2. The ${ }^{1} \mathrm{H}$ and ${ }^{13} \mathrm{C}$ resonances of compound $\mathrm{S} 2$ due to its core structure matched well with those arising from the core structure of compound 2. Therefore compound S2 and compound 2 were expected to have the same core structure (in black in figure 3.313). The ${ }^{1} \mathrm{H}$ and ${ }^{13} \mathrm{C}$ resonances of compound 2 due to three methyl groups (in red in figure 3.313) were not observed in compound S2. Instead of a singlet at $0.94 \mathrm{ppm}$ assigned to $\left(\boldsymbol{H}_{3} \mathrm{C}\right)_{3} \mathrm{C}-\mathrm{N}$ a singlet at $0.60 \mathrm{ppm}$ was observed in the ${ }^{1} \mathrm{H}$ NMR of compound S2. In addition to that the carbon resonance due to $\left(\mathrm{CH}_{3}\right)_{3}-\mathrm{C}-\mathrm{N}$ was absent in the ${ }^{13} \mathrm{C}$ NMR spectrum of compound S2. Therefore it was proposed that in compound S2, the three methyl groups had been placed by three protons.

\section{Part 2: Attempted Synthesis of Gallium Nitride via Thermal Decomposition of Compound S2}

The thermal decomposition reaction of compound S2 was studied. It was hoped that compound S2 would thermally decomposed to produce gallium nitride. A solution of compound S2 dissolved in trioctylamine was injected into a liquid of trioctylamine preheated to $344{ }^{\circ} \mathrm{C}$ under a dry ammonia atmosphere. Ammonia atmosphere was used because the thermal decomposition of compound 1 which produeced gallium nitride nanoparticles was conducted under ammonia. ${ }^{8}$ The mixture was allowed to react for 10 hours and a white solid precipitate was isolated after purification. 


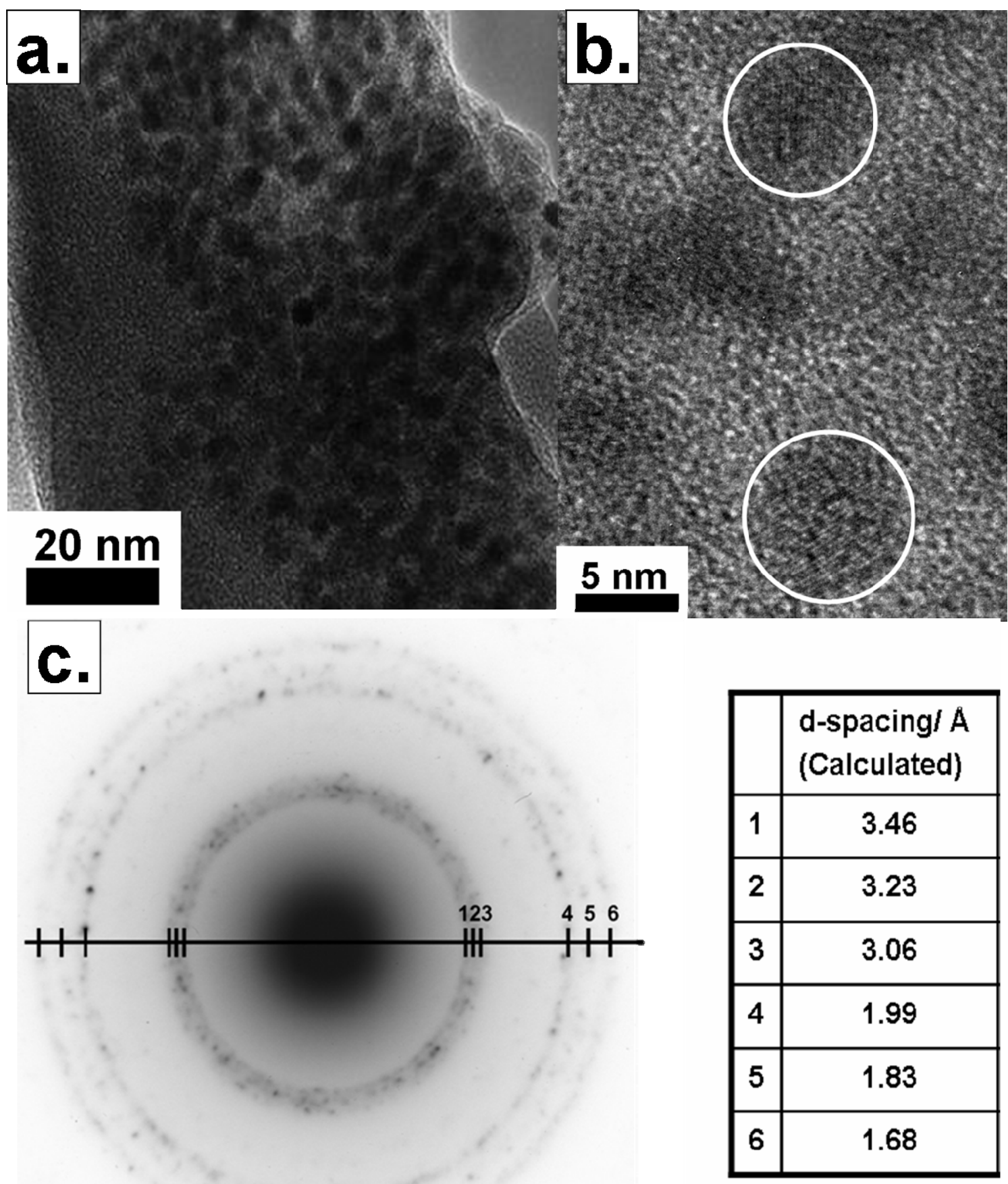

Figure 3.314 TEM images and SAED pattern. (a) a group of nanoparticles of $5 \mathrm{~nm}$ in size; (b) nanoparticles with lattice fringes and (c) SAED pattern of the nanoparticles. 
The white solid was studied under TEM. As may be seen in figure 3.314, the solid was constituted of monodispersed nanoparticles of $\sim 5 \mathrm{~nm}$ in size. Figure $3.314 \mathrm{~b}$ shows that the nanoparticles were highly crystalline and atomic lattice fringes may be observed. From SAED, only one type of crystalline compound was present. Analysis of the SAED diffraction suggested that the nanoparticles adopt the wurtzite structure. The d-spacings determined however were found to be significantly larger than those of wurzite gallium nitride (table 3.315 ). In light of this the material could not be confidently identified to be gallium nitride. When indexed to the wurtzite structure the lattice parameters $a$ and $c$ of the material were calculated to be $3.99 \AA$ and $6.46 \AA$ using equation 3.1 derived from reference 28. The values of $a$ and $c$ calculated for the material were $27 \%$ larger than those of wurtzite gallium nitride. ${ }^{29}$ No sufficient amount of the nanoparticles were available for further XRD characterization.

$$
\frac{1}{4 d^{2}}=\frac{\left(h^{2}+h k+l^{2}\right)}{3 a^{2}}+\frac{l^{2}}{4 c^{2}}---- \text { equation } 3.1
$$

Table 3.315 A list of calculated d-spacings and Miller indexes.

\begin{tabular}{|c|c|c|c|}
\hline $\begin{array}{c}\text { Nanoparticles } \\
\text { d-spacing } \\
\AA\end{array}$ & $\begin{array}{c}\text { Nanoparticles } \\
\text { d-spacing (converted) }\end{array}$ & $\begin{array}{c}\text { Wurtzite GaN } \mathbf{2 9}^{29} \\
\mathbf{d - s p a c i n g} \\
\AA\end{array}$ & $\begin{array}{c}\text { Miller } \\
\text { Indexes }\end{array}$ \\
\hline 3.46 & 2.72 & 2.724 & 100 \\
\hline 3.23 & 2.54 & 2.562 & 002 \\
\hline 3.06 & 2.40 & 2.405 & 101 \\
\hline 1.99 & 1.56 & 1.570 & 102 \\
\hline 1.83 & 1.46 & 1.440 & 110 \\
\hline 1.68 & 1.32 & 1.360 & 103 \\
\hline
\end{tabular}




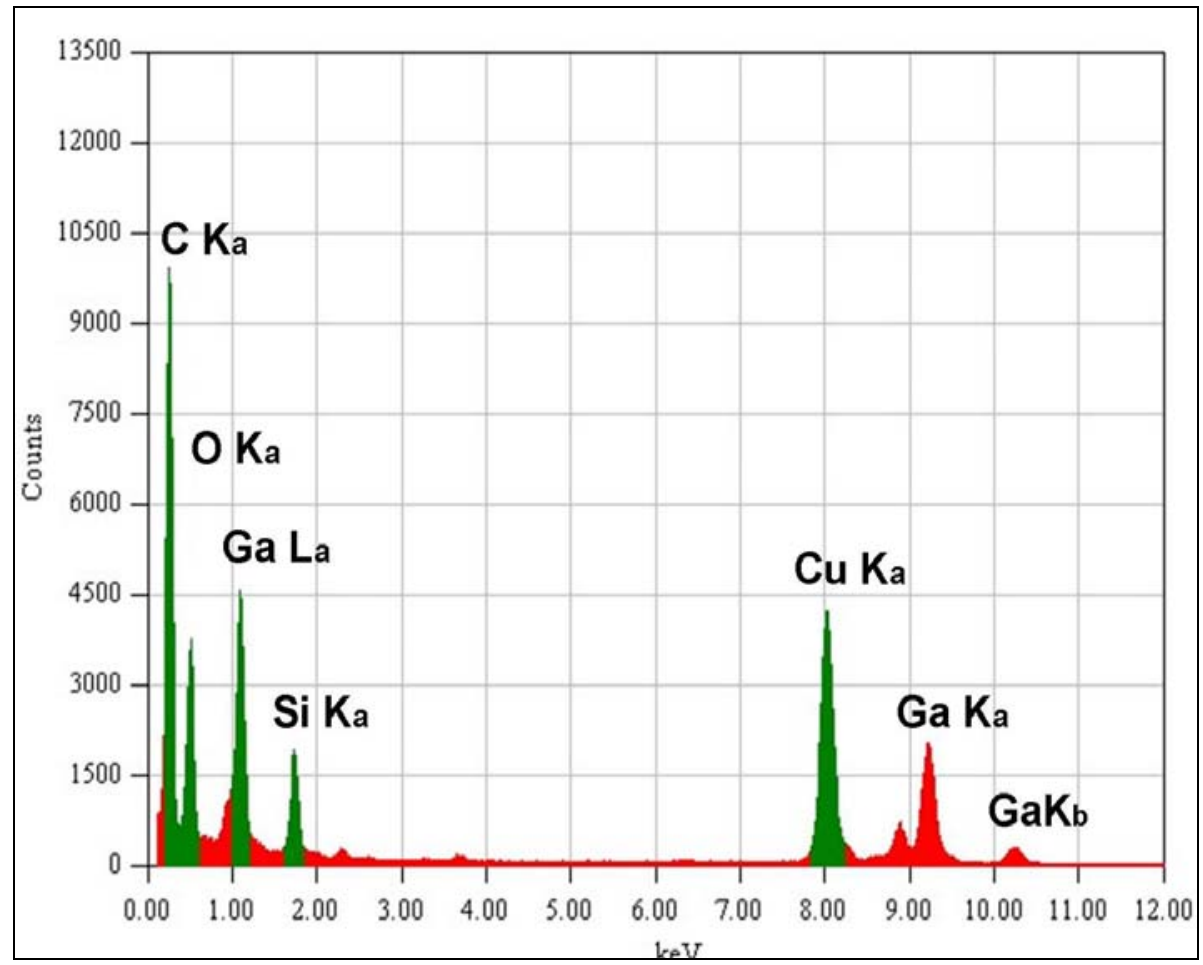

Figure 3.316 An EDS spectrum suggested that the nanoparticles were composed of mainly gallium oxygen and silicon.

EDS analysis (figure 3.316) showed that the nanoparticles contained gallium, silicon and oxygen. No nitrogen was detected. The presence of silicon and oxygen may be explained by the contamination due to silicone-based grease used during the synthesis. The reaction mixture has been refluxed at $>300{ }^{\circ} \mathrm{C}$ for at least 7 hours and a small amount of silicone-based grease may have been dissolved into the reaction mixture. There is no firm conclusion at the time being in regard to the chemical identity of the nanoparticles. 


\subsection{Attempted Synthesis and Thermal Decomposition of Tris(dimethyl-amino)gallane $\left[\left(\mathrm{Me}_{2} \mathrm{~N}\right)_{2} \mathrm{Ga}\left(\mu-\mathrm{NMe}_{2}\right)\right]_{2}$ (Experiment 12)}

\section{Part I: Attempted Synthesis of Compound $3\left[\left(\mathrm{Me}_{2} \mathrm{~N}\right)_{2} \mathrm{Ga}\left(\mu-\mathrm{NMe}_{2}\right)\right]_{2}$}

In the year 1996 Gonslaves and coworkers prepared crystalline gallium nitride as a powder by heating the compound $\left[\left(\mathrm{NMe}_{2}\right)_{2} \mathrm{Ga}\left(\mu-\mathrm{NMe}_{2}\right)\right]_{2}$ at $600{ }^{\circ} \mathrm{C}$ under ammonia atmosphere. ${ }^{10 \mathrm{c}}$ The powder consisted of agglomerated gallium nitride with an average domain size of $5 \mathrm{~nm}$. The particles were then sonicated and dispersed in poly(methyl metacryate) in order to keep the particles segregated. A decade later, Pan and coworkers reported the preparation of colloidal gallium nitride nanoparticles of $5 \mathrm{~nm}$ in size via solution phase thermal decomposition of the same compound. ${ }^{9}$ However, the particles were poorly crystalline and no lattice fringes were observed under HRTEM conditions. The purpose of this experiment was to reproduce Pan's synthesis. Once reproduced, modifications would be applied in the hope to improve the crystallinity of the nanoparticles.

I. $\mathrm{GaCl}_{3}+3 \mathrm{Me}_{2} \mathrm{NLi}$

$\left(\mathrm{Me}_{2} \mathrm{~N}\right)_{3} \mathrm{Ga}+3 \mathrm{LiCl}$

II.

$$
\left(\mathrm{Me}_{2} \mathrm{~N}\right)_{3} \mathrm{Ga}
$$

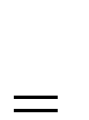<smiles>CN(C)[CH][NH3+]</smiles>

III.

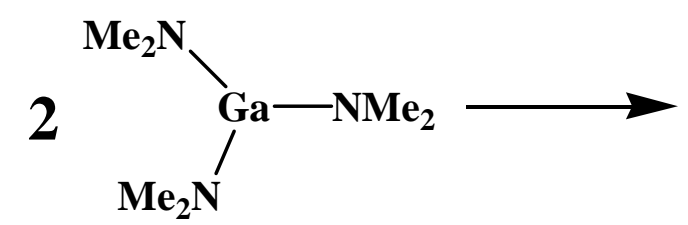<smiles>CN(C)C1[C@H](N(C)C)N(C)[C@@H](N(C)C)N1C</smiles>

Scheme 3.32 Two-step synthesis of $\left[\left(\mathrm{NMe}_{2}\right)_{2} \mathrm{Ga}\left(\mu-\mathrm{NMe}_{2}\right)\right]_{2}$ in anhydrous hexane. 
Gallium trichloride and lithium dimethylamide were allowed to react in a 1:3 ratio in anhydrous hexane according to a method reported by Pan and coworkers (scheme 3.32). ${ }^{9}$ A white solid was isolated from the two-step reactions. In their report, Pan and coworkers claimed that the yield of compound 3 was high but no quantitative yield was given. In the ${ }^{1} \mathrm{H}$ NMR of compound 3 in deuterated benzene $\left(\mathrm{C}_{6} \mathrm{D}_{6}\right)$, two resonances appearing at $2.4 \mathrm{ppm}$ and $2.82 \mathrm{ppm}$ as two singlets due to $\left(\mathrm{N}\left(\mathrm{CH}_{3}\right)_{2}\right)$ and $\mu-\mathrm{N}\left(\mathrm{CH}_{3}\right)_{2}$ were expected. ${ }^{26}$ The two resonances were observed in the ${ }^{1} \mathrm{H}$ NMR spectrum of the white solid but only with very low intensities. No quantitative yield was determined due to the difficulty to isolate compound 3 from the relatively large quantity of by-products. The by-products were found to be consisted of two different species which might be isomers of each other due to they displayed very similar ${ }^{1} \mathrm{H}$ resonances in their ${ }^{1} \mathrm{H}$ NMR spectra. When cooled to $-20{ }^{\circ} \mathrm{C}$, one of the by-products precipitated out from the other. No further investigations into the compositions of the by-products were attempted.

\section{Part II : Attempted Thermal Decomposition of Compound 3}

Despite the failure to synthesize compound 3 in appreciable yield, the products formed in the synthesis were subjected to a thermal decomposition reaction. The purpose of carrying out the reaction was to examine if gallium nitride nanoparticles could be produced. Pan and coworkers prepared gallium nitride nanoparticles by heating compound 3 in $5 \mathrm{~mL}$ of trioctylamine/hexadecylamine mixture to reflux at $330-350{ }^{\circ} \mathrm{C}$ for three days. They found that in the absence of hexadecylamine no gallium nitride nanoparticles were formed. They attributed that to the transamination reaction between hexadecylamine and the amide moieties in compound 3. The attempt to reproduce Pan's thermal decomposition experiment was hampered by two experimental difficulties. Firstly it was difficult to keep the volume of the trioctylamine/hexadecylamine mixture under refluxing condition constant for three days. In a typical experiment, half of the solvent mixture was already lost after a day. The whole mixture became nearly dried out and a black viscous liquid formed from a white suspension after being heated for two day. Secondly the reaction temperature could not be kept constant due to the formation of a volatile species that had a boiling point $\sim 100{ }^{\circ} \mathrm{C}$ lower than the intended reaction temperature of $330-350{ }^{\circ} \mathrm{C}$. In one experiment a strongly coloured solution formed instead of a black viscous liquid. The solution appeared green and red at the same time indicating a very unique optical property. This liquid was very similar to the one isolated in experiment 6 in which hexadecylamine trioctylamine mixture was also used 
as a solvent. It was unclear whether Pan's and coworkers had had encountered the same technical difficulties. It was proposed pure compound 3 should be employed for the thermal decomposition reaction in pure hexadecyamine without the presence of trioctylamine.

\subsection{Conclusions}

Research work aiming at preparing gallium nitride nanoparticles from the metathesis reaction of gallium trichloride and lithium nitride in solution was unsuccessful. Various types of nanostructures including amorphous nanoparticles, crystalline nanorods, crystalline nanowires and quadrilateral nanoparticles were synthesized. In experiments in which benzene, trioctylamine, hexadecylamine were used as solvents, amorphous nanoparticles were produced. The amorphous nanoparticles were believed to be gallium metal nanoparticles formed from the metathesis reaction. It was proposed that the sparse solubility of lithium nitride in the solvents and the heat produced from the metathesis reaction worked to disfavour the formation of gallium nitride. In experiments in which polar solvents including dibenzofuran and lithium chloride/potassium chloride eutectic mixture were employed highly crystalline species nanorods, nanowire and nanoparticles of quadrilateral shape were produced. Most of the crystalline materials could not be conclusively identified apart from gallium oxide isolated as nanorods. All nanostructures characterized contained gallium and no nitrogen in general. An organometallic complex (compound S2) very similar to compound $2\left[\left(\mathrm{Me}_{3} \mathrm{C}\right)_{2} \mathrm{Ga}(\mu-\right.$ $\left.\left.\mathrm{NHNHCMe}_{3}\right)\right]_{2}$ was synthesized. The thermal decomposition of compound S2 produced crystalline nanoparticles of uniform size. The nanoparticles were found to adopt a wurtzite structure and have lattice parameters significantly larger than that of wurtzite gallium nitride. Organometallic precursor compound $3\left[\left(\mathrm{Me}_{2} \mathrm{~N}\right)_{2} \mathrm{Ga}\left(\mu-\mathrm{NMe}_{2}\right)\right]_{2}$ were synthesized according to literature method but only in very low yield and no thermal decomposition reaction of compound 3 could be carried out. It was believed that the literature method employed was not reproducible. 


\subsection{Experimental}

All reactions were carried out under a nitrogen atmosphere. All reagents were purchased from Sigma-Aldrich and were of the analytical grade unless otherwise mentioned. Solvents used for reactions were either purchased as anhydrous solvents or have been heated under vacuum over $100{ }^{\circ} \mathrm{C}$ for 1 hour to remove air and water.

\section{The Reaction of Gallium Trichloride and Lithium Nitride in Trioctylamine (Experiment 4)}

In a dry box, $0.1637 \mathrm{~g}$ of gallium trichloride was combined with $5 \mathrm{~mL}$ of trioctylamine in a three neck flask fitted with a Liebig condenser, a glass temperature probe housing and a pressure-equalizing dropping funnel. The dropping funnel contained a suspension of $0.0346 \mathrm{~g}$ of lithium nitride in $1 \mathrm{~mL}$ of trioctylamine. The mixtures were taken out from the dry box. The gallium trichloride/trioctylamine mixture was heated to $\sim 304{ }^{\circ} \mathrm{C}$ to form a clear pale yellow solution. To the yellow solution was added within a second all of the lithium nitride/tricotylamine mixture. The resulting mixture was allowed to react for $\sim 25$ minutes. A black solid precipitate was observed. The solution mixture was allowed to cool to $170{ }^{\circ} \mathrm{C}$ and $50 \mathrm{~mL}$ of methanol was injected. The resulting mixture contained a cloud white solution and a yellowish-white solid precipitate.

\section{The Reaction of Gallium Trichloride and Lithium Nitride in Hexadecylamine (Experiment 5)}

In a dry box, $0.3182 \mathrm{~g}$ of gallium trichloride, $0.0532 \mathrm{~g}$ of lithium nitride and $2.78 \mathrm{~g}$ of hexadecylamine were combined in a three neck flask equipped with a Liebig condenser, a glass temperature probe housing and a rubber septum. The mixture was taken out of the dry box and heated with stirring to $230{ }^{\circ} \mathrm{C}$. A dark brown solution initially observed turned into a greyish cloudy solution with a white solid precipitate. The mixture was heated at $230{ }^{\circ} \mathrm{C}$ for an overall 30 minutes. $2 \mathrm{~mL}$ of the solution mixture was syringed out and quenched with $\sim 10 \mathrm{~mL}$ of methanol. A white precipitate was observed.

\section{Purification}

The $\sim 12 \mathrm{~mL}$ solution was centrifuged at $\sim 3000 \mathrm{rpm}$ for 1.5 minutes to isolate a greyish to blackish solid precipitate. The solid precipitate was washed with $12 \mathrm{~mL}$ of methanol and centrifuged at $300 \mathrm{rpm}$ for 1.5 minutes. The process was repeated three times to 
isolate a greyish black solid precipitate. The final precipitate was re-suspended in $\sim 10$ $\mathrm{mL}$ of toluene.

\section{The Reaction of Gallium Trichloride and Lithium Nitride in Hexadecylamine /Trioctylamine Mixture (Experiment 6)}

\section{Part 1}

To $0.1744 \mathrm{~g}$ of lithium nitride in a round bottom flask was added $10 \mathrm{~mL}$ of gallium trichloride hexane solution $(0.09 \mathrm{~g} / \mathrm{mL})$. The mixture was pumped under vacuum to remove hexane and a dark grey solid mixture. In a dry box, to the grey mixture was added $3 \mathrm{~mL}$ of trioctylamine and the resulting mixture was stirred until in use.

\section{Part 2}

In a 3 neck flaks equipped with a Liebig condenser, a glass temperature probe housing and a rubber septum $3 \mathrm{~g}$ of hexadecylamine was heated to melt and pumped vacuum to remove air for 5 minutes before backfilled with nitrogen gas. The clear colourless liquid of hexadecylamine was heated to $200{ }^{\circ} \mathrm{C}$ and to it was added the final mixture prepared in section 1. A grey solution formed upon mixing. The grey solution was heated heated at $\sim 180{ }^{\circ} \mathrm{C}$ for 5 hours followed by $307{ }^{\circ} \mathrm{C}$ for 6 hours to produce a wet deep brown solid with a small amount of liquid. To the wet solid at $100{ }^{\circ} \mathrm{C}$ was added $50 \mathrm{~mL}$ of ethanol and a jade green/orange red solution was obtained.

\section{The Reaction of Gallium Trichloride and Lithium Nitride in Dibenzofuran (Experiment 7)}

In a dry box, a mixture containing $0.3 \mathrm{~g}$ of gallium trichloride, $0.058 \mathrm{~g}$ of lithium nitride and $3 \mathrm{~mL}$ of trioctylamine was heated at $50{ }^{\circ} \mathrm{C}$ under a nitrogen atmosphere. In a 3-neck flask, $9 \mathrm{~g}$ of dibenzofuran was heated to $208{ }^{\circ} \mathrm{C}$ in nitrogen. To dibenzofuran was added dropwise the suspension of lithium nitride in trioctylamine. A $30{ }^{\circ} \mathrm{C}$ temperature drop was observed after all the trioctylamine mixture was added. The resulting solution was heated to $280{ }^{\circ} \mathrm{C}$. After being at $\sim 280{ }^{\circ} \mathrm{C}$ for 4 hours, a yellow brown solution was obtained. After allowed to cool to $\sim 100{ }^{\circ} \mathrm{C}, 15 \mathrm{~mL}$ of absolute ethanol was injected to the brown solution. 


\section{Purification}

The resulting ethanoic mixture was diluted with more ethanol. After allowed to settle, a brown solid was isolated.

\section{Reaction of Gallium Trichloride and Lithium Nitride in Molten Lithium Chloride/ Potassium Chloride Mixture (Experiment 10)}

\section{Part 1 Preparation of Lithium Chloride/ Potassium Chloride Mixture}

Hydrated potassium chloride (ground into a fine powder) and lithium chloride were dried in an oven at $300{ }^{\circ} \mathrm{C}$ overnight. The two solid powders were taken out and allowed to cool to room temperature in a dessicator. $4.9991 \mathrm{~g}$ of lithium chloride and $6.0594 \mathrm{~g}$ potassium chloride were quickly weighted out, mixed and ground together to mix. During the grinding process, the solid started absorbing water from the air and the surface became moist. The mixture was returned to an oven operating at $100{ }^{\circ} \mathrm{C}$ for overnight before the next grinding. The grinding-heating process was repeated at least 5 times. The mixture was stored in the oven until in use.

\section{Part 2 Reaction}

After allowed to cool in a dessicator to room temperature, $0.5418 \mathrm{~g}$ of lithium chloride/ potassium chloride mixture was quickly weighted in a 3-neck flask. The mixture was heated to $300{ }^{\circ} \mathrm{C}$ in vacuo for 20 minutes. The mixture was further heated to $365{ }^{\circ} \mathrm{C}$ to form a greyish mixture consisting of colourless liquid, grey solid and white solid. To the mixture at $370{ }^{\circ} \mathrm{C}$ was added $0.0262 \mathrm{~g}$ of lithium nitride powder .A black mixture of liquid and solid was observed at $400{ }^{\circ} \mathrm{C}$. To this was added $0.0850 \mathrm{~g}$ of gallium trichloride powder. A bright orange flame observed upon addition which disappeared after a few seconds. A white solid formed on top of the black mixture. The mixture was heated at $\sim 400{ }^{\circ} \mathrm{C}$ for $1 \mathrm{hr}$ and 40 minutes before allowed to cool down to room temperature.

\section{Purification}

To the grey/white solid was added $30 \mathrm{~mL}$ of isobutylamine (dried over anhydrous sodium sulphate and purged with dry nitrogen). The mixture was sonicated for 1 hour. The amine solution was discarded and to the remaining solid was added $30 \mathrm{~mL}$ of 
water. The aqueous mixture was sonicated for 5 minutes and centrifuged at $4000 \mathrm{rpm}$ for 5 minutes to isolate a greyish white solid.

\section{Synthesis and Thermal Decomposition of Compound S2 Similar to}

Compound 2 Ditert-butyl-gallium Hydrazide

\section{$\left[\left(\mathrm{Me}_{3} \mathrm{C}\right)_{2} \mathrm{Ga}\left(\mu-\mathrm{NHNHCMe}_{3}\right)\right]_{2}$ (Experiment 11)}

\section{Synthesis of Compound S2}

\section{Part 1}

To a solution of $0.4845 \mathrm{~g}$ of gallium trichloride in $28 \mathrm{~mL}$ of benzene (charged in a three neck flask) was added dropwise a solution of $3.2 \mathrm{~mL}$ of tert-butyllithium pentane solution in $47 \mathrm{~mL}$ of benzene (charged in an pressure-equalizing dropping funnel) over a period of 2 hours. The mixture was allowed to stir for another 2 hours before subjected to heating in an oil bath pre-heated at $70{ }^{\circ} \mathrm{C}$ for 6 hours. The resulting solution was allowed to cool to room temperature before pumped under vacuum to remove benzene and any volatile species. To the remaining was added $20 \mathrm{~mL}$ of toluene anhydrous and the mixture was allowed to stir for $\sim 40$ minutes to form a brown solution.

\section{Part 2}

In a dry box, to a mixture containing $0.350 \mathrm{~g}$ of tert-butyl-hydrazine hydrochloride acid adduct, $\mathrm{HCl} \cdot \mathrm{HN}-\mathrm{NH}\left(\mathrm{CMe}_{3}\right)$, and $5 \mathrm{~mL}$ toluene was added $1.6 \mathrm{~mL}$ of $1.7 \mathrm{M}$ tertbutyllithium in pentane. The mixture was stirred for 35 minutes to form a yellowish solution.

\section{Part 3}

To the final brown solution obtained in section 1 was added the yellowish solution prepared in section 2. The resulting mixture was stirred for an hour and a yellowish brown solution obtained.

\section{Part 4}

The yellowish brown solution was cooled in a dry-ice/acetone bath. To the cold solution was added dropwise $1.6 \mathrm{~mL}$ of $1.7 \mathrm{M}$ tert-butyllithium in pentane. The resulting mixture was allowed to warm up to room temperature and was stirred overnight. The resulting 
brown mixture was filtered under nitrogen to isolate a clear yellow solution. A small amount of grey solid was observed on top of the filter.

\section{Thermal decomposition of Compound S2}

A mixture containing $1 \mathrm{~mL}$ of trioctylamine and $1 \mathrm{~mL}$ of the yellow solution isolated in part 4 was pumped under vacuum with constant stirring to remove toluene. The mixture was injected quickly into a liquid of trioctylamine pre-heated to $340{ }^{\circ} \mathrm{C}$ under ammonia. The mixture was heated at $\sim 320-330{ }^{\circ} \mathrm{C}$ for 7 hours followed by $\sim 250{ }^{\circ} \mathrm{C}$ for 3 hours. The resulting yellow solution was allowed to cool to room temperature under ammonia. The mixture was diluted with a $100 \mathrm{~mL}$ of methanol and the pale yellow solution was kept at $4{ }^{\circ} \mathrm{C}$ for 3 days to precipitate a white solid. The white solid was isolated and dissolved in toluene. 


\subsection{References}

1. (a) Nakamura, S.; Mukai, T.; Senoh, M.; Nagahama S., Iwasa N. J. Appl. Phys. Lett. 1993, 64, 1687-1689. (b) Nakamura, S.; Harada, Y.; Seno, M. Appl. Phys. Lett. 1991, 58, 2021-2023.

2. (a) Trodahl, H. J.; Budde, F.; Ruck, B. J.; Granville, S.; Koo, A.; Bittar, A. Appl. Phys. Lett. 2005, 97, 084309/1-084309/5. (b) Abernathy, C. R.; Mackenzie, J. D.; Donovan, S. M. J. Cryst. Growth 1997, 178, 74-86. (c) Nishinaga, T.; Narisuka, S. Cryst. Growth Tech. 2003, 55-92.

3. Wang, J.; Grocholl, L.; Gillan, E. G. Nano Lett. 2002, 2, 899-902.

4. McMurran, J.; Kouvetakis, J.; Nesting, D. C.; Smith, D. J.; Hubbard, J.L. J. Am. Chem. Soc., 1998, 120, 5233-5237.

5. Frank, A. C.; Fischer, R. A. Adv. Mater. 1998, 12, 961-964.

6. Janik, J. F.; Wells, R. L. Chem. Mater. 1996, 8, 82708-2711.

7. Mićić, O. I.; Ahrenkiel, S. P.; Bertram, D.; Nozik; A. J. Appl. Phys. Lett. 1999, $75,478-479$.

8. Goodwin, T. J.; Leppert, V. J.; Smith, C.A.; Risbud, S. H. Appl. Phys. Lett., 1996, 69, 3230-3231.

9. Pan, G.; Kordesch, M. E.; Van Patten, P. G. Chem. Mater. 2006, 18, 5392-5394. (c) Gonsalves, K. E.; Carlson, G.; Rangarajan, S. P.; Benaissa, M.; José-Yacamán; M. J. Mater. Chem. 1996, 6, 1451-1453. 
10. (a) Xie, Y.; Qian, Y.; Wang, W.; Zhang, S.; Zhang, Y. Science 1996, 272, 1926-1927. (b) Chen, C.; Liang, C. Tamkang. J. Sci. \& Engine. 2002, 5, 223-226.

11. Pan, G.; Kordesch, M. E.; Van Patten, P. G. Chem. Mater.2006, $18,3915-3917$.

12. (a) Wallace, C. H.; Reynolds, T. K.; Kaner, R. B. Chem. Mater. 1999, 11, 2299-2301. (b) Parkin, I. P. Chem. Soc. Rev., 1996, 25, 199-207.

13. http://www.sigmaaldrich.com/catalog/search/ProductDetail/ALDRICH/194905

14. (a) Pedersen, C. J. J. AM. CHEM. SOC. 1967, 89, 7017-7036.

(b) Pedersen, C. J.; Frensdorf, H. K. Angew. Chem. Int. Chem. (Engl.) 1972, $11,16-25$.

15. Data Retrieved from SciFinder Scholar. Calculated using Advanced Chemistry Development (ACD/Labs) Software V9.04 for Solaris (ㄷ 1994-2008 ACD/Labs)

16. (a) Battaglia, D.; Peng, X. Nano Letts. 2002, 2, 1027-1030.

(b) Lucey, D. W.; MacRae, D. J.; Furis, M.; Sahoo, Y.; Cartwright, A. N.; Prasad, P. N. Chem. Mater. 2005, 17, 3754-3762.

17. Aylward G.; Findley, T. SI CHEMICAL DATA $4^{\text {th }}$ Ed., John Wiley and Sons Australia, 1998.

18. Hu, J.Q.; Deng, B.; Zhang, W. X.; Tang, K. B.; Qian,Y. T. Chem. Phys. Lett. 2002, 351, 229-234

19. (a) http://www.sigmaaldrich.com/catalog/search/ProductDetail/ALDRICH/479330 (b)http://www.crct.polymtl.ca/fact/ 
20. (a) Kobayashi, F.; Ogawa, T.; Okamoto, Y.; Akabori, M. J. Alloys \& Compounds 1998, 271-273, 374-377. (b) Shirai, O.; Kato, T.; Iwai, T.; Arai, Y.; Yamashita, T. J. Phys. \& Chem. Solids 2005, 66, 456-460.

21. Marezio, M.; Remeika, J. P. Phys. Chem. Solids 1965, 26, 1277-1280.

22. Sardar, K.; Rao, C. N. R. Adv. Mater. 2004, 16, 425-429.

23. Sardar, K.; Dan, M.; Schwenzer, B.; Rao, C. N. R. J. Mater. Chem. 2005, 15, 2175-2177.

24. Uhl, W.; Emden, C. H J. Organomet. Chem. 2005, 690, 1529-1539.

25. Kovar, R. A.; Loaris, G.; Derr, H.; Callaway, J. O. Inorg. Chem. 1974, 13, 14761479.

26. Nöth, H.; Konrad, P. Z. Naturforsch. 1975, 30B, 681-687.

27. Hoffmann, G.; Hellert, D.; Warren, M. J. Organomet. Chem. 1993, 444, 21-24.

28. Ladd, M.; Palmer, R. Structure Determination by X-ray Crystallography $4^{\text {th }}$ Ed. Kluwer Academic/Plenum Publishers, New York, United States of America, 2003.

29. Miwa, K.; Fukumoto, A. Phys. Rev. B: Condens. Matter. 1993, 48, 7897-7902. 


\section{Chapter 4 Solution Synthesis of Indium Phosphide Nanowires Using Red Phosphorus}

This chapter describes the development of a new method to prepare indium phosphide nanowires. Red phosphorus was used as the only phosphorus source and it was reacted with either indium acetate or indium trichloride in the presence or absence of sodium borohydride.

Red phosphorus has been used in the preparation of bulk indium phosphide such as by the Czochralski methods at a temperature higher than $1000{ }^{\circ} \mathrm{C} .{ }^{1}$ Films of indium phosphide grown on substrates have also been prepared from red phosphorous via either $\mathrm{MOCVD}^{2}$ or $\mathrm{MBE}^{3}$ approaches $\sim 500-600{ }^{\circ} \mathrm{C}$. However no preparation of indium phosphide in any nanostructures from red phosphorus in solution at a reaction temperature lower than $300{ }^{\circ} \mathrm{C}$ at ambient pressure has been previously reported.

Indium phosphide nanowires were first prepared in solution by Trentler and coworkers. ${ }^{4}$ An organometallic indium compound for example trimethylindium was used as the indium source and tris-trimethylsilylphosphine was used as the phosphorus source. The reaction proceeded to give indium phosphide nanowires in the presence of nanoparticles of a low melting metal such as that of indium. The growth of nanowires was governed by the metal nanoparticles which have become molten liquid droplets at a reaction temperature higher than their melting points. The growth mechanism was coined solution-liquid-solid (SLS) growth and may be regarded an analogue of the VLS growth mechanism. ${ }^{4-6}$

Phosphines including tris-trimethylsilylphosphine are commonly used in the preparation of SLS grown indium phosphide nanowires. Most phosphines are highly toxic and pyrolytic and may be costly. These issues become particularly problematic when scaling up the syntheses. The motivation of the research was to establish a non-phosphine route to prepare indium phosphide nanowires via SLS growth. 
All experiments reported in this chapter were conducted using a 1:1 indium to phosphorus molar ratio. A 5 mole equivalent of sodium borohydride was used in experiments whenever applicable. The aim of this research was to develop a new preparative method and the research has been carried out in an exploratory manner. Five experiments crucial in the development of the new method were selected and the corresponding results were described in sections 4.1 to 4.4 . Indium acetate was used as the indium source in experiments 1 and 2 and indium trichloride was used in experiments 3-5. Experiment 1 and 2 differed in the coordinating strength of the solvents employed and experiment 4 and 5 differed only in reaction sequences that were detailed in table 4.53 (presented in section 4.5). Experiment 3 was a unique experiment which was conducted under conditions different from that of the other four. It was from the results of experiment 3 that the idea of preparing indium phosphide from indium trichloride, red phosphorus and sodium borohydride took shape. The addition of sodium borohydride was expected to create a reaction pathway that would lead to the formation of indium phosphide. Detailed discussions on the use of sodium borohydride are presented in section 4.5. Whenever applicable, the words "sodium borohydride" refers to a sodium borohydride suspension in trioctylamine. Solid sodium borohydride does not dissolve in trioctylphosphine to form a real solution but could be dispersed to form a suspension. A fine suspension is essential for the transfer of a known amount of sodium borohydride.

\subsection{The Reaction of Indium Acetate and Red Phosphorus: The Formation of Indium Oxide (Experiment 1 and Experiment 2)}

Experiments were carried out aiming at synthesizing indium phosphide from red phosphorus and indium acetate. However, all experiments failed to produce indium phosphide. Crystalline indium oxide $\left(\mathrm{In}_{2} \mathrm{O}_{3}\right)$ nanoparticles were among a variety of products observed and were the only product that could be characterized unambiguously by analysing the corresponding electron diffraction pattern. In both experiment 1 and 2, crystalline indium oxide nanoparticles were observed to be the only crystalline product. 
In experiment 1, a coordinating solvent, trioctylphosphine oxide was used both as a solvent and as a surfactant. As may be seen figure 4.1a, the nanoparticles were in an aggregated form. The aggregates were nearly spherical and have an average diameter of $16.2 \pm 5.4 \mathrm{~nm}$. No high resolution images of aggregates were obtained due to the presence of impurities. An SAED pattern analysis (figure 4.1b) showed that the nanoparticles were composed of indium oxide of formula $\operatorname{In}_{2} \mathrm{O}_{3}$.

In experiment 2, a non coordinating solvent, tetracosane $\left(\mathrm{CH}_{3}\left(\mathrm{CH}_{2}\right)_{22} \mathrm{CH}_{3}\right)$ was used as a solvent and trioctylphosphine oxide was used as a surfactant. Tetracosane is a straight chain heavy alkane which does not have a coordinating functional group and therefore is not expected to act as a surfactant such as trioctylphosphine oxide. The product obtained from the experiment consisted of aggregated crystalline indium oxide nanoparticles. The aggregates were constituted of crystalline nanoparticles of $\leq 10 \mathrm{~nm}$ randomly connected to each other as shown in figure 4.1c. The SAED pattern of the nanoparticles was the same as present in figure 4.1b. The ring pattern indicated that the nanoparticles that formed the aggregates were polycrystalline in nature. 
Chapter 4
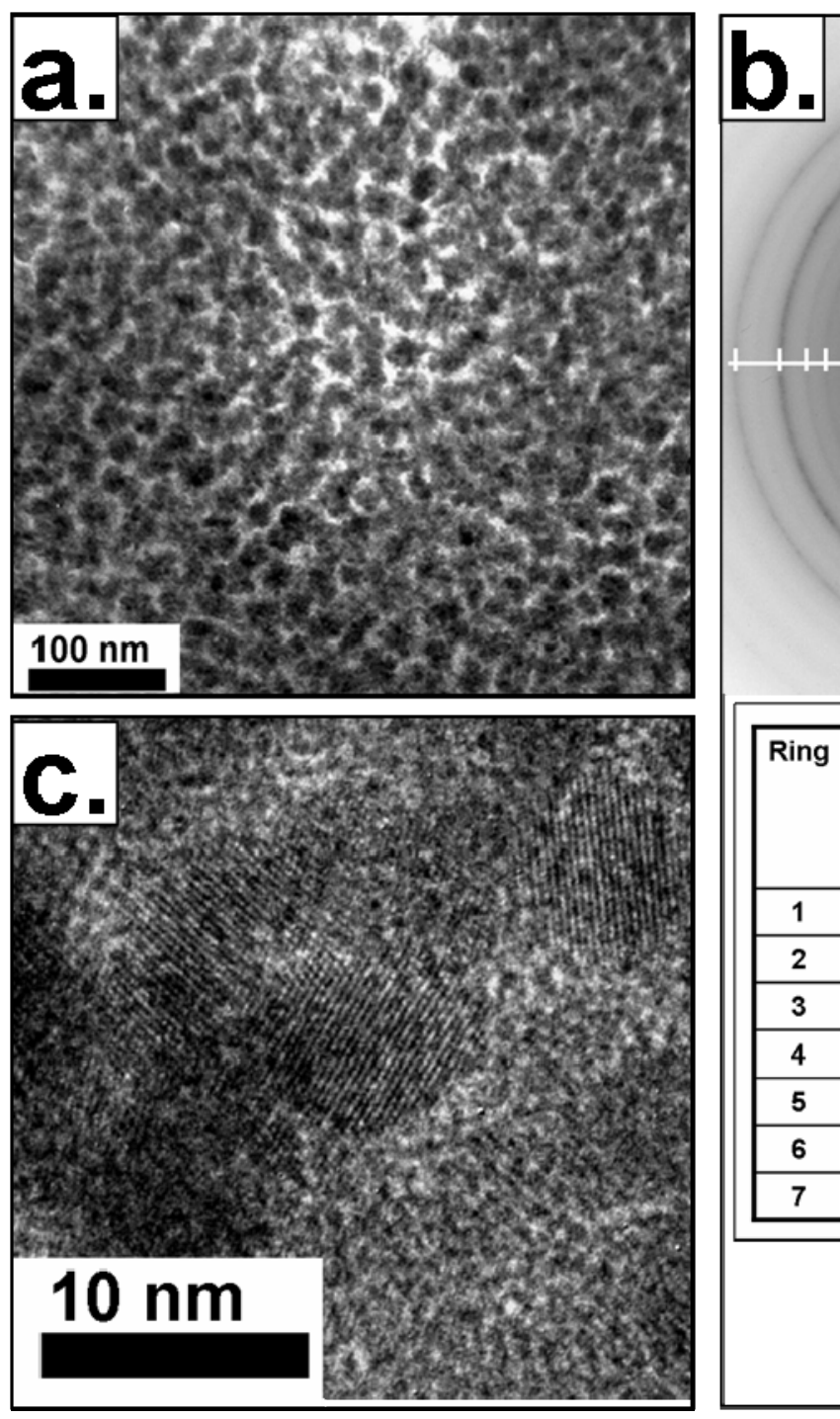

\begin{tabular}{||c|c|c|c|}
\hline Ring & $\begin{array}{c}\text { Miller Indexes } \\
\text { hkl }\end{array}$ & $\begin{array}{c}\text { d-spacing } \\
\text { A } \\
\text { calculated }\end{array}$ & $\begin{array}{c}\text { d-spacing } \\
\text { A } \\
\text { reference }\end{array}$ \\
\hline 1 & 211 & 4.15 & 4.13 \\
\hline 2 & 222 & 2.90 & 2.92 \\
\hline 3 & 400 & 2.54 & 2.52 \\
\hline 4 & 332 & 2.15 & 2.15 \\
\hline 5 & 422 & 2.00 & 2.06 \\
\hline 6 & 440 & 1.81 & 1.78 \\
\hline 7 & 622 & 1.53 & 1.52 \\
\hline
\end{tabular}

Figure 4.1 (a) a TEM micrograph of indium oxide nanoparticles aggregates obtained in experiment 1 (b) Indexed SAED pattern of indium oxide aggregates (compared to reference 8) and (c) a HRTEM image of crystalline indium oxide nanoparticles obtained in experiment 2 .

Experiments 1 and 2 showed that no indium phosphide was formed by reacting indium acetate and red phosphorus under these synthetic conditions. The mechanism of which crystalline indium oxide nanoparticles formed from indium acetate was discussed in the following paragraph. No residual red phosphorus was observed in the isolated product. From a controlled experiment it was found that red phosphorus could interact with trioctylphosphine oxide at high temperature to form a colourless liquid compound. The colour of red phosphorus in general did not reappear after the liquid was cooled to room temperature. The identity of the liquid compound is unclear. 


\section{On The Formation of Indium Oxide from Indium Acetate}

The observation of crystalline indium oxide nanoparticles was later found to be consistent with a literature report by Peng's group. ${ }^{8}$ The group synthesized crystalline indium oxide nanoparticles from indium acetate, tetradecanoic acid (myristic acid) in octadecene. At high temperature indium myristate $\left(\operatorname{In}(\text { myristate })_{3}\right)$ was formed with the release of acetic acid. Indium myristate was isolated and heated directly to form indium oxide. The group argued that no pyrolysis of the compound actually occurred based on IR absorption spectrometry. They found that it was difficult to isolate a dry indium myristate precursor and the tiny amount of water $\sim 1 \mathrm{mg}$ present then hydrolysized the acid precursor at high temperature to form crystalline indium oxide nanoparticles. Although different solvents were used in experiment 1 and 2, the solvent did not play the most critical role. The critical reaction was the hydrolysis of indium myristate at high temperature. In experiments 1 and 2 the possibility of the presence of trace amount of water could not be excluded since no measurement was carried out on the amount of water present. Another possible source of water is via the condensation of myristic acid to form tetradecanoic anhydride releasing water as a by-product. The water generated could then hydrolyse indium myristate to form indium oxide. 


\subsection{The Reaction of Indium Trichloride and Red Phosphorus in Trioctylphosphine Oxide /Methyl- Myristate Mixture (Experiment 3)}

An experiment was carried out reacting red phosphorus and indium trichloride in methyl-myristate and trioctylphosphine oxide was used as a surfactant. No crystalline indium oxide nanoparticles were obtained in this experiment. Instead, amorphous spherical nanoparticles (figure 4.2) with an average diameter of $5.9 \pm 0.8 \mathrm{~nm}$ were obtained.

An EDS analysis showed that indium, oxygen, and phosphorus were present as main components. However, due to trioctylphosphine oxide also contributed to the overall phosphorus and oxygen contains, no accurate ratios of indium to phosphorus or indium to oxygen could be calculated. Due to the absence of diffraction pattern, the nanoparticles could not be further characterized.

The nanoparticles obtained could either be indium metal, amorphous indium oxide or amorphous indium phosphide. Indium metal nanoparticles may be expected to melt under electron beam due to its low melting point and would therefore appear amorphous under TEM conditions. In order to avoid oxide formation and to be able to calculate a accurate indium to phosphorus ratio, trioctylamine was used both as a solvent and a surfactant in experiments that follow. Also a reducing agent sodium borohydride was also added to the reaction in order to "activate" the reaction. The reasons the reducing agent were chosen were discussed in section 4.5. 

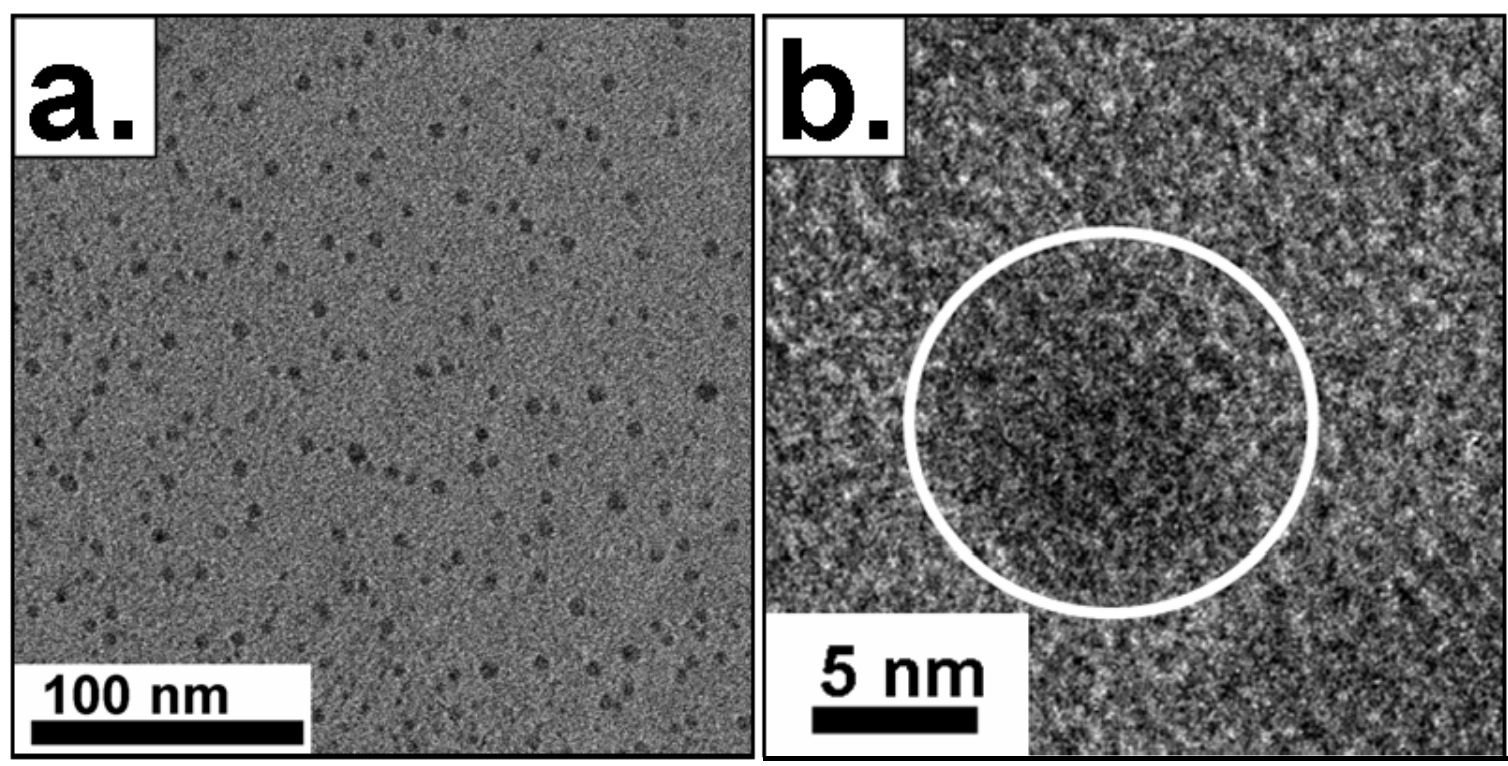

Figure 4.2 (a) A TEM image of amorphous nanoparticles obtained from experiment 2 and (b) a HRTEM image showed the amorphous nature of a nanoparticle.

\subsection{Reactions of Indium Trichloride and Red Phosphorus and Sodium Borohydride (Experiment 4)}

An experiment was carried out injecting sodium borohydride into a mixture of indium trichloride and red phosphorus. A black solid was isolated after purification. X-ray diffraction analysis showed the solid contained both crystalline indium metal and indium phosphide. No signals due to indium oxide were observed in the diffractogram. To remove indium metal, the solid was washed with diluted hydrochloric acid in methanol. As may be seen in figure 4.30, the main crystalline species present after acid treatment was face centre cubic indium phosphide. ${ }^{2}$ Three peaks close in position were observed below a $2 \theta$ value of $25^{\circ}$ were due to an impurity that could not be removed by the hydrochloric acid wash. 


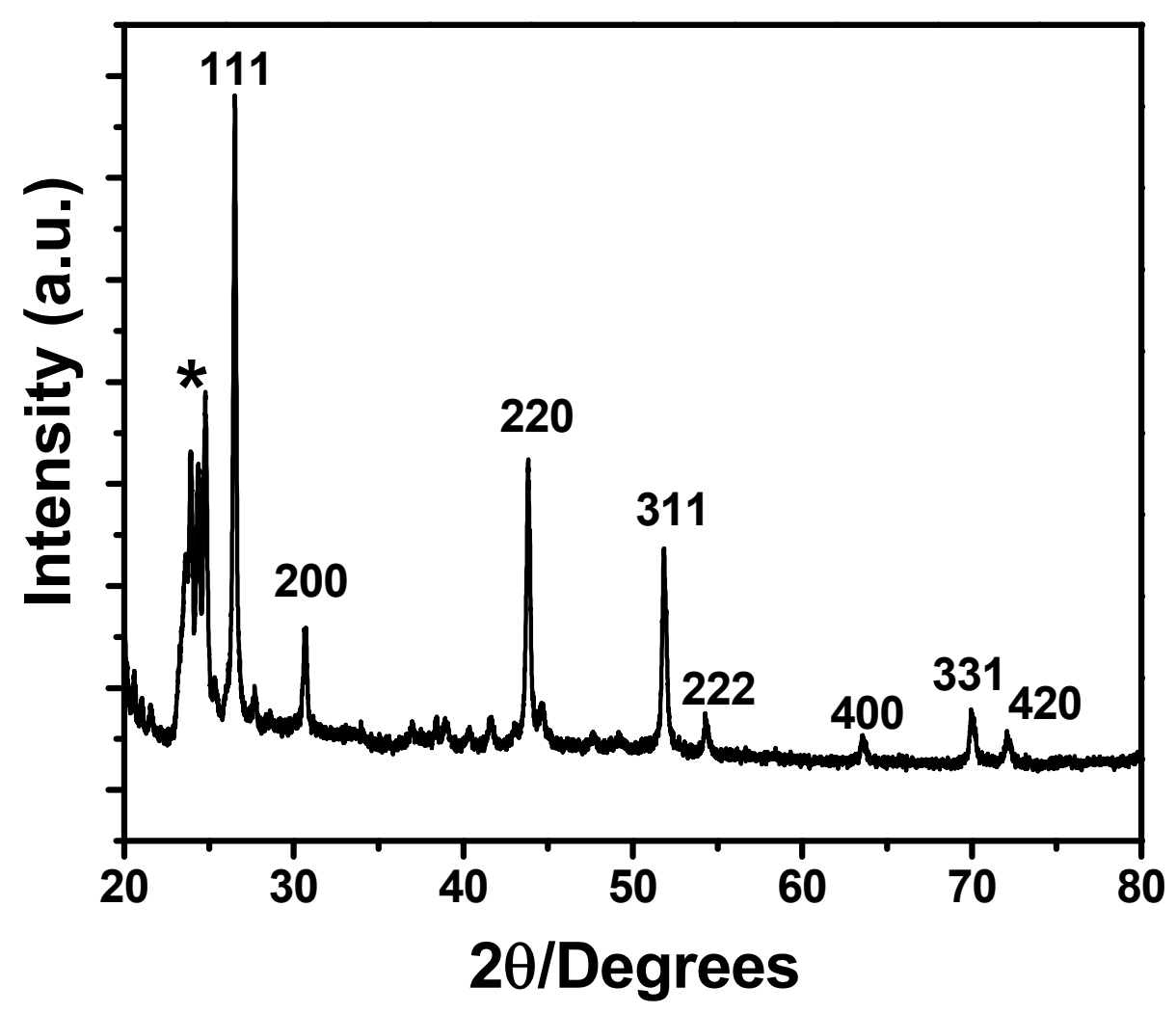

Figure 4.30 XRD pattern of a black solid isolated after diluted hydrochloric acid wash. The pattern corresponds to face centred cubic indium phosphide. A small amount of indium metal remained. The three peaks observed before $2 \theta$ of $25^{\circ}$ were due to an unknown impurity.

An FESEM study showed that the black solid consisted of nanowires and "chunky" solids. The nanowires were later shown to be indium phosphide by EDS studies and by HRTEM studies described in the next section. The chunky solid was composed of dominantly phosphorus from an EDS study on the sample (not shown).

To purify the nanowires from residue phosphorus, the solid was divided into three equal portions. One was treated with nitric acid, another with potassium hydroxide. The third part was sonicated in chloroform, allowed to settle and the supernatant separated from the precipitate. Figure 4.31 a,b,d,e are SEM images in backscattering mode and all four are of the same scale. The images were taken in backscattering mode because different species in the sample may exhibit different level of contrasts and would therefore allow direct visual recognition of different species. The ability to recognize different species based on image contrast was helpful when comparing images taken at low magnifications. 
In the images, big pieces of phosphorus exhibit moderate contrast while indium phosphide nanowires exhibit lighter contrast. It could be concluded that the acid and base treatment did not work in terms of removing big pieces of phosphorus from indium phosphide. Indium phosphide nanowires became scarce in both the acid and base treated samples.

Sonication-precipitation approach worked to a degree to remove a certain amount of red phosphorus. The supernatant contained a mixture of indium phosphide nanowires and pieces of phosphorus that were at least ten times smaller than those observed in the precipitate. Pieces of phosphorus observed in the precipitate were up to $10 \mu \mathrm{m}$ in diameter while that in the supernatant $\leq 1 \mu \mathrm{m}$. The supernatant was enriched with indium phosphide nanowires (figure 4.31c and d) while the precipitate with relatively large pieces of phosphorus. However, the method has disadvantages including the loss of indium phosphide nanowires in the precipitate and that small pieces of phosphorus remained in the indium phosphide nanowires enriched supernatant. Theoretically, the method could be repeated in many cycles to isolate purer and purer indium phosphide nanowires. 


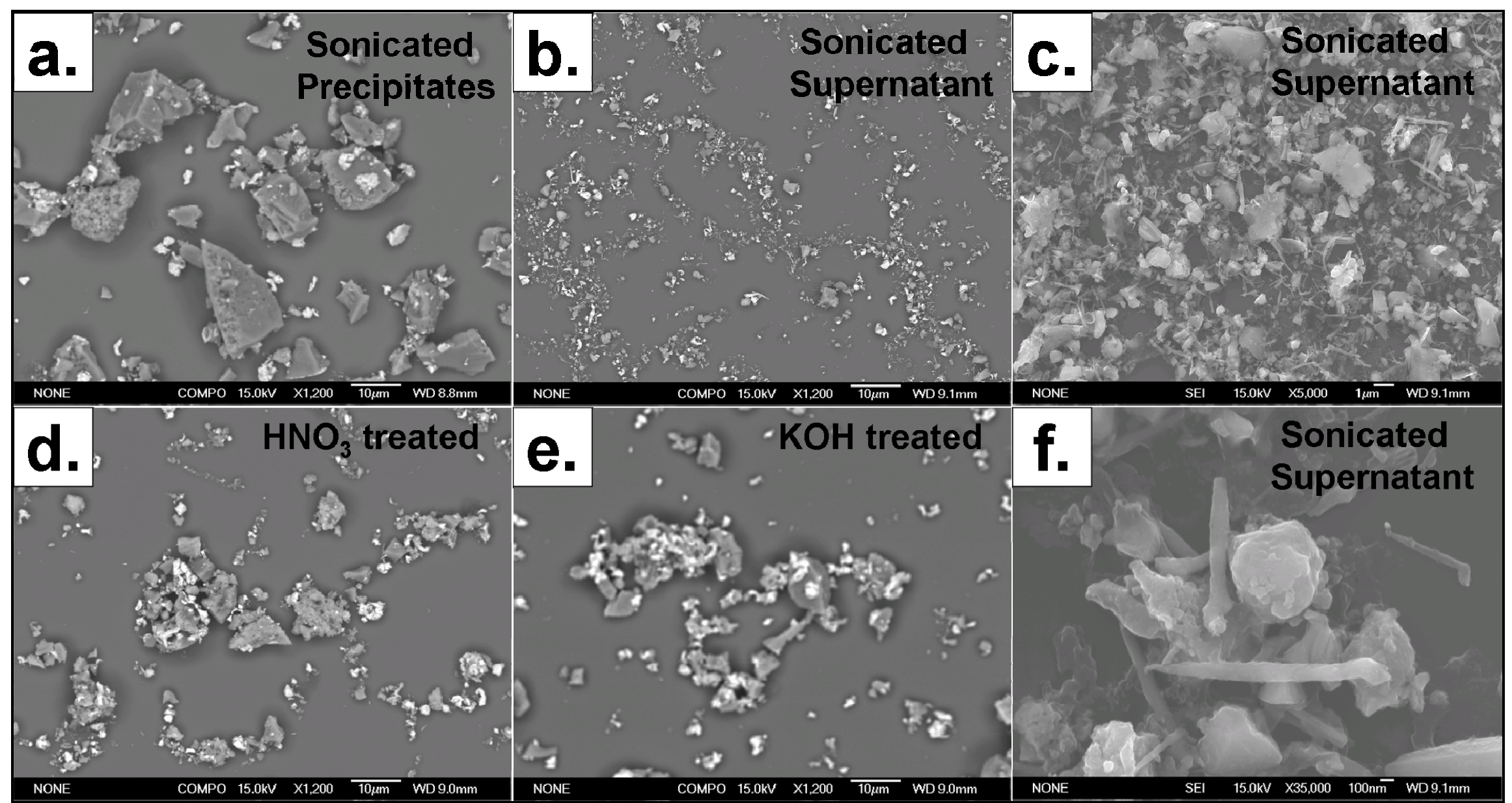

Figure 4.31. FESEM images. (a) residue phosphorus particles of $10 \mu \mathrm{m}$ in size. (b) indium phosphide nanowires and residue phosphorus $\sim 1 \mu \mathrm{m}$ in size. (c) $b$ in larger magnification. (d) residue phosphorus particles $\sim 1-10 \mu \mathrm{m}$. (e) residue phosphorus particles 1-10 $\mu \mathrm{m}$. (f) a close look at indium phosphide nanowires shown in c. Please note that $\mathrm{c}$ and $\mathrm{f}$ were images taken in SEI mode and a,b,d,e images taken in backscattering mode. 


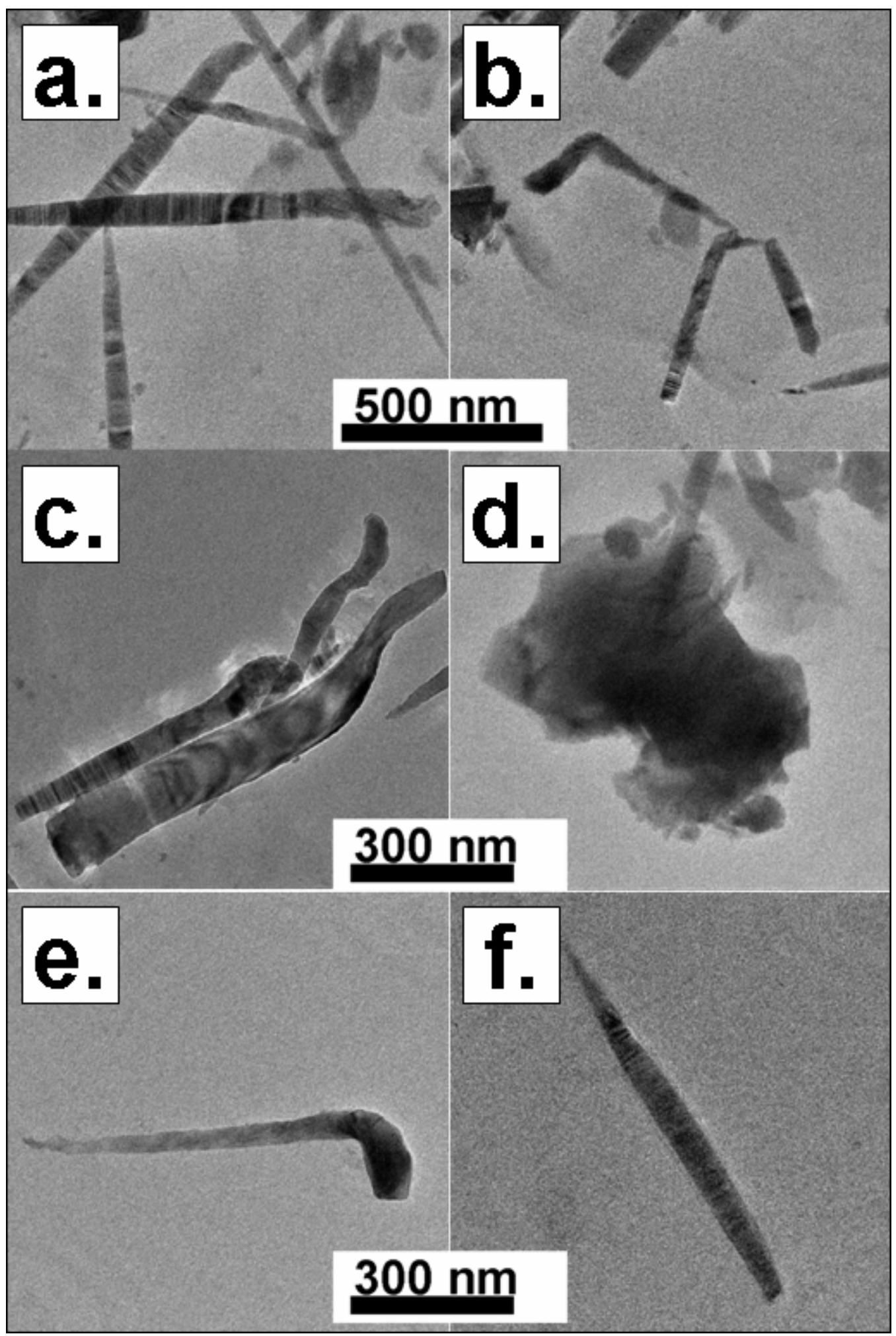

Figure 4.32 TEM images of indium phosphide nanowires after hydrochloric acid wash. Three types of nanowires observed: straight nanowires $(a, f)$, nanowires with twists (c) and hockey-stick shape nanowires (e) and nanoparticles (d). 
Chapter 4

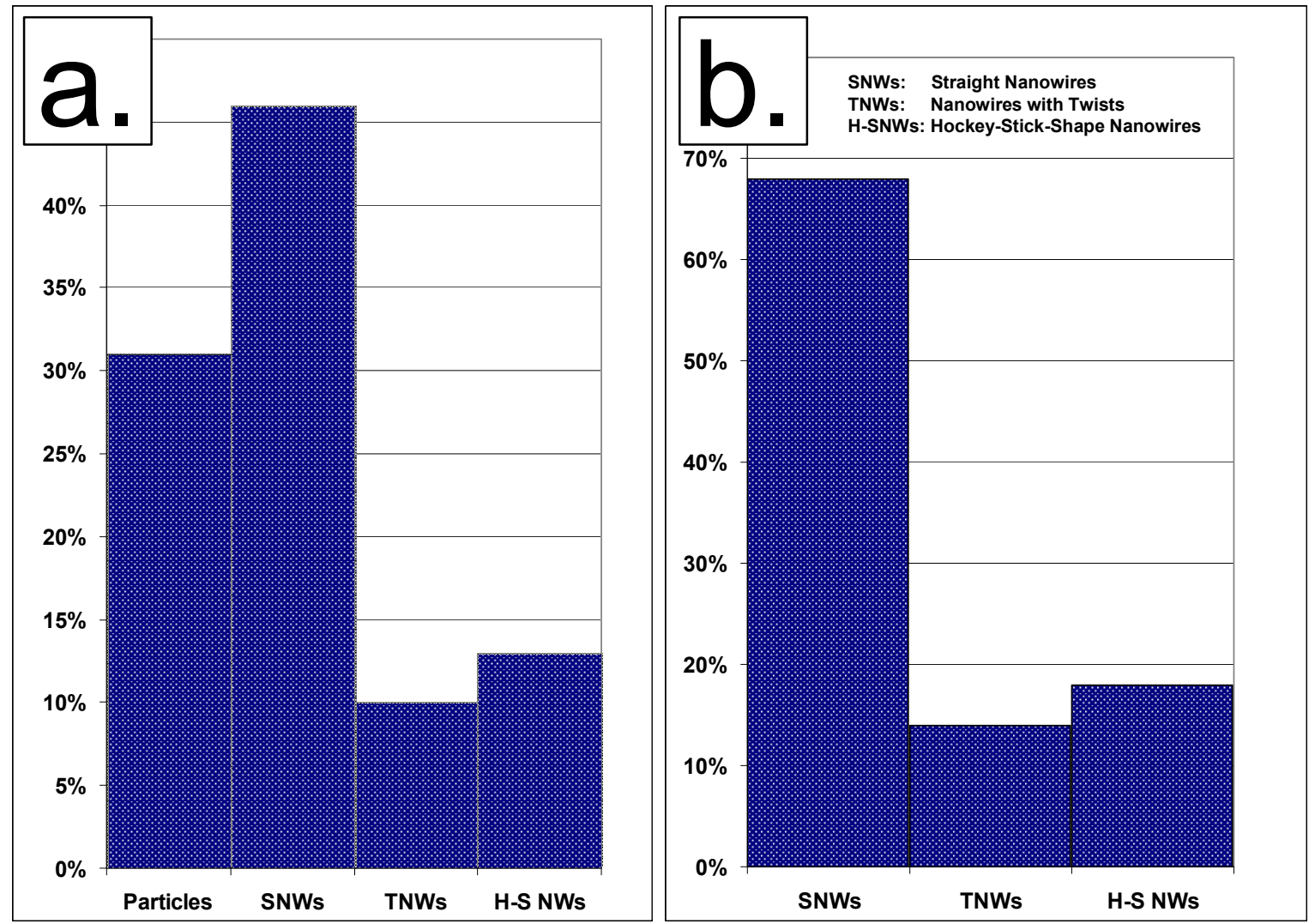

Figure 4.33 Histogram of (a) nanostructures of different shapes and (b) amongst nanowires.

Samples subjected to TEM analysis were purified from dilute hydrochloric acid. Of all nanostructures observed, approximately $31 \%$ was found to be particles and the remaining 69\% nanowires (figure 4.33a). Three types of nanowires were observed. Of all nanowires, 68\% was straight (figure 4.32 a and f), 18\% was of hockey-stick-shape (figure $4.32 \mathrm{~b}$ and e) and the remaining 14\% contained twists (figure $4.32 \mathrm{c}$ ). For hockey-stick-shape nanowires, the "stickheads" always have a diameter larger than that of the "handle". The diameters and lengths and twist angles of indium phosphide nanowires produced were summarized in table 4.34 .

Table 4.34 Diameters and lengths of three types of nanowires.

\begin{tabular}{|c|c|c|c|c|}
\hline \multicolumn{2}{|c|}{ Wire type } & Diameter/ nm & Length $/ \mathbf{n m}$ & Angle/ \\
\hline \multicolumn{2}{|c|}{ Straight } & $61 \pm 19$ & $730 \pm 272$ & N/A \\
\hline \multicolumn{2}{|c|}{ Twist } & $75 \pm 41$ & $694 \pm 210$ & $141 \pm 8^{\circ}$ \\
\hline \multirow{2}{*}{$\begin{array}{c}\text { Hockey- } \\
\text { stick }\end{array}$} & "Handle" & $52 \pm 22$ & $727 \pm 207$ & \multirow{2}{*}{$100 \pm 5^{\circ}$} \\
\cline { 2 - 4 } & $\begin{array}{c}\text { "Stick- } \\
\text { Head" }\end{array}$ & $83 \pm 21$ & $209 \pm 68$ & \\
\hline
\end{tabular}



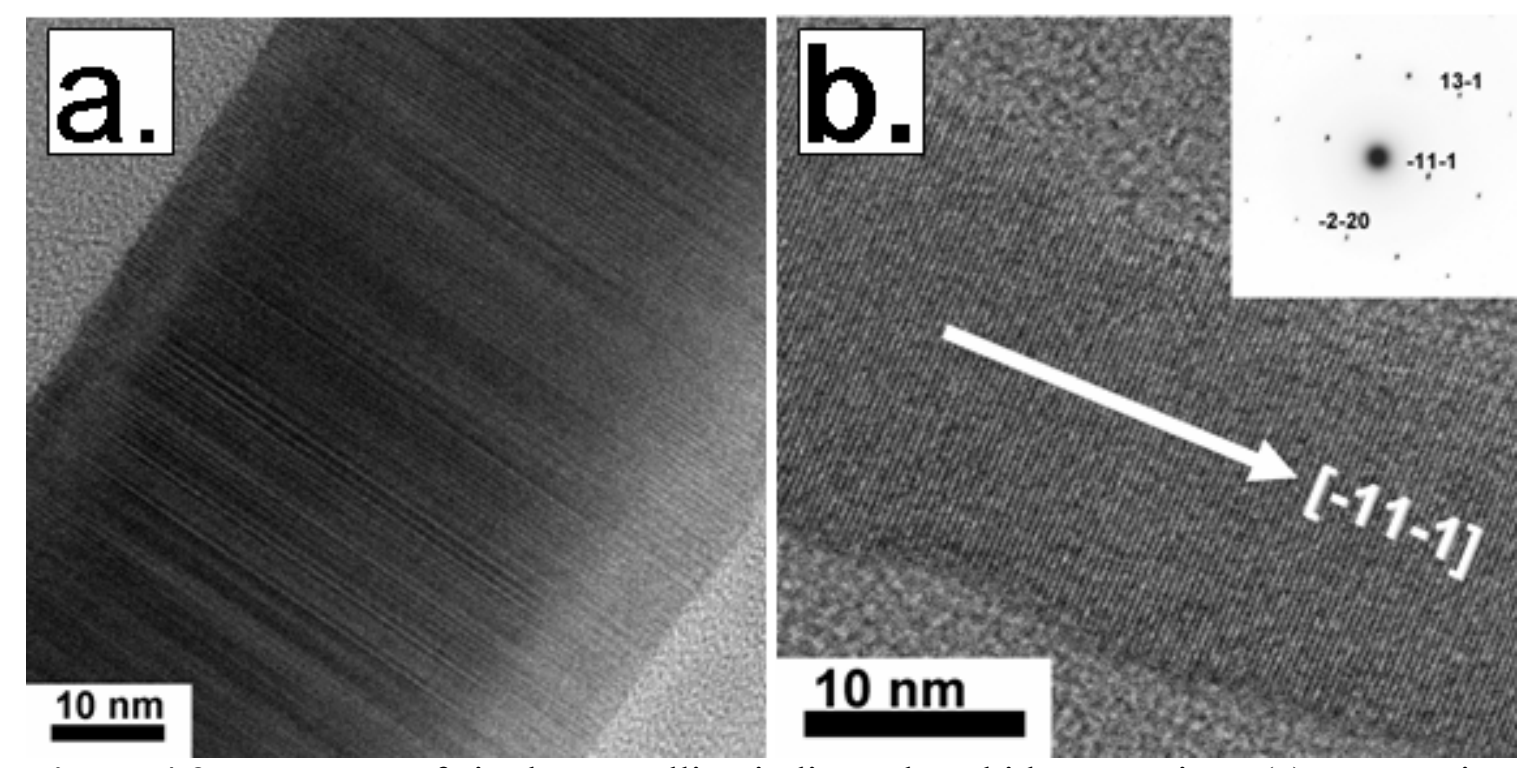

Figure 4.35 HRTEM of single crystalline indium phosphide nanowires (a) a nanowire with a diameter of $\sim 40 \mathrm{~nm}$ in size and (b) a nanowire of $\sim 12 \mathrm{~nm}$ in size growing in the [111] direction.

HRTEM studies revealed that most nanowires were highly crystalline. Figure 4.35 showed two single crystalline indium phosphide nanowires in details. Lattice fringes could be resolved and was observed to span across nanowires. The nanowires are of different thickness and diameters as evidenced by the different level of contrast observed in the HRTEM images. The nanowire in figure 4.35a was thicker than that in figure $4.35 \mathrm{~b}$. The growth direction of the indium phosphide nanowire (figure $4.35 \mathrm{~b}$ ) below was determined to be [111]. From literature, the [111] is the most common growth direction for cubic indium phosphide nanowires. ${ }^{10-12}$

This experiment showed that indium phosphide could be synthesized in the form of nanowires from indium trichloride and red phosphorus in the presence of sodium borohydride. The reaction produced indium phosphide nanowires in a low yield with the purified product still contained a significant amount of un-reacted phosphorus residues. Attempts to remove the red phosphorus residue by treating the sample with nitric acid and potassium hydroxide were unsuccessful. The purity of indium phosphide nanowires could be significantly enhanced by sonication-precipitation method. Indium phosphide nanowires and phosphorus residue ten times smaller in size were isolated from pieces of phosphorus residue some 10 microns in size. The reaction mechanism was discussed in section 4.5. Evidence of growth mechanism were obtained by studying the experiment that follows this section. 


\section{Optical Properties of Indium Phosphide Nanowires}

The sample suspended in chloroform was subjected to UV-VIS absorption measurement at room temperature. As may be seen in figure 4.36a, the sample absorbed across the entire visible spectrum with an emission maxima at $\sim 430 \mathrm{~nm}$. The absorption was broad and a sharp decrease in absorption after $700 \mathrm{~nm}$ was noticed
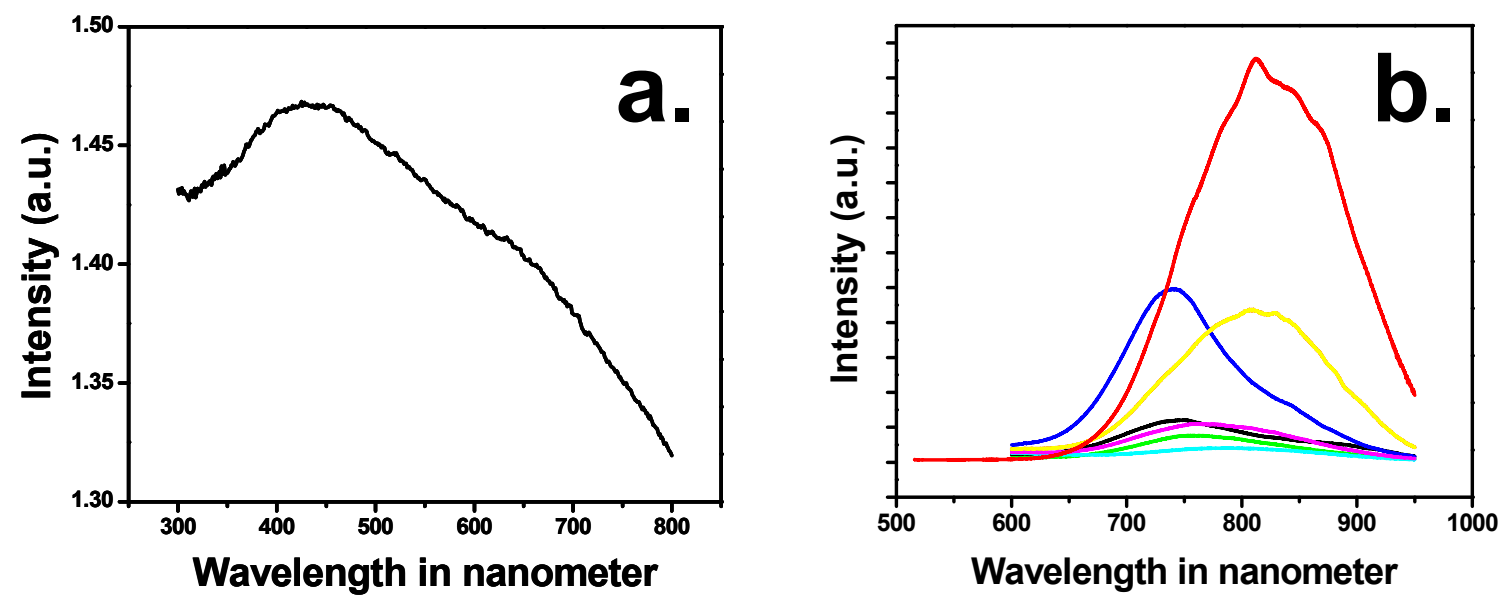

Figure 4.36 UV-VIS absorption and photoluminescence of indium phosphide nanowires. Spatial variations of both emission maxima and intensities were observed.

The sample dried on a piece of silicon was subjected to photoluminescence study at $293 \mathrm{~K}$. An $\mathrm{Ar}^{+}$ion laser with a wavelength of $514 \mathrm{~nm}$ was used to excite the sample. Figure $4.5 \mathrm{~b}$ showed seven photoluminescence spectra taken on seven spatially different locations. Emissions were all broad and emission maxima ranged from $720 \mathrm{~nm}$ to 820 nm. A control experiment with red phosphorus confirmed that red phosphorus did not exhibit photoluminescence in the same region under illumination of the $\mathrm{He}^{+}$laser. Therefore the photoluminescence observed must be due to indium phosphide nanowires present in the sample consisting of indium phosphide nanowires and pieces of phosphorus. 


\section{Comments on the Optical Properties of Indium Phosphide Nanowires Synthesized in Trioctylamine}

Indium phosphide nanowires obtained have diameters larger than the bulk Bohr radius of indium phosphide $(\sim 20 \mathrm{~nm})$ therefore no quantum confinement effect was expected. However, the blue shift in photoluminescence emission maxima has been previously observed in bulk indium phosphide nanowires. The origin of the shift is unclear. To further complicate the situation, nanowires grown in different axial direction could exhibit different emission maxima. A report by Dileep and $\mathrm{Fu}$ showed that indium phosphide nanowires grown in the [111] direction could theoretically have a band gap of $0.1 \mathrm{eV}$ higher than those grown in the [110] direction. ${ }^{12}$ It is impossible to prove that all indium phosphide nanowires prepared have grown in the [111] direction as the one determined in figure $4.36 \mathrm{~b}$. The photoluminescence experiment probed an ensemble of nanowires which could have different diameters, lengths and growth directions. The emission observed is a collection of all signals originating from the ensemble. This was particularly manifested in the observation of spatial dependence of photoluminescence spectra.

\subsection{Reactions of Indium Trichloride and Red Phosphorus and Sodium Borohydride (Experiment 5)}

An experiment was carried out injecting sodium borohydride into indium trichloride prior to the addition of red phosphorus. The colourless solution of indium trichloride in trioctylamine reacted with sodium borohydride to form indium metal species evidenced by the appearance of a greyish black solution. Having reacted with red phosphorus, the reacted mixture was purified and a black solid was isolated. The solid was studied in the FESEM.

As may be seen in figure 4.40 the sample consisted of nanowires, spherical nanoparticles and aggregated flakes-like nanostructures. The nanowires have diameters ranging from $0.1 \mu \mathrm{m}$ to $3.3 \mu \mathrm{m}$ and lengths 2.7 to $12 \mu \mathrm{m}$. Due to that multiple products present as a mixture no statistically meaningful analysis in diameter and length 
distribution could be attained from analyzing the FESEM images. However, a ubiquitous observation of long wire with a spherical tip is the most noticeable.

The frequent observation of nanowires with larger spherical nanoparticle attached to their tips on one end indicated the nanowires were grown via solution-liquid-solid (SLS) growth. The spherical nanoparticles at the tips and the nanowires existed as singular units. The spherical nanoparticles were always larger in diameter than the main section of the nanowires they attached to. Diameter ratios (diameter of spherical nanoparticles/diameter of nanowire) ranging from 1.5 to 3.8 were observed. Longer nanowires have larger diameter ratios when compared to shorter nanowires. Figure 4.41 shows an indium phosphide nanowire extending from a large spherical tip and gradually tapering into a sharp end. A bundle of nanowires with diameter 4.5 times smaller was also observed.

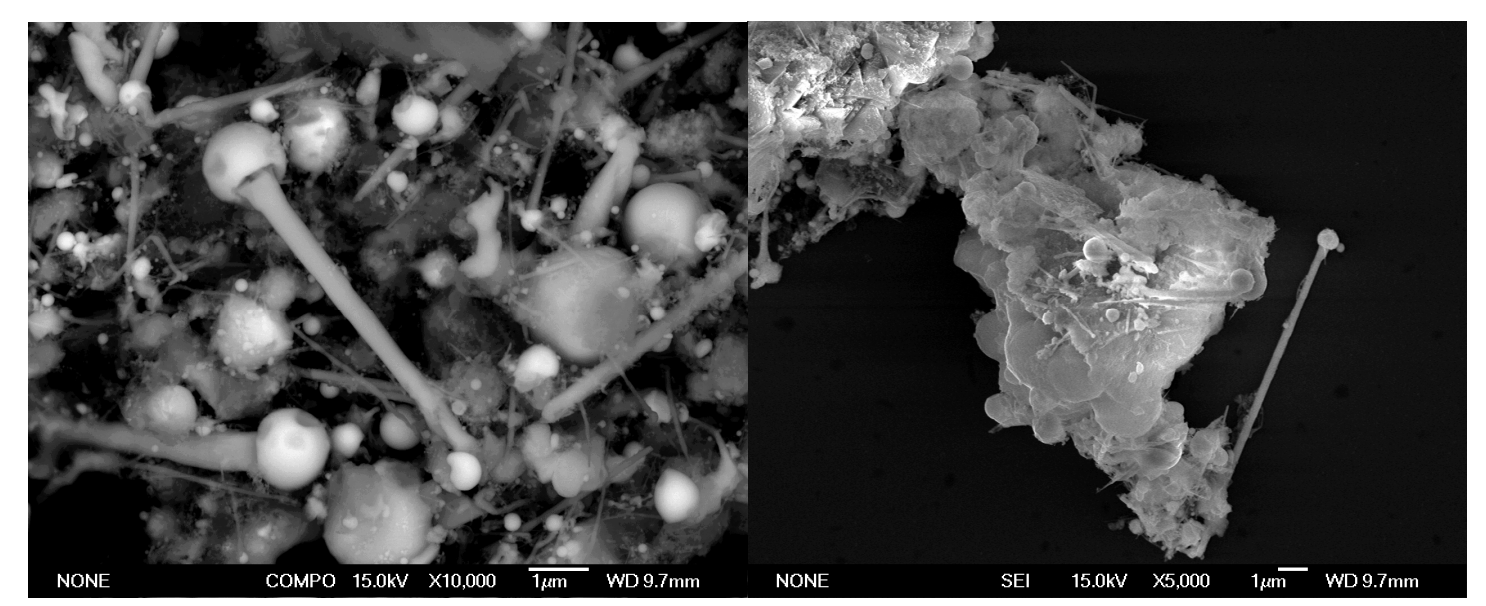

Figure 4.40 FESEM images of indium phosphide nanowires. Nanowires with spherical nanoparticles attached to one end of the nanowires were common. Structures other than nanowires were also observed. 


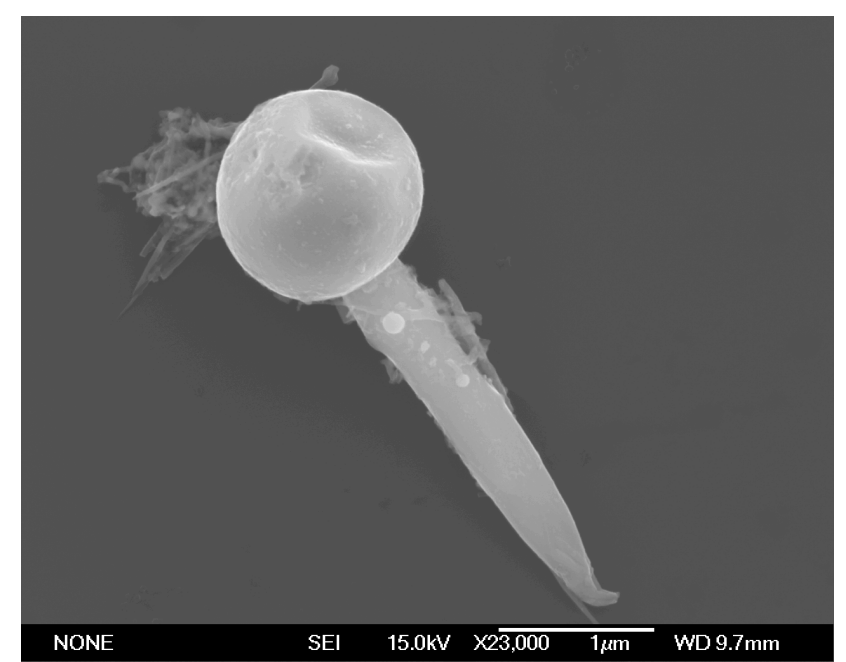

Figure 4.41 A closer examination of an indium phosphide nanowire with a spherical cap. Note the presence of some particles and bundles of much smaller nanowires near the cap.

\section{Solution-Liquid-Solid (SLS) Growth of Nanowires}

Detailed descriptions of SLS growth and how it is related to VLS growth is given in section 1.3 in chapter 1 . The following paragraph is presented only as a recapitulation of the concept. Solution-Liquid-Solid growth was first proposed by Trentler and cowokers in 1996 as an analogy of VLS growth. ${ }^{4}$ Indium phosphide nanowires were observed to form in a refluxing solution of organometallic indium compounds. Indium phosphide nanowires with spherical indium nanoparticles attached to their tips were observed. This is very similar to the characteristic appearance of VLS grown nanowires. VLS and SLS growth differ from each other in that in SLS growth molten metal droplets are formed prior to alloy formation. As may be seen in figure 4.42 a molten metal droplet forms when heated at a temperature higher than its melting point. The liquid droplet dissolves reactant species and forms a liquid alloy droplet. Upon supersaturation, reactant species crystallizes out as minute nuclei. More reactant species then condense onto the nuclei and axial growth commences and prolonged growth leads to the formation of nanowires. 


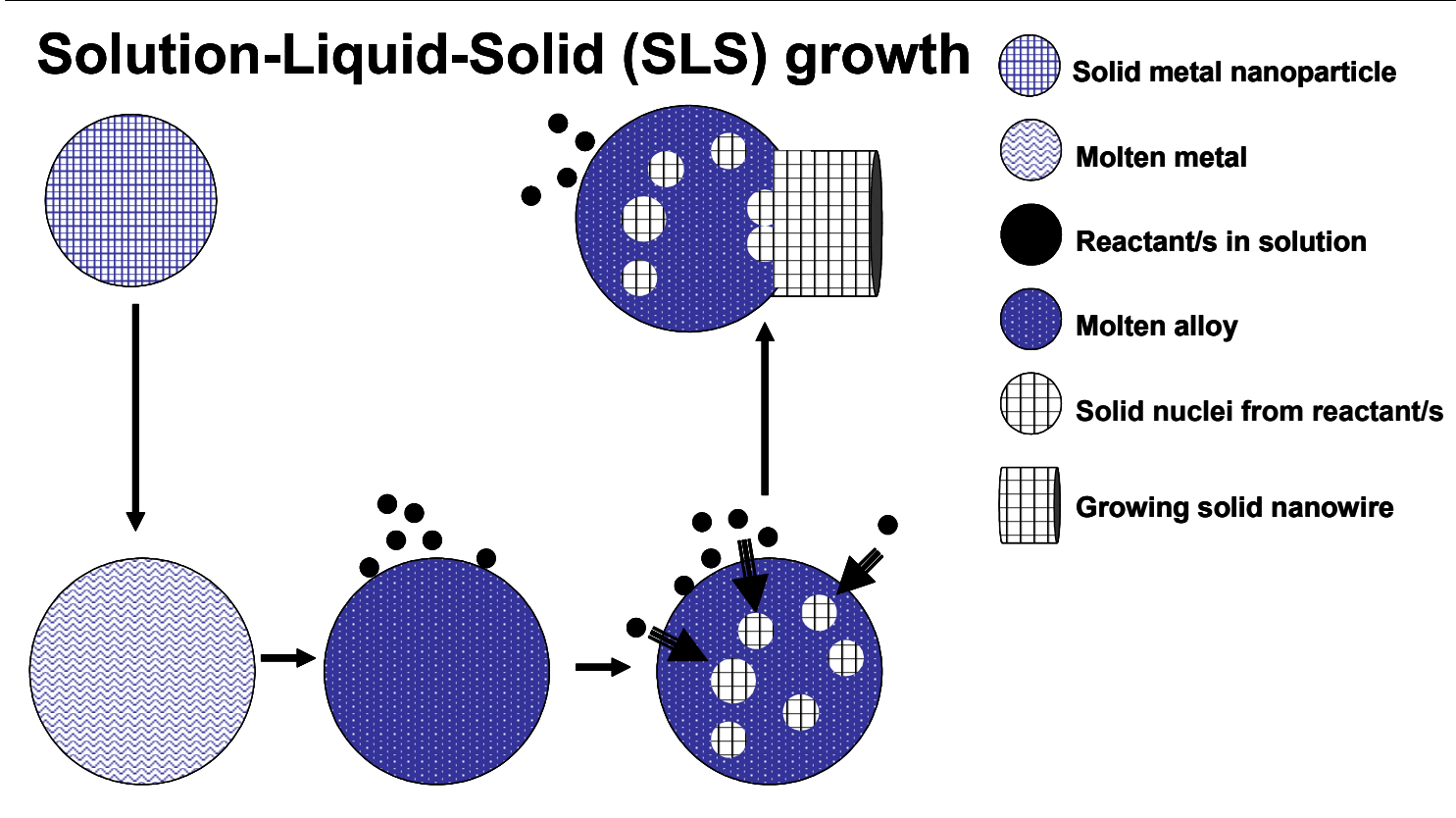

Figure 4.42 Schematic diagrams of SLS growth of a nanowire.

The nanowire shown in figure 4.41 was investigated with EDS mapping analysis. As shown in figure 4.43 , the nanowire was constituted of indium phosphide. The spherical tip was found to consist of mainly indium metal although a small amount of phosphorus was also observed. The tip may contain a certain amount of indium phosphide. However, the actual location of indium phosphide residue could not be determined exactly. The presence of a small amount of oxygen on the surface of the spherical tip suggested the surface indium may be oxidized. 
Chapter 4

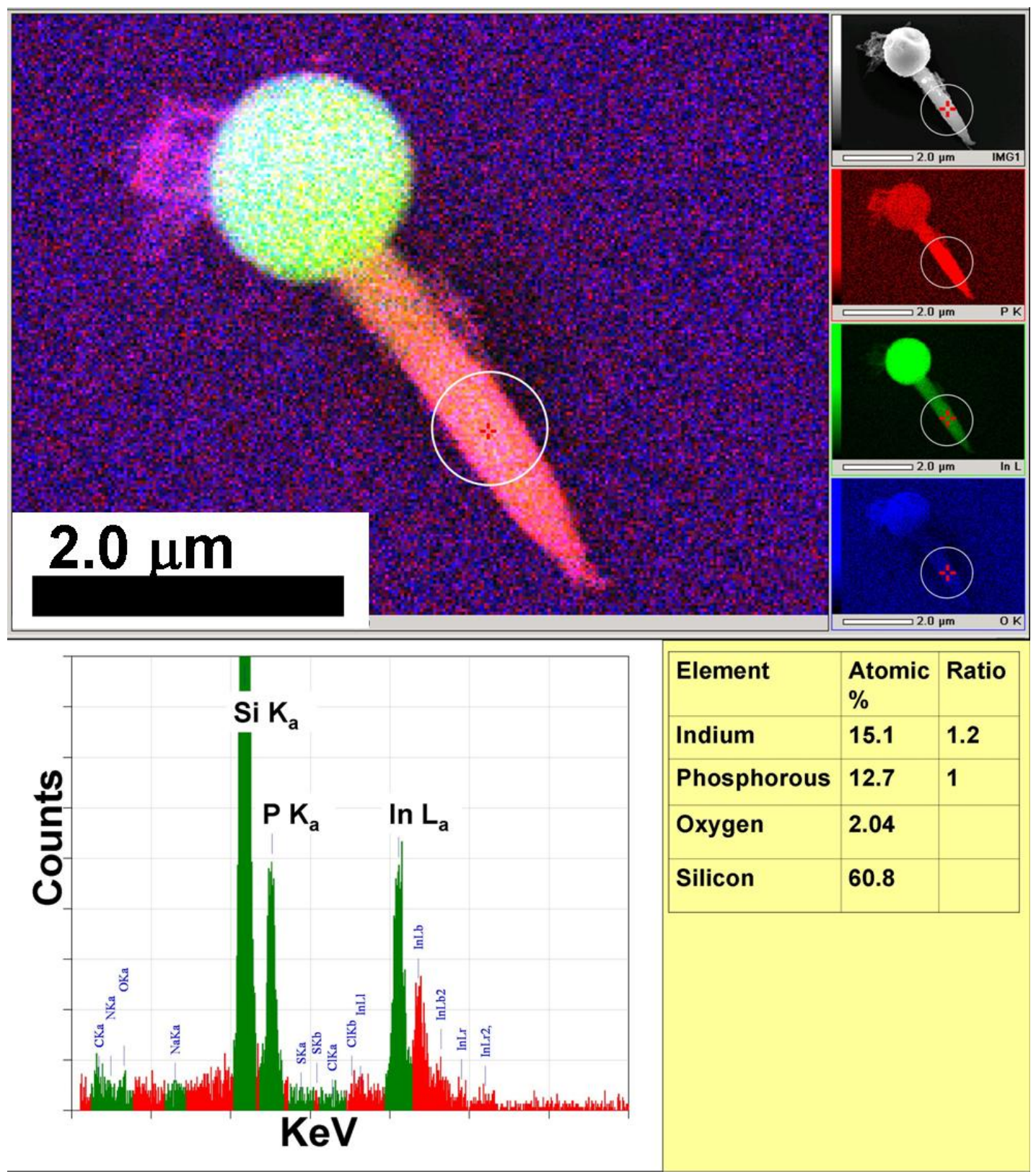

Figure 4.43 An EDS mapping of the indium phosphide nanowires as shown in figure 4.41. The nanowire (in pink colour) is constituted of indium phosphide and the spherical nanoparticle indium metal. 


\subsection{Discussion}

\section{Chemistry and The Role of Sodium Borohydride}

Crystalline indium phosphide nanoparticles have been previously prepared by Nann's group by reacting indium acetate with tris-trimethylsilylphosphine in methyl-myristate in the presence of tetradecanoic acid. ${ }^{13}$ In experiments 1 and 2 no crystalline indium phosphide was observed. By comparing the experiments to Nann's report, it was concluded red phosphorous did not react in a similar way to trimethylsilylphosphine. The reason behind could be that red phosphorous is not reactive enough or that the reaction pathway 1 (scheme 4.50) was not accessible. Therefore a new reaction pathway 2 (scheme 4.51) was proposed. To generate indium metal species, a hydride reduction was used. Indium trichloride was chosen to be reduced instead of indium acetate in order to avoid any oxide formation that would complicate up the system. Sodium borohydride was chosen because of its availability in solid form which could be suspended in trioctylamine after being sonicated for a short period of time. The byproducts of the reduction were expected to be sodium chloride which could be removed by water or alcohol wash and borane $\left(\mathrm{BH}_{3}\right)$ / diborane $\left(\mathrm{B}_{2} \mathrm{H}_{6}\right)$ gas would have escaped the system during the synthesis.

Scheme 4.50 Proposed reaction pathway for the synthesis of indium phosphide.

$\operatorname{In}\left(\mathrm{OCOCH}_{3}\right)_{3}+\mathbf{P}_{(\text {red })} \longrightarrow \longrightarrow$ InP ------------ Pathway 1

Scheme 4.51 Proposed reactions for the synthesis of indium phosphide.

$$
\begin{aligned}
& \mathrm{InCl}_{3}+3 \mathrm{NaBH}_{4} \longrightarrow \mathrm{In}+3 \mathrm{BH}_{3}+3 \mathrm{NaCl}+3 / 2 \mathrm{H}_{2} \\
& \mathrm{In}+\mathrm{P} \longrightarrow \mathrm{InP}
\end{aligned}
$$

\section{Growth Mechanism of Nanowires}

Indium phosphide nanowires were believed to grow via solution-liquid-solid (SLS) growth. For nanowires grown via SLS growth it is characteristic of them to appear in the form of a wire with a spherical cap similar to that shown in figure 4.42 . ${ }^{4,5 \mathrm{a}}$ 
EDS mapping indicated the nanowire section was made up of indium phosphide. The composition of spherical tip is more complicated. The tip from which the nanowire grew is likely to compose of indium/phosphorus alloy or even indium phosphide. The outer shell could be indium oxide formed in contact with air. The layer in between is likely to be composed of just indium metal. EDS mapping in the SEM did not show the depth of probing therefore the actual position in space where signals originated could not be determined.

\section{On The Formation of Three Different Nanowires}

It is unclear regarding the formation of three differently shaped nanowires in a one-pot synthesis. The twist and hockey-stick-shape nanowires formed probably due to the kinetics reasons including local concentration of indium and phosphorus nuclei and local temperature. There is no obvious evidence of any particle-size effect in determining the type of nanowires formed since the diameters of various types of nanowires were similar.

\section{The Effect of Reaction Sequences}

Comparing experiment 4 and 5 it is obvious that there exists an effect on reaction sequence. Smaller nanowires were obtained in experiment 4 and larger and longer nanowires were obtained in experiment 5 . In experiment 5, indium metal was formed prior to the addition of red phosphorus. Larger indium metal nanoparticles were expected since they could grow before the addition of red phosphorus. With larger indium metal nanoparticles, nanowires with larger diameter therefore formed. The lengths of nanowires formed in experiment 5 were also much longer when compared to that of produced in experiment 4. The difference may be due to the availability of indium supply. A more immediate supply of indium was expected in experiment 5 than that in experiment 4 since in experiment 5 indium metal was formed prior to the addition of red phosphorus. With a higher and more immediate supply of indium metal, indium phosphide may form at a higher rate and therefore lead to longer nanowires. 
Table 4.53 Selected experiments

\begin{tabular}{|c|c|c|c|c|c|c|}
\hline \multicolumn{7}{|c|}{ In(acetate) ${ }_{3}$ and $P$ (red) } \\
\hline Experimental & \multicolumn{2}{|c|}{$\begin{array}{c}\text { Temperature } \\
\left({ }^{\circ} \mathrm{C}\right)\end{array}$} & \multirow[t]{2}{*}{$\begin{array}{l}\text { Time } \\
\text { (hrs) }\end{array}$} & \multirow[t]{2}{*}{$\begin{array}{c}\text { Solvent/ } \\
\text { Surfactants }\end{array}$} & \multirow[t]{2}{*}{ Sequence } & \multirow[t]{2}{*}{ Products } \\
\hline & Injection & Growth & & & & \\
\hline 1 & 100 & $300-350$ & 6 & $\begin{array}{c}{ }^{1} \mathrm{TOPO} /{ }^{2} \mathrm{HDA} \\
{ }^{3} \mathrm{TDNA}\end{array}$ & $\begin{array}{l}\text { 1. In(acetate })_{3} \text { and } \mathrm{P} \text { powder were mixed. } \\
\text { 2. (1) was added to a solvent mixture at } 104{ }^{\circ} \mathrm{C} \\
\text { 3. (2) was heated at } 350{ }^{\circ} \mathrm{C} \text { for } 5.5 \text { hours. }\end{array}$ & $\begin{array}{l}\text { Aggregated } \\
\text { crystalline } \operatorname{In}_{2} \mathrm{O}_{3} \\
\text { nanoparticles }\end{array}$ \\
\hline 2 & 110 & 350 & 24 & $\begin{array}{l}\text { Tetracosane } \\
\text { TDNA }\end{array}$ & $\begin{array}{l}\text { 1. In(acetate })_{3} \text { and P powder were mixed. } \\
\text { 2. (1) was added to solvent mixture at } 110{ }^{\circ} \mathrm{C} \\
\text { 3. (2) was heated at } 350{ }^{\circ} \mathrm{C} \text { for } 23.5 \mathrm{hrs} \\
\end{array}$ & $\begin{array}{l}\text { Aggregated } \\
\text { crystalline } \mathrm{In}_{2} \mathrm{O}_{3} \\
\text { nanoparticles } \\
\end{array}$ \\
\hline \multicolumn{7}{|c|}{$\mathrm{InCl}_{3}, \mathrm{P}(\mathrm{red})$ and $\mathrm{NaBH}_{4}$} \\
\hline 3 & $\begin{array}{l}\text { Slow } \\
\text { addition } \\
250\end{array}$ & $250-300$ & 4 & $\begin{array}{c}\text { TOPO } \\
\text { Methyl myristate }\end{array}$ & $\begin{array}{l}\text { 1. } \mathrm{P} \text { and TOPO were mixed in methyl myristate. } \\
\text { 2. } \mathrm{InCl}_{3} \text { dissolved in TOPO was injected to }(1) \text {. } \\
\text { 3. The mixture from (2) was allowed to react for } \\
4 \text { hours. }\end{array}$ & $\begin{array}{l}\text { Amorphous } \\
\text { nanoparticles of } \\
\sim 6 \mathrm{~nm} \text { in size. } \\
\text { Chemical } \\
\text { composition } \\
\text { unclear. }\end{array}$ \\
\hline 4 & 250 & $260-330$ & 15 & ${ }^{4} \mathrm{TOA}$ & $\begin{array}{l}\text { 1. } \mathrm{InCl}_{3} \text { and } \mathrm{P} \text { were mixed in TOA. } \\
\text { 2. } \mathrm{NaBH}_{4} \text { suspended in TOA injected to }(1) . \\
\text { 3. The mixture from (2) was heated at } 250-330{ }^{\circ} \mathrm{C} \text { for } 15 \\
\text { hours. }\end{array}$ & $\begin{array}{l}\text { InP nanowires } \\
\text { In metals } \\
P \text { residues }\end{array}$ \\
\hline 5 & 260 & $290-340$ & 19 & TOA & $\begin{array}{l}\text { 1. } \mathrm{InCl}_{3} \text { was dissolved in TOA. } \\
\text { 2. } \mathrm{NaBH}_{4} \text { was suspended in TOA. } \\
\text { 3. The mixture from (2) was injected into (1). } \\
\text { 4. P suspended in TOA injected into (3). } \\
\text { 5. The mixture from (4) was heated for } 19 \mathrm{hrs} \text {. }\end{array}$ & $\begin{array}{l}\text { InP nanowires } \\
\text { In metals } \\
P \text { residues }\end{array}$ \\
\hline
\end{tabular}

${ }^{1}$ Trioctylphosphine oxide ${ }^{2}$ Hexadecylamine ${ }^{3}$ Tetradecanoic acid ${ }^{4}$ Trioctylamine 


\subsection{Conclusions}

A new method was successfully developed to synthesize highly crystalline indium phosphide nanowires in solution. Indium phosphide nanowires were produced via SLS growth from indium trichloride, red phosphorus and sodium borohydride. Sodium borohydride was essential for the successful preparation of indium phosphide nanowires. Three types of nanowires were observed: straight, twisted and hockey-stickshape. The nanowires were found to exhibit photoluminescence at $293 \mathrm{~K}$ under illumination of an $\mathrm{Ar}^{+}$ion laser. The emission maxima and intensity were found to vary spatially. The spatial variation was the result of non-uniform nanowire diameter and length distribution. All photoluminescence emission maxima are of higher energy with respect to that of bulk indium phosphide. The shifts have origins beyond quantum confinement effect because the nanowires are of dimensions larger than the Bohr radius of indium phosphide which is $\sim 20 \mathrm{~nm}$. 


\subsection{Experimental}

\section{Synthesis of Indium Phosphide Nanowires in Trioctylamine (Experiment 4)}

\section{Solution 1.}

A solution mixture containing $0.357 \mathrm{~g}$ in indium trichloride and $0.0487 \mathrm{~g}$ of red phosphorus and $10 \mathrm{~mL}$ to trioctylamine was heated to $190{ }^{\circ} \mathrm{C}$ under vacuum for one hour. The solution was then further heated to $250{ }^{\circ} \mathrm{C}$ under nitrogen.

\section{Solution2}

A suspension consisted of $0.2919 \mathrm{~g}$ of sodium borohydride and $4 \mathrm{~mL}$ trioctylamine was heated to $100{ }^{\circ} \mathrm{C}$ under vacuum for one hour. The solution was syringed under nitrogen before being injected.

\section{Injection and Growth}

All of solution 2 was injected into solution 1 at $250{ }^{\circ} \mathrm{C}$. A $15{ }^{\circ} \mathrm{C}$ temperature drop was observed 1 minute after the injection. The resulting solution mixture was heated in between $250-260{ }^{\circ} \mathrm{C}$ for 4 hours. The solution was cooled to $40{ }^{\circ} \mathrm{C}$ before subjected to vacuum for 40 minutes. The solution was then heated to a temperature between 300-340 ${ }^{\circ} \mathrm{C}$ for 15 hours under nitrogen.

\section{Purification}

\section{Liquid-Liquid Extraction}

After cooled to room temperature, the reacted mixture was transferred into a separating funnel and diluted with $40 \mathrm{~mL}$ of octane. Indium phosphide nanowires were extracted from the octane solution with $50 \mathrm{~mL}$ of $\mathrm{N}-\mathrm{N}$-dimethylforamide three times. All N-Ndimethylformamide fractions were combined and filtered over a filter paper in a Buchner funnel under suction. A layer of grey solid was collected on the filter paper. 


\section{Diluted Hydrochloric Acid Wash}

$0.20 \mathrm{~g}$ of the grey solid was treated with $30 \mathrm{~mL}$ of $1 \mathrm{M}$ aqueous hydrochloric acid at room temperature overnight. Greyish black solid less dense than water was collected on a filter paper and was washed with copious amount of water. The dried black solid was then dissolved in $3 \mathrm{~mL}$ of chloroform.

\section{Synthesis of Indium Phosphide Nanowires in Trioctylamine (Experiment 5)}

\section{Solution 1}

A solution containing $0.456 \mathrm{~g}$ in indium trichloride and $12 \mathrm{~mL}$ to trioctylamine was heated to $200{ }^{\circ} \mathrm{C}$ under vacuum for one hour. The solution was then further heated to $248{ }^{\circ} \mathrm{C}$ under nitrogen.

\section{Solution 2}

A suspension consisted of $0.3520 \mathrm{~g}$ of sodium borohydride and $4 \mathrm{~mL}$ trioctylamine was heated to $100{ }^{\circ} \mathrm{C}$ under vacuum for one hour. The suspension was later sonicated to form a fine suspension which was syringed under nitrogen before being injected.

\section{Solution 3}

A suspension consisted of $0.0085 \mathrm{~g}$ of red phosphorus powder and $5 \mathrm{~mL}$ of trioctylamine. The suspension was heated to a $100{ }^{\circ} \mathrm{C}$ under vacuum for 50 minutes. The suspension was syringed under nitrogen before being injected.

\section{Injection and Growth}

All of solution 2 was injected into solution 1 at $248{ }^{\circ} \mathrm{C}$. A $17{ }^{\circ} \mathrm{C}$ temperature drop was observed 1 minute after the injection. The resulting mixture was allowed to react at $\sim 250-260{ }^{\circ} \mathrm{C}$ for one hour to form a grayish black solution. All of solution 3 was injected into the grayish black solution at $261{ }^{\circ} \mathrm{C}$ and a jet black solution was formed. The jet black solution was heated at a temperature in between $250-340{ }^{\circ} \mathrm{C}$ for 19 hours. 


\section{Purification}

After cooled to room temperature, the reacted mixture was diluted with $30 \mathrm{~mL}$ of octane. The octane solution was centrifuged at $4000 \mathrm{rpm}$ for 5 minutes to isolate a grey to white solid precipitate. The precipitate containing indium phosphide nanowires was then suspended in $50 \mathrm{~mL}$ octane. Impurities were extracted from the octane solution with water three times in a separating funnel. Indium phosphide nanowires were isolated by centrifuging the octane fraction at $4000 \mathrm{rpm}$ for 2 minutes. The precipitate which contained indium phosphide nanowires were suspended in $10 \mathrm{~mL}$ of chloroform. 


\subsection{References}

1. Higgins, W. M.; Iseler, G. W.; Bliss, D.F.; Bryant, G.; Tassev, V.; Jafri, I.; Ware, R. M.; Carlson, D. J. J. Cryst. Growth 2001, 225, 225-230.

2. Naitoh, M.; Soga, T.; Jimbo, T.; Umeno, M. J. Cryst. Growth 1988, 93, 52-55.

3. Tsang, W. T.; Miller, R. C.; Capasso, F.; Bonner, W. A. Appl. Phys. Lett. 1982, 41, 467-469.

4. Trentler, T. J.; Hickman, K. M.; Goel, S. C.; Viano, A. M.; Gibbons, P. C.; Buhro, W.E. Science 1995, 270, 1791-1794.

5. (a) Trentler, T. J.; Goel, S. C.; Hickman, K. M.; Viano, A.M.; Chiang, M. Y.; Beatty, A. M.; Gibbons, P. C.; Buhro, W. E. J. Am. Chem. Soc. 1997, 119, 2172-2181. (b) Wang, F. D.; Dong, A. G.; Sun, J. W.; Tang, R.; Yu, H.; Buhro, W. E. Inorg. Chem. 2006, 45, 7511-7521. (c) Wang, F. D.; Yu, H.; Li, J. B.; Hang, Q. L.; Zemlyanov, D.; Gibbons, P. C.; Wang, L. W.; Janes, D. B.; Buhro, W. E. J. Am. Chem. Soc. 2007, 129, 14327-14335.

6. (a) Micic, O. I.; Curtis, C. J.; Jones, K. M.; Sprague, J. R.; Nozik, A. J. J. Phys. Chem. 1994, 98, 4966-4969. (b) Micic, O. I.; Sprague, J. R.; Curtis, C. J.; Jones, K. M.; Machol, J. L.; Nozik, A. J.; Giessen, H.; Fluegel, B.; Mohs, G.; Peyghambarian, N. J. Phys. Chem. 1995, 99, 7754-7759. (c) Lucey, D. W.; MacRae, D. J.; Furis, M.; Sahoo, Y.; Cartwright, A. N.; Prasad, P. N. Chem. Mater. 2005, 17, 3754-3762.

7. (a) A. Swanson et. al. Natl. Bur. Stand. (U.S.) 1955, Circ. 539, 5, 26.

(b) JCPDS 00-006-0416

8. Narayanaswamy, A.; Xu, H.; Pradhan, N.; Kim, M.; Peng, X. J. Am. Chem. Soc. 2006, 128, 10310-10319. 
9. (a) Massidda, S., Continenza, A., Freeman, A.J., de-Pascale, T.M., Meloni, F., Serra, M. Phys. Rev. B 1990, 41, 12079. (b) JCPDS 01-070-2513

10. (a) Duan, X. F.; Huang, Y.; Cui, Y.; Wang, J. F.; Lieber, C. M. Nature 2001, 409, 66-69.(b) Wang, J. F.; Gudiksen, M. S.; Duan, X. F.; Cui, Y.; Lieber, C. M. Science 2001, 293, 1455-1457. (c) Gudiksen, M. S.; Wang, J. F.; Lieber, C. M. J. Phys. Chem. B 2002, 106, 4036-4039.

11. (a) Novotny, C. J.; Yu, P. K. L. Appl. Phys. Lett. 2005, 87, 203111/1-203111/5.

(b) Fanfair, D. D.; Korgel, B. A. Crystal Growth and Design 2005, 5, 1971-1976.

12. Karanth, D.; Fu, H. Phys. Rev. B 2006, 74, 155312/1-155312/10.

13. Xu, S.; Kumar, S.; Nann, T. J. Am. Chem. Soc. 2006, 128, 1051-1054. 


\section{Chapter 5 Syntheses of Indium and Bismuth Nanoparticles}

\section{Introduction}

This chapter describes the preparation of indium and bismuth metal nanoparticles. The metal nanoparticles were prepared by reducing inorganic salts including indium trichloride and bismuth nitrate pentahydrate with hydride reducing agents. The motivation of the research was to find new synthetic routes to prepare nanoparticles of metal with low melting point $\left(<300{ }^{\circ} \mathrm{C}\right)$ such as that of indium $\left(157^{\circ} \mathrm{C}\right)$ and bismuth $\left(271{ }^{\circ} \mathrm{C}\right) .{ }^{1}$ The melting points of these nanoparticles are expected to be lower than their bulk which are already relatively low when compared to the other metals. ${ }^{2-4}$ The nanoparticles of indium and bismuth are of interest because of their uses as "seeds" that "catalyzed" the growth of semiconductors nanowires via solution liquid solid (SLS) mechanism. ${ }^{5-9}$ Detailed descriptions of SLS growth is presented in section 1.3 in chapter 1. During SLS growth, the metal seeds become molten droplets at a reaction temperature higher than their melting points. The metal droplets form alloy droplets with the semiconductor component by dissolution. Upon reaching supersaturation, the semiconductor component crystallizes out from alloy droplets and grows into nanowires. The diameters of the nanowires are controlled by and approximately matched by that of the metal seeds used. Thus, to prepare nanowires with a uniform diameter distribution, it is best to start a synthesis with metal seeds that have a uniform diameter. The lengths of the nanowires are governed by factors including the supply of precursors (from which the semiconductor forms) and the actual solubility of the semiconductor component in the metal droplets (under the reaction conditions). In general, the more precursors available results in longer nanowires.

In literature there are very limited reports on the preparations of small $(<10 \mathrm{~nm})$ monodisperse indium and bismuth nanoparticles. The best syntheses were reported by Heng and coworkers. In their study organometallic compound cyclopentadienylindium $\left(\mathrm{In}\left(\mathrm{C}_{5} \mathrm{H}_{5}\right)\right)$ and tris(bis(trimethylsilyl)amido)bismuth $\left(\mathrm{Bi}\left[\mathrm{N}\left(\mathrm{SiMe}_{3}\right)_{2}\right]_{3}\right)$ were injected into a hot solution containing surfactants and gold clusters of the formula $\mathrm{Au}_{101}\left(\mathrm{PPh}_{3}\right)_{21} \mathrm{Cl}_{5}$ which have $1.5 \mathrm{~nm}$ nanometer Au cores. ${ }^{10}$ 
The gold clusters served as seeds from which heterogeneous growth of indium and bismuth nanoparticles occurred. The thermal decomposition process creates a single nuclei formation event and subsequent growth of the nuclei on seeds with uniform size results in monodispersed nanoparticles. The actual size of a particular batch of nanoparticles could be controlled by specifying the number of seeds used, seed size and the amount of bismuth and indium precursor used.

The aim of this research was to prepare small monodispersed indium and bismuth nanoparticles from hydride reductions of inorganic salts instead of thermal decompositions of orgaonometallic compounds which may be relatively difficult to prepare. Based on thermodynamic argument in the form of electromotive force (EMF), both lithium aluminium hydride and lithium borohydride are capable of reducing indium ions $\left(\mathrm{In}^{3+}\right)$ to indium metal $\left(\mathrm{In}^{0}\right)$ as shown in scheme 5.0. Five experiments were presented with the research work on indium metal nanoparticles described in section 5.11-5.14 and that of bismuth nanoparticles in section 5.2.

Scheme 5.0 Standard electromotive forces (EMF) of two theoretical electrochemical reactions. EMF are calculated based on values from reference 1.

$$
\begin{array}{ll}
3 \mathrm{LiAlH}_{4}+\mathrm{In}^{3+} \rightarrow \mathrm{In}+3 \mathrm{Li}^{+}+3 \mathrm{AlH}_{3}+1.5 \mathrm{H}_{2} & \mathrm{E}^{\circ}+0.61 \mathrm{~V} \\
3 \mathrm{LiBH}_{4}+9 \mathrm{H}_{2} \mathrm{O}+\mathrm{In}^{3+} \rightarrow \ln +3 \mathrm{Li}^{+}+3 \mathrm{~B}(\mathrm{OH})_{3}+10.5 \mathrm{H}_{2} & \mathrm{E}^{\circ}+1.41 \mathrm{~V}
\end{array}
$$

\subsection{Indium Metal Nanoparticles}

The metal to hydride molar ratio of for indium nanoparticles and bismuth nanoparticles synthesis was $1: 3$ and 1:5 respectively.

\subsection{Reaction of Indium Trichloride and Lithium Borohydride in Toluene (Experiment 1)}

An experiment was carried out in attempt to reduce indium trichloride with lithium borohydride in toluene. Tetraoctylammonium bromide was used as a surfactant and also helped solubilise indium trichloride in toluene. After the lithium borohydride was added the reaction proceeded to give a cloudy white solution after all gaseous species have escaped. No indium metal nanoparticles were produced. A black solution would be 
expected if indium nanoparticles have formed. Refluxing the solution at $\sim 110{ }^{\circ} \mathrm{C}$ did not lead to any significant colour change apart from the clearing-up of the initial cloudiness observed at room temperature. This experiment failed to produce indium metal nanoparticles. The white precipitate was speculated to be a polymeric indium hydride of the formula $\mathrm{InH}$. Based on an early report by Wilberg et. al. polymeric indium hydride $\mathrm{InH}$ can be obtained from the room temperature decomposition of compound $\operatorname{In}\left(\mathrm{H}_{3}\right)_{\mathrm{x}}$ which is an associated form of indane. ${ }^{11}$ The polymeric hydride was found to be insoluble in ether and to thermally decompose in vacuo above $340{ }^{\circ} \mathrm{C}$ to form indium

metal and hydrogen. ${ }^{11}$ Indane $\left(\mathrm{InH}_{3}\right)$ may have been formed as an intermediate species from the of lithium borohydride and indium trichloride. The insolubility and thermal stability of the polymeric indium hydride InH would explain the behaviour observed for the white solid. However, no further investigation was launched into the chemical composition of the white solid and there was no evidence to corroborate that the white solid being polymeric indium hydride of the formula $\mathrm{InH}$. Dimeric indium hydride $\mathrm{In}_{2} \mathrm{H}_{6}$, theoretically predicted to be thermodynamically unstable has been prepared in a solid argon matrix. ${ }^{12,13}$ Thus, there is a possibility that polymeric indium hydride InH exists at room temperature.

\subsection{Reaction of Indium Trichloride and Lithium Aluminium Hydride in Toluene (Experiment 2)}

An experiment was carried out in attempt to reduce indium trichloride with lithium borohydride in toluene. Tetraoctylammonium bromide was used as a surfactant that helped solubilising indium trichloride in toluene. The reaction proceeded to a light grey to white solution after all gaseous species have escaped. A fine silvery solid precipitate was observed after the solution was allowed to settle. The precipitate was believed to be fine aluminium metal powder.

There is no direct evidence of the formation of indium metal nanoparticles as the colour of the solution was only light grey to white. A deep grey to black solution would be expected if indium nanoparticles have formed. It was speculated that indium may have been formed but only in a low yield. This experiment showed that lithium aluminium hydride reacts with indium trichloride in toluene to produce a fine silvery solid as the major product which is believed to be aluminium metal. No sufficiently strong evidence 
is available to prove the presence of indium metal nanoparticles. It was suspected that the same polymeric indium hydride $\mathrm{InH}$ has been formed instead of indium metal. Aluminium metal was believed to form from the decomposition of alane $\left(\mathrm{AlH}_{3}\right)$ to aluminium and hydrogen gas. Due to the synthesis was carried out in a closed environment, allane gas would accumulate to a concentration sufficiently high and a time sufficiently long for the decomposition process to occur.

\subsection{Reaction of Indium Trichloride and Lithium Borohydride in Isobutyamine (Experiment 3)}

An experiment was carried out treating a mixture of indium trichloride, isobutylamine and trioctylphosphine oxide with a solution of lithium borohydride in tetrahydrofuran. A clear colourless solution was obtained after all gaseous species have escaped. There were no indium metal nanoparticles formed at this point. The solution was heated to reflux at $65-70{ }^{\circ} \mathrm{C}$ and the colour of the solution changed gradually from yellow to brown and finally black. The black colour of the solution indicated the formation of indium metal nanoparticles. A black solid was isolated after purification in an inert atmosphere. 

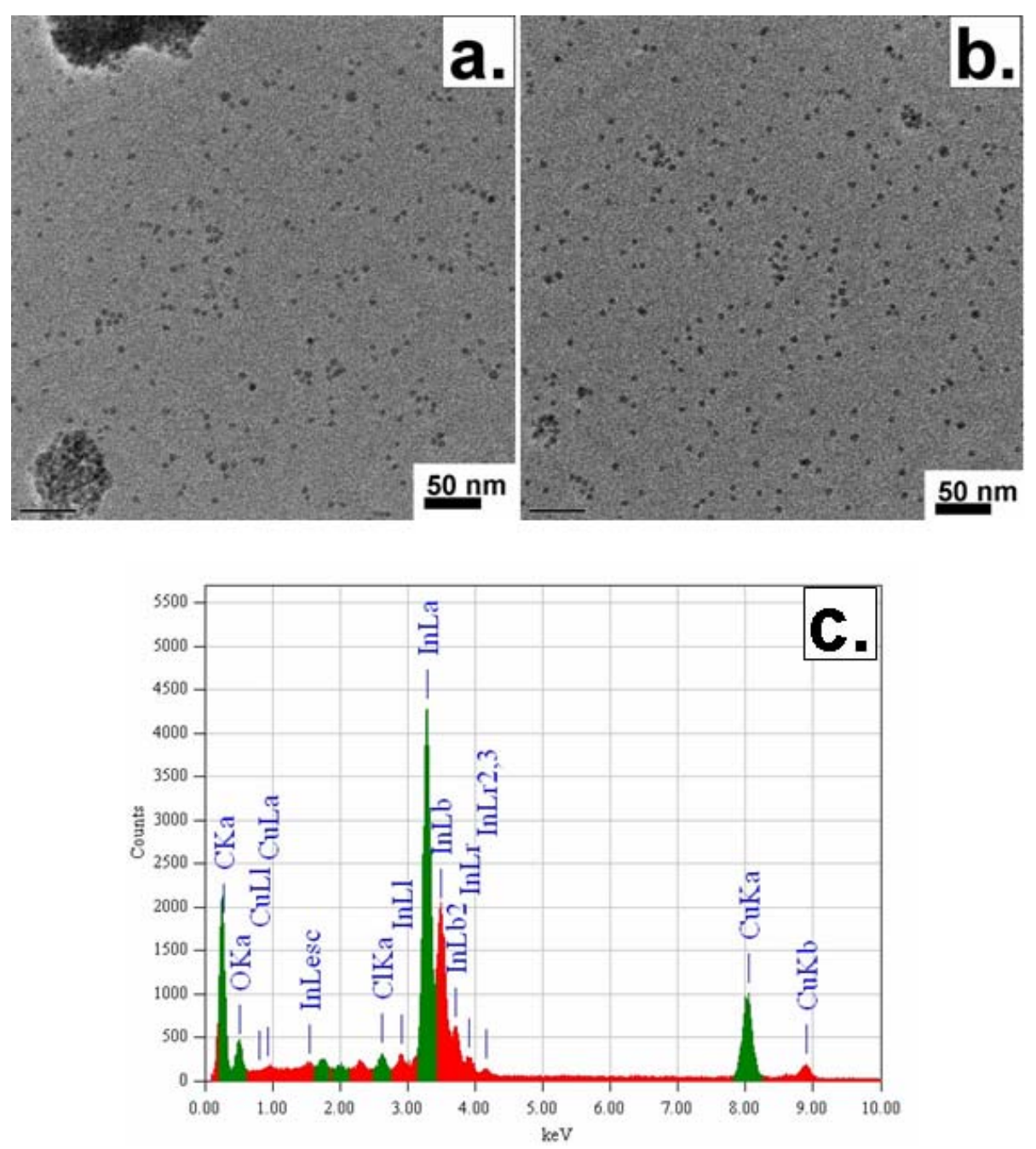

Figure 5.130 TEM images and an EDS spectrum. (a) \& (b) groups of nanoparticles with similar diameters and (c) an EDS analysis showed the particles were constituted of predominantly indium.

As may be seen in figure $5.130 \mathrm{a}$ and $\mathrm{b}$ the solid is constituted of nanoparticles of $\sim 6$ $\mathrm{nm}$. The nanoparticles were similar in size and have an average diameter of $6 \pm 1 \mathrm{~nm}$ $(\sigma=16 \%)$. The particles presented were segregated particles but there was a strong tendency of the particles to form large agglomerated structures (figure 5.130 a). EDS analysis showed the nanoparticles were composed of indium (figure $5.130 \mathrm{c}$ ). From SAED the nanoparticles were found to be amorphous. This is probably due to the melting of these nanoparticles interacting with the electron beam during TEM analysis. The particles also appeared amorphous under HRTEM imaging conditions (inset figure 5.131). 


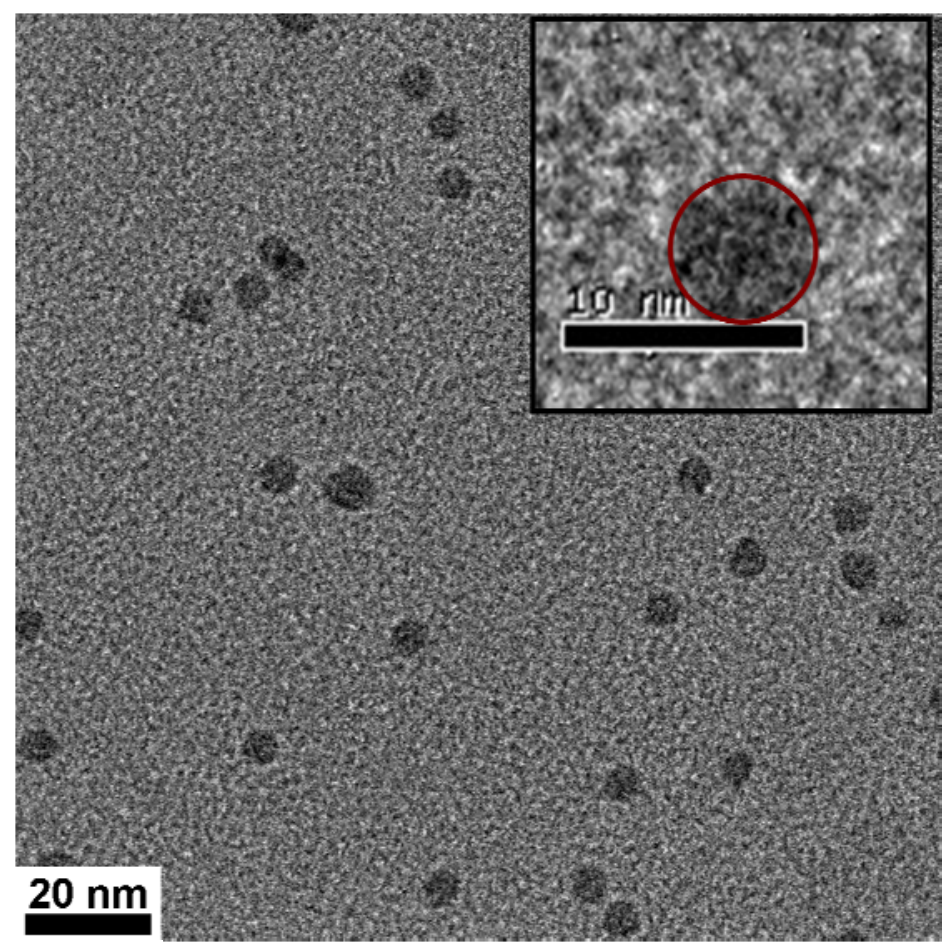

Figure 5.131 A TEM image showed segregated nanoparticles of similar size. The particles appeared amorphous as represented by the particle shown as the inset.

The nanoparticles prepared have a narrow diameter distribution as may be suggested by the standard deviation being $\sim 16 \%$ of the nanoparticle mean diameter. This is not as monodispersed as those of $7-10 \%$ deviations reported in the literature. ${ }^{10}$ However, this method is relatively simple and uses relatively common reagents. Heat is required to form indium metal nanoparticles and prolonged refluxing helps in making the particle diameter more uniform. In this experiment indium metal nanoparticles were successfully prepared from indium trichloride, lithium borohydride in isobutylamine.

The nanoparticles produced have a narrow diameter distribution and are smaller than 10 $\mathrm{nm}$ in size. The facts that gaseous product was produced upon the addition of lithium borohydride to indium trichloride in isobutylamine and that indium metal nanoparticles only formed when the solution mixture was heated to $\sim 65{ }^{\circ} \mathrm{C}$ suggested an intermediate was formed and which then when heated formed indium metal nanoparticles. The first candidate for the intermediate suggested was an isobutylamine-indane complex of with a proposed formula of $\left[\mathrm{InH}_{3}\left(\mathrm{NH}_{2} \mathrm{CH}_{2} \mathrm{CH}_{2} \mathrm{CHMe}_{2}\right)\right]$. However, the analogous trimethylamine indane complex $\left[\mathrm{InH}_{3}\left(\mathrm{NMe}_{3}\right)\right]^{12}$ is known to be stable only below 
$-30{ }^{\circ} \mathrm{C}$ therefore the intermediate is unlikely to be isobutylamine indane which is expected to be unstable at room temperature. The second candidate suggested was an isobutylamide indium complex $\left[\mathrm{In}\left(\mathrm{HNCH}_{2} \mathrm{CH}_{2} \mathrm{CHMe}_{2}\right)_{3}\right]$. Although such complex has not been reported in literature there is no sufficient evidence to reject it as a candidate.

The formation of relatively monodispersed indium nanoparticles suggested that there was a single nuclei event as a result of the thermally activation formation of indium metal. That the colour of the solution darkened only gradually over a period of one hour suggested that nascent nuclei grew slowly to form nanoparticles. The slow growth may be attributed to the interaction of surfactants (isobutylamine and trioctylphosphine oxide) and nuclei.

\subsection{Reaction of Indium Trichloride and Lithium Borohydride in N,N-diethylaniline (Experiment 4)}

An experiment was carried out treating a solution of indium trichloride and trioctylphosphine oxide in N,N-diethylaniline with a solution of lithium borohydride in tetrahydrofuran at room temperature. The solution of indium trichloride in N,Ndiethylaniline was orange similar to the colour of pure $\mathrm{N}, \mathrm{N}$-diethylaniline. Upon addition of lithium borohydride in tetrahydrofuran, a gas formed and the appearance of the solution turned from clear and orange to cloudy and yellow and finally cloudy and dark green. After being allowed to settle, a dark grey to black solid precipitate and an orange supernatant were obtained. The solid precipitate was purified with dried nitrogen-saturated solvents. A cloudy dark grey solution was obtained when the final precipitate from the purification process was suspended in toluene. A drop of the solution was dried on a TEM grid under nitrogen atmosphere. The grid was exposed to air for less than 1 minutes before the specimen was loaded to the TEM vacuum chamber. 

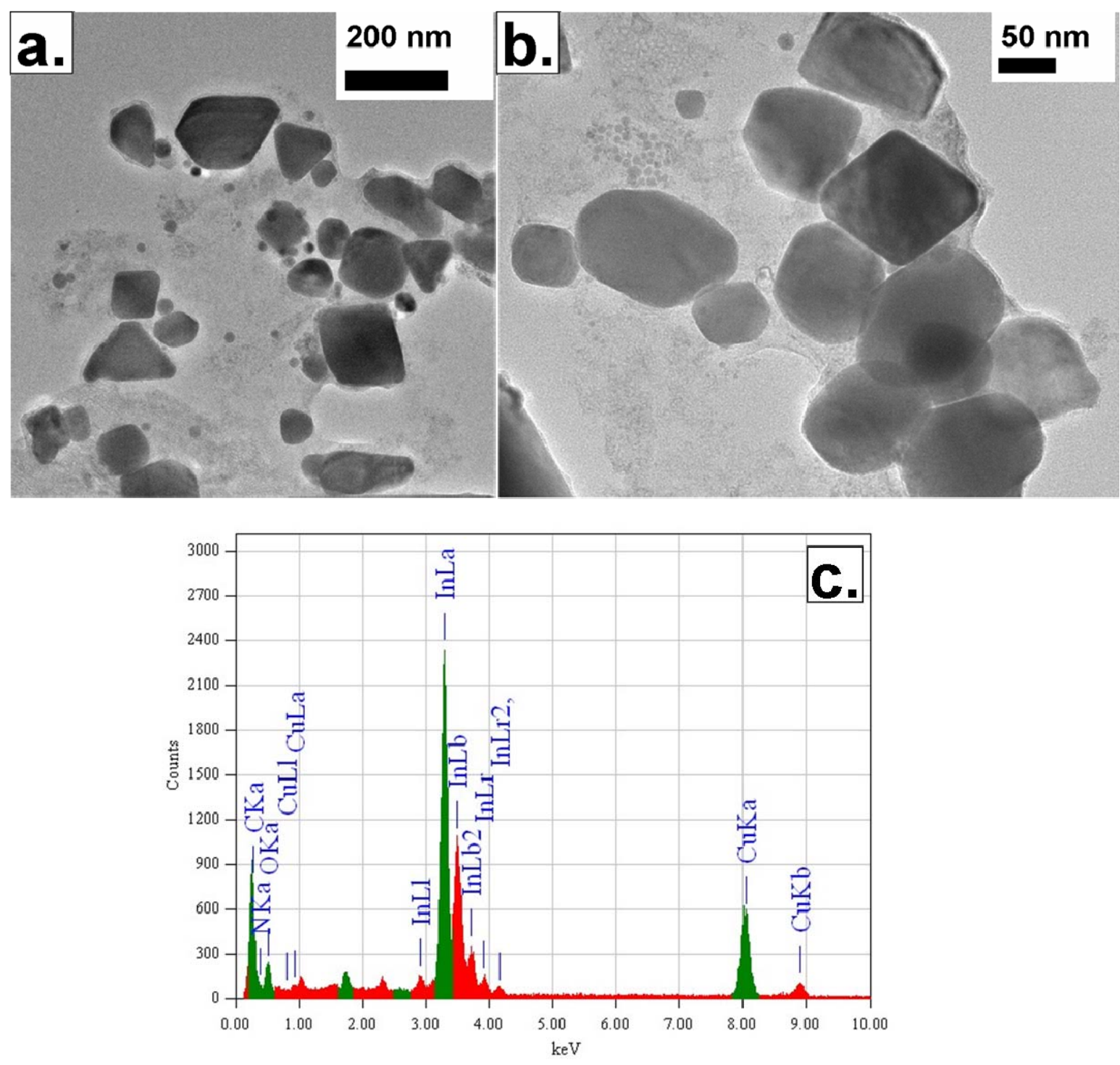

Figure 5.140 TEM images of indium nanoparticles and a corresponding EDS spectrum. 


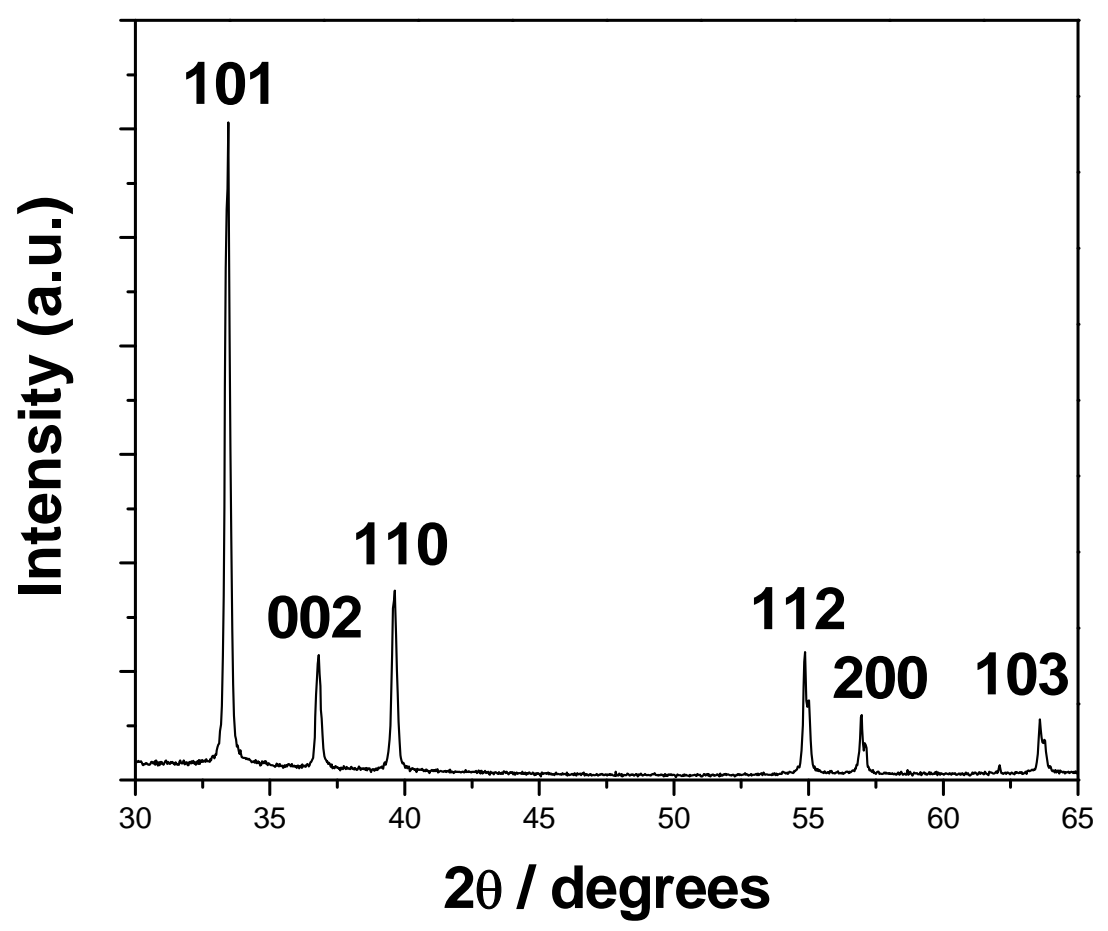

Figure 5.141 XRD pattern of indium metal nanoparticles.

As may be seen in figure 5.140 the dark grey solid precipitate was composed of nanoparticles. The size distribution of the nanoparticles was large with the smallest being $\sim 10 \mathrm{~nm}$ and the largest $\sim 100 \mathrm{~nm}$. EDS analysis showed that the nanoparticles consisted of predominantly indium. From XRD analysis (figure 5.141), the nanoparticles were found to be tetragonal indium metal. The lattice parameters $a$ and $c$ calculated for the nanoparticles using equation 5.2 derived from reference 14 was found to be $3.206 \AA$ and $4.885 \AA$ respectively. The lattice parameters were smaller than reference values of $3.2517 \AA$ and $4.9459 \AA^{15}$

$$
\frac{1}{d^{2}}=\frac{\left(h^{2}+k^{2}\right)}{a^{2}}+\frac{l^{2}}{c^{2}}----- \text { equation } 5.1
$$

This experiment proved that lithium borohydride reduces indium trichloride in N-Ndiethylaniline to form indium metal nanoparticles in the presence of trioctylphosphine oxide. The nanoparticles were polydispersed in size with the particle sizes ranged between $10 \mathrm{~nm}$ to $100 \mathrm{~nm}$. 


\subsection{Reaction of Bismuth Nitrate Pentahydrate and Lithium Borohydride in Isobutylamine (Experiment 5)}

An experiment was carried out treating a mixture of trioctylphosphine oxide and isobutylamine with a solution of lithium borohydride in tetrahydrofuran. A cloudy white solution was obtained. Hydrated bismuth nitrate was used due to it is common. The solution tuned black very quickly after a few drops of the lithium borohydride solution was added indicating a rapid reduction. The solution remained black in colour after being heated to reflux for an hour. A black solid was isolated after purification in air.
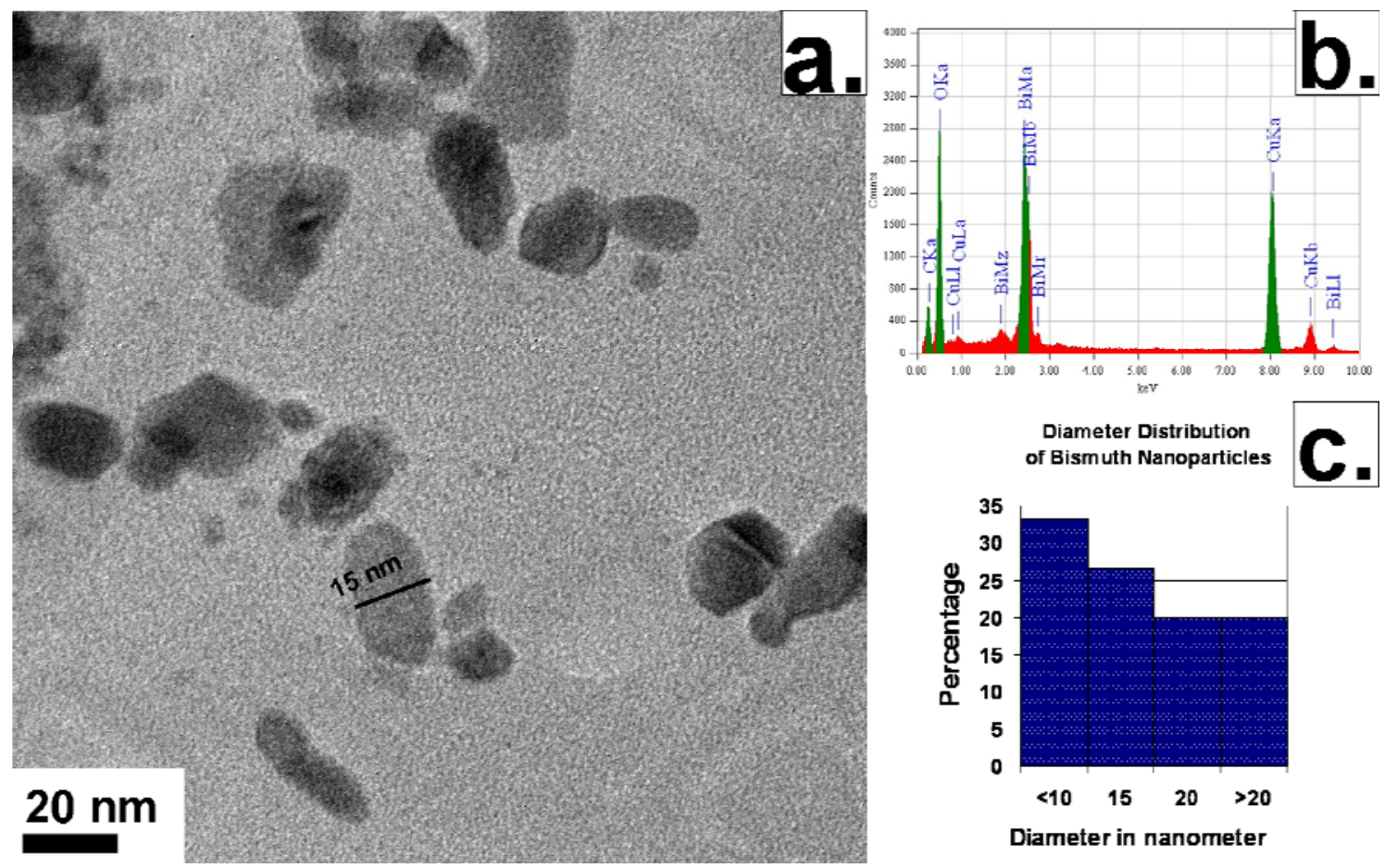

Figure 5.2 A TEM image, an EDS spectrum, and a histogram of diameter distribution. (a) A TEM image showed polydispersed nanoparticles; (b) EDS analysis showed the presence of bismuth and oxygen as dominant species and (c) broad diameter distribution of nanoparticles. 
As may be seen in figure 5.2 a the solid is constituted of nanoparticles. The nanoparticles were polydispersed in regard to their diameter distribution (figure 5.2c). Not all particles were spherical and a small percentage of them appeared as short rods. The particles diameter ranged between $7 \mathrm{~nm}$ to $32 \mathrm{~nm}$. The polydispersity of the nanoparticle diameter is believed to be due to non-homogeneous nucleation as evidenced by the formation of a black solution at the early stages of the reaction. The diameter distribution of the bismuth nanoparticles were too broad to be compared with those reported in the literature. ${ }^{10}$

From EDS (figure $5.2 \mathrm{~b}$ ), the particles contained predominantly bismuth and oxygen. The absence of phosphorous signal in the EDS spectrum proved that the oxygen signal was not due to trioctylphosphine oxide. The oxygen peak was believed to be due to surface absorption of lithium hydroxide or surface oxides formed during the purification process.

This experiment proved that bismuth nanoparticles could be prepared from bismuth nitrate pentahydrate and lithium borohydride in isobutylamine. The reduction was found to be almost immediate and a black solution was obtained before the solution was heated to reflux. The nanoparticle diameter distribution was found to be broad. From SAED, no crystalline bismuth oxide was detected although hydrated bismuth nitrate was used as the bismuth source. The water present during the reduction of bismuth nitrate was expected to have reacted with lithium borohydride to form lithium hydroxide, water and borane. 


\subsection{Discussion}

\section{Solvents Effects}

\section{Toluene versus Isobutylamine: Comparisons of Experiment 1 with Experiment 3}

In both experiments 1 and 3 indium trichoride was treated with lithium borohydride. The solution mixtures were then heated to refluxing conditions. Indium metal nanoparticles formed only in experiment 3 in which isobutylamine was used as the solvent. The differences were attributed to the different species speculated to form in both experiments. A polymeric indium hydride $(\mathrm{InH})$ was proposed to be the insoluble white solid observed in experiment 1 and for experiment 3 an isobutylamide indium complex with a proposed formula of $\left[\mathrm{In}\left(\mathrm{HNCH}_{2} \mathrm{CH}_{2} \mathrm{CHMe}_{2}\right)_{3}\right]$ was suggested to be the intermediate species. The polymeric indium hydride was reported to be thermally stable in vacuo until $340{ }^{\circ} \mathrm{C}{ }^{11}$ and such thermal stability would explain why no indium metal nanoparticles were formed in experiment 3 in which the solution had only been heated to a maximum of $\sim 110{ }^{\circ} \mathrm{C}$. The proposed complex isobutylamide indium may be thermally unstable above room temperature thus indium metal nanoparticles formed in experiment 4 in which the solution had been heated to $\sim 70{ }^{\circ} \mathrm{C}$. However, due to lack of literature evidence these two proposals remain as speculations.

\section{Isobutylamine versus N,N-diethylaniline: Comparisons of Experiment 3 with Experiment 4}

\section{Reaction Temperature}

In experiment 3 indium metal nanoparticles formed in refluxing isobutylamine whereas in experiment 4 indium metal nanoparticles formed in N-N-diethylaniline at room temperature. It was speculated in N-N-diethylaniline a reactive species was formed from lithium borohydride, N-N-diethylaniline and indium trichloride evidenced by the colour changes. The reactive species was suspected to be unstable at room temperature and therefore was expected to decompose to produce indium metal nanoparticles. The intermediate isobutylamide indium complex $\operatorname{In}\left[\mathrm{HN}\left(\mathrm{CH}_{2} \mathrm{CH}(\mathrm{Me})_{2}\right]_{3}\right.$ proposed in experiment 3 may be less reactive and only becomes thermally unstable at a temperature higher than $65^{\circ} \mathrm{C}$. 


\section{Particles Size and Shapes}

The indium metal nanoparticles formed in isobutylamine were relative small and monodispersed in size while those produced in N,N-diethylaniline were relatively large and polydispersed in size. The differences in both size and size dispersity may be explained by (i) that the amount of trioctylphosphine oxide used in N,N-diethylaniline experiment was about $\sim 40 \%$ less than the amount used in the isobutylamine experiment; (ii) that isobutylamine (being a primary amine) is a stronger surfactant than N-Ndiethylaniline toward indium metal nanoparticles and (iii) that the rate of metal particle formation was faster in N,N-diethylaniline than that in isobutylamine evidenced by the reaction speed of the development of black colour during the syntheses.

Particle size monodispersity observed in the isobutylamine system is believed to arise because the reduction reaction occurs only at $\sim 70{ }^{\circ} \mathrm{C}$ (refluxing isobutylamine) leading to a single nucleation event. Subsequent growth of these nuclei into indium metal nanoparticles, possibly in a diffusion-limited manner, ensures a sample of uniform size. In contrast, the growth of nanoparticles is appreciably faster in N,N-diethylaniline, evidenced by the much larger average size of indium nanoparticles produced. A fast but non-uniform growth of the nanoparticles may initiate an Ostwald ripening process that could result in particles size "defocusing", leading to a polydispersed sample. The faster growth of nanoparticles in N,N-diethylaniline may be attributed to a weaker solventmetal-surface interaction than is the case with isobutylamine.

\section{Different Metals}

\section{Indium Metal Seeds versus Bismuth Metal Seeds: Comparisons of Experiment 3 and Experiment 5}

\section{Particles Size}

Experiments 3 and 5 were conducted under the same conditions but with different metal precursors. Indium trichloride was used in experiment 3 and bismuth nitrate pentahydride was used in experiment 5 . Relatively uniform indium metal nanoparticles were obtained in experiment 3 whereas polydispersed bismuth nanoparticles were obtained in experiment 5 . The polydispersity of bismuth nanoparticles was due to nonhomogeneous nucleations with the processes of nucleation and growth having occurred simultaneously resulting in the formation of polydispersed nanoparticles. The reasons 
behind inhomogenous nucleations may include (i) the solution of bismuth nitrate pentahydride in isobutylamine was not a true solution but a dispersion as evidenced by the cloudy white appearance of the solution and (ii) the reduction of bismuth nitrate by lithium borohydride occurred instantly upon mixing the bismuth and borohydride species whereas in the case of indium, nanoparticles formed only when a transparent homogenous mixture was heated. 


\subsection{Conclusions}

Two new methods were developed for the synthesis of indium metal nanoparticles. The metal nanoparticles were produced from reducing inorganic metal salts with a hydride reducing agent in isobutylamine (experiment 3) and N,N-diethylaniline (experiment 4). Trioctylphosphine oxide served as a common surfactant in both experiment 3 and 4. Moderately uniform indium metal nanoparticles of $\sim 7 \mathrm{~nm}$ in size were prepared in experiment 3 and polydispersed large nanoparticles were prepared in experiment 4 . The method employed in experiment 3 was successfully adapted to the synthesis of bismuth nanoparticles (experiment 5). However, the bismuth nanoparticles formed have diameters larger than those of indium nanoparticles and were polydispersed in regard to their size distribution. 


\subsection{Experimental}

\section{Synthesis of Indium Nanoparticles (Experiment 3)}

\section{Synthesis}

In a 2-neck flask equipped with a condenser and a septum, $0.500 \mathrm{~g}$ of trioctylphosphine oxide was dissolved in $20 \mathrm{~mL}$ of isobutylamine to form a clear colourless solution. The solution containing flask was pumped under vacuum for 15 minutes to remove air. The flask was filled with nitrogen gas and the solution was allowed to warm to room temperature. The volume of the solution typically reduced to $\sim 12 \mathrm{~mL}$ in total. To the solution was added $0.166 \mathrm{~g}$ of indium trichloride, a clear colourless solution formed after being stirred for 15 minutes. To the solution was added dropwise $1 \mathrm{~mL}$ of $2 \mathrm{M}$ lithium borohydride in tetrahydrofuran in 3 minutes. Bubbles were observed in the clear colourless solution. The solution was further stirred for 12 minutes before being heated to refluxing condition in a heating mantle. The colour of the solution changed from colourless to yellow and finally dark brown/ black. After brought to reflux for $1 \mathrm{hr}$ and 6 minutes the solution was taken out from the heating mantle and allowed to cool in air resting on an aluminium heating mantle.

\section{Purification}

The particles were purified in nitrogen atmosphere. Solvents including methanol and toluene were dried across sodium sulphate anhydrous, filtered and purged with copious of nitrogen gas for one hour. Under nitrogen, $1 \mathrm{~mL}$ of the black isobutylamine solution was transferred to a centrifuged tube already containing $9 \mathrm{~mL}$ of methanol (dried over anhydrous sodium sulphate and purged with nitrogen gas). The mixture was shaken to mix and centrifuged at $4000 \mathrm{rpm}$ for 3 minutes to isolate a black precipitate. The precipitate was re-dispersed in $10 \mathrm{~mL}$ of methanol and centrifuged at $4000 \mathrm{rpm}$ to isolate a black solid. The final black precipitate was dissolved in $10 \mathrm{~mL}$ of toluene to give a dark brown to black solution. A drop of the black solution was dried on a carbon coated copper TEM grid in nitrogen atmosphere. The grid was exposed to air only during the loading of TEM grid to the TEM specimen holder. The time of exposure was measured to be $\sim 1$ minute. 


\section{Synthesis of Indium Nanoparticles (Experiment 4)}

\section{Synthesis}

In a 3-neck flask equipped with a condenser, a septum and glass temperature-probehousing, $0.3060 \mathrm{~g}$ of trioctylphosphine oxide was dissolved in $20 \mathrm{~mL}$ of $\mathrm{N}-\mathrm{N}$ diethylaniline to form a clear orange solution. The solution-containing flask was heated and pumped under vacuum for 1 hour to remove air. N,N-diethylaniline started to reflux at $65{ }^{\circ} \mathrm{C}$ under the vacuum level. The flask was filled with nitrogen gas and to the solution at $65{ }^{\circ} \mathrm{C}$ was added $0.144 \mathrm{~g}$ of indium trichloride. The resulting clear orange solution was allowed to cool to $30{ }^{\circ} \mathrm{C}$. To the solution was added dropwise $1 \mathrm{~mL}$ of

$2 \mathrm{M}$ lithium borohydride in tetrahydrofuran in 3 minutes. The colour of the orange solution changed to bright yellow and finally black upon complete addition of lithium borohydride solution.

A grey to black solid precipitate was observed to separate from the supernatant. The yellow supernatant was syringed out and discarded. To the solid was added $25 \mathrm{~mL}$ of toluene and the resulting mixture was stirred to give grey cloudy solution. The solution was centrifuged at $2500 \mathrm{rpm}$ for 5 minutes to isolate a deep grey solid. The grey solid was further washed with $25 \mathrm{~mL}$ of toluene once and with $25 \mathrm{~mL}$ of methanol twice to isolate a grey solid. A small amount of the solid was dispersed in $10 \mathrm{~mL}$ of toluene and one drop of the solution was dried on a TEM grid under an nitrogen atmosphere to prepare a specimen for TEM characterization. The grid was exposed to air only during the loading of TEM grid to the TEM specimen holder. The time of exposure was measured to be $\sim 1$ minute. For XRD measurement, the grey solid was dispersed in $\sim 2$ $\mathrm{mL}$ of toluene and the dispersion was dried over a piece of silicon wafer. 


\section{Synthesis of Bismuth Nanoparticles (Experiment 5)}

In a 2-neck flask equipped with a condenser and a septum, $0.509 \mathrm{~g}$ of trioctylphosphine oxide was dissolved in $20 \mathrm{~mL}$ of isobutylamine to form a clear colourless solution. To the solution was added $0.179 \mathrm{~g}$ of bismuth nitrate pentahydrate. A white cloudy mixture was obtained 5 minutes later. The solution-containing flask was pumped under vacuum for 15 minutes to remove air. The flask was filled with nitrogen gas and the solution was allowed to warm to room temperature. The volume of the solution typically reduced to $\sim 12 \mathrm{~mL}$. To the solution was added dropwise $1 \mathrm{~mL}$ of $2 \mathrm{M}$ lithium borohydride in tetrahydrofuran in 2 minutes. The colour of the solution turned black upon the addition of lithium borohydride. The black solution was further stirred for 12 minutes before being heated to refluxing in a heating mantle. After being brought to reflux for $1 \mathrm{hr}$ and 27 minutes the solution was taken out from the heating mantle and allowed to cool in air resting on an aluminium heating mantle.

\section{Purification}

The nanoparticles were purified in air. Solvents including methanol and toluene were dried across anhydrous sodium sulfate, filtered and purged with copious of nitrogen gas for one hour. Under nitrogen, $2 \mathrm{~mL}$ of the black solution was transferred to a centrifuged tube already containing $9 \mathrm{~mL}$ of methanol (dried over anhydrous sodium sulphate and purged with nitrogen). The mixture was shaken to mix and centrifuged at $4000 \mathrm{rpm}$ for 3 minutes to isolate a black precipitate. The precipitate was re-dispersed in $10 \mathrm{~mL}$ of methanol and the dispersion was centrifuged at $4000 \mathrm{rpm}$ to isolate a black solid. The final black precipitate was dissolved in $10 \mathrm{~mL}$ of toluene to give a dark brown to black solution. A drop of the black solution was dried on a carbon coated copper TEM grid in air for TEM characterization. 


\subsection{References}

1. (a) Aylward, G.; Findlay, T. SI Chemical Data $4^{\text {th }}$ Ed., John Wiley and Son, Australia, 1998. (b) Senoh, H; Kiyobayashi, T.; Kuriyama, N. International Journal of Hydrogen Energy 2008, 33, 3178-3181.

2. COOMBES, C. J. J. Phys. F: Metal Phys. 1972, 2, 441-449.

3. Dippel, M.; Maier, A.; Gimple, V; Wider, H.; Evenson, W. E.; Rasera, R. L.; Schatz, G. Phys. Rev. Letts. 2001, 87, 095505/1-095505/4.

4. Quaas, M.; Shyjumon, I.; Hippler, R.; Wulff, H. Z. Kristallogr. Suppl. 2007, 26, 267-272.

5. (a) Wang, F. D.; Dong, A. G.; Sun, J. W.; Tang, R.; Yu, H.; Buhro, W. E. Inorg. Chem. 2006, 45, 7511-7521.

6. Yu, H.; Buhro, W. E. Adv. Mater. 2003, 15, 416-419.

7. Nedeljkovic, J. M.; Micic, O. I.; Ahrenkiel, S. P.; Miedaner, A.; Nozik, A. J. J. Am. Chem. Soc. 2004, 126, 2632-2639.

8. Grebinski, J. W.; Hull, K. L.; Zhang, J.; Kosel, T. H.; Kuno, M. Chem. Mater. 2004, 16, 5260-5272.

9. Fanfair, D. D.; Korgel, B. A. Crys. Growth \& Des. 2005, 5, 1971-1976.

10. Yu, H.; Gibbons, P. C.; Kelton, K. F.; Buhro, W. E. J. Am. Chem. Soc. 2001, 123, 9198-9199.

11. Wiberg, E.; Dittmann, O.; Schmidt, M. Zeitschrift fuer Naturforschung 1957, 12B, $57-58$.

12. Jones, C. Chem. Comm. 2001, 2293-2298. 
13. Pullumbi, P.; Bouteiller, Y.; Manceron, L.; Mijoule, C. Chem. Phys. 1994, 185, 25-37.

14. Ladd, M.; Palmer, R. Structure Determination by X-ray Crystallography $4^{\text {th }}$ Ed., Kluwer Academic/Plenum Publishers, New York, United States of America, 2003.

15 (a) Swanson, F. Natl. Bur. Stand. (U.S) Circ 539 1954, 111, 12.

(b) JCPDS 00-005-0642.

16 (a) Sailer, G. McCarthy, North Dakota State University, Fargo, North Dakota, USA, ICDD Grant-in-Aid, 1992.

(b) JCPDS 00-044-1246. 


\section{Chapter 6 Solution Synthesis of Indium Phosphide Nanowires: Indium Trichloride and Phosphorus Pentabromide as Precursors}

\section{Introduction}

This chapter describes the development of a new method to prepare indium phosphide nanowires. The nanowires were prepared from indium trichloride, phosphorus pentabromide and lithium borohydride in solution. The aims of the research include (i) the preparation of indium phosphide in the form of nanowires with controlled lengths and diameters and (ii) the maximization of the yield of indium phosphide nanowires prepared. Techniques including PXRD, FESEM, TEM/HRTEM and PL spectroscopy were employed to characterize the products.

Indium phosphide nanowires have been previously synthesized via both vapour and solution routes. Nanowires made from vapour deposition methods such as metal-organic chemical vapour deposition (MOCVD) ${ }^{1-9}$, thermal evaporation deposition ${ }^{22,23}$ and laserassisted catalytic growth (LCG) ${ }^{10-13}$ typically were grown via vapour-liquid-solid (VLS) mechanism.

Indium phosphide nanowires were first prepared in solution by Trentler and coworkers. ${ }^{14}$ An organometallic indium compound, for example trimethylindium, was used as the indium source and tris-trimethylsilylphosphine was used as the phosphorus source. The reaction proceeded to give indium phosphide nanowires in the presence of indium nanoparticles which have a melting pointing near or lower than the reaction temperature. The growth of nanowires was governed by the metal nanoparticles which become molten liquid droplets at a reaction temperature higher than their melting points. The growth mechanism was coined solution-liquid-solid (SLS) growth and may be regarded an analogue of the VLS growth mechanism. ${ }^{14-16,20}$ 
Phosphines including tris-trimethylsilylphosphine are commonly used in the preparation of SLS-grown indium phosphide nanowires. Most phosphines are highly toxic and pyrophoric and may be costly. These issues become particularly problematic when scaling up the syntheses. The motivation of this research was to establish a nonphosphine route to prepare indium phosphide nanowires via SLS growth. Phosphorus pentabromide was chosen to be the alternate phosphorus source and the reduction of indium trichloride and phosphorus pentabromide by lithium borohydride in trioctylphosphine the alternate route to indium phosphide.

Phosphorus pentabromide is a crystalline solid at room temperature which dissolves in hexadecane to form a stable solution. Among several advantages, it serves as a less toxic and non-flammable phosphorus source more suited to scale-up synthesis. Indium trichloride was used for its availability. Lithium borohydride was chosen as the strongest reducing agent of the borohydride series. Lithium aluminium hydride was avoided as there is a possibility of aluminium contamination.

Trioctylphosphine was chosen for various reasons: (i) being a liquid at room temperature it could be transferred with ease; (ii) indium trichloride dissolves in trioctylphosphine easily to yield a stable solution; (iii) it is thermally stable; (iv) it does not react with lithium borohydride; (v) it has been widely used to prepare indium phosphide nanocrystals in the literature ${ }^{16}$; (vi) being sterically bulky it should provide sufficient steric stabilization to prevent the aggregation of nanostructures; (vii) it can be easily removed by organic solvent wash; (viii) it may be oxidized in air to form trioctylphosphine oxide (TOPO). The oxidation may be advantageous as it may help preventing the post-synthesis oxidation of indium phosphide.

A total of 40 experiments have been carried out and amongst these eleven experiments have been selected and the corresponding results are described in sections 6.1, 6.2 and 6.31 to 6.39. Brief experimental details of the eleven experiments can be found in table 6.1 following this paragraph. Experiment 1 and 2 were control experiments in which the reaction of phosphorus pentabromide and lithium borohydride and the reaction of indium trichloride and phosphorus pentabromide were studied respectively. In experiments 3-11 lithium borohydride, indium trichloride and phosphorus pentabromide were reacted under specific reaction conditions and various forms of 
indium phosphide including nanowires have been prepared. By studying these experiments, a better understanding of the reaction mechanism has been achieved and this mechanism is presented in section 6.33. The effects of various reaction parameters on the formation and morphology of indium phosphide are discussed in section 6.4. 
Table 6.1 Table of experiments.

\begin{tabular}{|c|c|c|c|c|c|c|c|}
\hline Experiment & Tempe & ture in ${ }^{\circ} \mathrm{C}$ & $\begin{array}{c}\text { Solvent/ } \\
\text { Surfactant }\end{array}$ & $\begin{array}{l}\text { Time } \\
\text { in hrs }\end{array}$ & Method & Seeds & Products \\
\hline & Injection & Growth & & & & & \\
\hline 1 & 25 & 25 & $\begin{array}{l}\mathrm{HEDE}^{* 1 /} \\
\mathrm{TOAB}^{2} \\
\end{array}$ & 1 & $\begin{array}{l}\text { 1. } \mathrm{PBr}_{5} \text { in HEDE/ TOAB solution. } \\
\text { 2. } \mathrm{LiBH}_{4} \text { solution added dropwise to (1). }\end{array}$ & N/A & $(\mathrm{PH})_{\mathrm{x}}$ \\
\hline 2 & 25 & 25 & $\begin{array}{l}\text { HEDE / } \\
\text { TOAB }\end{array}$ & 36 & $\begin{array}{l}\text { 1. Solution of } \mathrm{InCl}_{3} \text { and } \mathrm{PBr}_{5} \text { in } \mathrm{HEDE} / \mathrm{TOAB} \text { was } \\
\text { stirred. }\end{array}$ & N/A & $\begin{array}{l}\text { Insoluble } \\
\text { compound. }\end{array}$ \\
\hline 4 & 250 & $250-280$ & TOP & 10 & $\begin{array}{l}\text { 1. } \mathrm{InCl}_{3} \text { was dissolved in TOP. } \\
\text { 2. } \mathrm{PBr}_{5} \text { in } \mathrm{HEDE} / \mathrm{TOAB} \text { was treated with } \mathrm{LiBH}_{4} \text {. } \\
\text { 3. (2) was injected to }(1) \text { at } 250{ }^{\circ} \mathrm{C} \text { and allowed } \\
\text { to react for } 10 \text { hours. }\end{array}$ & No & $\begin{array}{l}\text { InP nanowires, } \\
\text { nanorods , } \\
\text { nanofibers. }\end{array}$ \\
\hline 5 & 250 & $240-280$ & TOP & 3 & $\begin{array}{l}\text { 1. } \mathrm{InCl}_{3} \text { and } \mathrm{PBr}_{5} \text { was mixed in } \mathrm{HEDE} / \mathrm{TOAB} \\
\text { solution. } \\
\text { 2. (1) was treated with } \mathrm{LiBH}_{4} \text { and then pumped } \\
\text { under vacuum. } \\
\text { 3. Two } 0.8 \mathrm{~mL} \text { of (2) was injected into TOP and } \\
\text { allowed to react for } 3 \text { hours. }\end{array}$ & No & $\begin{array}{l}\text { Aggregated InP } \\
\text { nanorods and } \\
\text { nanorods with } \\
\text { In seeds }\end{array}$ \\
\hline 6 & 205 & 198 & $\begin{array}{l}\mathrm{NNDA}^{* 4} / \\
\text { TOP }\end{array}$ & 2 & $\begin{array}{l}\text { 1. } \mathrm{InCl}_{3} \text { in } \mathrm{NNDA} \text { was mixed with In nanoparticles } \\
\text { 2. } \mathrm{PBr}_{5} \text { in } \mathrm{HEDE} / \mathrm{TOAB} \text { was treated with } \mathrm{LiBH}_{4} \text { in } \\
\mathrm{THF} \text {. } \\
\text { 3. (2) was added to (1) } 20 \text { minutes after (1) was } \\
\text { prepared and allowed to react for an overall of } 2 \\
\text { hours }\end{array}$ & In seeds & $\begin{array}{c}\text { Aggregated } \\
\text { structures } \\
\text { consisted of } \\
\text { short InP rods } \\
\text { In nanoparticles } \\
\text { and residue } \\
(\mathrm{PH})_{\mathrm{x}} \\
\end{array}$ \\
\hline
\end{tabular}


Chapter 6

\begin{tabular}{|c|c|c|c|c|c|c|c|}
\hline \multirow{2}{*}{ Experiment } & \multicolumn{2}{|c|}{ Temperature in ${ }^{\circ} \mathrm{C}$} & \multirow{2}{*}{$\begin{array}{l}\text { Solvent/ } \\
\text { Surfactant }\end{array}$} & \multirow{2}{*}{$\begin{array}{l}\text { Time } \\
\text { in hrs }\end{array}$} & \multirow[t]{2}{*}{ Method } & \multirow[t]{2}{*}{ Seeds } & \multirow[t]{2}{*}{ Products } \\
\hline & Injection & Growth & & & & & \\
\hline 7 & 170 & 170 & TOP & 2 & $\begin{array}{l}\text { 1. } \mathrm{InCl}_{3} \text { in } \mathrm{TOP}, \mathrm{PBr}_{5} \text { in } \mathrm{HEDE}, \mathrm{LiBH}_{4} \text { in } \mathrm{TOP} \text { and } \\
\text { In nanoparticles were mixed together. } \\
\text { 2. (1) was heated in an oil bath preheated to } 200{ }^{\circ} \mathrm{C}\end{array}$ & $\begin{array}{c}\text { In } \\
\text { seeds }\end{array}$ & $\begin{array}{l}\text { Amorphous aggregated } \\
\qquad(\mathrm{PH})_{\mathrm{x}}\end{array}$ \\
\hline 8 & 250 & 250 & TOP & 3 & $\begin{array}{l}\text { 1. } \mathrm{LiBH}_{4} \text { in TOP was mixed with In nanoparticles } \\
\text { 2. InCl } \text { in TOP mixed with } \mathrm{PBr}_{5} \text { in HEDE/TOAB. } \\
\text { 3. (2) was added to (1) slowly via a dropping funnel and } \\
\text { The mixture was allowed to react for an overall of } 3 \\
\text { hours. }\end{array}$ & $\begin{array}{c}\text { In } \\
\text { seeds }\end{array}$ & $\begin{array}{l}\text { Aggregated and } \\
\text { segregated InP } \\
\text { nanoparticles } \\
\text { nanorods } \\
\text { nanofibers and } \\
\text { nanowires } \\
\end{array}$ \\
\hline 9 & 250 & 250 & TOP & 3 & $\begin{array}{l}\text { 1. InCl } \mathrm{In}_{3} \text { in TOP was treated with a small fraction of } \\
\mathrm{LiBH}_{4} \text { in TOP. } \\
\text { 2. To (1) was added dropwise alternatively } \mathrm{PBr}_{5} \text { in } \\
\mathrm{HEDE} / \mathrm{TOAB} \text { and } \mathrm{LiBH}_{4} \text { in TOP every } 10 \text { sec for } \\
45 \text { minutes. } \\
\text { 3. (2) was allowed to react for an overall of } 3 \text { hrs. }\end{array}$ & No & $\begin{array}{l}\text { InP nanowires } \\
\text { Aggregated InP } \\
\text { nanoparticles. } \\
\text { Residue }(\mathrm{PH})_{\mathrm{x}}\end{array}$ \\
\hline 10 & 250 & $250-275$ & TOP & 3 & $\begin{array}{l}\text { 1. } \mathrm{InCl}_{3} \text { in } \mathrm{TOP} \text { was mixed with In nanoparticles. } \\
\text { 2. To (1) was added dropwise alternatively } \mathrm{PBr}_{5} \text { in } \\
\mathrm{HEDE} / \mathrm{TOAB} \text { and } \mathrm{LiBH}_{4} \text { in TOP every } 10 \text { sec for } 38 \\
\text { minutes. } \\
\text { 3. (2) was allowed to react for an overall of } 3 \text { hours. }\end{array}$ & $\begin{array}{l}\text { In } \\
\text { seeds }\end{array}$ & InP nanowires. \\
\hline 11 & 254 & 260 & TOP & 3 & $\begin{array}{l}\text { 1. } \mathrm{InCl}_{3} \text { was dissolved in TOP. } \\
\text { 2. } \mathrm{PBr}_{5} \text { dissolved in a mixture of NNDA and TOP } \\
\text { was treated with } \mathrm{LiBH}_{4} \text { to form }(\mathrm{PH})_{\mathrm{x}} \text {. } \\
\text { 3. (2) was mixed with } \mathrm{Bi} \text { nanoparticles seeds mixture. } \\
\text { 4. Multiple injections of (3) into TOP at } 254^{\circ} \mathrm{C} \text {. }\end{array}$ & $\begin{array}{c}\mathrm{Bi} \\
\text { seeds }\end{array}$ & $\begin{array}{l}\text { InP nanowires and } \\
\text { Bi residues }\end{array}$ \\
\hline
\end{tabular}

\footnotetext{
${ }^{1}$ Hexadecane; ${ }^{* 2}$ Tetraoctylammonium bromide; ${ }^{* 3}$ Trioctylphosphine; ${ }^{* 4}$ N,N-diethylaniline.
} 


\subsection{Reaction of Lithium Borohydride and}

\section{Phosphorus Pentabromide : The Formation of

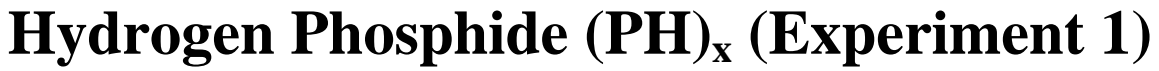

An experiment was carried out reacting phosphorus pentabromide with lithium borohydride at room temperature. A bright yellow solid was isolated after purification.

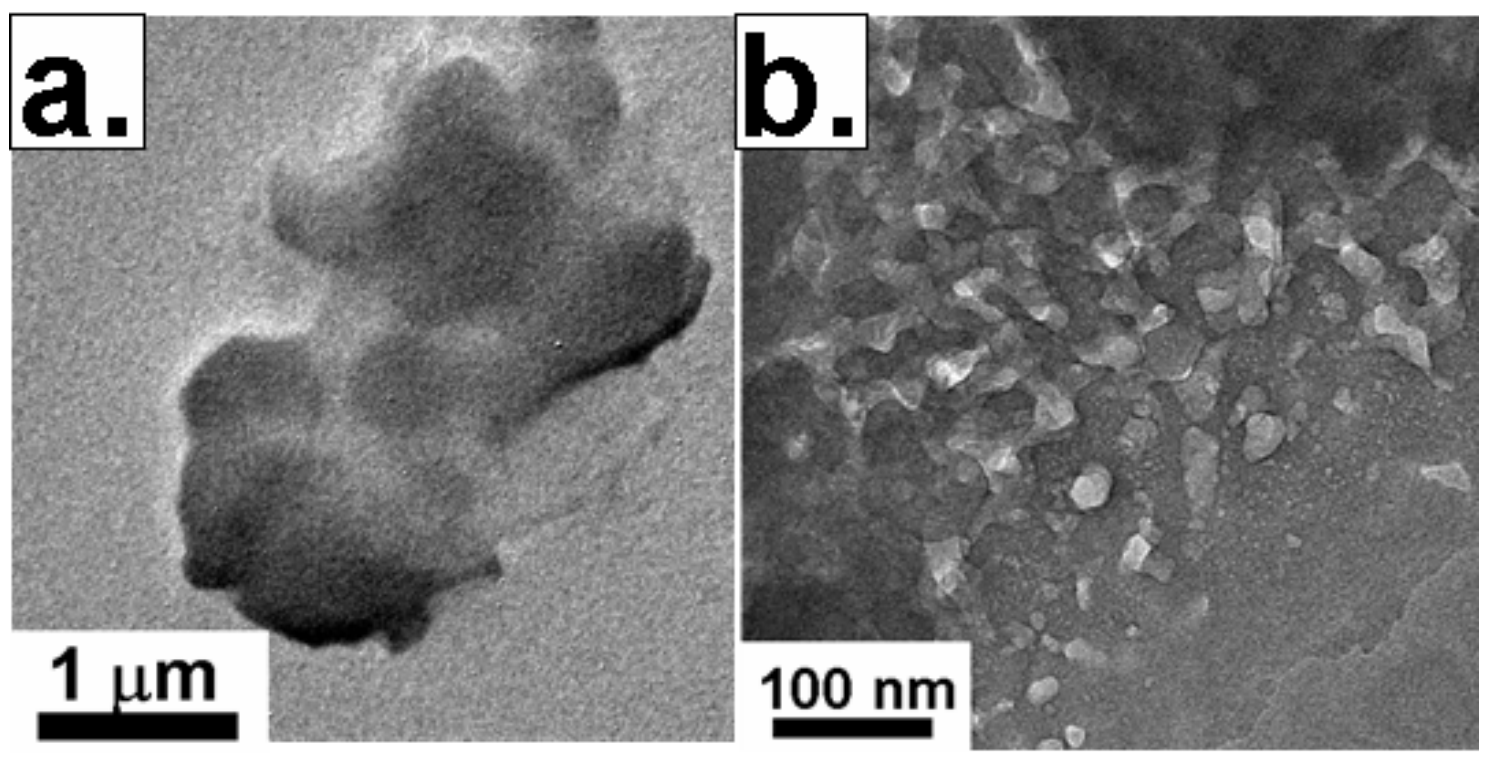

Figure 6.1 TEM images. (a) a low resolution TEM image showed a particle of micrometer dimensions and (b) a porous random network of the material that constituted the particle.

The yellow solid was studied by HRTEM. The solid was composed of nanoparticles of random shapes. The representative particle shown in figure 6.1a has a length of $\sim 2 \mu \mathrm{m}$ and diameter $\sim 1 \mu \mathrm{m}$. A closer look at the particle revealed that the thickness of the particle was not uniform. The material that constituted the particle has a porous random structure (figure $6.1 \mathrm{~b}$ ). From SAED, the particle was found to be amorphous. An EDS analysis showed that the particle consisted of mainly phosphorus.

The yellow solid is likely to be hydrogen phosphide with a formula of $(\mathrm{PH})_{\mathrm{x}}$. The assumption is based on a literature by report Wilberg and Mulle-Schiedmayer ${ }^{17}$ in the 1960s. In the report, hydrogen phosphide was prepared as a yellow to orange solid from a reaction of tribromophosphine $\left(\mathrm{PBr}_{3}\right)$ and lithium hydride $(\mathrm{LiH})$ in diethylether at 
$0{ }^{\circ} \mathrm{C}$. Scheme 6.10 represents the overall reaction to produce hydrogen phosphide from tribromophosphine and lithium hydride. Scheme 6.11 shows two consecutive reactions proposed for the production of hydrogen phosphide from phosphorus pentabromide and lithium borohydride. Tribromophosphine is first released from the reaction of phosphorus pentabromide with lithium borohydride in a 1:2 mole ratio and it further reacts with lithium borohydride in a 1:3 mole ratio to form hydrogen phosphide. By comparing the two reaction schemes the similarity between the reaction shown in scheme 6.10 and reaction (ii) shown in scheme 6.11 becomes clear. The second reaction presented in scheme 6.11 is homologous to that presented in scheme 6.10.

Scheme 6.10

$$
\mathbf{X P B r}_{3}+\mathbf{3 X L i H} \underset{{ }_{0}{ }^{\circ} \mathrm{C}}{\longrightarrow}(\mathbf{P H})_{\mathbf{X}}+\mathbf{3 X L i B r}+\mathbf{X H}_{2}
$$

\section{Scheme 6.11}

$$
\begin{aligned}
& \mathrm{CH}_{3}\left(\mathrm{CH}_{2}\right)_{14} \mathrm{CH}_{3} \\
& \text { (i) } \mathbf{P B r}_{5}+2 \mathrm{LiBH}_{4} \underset{20{ }^{\circ} \mathrm{C}}{\longrightarrow} \mathbf{P B r}_{3}+2 \mathrm{LiBr}+\mathbf{B}_{2} \mathbf{H}_{6}+\mathbf{H}_{2} \\
& \text { (ii) } \mathrm{XPBr}_{3}+3 \mathrm{XLiBH}_{4} \underset{20^{\circ} \mathrm{C}}{\stackrel{\mathrm{CH}_{3}\left(\mathrm{CH}_{2}\right)_{14} \mathrm{CH}_{3}}{\longrightarrow}}(\mathbf{P H})_{\mathrm{X}}+3 \mathrm{XLiBr}+3 \mathrm{X} / 2 \mathbf{B}_{2} \mathbf{H}_{6}+\mathbf{X H}_{2}
\end{aligned}
$$

The yellow solid consisting of phosphorus is therefore likely to be hydrogen phosphide of the formula $(\mathrm{PH})_{\mathrm{x}}$. Such yellow solid prepared from the reaction of phosphorus pentabromide and lithium borohydride will be regarded as hydrogen phosphide $(\mathrm{PH})_{\mathrm{x}}$ from this section onward in order to differentiate experiments in which phosphorus pentabromide was used directly from experiments in which the yellow solid was prepared from phosphorus pentabromide prior to reactions. 


\subsection{The Reaction of Indium Trichloride and Phosphorus Pentabromide (Experiment 2)}

An experiment was carried out mixing indium trichloride and phosphorus pentabromide in a hexadecane/toluene mixture in the presence of tetraoctylammonium bromide. Tetraoctylammonium bromide helped solubilise indium trichloride in the non-polar solvent mixture. A pale green solid precipitate was obtained after a day. The solid was re-distributed in the solvent mixture by mean of sonication. A small amount of the suspension was injected into trioctylphosphine preheated to $250{ }^{\circ} \mathrm{C}$. Immediately after the injection, a bright orange solution formed. The colour gradually faded into pale yellow. The light green solid is believed to be a coordination compound formed from indium trichloride and tribromophosphine. A search in the literature on the coordination compound however yielded no result.

Scheme 6.21 An equilibrium between $\mathrm{PBr}_{5}$ and $\mathrm{PBr}_{3}$.

$$
\left[\mathrm{PBr}_{4}\right]^{+} \mathrm{Br}^{-}{ }_{(\mathrm{s})}^{\stackrel{273 \mathrm{~K}}{\rightleftharpoons}} \mathbf{P B r}_{3(\mathrm{~g})}+\mathrm{Br}_{2(\mathrm{~g})}
$$

Phosphorus pentabromide exists in equilibrium with tribromophosphine and bromine $\left(\mathrm{Br}_{2}\right)$ as shown in scheme 6.21. ${ }^{18}$ The tribromophosphine formed is expected to coordinate to indium trichloride. Assuming an insolubility of the green solid coordination compound, the equilibrium would be driven toward the right until all phosphorus pentabromide was consumed.

After being injected into trioctylphosphine at $250{ }^{\circ} \mathrm{C}$, the coordination compound was assumed to dissociate into indium trichloride and tribromophosphine. Tribromophosphine, which has a boiling point lower than $250{ }^{\circ} \mathrm{C}$ would have evaporated leaving indium trichloride to be complexed by the surrounding trioctylphosphine. The product remaining in the pale yellow solution is likely to be a solution of indium trichloride in trioctylphosphine. No indium phosphide was observed as a product.

This experiment proved that a light green solid could be formed from indium trichloride and phosphorous pentabromide. Heating the green solid in trioctylphosphine at $250{ }^{\circ} \mathrm{C}$ did not lead to the formation of indium phosphide. 


\subsection{Reactions of Indium Trichloride, Lithium Borohydride and Phosphorus Pentabromide}

All experiments (experiments 3-11) that follow this paragraph involved the reactions of indium trichloride, phosphorus pentabromide and lithium borohydride. Unless otherwise mentioned the word "lithium borohydride" refers to a suspension of lithium borohydride in trioctylphosphine. The morphologies, crystal structures and elemental compositions of products isolated from experiments 3-11 were characterized by TEM, SAED and EDS. TEM images are presented in all result sections because product morphology is of the primary concern. The presence or absence of crystalline indium phosphide species has always been confirmed by SAED analysis although these results are not necessarily presented. Due to the vast amount of data, only in section 6.37 and 6.38 (experiments 9 and 10 respectively) are the SAED and EDS data presented

Scheme 6.30 Proposed reactions towards the formation of indium phosphide.

$$
\begin{array}{ll}
\text { (i) } & \mathrm{InCl}_{3}+3 \mathrm{LiBH}_{4} \longrightarrow \\
\text { (ii) } & \mathrm{XPBr}_{5}+5 \mathrm{XLiBH} \longrightarrow \\
\text { (iii) } & \mathrm{XIn}+(\mathrm{PH})_{\mathrm{X}} \longrightarrow(\mathrm{PH}) \mathrm{X}+5 \mathrm{XLiBr}+5 \mathrm{X} / 2 \mathrm{~B}_{2} \mathrm{H}_{6}+2 \mathrm{XH}_{2}
\end{array}
$$

Reactions leading to the formation of indium phosphide are proposed and presented in scheme 6.30. Indium metal and hydrogen phosphide are first produced from the reductions of indium trichloride and phosphorus pentabromide by lithium borohydride. The reduction of hydrogen phosphide by indium metal leads to the formation of indium phosphide. 


\subsection{Hot Injection of A Pre-reacted Mixture of Indium}

\section{Trichloride, Phosphorus Pentabromide and Lithium}

\section{Borohydride (Experiment 3)}

An experiment was carried out treating a mixture of indium trichloride and phosphorus pentabromide in hexadecane with a solution of lithium borohydride in tetrahydrofuran. A green viscous liquid was obtained. The liquid was injected into trioctylphosphine at $250{ }^{\circ} \mathrm{C}$ to form a brown solution. A brown solid was isolated after purification.

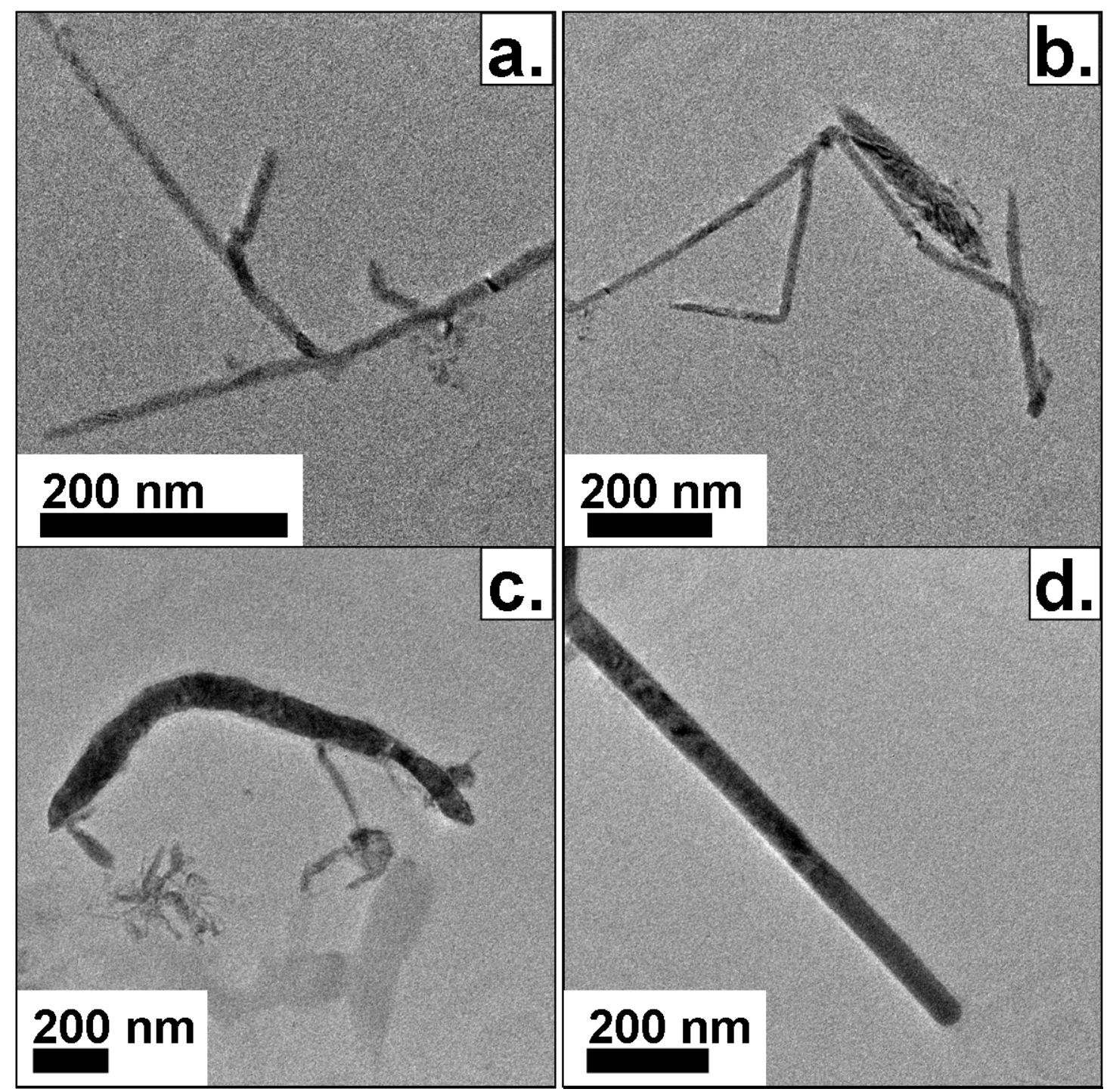

Figure 6.31 TEM images of various species of nanowires observed. (a) branched nanowires; (b) nanowires with bends and a cigar-shape nanowire; (c) a large nanowire with twists and (d) a straight nanowire. 
The identity of the gel was unclear but it should contain hydrogen phosphide based on the knowledge learned from experiment 1. It was unclear whether indium trichloride was reduced by lithium borohydride in the pre-reacted mixture at room temperature. A black solution would be expected had indium metal nanoparticles already formed in the mixture.

As may be seen in figure 6.31, the solid is constituted of nanowires. From SAED, the nanowires were found to be cubic face centred cubic indium phosphide. Apart from straight nanowires, nanowires with branches and with twists were also observed. The branched nanowires have an average diameter of $\sim 10 \mathrm{~nm}$. The other two types of nanowires have diameters that are larger than $10 \mathrm{~nm}$. That three different species were formed in a one pot synthesis is believed to be due to the fluctuations of local reaction temperature and local concentrations of reactants.

This experiment proved that indium phosphide nanowires could be prepared from a prereacted mixture of indium trichloride, phosphorus pentabromide and lithium borohydride. No quantitative measurement on the yield of indium phosphide was calculated due to the lack of knowledge of the identity and concentration of the prereacted mixture. It was therefore proposed that the reaction should be carried out by adding the reagents separately when subjected to reaction. The knowledge of the concentrations of the reagents would be beneficial in the optimization of reaction conditions. 


\subsection{Reactions of Indium Trichloride, Hydrogen Phosphide and Lithium Borohydride (Experiment 4)}

An experiment was carried out treating a solution of indium trichloride in trioctylphosphine with a mixture of hydrogen phosphide and lithium borohydride. A black solid was isolated after purification. As may be seen in figure 6.32 a, the solid consisted of a mixture of nanorods, nanowires and nanofibers. From SAED the nanofibers, nanorods and nanowires were all found to be face centred cubic indium phosphide.

To separate the nanofibers from the nanorods and nanowires, the mixture was sonicated in chloroform overnight and allowed to settle. The isolated precipitate consisted of mainly nanofibres (figure 6.32 b and c) and the supernatant of nanorods (figure $6.32 \mathrm{~d}$ f). No lattice fringes could be resolved for the nanofibres due to their thickness. The nanorods were relatively thin when compared to the nanofibres and lattices fringes could be observed as shown in figure $6.32 \mathrm{f}$. No nanowires were observed after the sonication-precipitation process. Prolonged sonication might have resulted in the breaking of long thin nanowires into shorter nanorods.

This experiment proved that crystalline indium phosphide could be prepared in forms of nanofibres and nanorods from indium trichloride and a mixture of hydrogen phosphide and lithium borohydride. Nanofibres and nanorods could be separated by sonicationprecipitation approach based on the mass difference of the two species. Particles of hydrogen phosphide in the micrometer range observed in experiment 1 gave light to the formation of indium phosphide nanofibres. It was proposed that nanofibres were formed from the reactions of large particles of hydrogen phosphide with indium trichloride and lithium borohydride ie reactions (i) and (iii) in scheme 6.30 . 


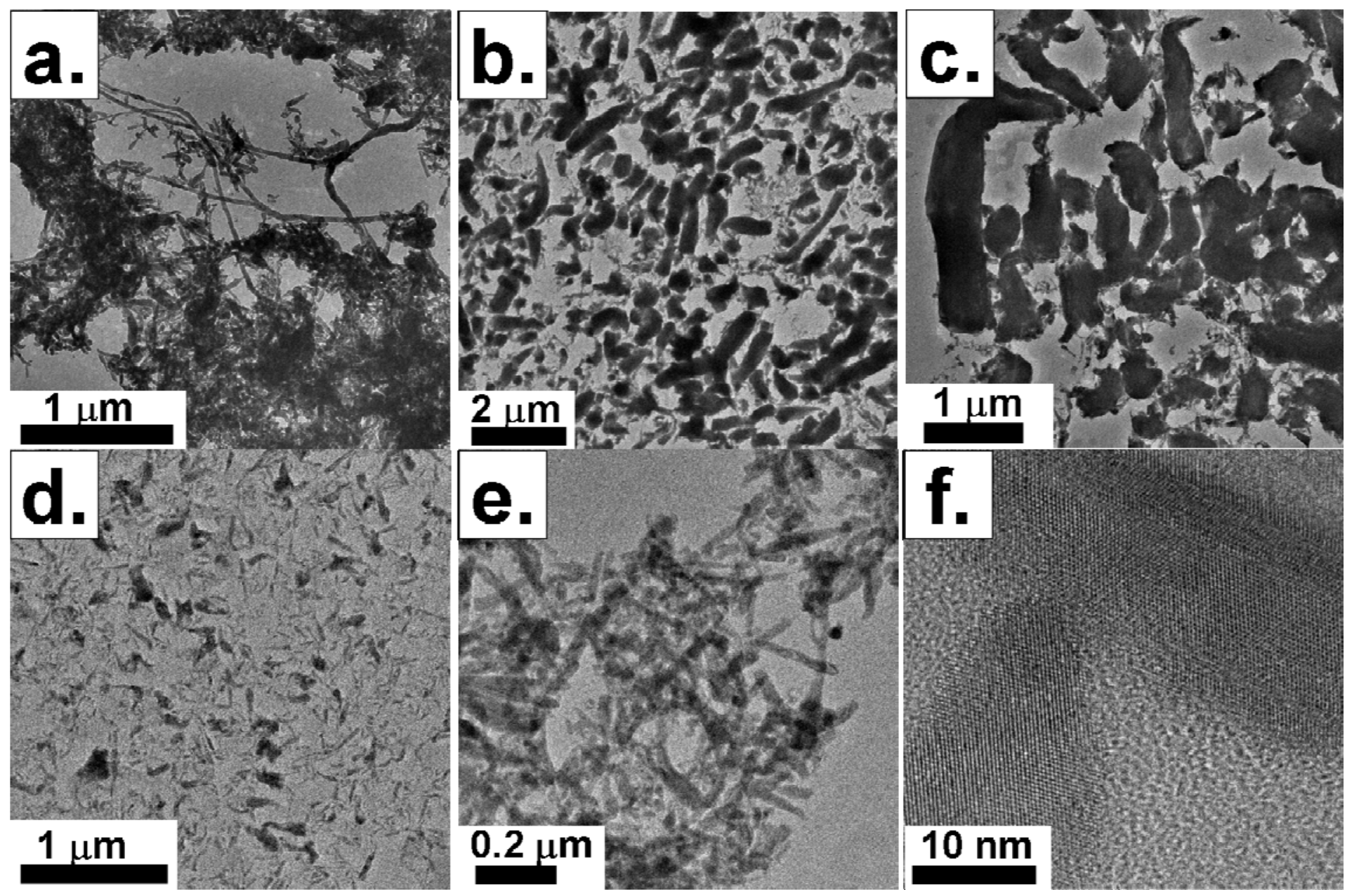

Figure 6.32 TEM images of indium phosphide nanofibers and nanorods and nanowires. (a) a mixture of nanofibers, nanorods and nanowires as synthesized; (b) nanofibers isolated after sonication-precipitation; (c) a group of nanofibers (d) nanorods isolated; (e) a group of nanorods and (f) a HRTEM image of two nanorods. 


\subsection{Injection of A Mixture of Indium Trichloride and \\ Phosphorus Pentabromide and Lithium Borohydride To Trioctylphosphine (Experiment 5)}

An experiment was carried out treating a mixture of indium trichloride and phosphorus pentabromide in hexadecane with a solution of lithium borohydride in tetrahydrofuran. A greyish green liquid was obtained after the removal of tetrahydrofuran in vacuo. It was believed that a part of the mixture has reacted under vacuum to form indium metal nanoparticles which gave rise to the grey colour observed. Two equal portions of the greyish green liquid were injected successively into a liquid of trioctylphosphine at $250{ }^{\circ} \mathrm{C}$ to form a brown solution. A brown to black solid was isolated after purification.

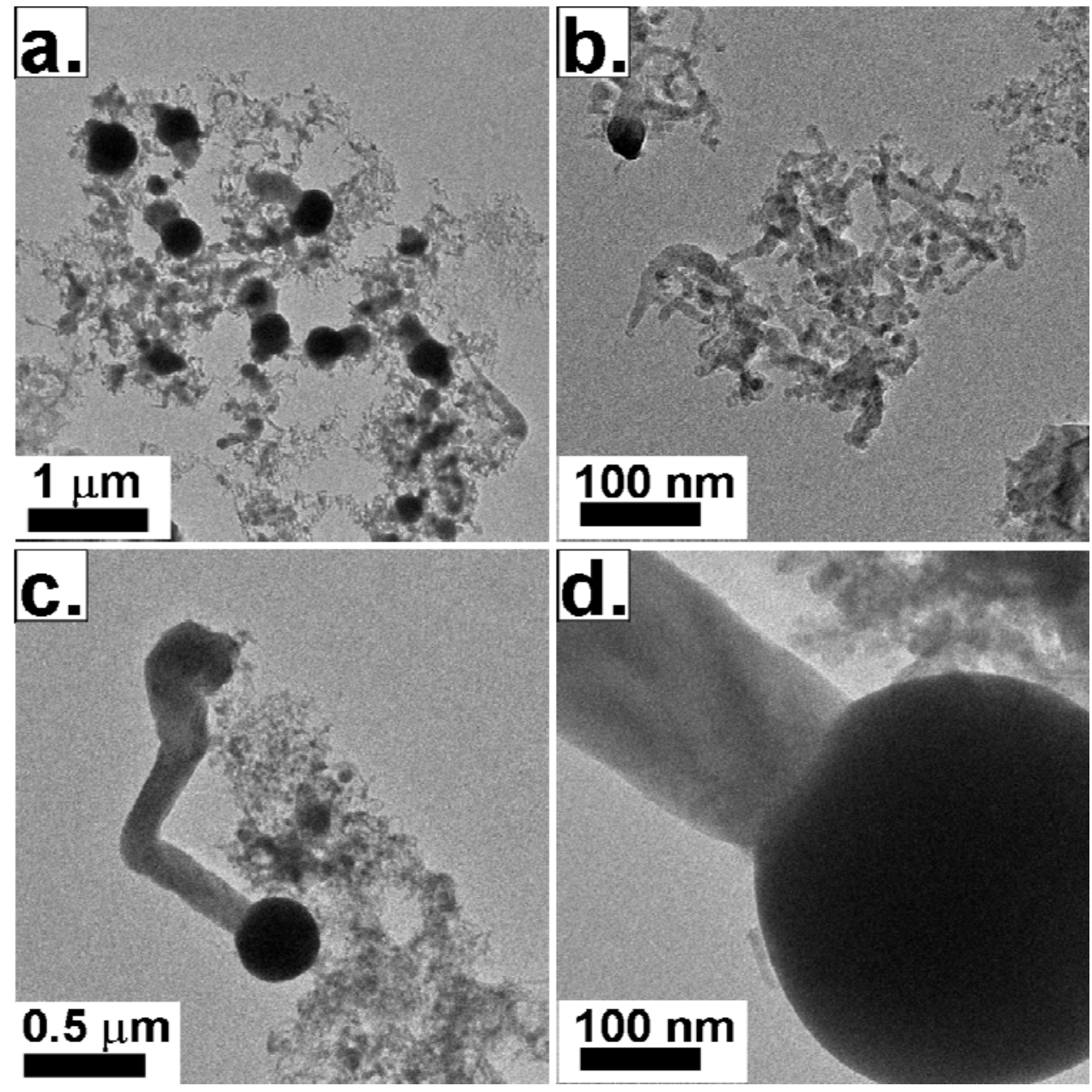

Figure 6.33 TEM images. (a) a low resolution TEM image showing short nanorods of indium phosphide with indium particles attached to their tips; (b) a group of nanorods; (c) a bent nanorod with with a metal and (d) a close look at the indium particle /indium phosphide nanorod intersection. The particle and the rod are of one singular unit. 
The solid was studied under TEM. As may be seen in figure 6.33 the solid consisted of nanorods of indium phosphide with indium particles attached to their tips, aggregated thin nanorods and some nanoparticles. From EDS the spherical tips of the mushroomlike structures were found to be constituted of dominantly indium. From SAED the tips were found to be amorphous. The rods growing out of the tips were confirmed by SAED analysis to be comprised of face centre cubic indium phosphide. The presence of mushroom-like structures indicated the nanowires were grown via SLS growth.

A detailed description of SLS growth is presented in section 1.3 in chapter 1 . In brief, SLS growth occurs when indium and phosphorus monomers dissolve into the molten metal seeds and, upon reaching supersaturation, crystallize out as indium phosphide in a specific growth direction leading to the formation of an indium phosphide nanowire. ${ }^{14,15 a, 15 b, 19}$ In this system, it is not possible for us to tell whether indium and phosphorus exist as separate monomers that combine in an indium metal seed or whether indium and phosphorus combine in trioctylphosphine to become a single indium phosphide monomer which then gets dissolved into the indium metal seed.

The initial greyish appearance of the gel suggests the presence of large indium metal nanoparticles. The large metal nanoparticles reacted to form the nanorods with indium particles attached to their tips. Thin nanorods of indium phosphide were also observed but without spherical particles attached to their tips. The absence of indium metal particles indicated that they have reacted to form indium phosphide. In the absence of the seeds, SLS growth stopped and led to short nanorods.

This experiment proved that indium phosphide nanorods could be prepared from a prereacted mixture of indium trichloride, lithium borohydride and phosphorus pentabromide. The nanorods with indium particles attached to their tips indicated that the nanorods were grown via SLS growth. 


\subsection{Indium Trichloride and Indium Metal Seeds in N,N- diethylaniline Treated with Hydrogen Phosphide and Lithium Borohydride (Experiment 6)}

An experiment was carried out treating a mixture of pre-synthesized indium metal seeds and indium trichloride in N,N-diethylaniline with a mixture of hydrogen phosphide in N,N-diethylaniline and lithium borohydride in trioctylphosphine. N,N-diethylaniline was chosen for (i) it is capable of dissolving indium trichloride and hydrogen phosphide and (ii) it has a boiling point of $\sim 210{ }^{\circ} \mathrm{C}$ which was considered sufficiently high to facilitate crystal formation. A brown solid was isolated after purification. The indium metal nanoparticles were of uniform diameter of $\sim 6 \mathrm{~nm}$ and were prepared as described in experiment 3 in chapter 5.
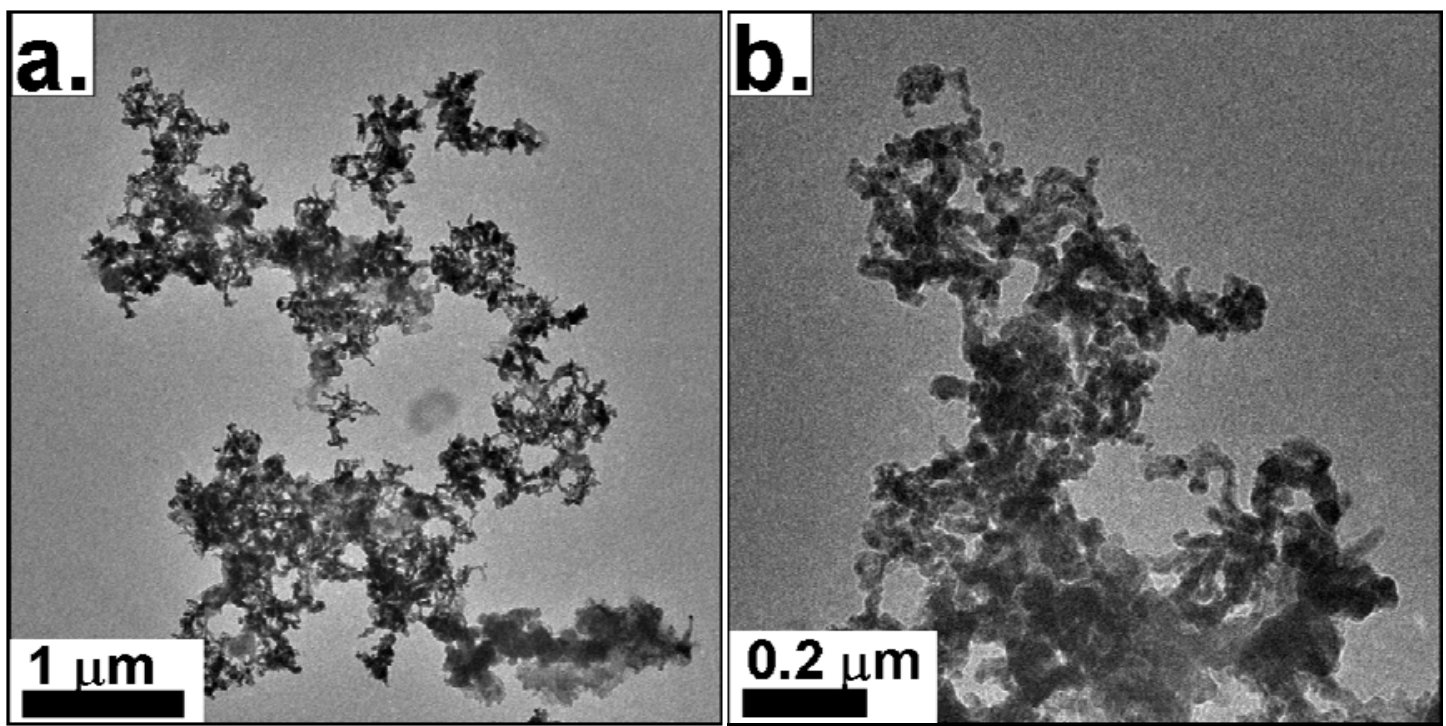

Figure 6.34 TEM images. (a) a group of aggregated nanorods and (b) an aggregate of nanorods with a high degree of bend.

The solid was studied by TEM. As may be seen in figure $6.34 \mathrm{a}$ and $\mathrm{b}$, the nanostructures are constituted of aggregates of indium phosphide nanorods with twists, nanoparticles and solid particles with no distinct shape. From SAED face centred cubic indium phosphide was found to be the only crystalline species present. It was difficult to tell whether all nanostructures were constituted of indium phosphide because of the aggregation. The indium metal seeds were expected to help to prepare indium phosphide nanowires with uniform diameters. However, no long straight indium phosphide nanowires were observed. 
This experiment proved that crystalline indium phosphide could be prepared in N,Ndiethyaniline at $\sim 200{ }^{\circ} \mathrm{C}$ from indium trichloride and a mixture of hydrogen phosphide and lithium borohydride.

\subsection{A Mixture of Indium Trichloride, Indium Metal Seeds, Phosphorus Pentabromide and Lithium Borohydride in Trioctylphosphine Heated in A Hot Oil Bath}

\section{(Experiment 7)}

An experiment was carried out as following (i) a mixture consisting of indium trichloride, indium metal seeds, phosphorus pentabromide and lithium borohydride in trioctylphosphine was prepared and (ii) the mixture was heated in an oil bath pre-heated to $200{ }^{\circ} \mathrm{C}$. A light brown solid was isolated after purification. The indium metal nanoparticles used have diameters $\sim 6 \mathrm{~nm}$ and was prepared as described in experiment 3 in chapter 5. It was hoped that by immediately heating all reagents together in a hot oil bath, uniform nanowires of indium phosphide could be prepared. In literature, the approach of heating precursors together in a hot oil bath has resulted in the preparation of indium phosphide nanowires with uniform nanowire diameters. ${ }^{19}$

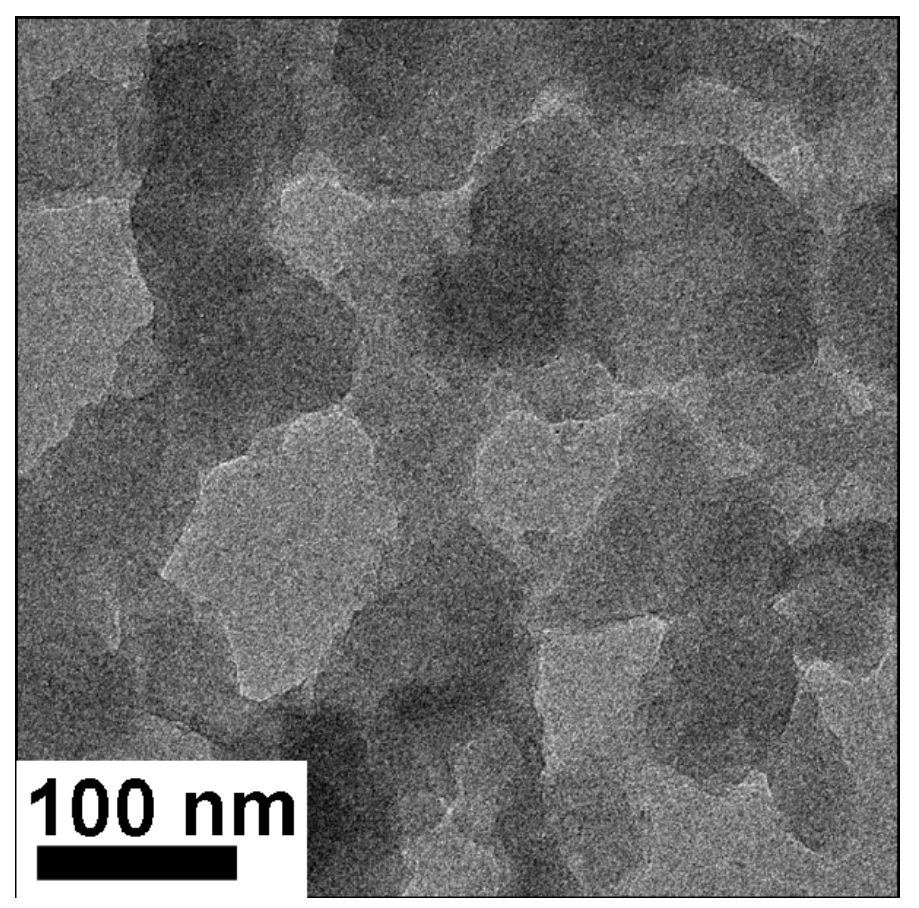

Figure 6.35 A TEM image shows randomly connected nanostructures with a contrast slightly higher than the background carbon support. 
The solid was studied under TEM. As may be seen in figure 6.35, the solid was found to be comprised of porous randomly-connected nanostructures. From SAED the nanostructures were found to be amorphous. EDS analysis revealed that the nanostructures were constituted of mainly phosphorus with a small amount of indium.

The result of this experiment indicates that no crystalline indium phosphide may be formed under these experimental conditions. Although heated in an oil bath at $200{ }^{\circ} \mathrm{C}$, the temperature of the reacting mixture was measured to be $\sim 170{ }^{\circ} \mathrm{C}$. A reaction temperature lower than $200{ }^{\circ} \mathrm{C}$ is likely to be the cause of the formation of an amorphous species of phosphorous instead of crystalline indium phosphide.

\subsection{A Mixture of Indium Trichloride and Phosphorus Pentabromide in Trioctylphosphine Treated with A Mixture of Lithium Borohydride and Indium Metal Nanoparticles (Experiment 8)}

An experiment was carried out adding a mixture of lithium borohydride and indium metal nanoparticles dropwise into a mixture of indium trichloride and phosphorus pentabromide in trioctylphosphine pre-heated to $250{ }^{\circ} \mathrm{C}$. A black solid was isolated after purification. The indium metal nanoparticles were of uniform diameter of $\sim 6 \mathrm{~nm}$ and were prepared as described in experiment 3 in chapter 5.
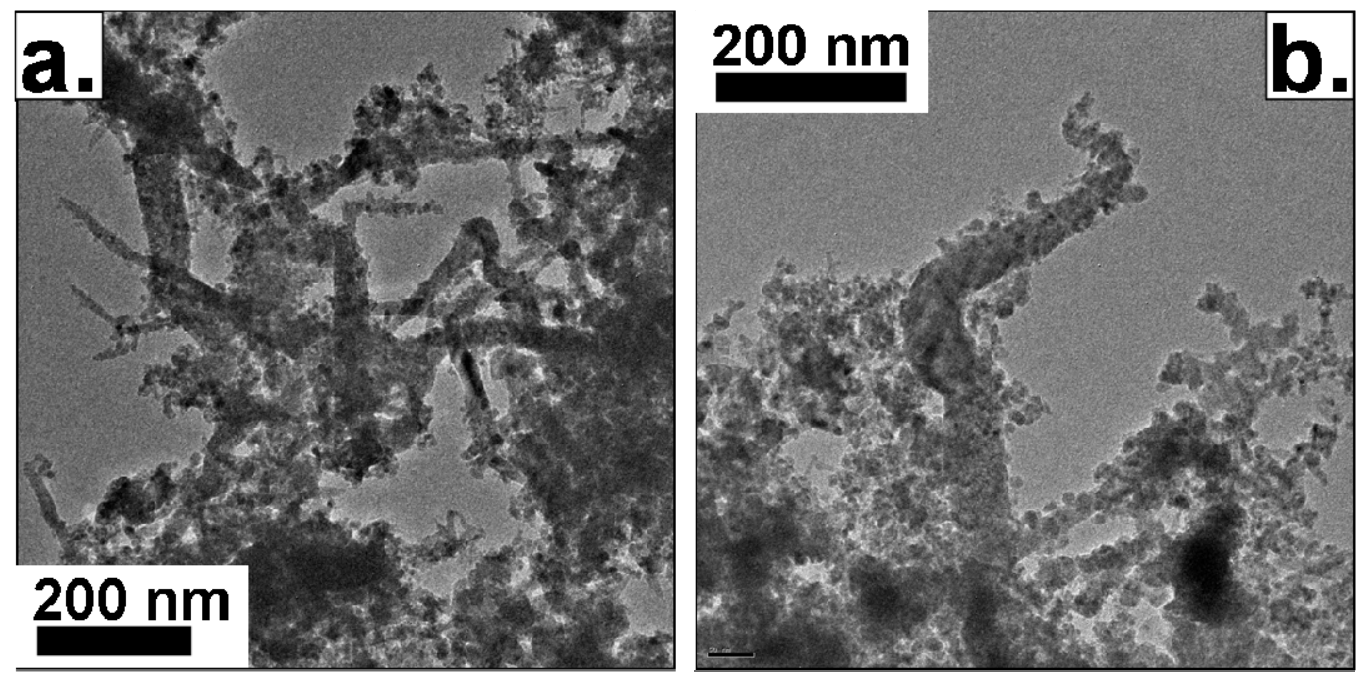

Figure 6.361 TEM images. (a) a mixture of nanowires decorated with nanoparticles and aggregated pieces of solid and (b) a nanowire with bends decorated and surrounded by nanoparticles. 
The solid was studied by TEM. As may be seen in figure $6.361 \mathrm{a}$ and $\mathrm{b}$ the solid was constituted of nanowires decorated with nanoparticles, aggregated particles and other irregularly-shaped nanostructures. From SAED, the nanostructures were found to be face centre cubic indium phosphide. Due to the aggregated nature of the nanostructures it is difficult to establish statistically meaningful size and shape distributions. Generally speaking, the nanowires have lengths $>200 \mathrm{~nm}$ and diameters ranging from $7 \mathrm{~nm}$ to 100 $\mathrm{nm}$. The aggregated particles have sizes that vary vastly depending on the degree of aggregation. Segregated nanoparticles of size smaller than $10 \mathrm{~nm}$ were also observed. Both perfect single crystals and crystals that contain defects were observed as may be seen in figure 6.362. Figure 6.363 a shows a crystalline nanowire.
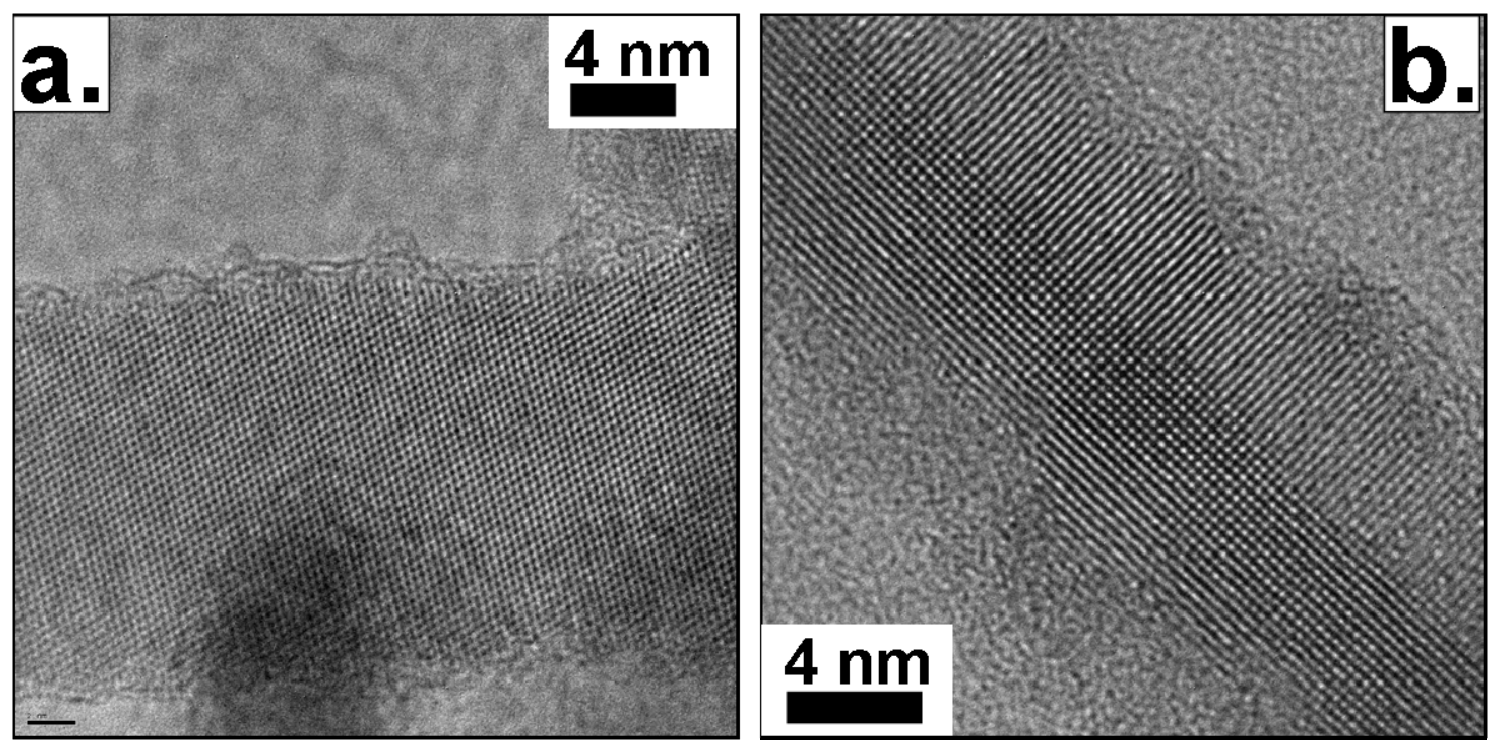

Figure 6.362 HRTEM images. (a) an indium phosphide nanowire without nanoparticles decoration and (b) an indium phosphide nanowire. 


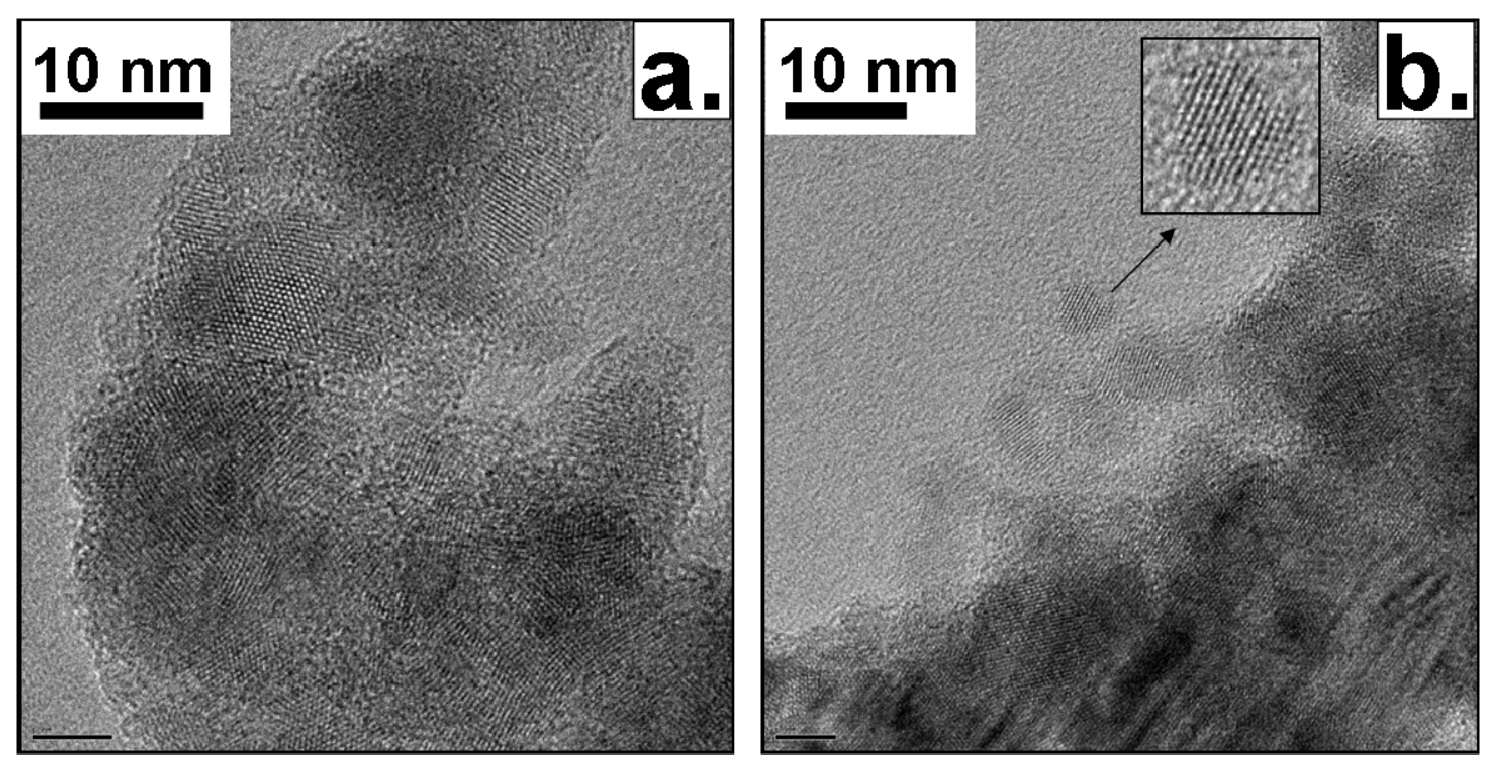

Figure 6.363 HRTEM images. (a) nanoparticles encapsulated in an amorphous layer of an uncharacterized material and (b) individual crystalline nanoparticles found alongside an aggregate.

As may be seen in figure 6.363 a, the aggregates contain crystalline nanoparticles encapsulated in an amorphous coating. The nature of the coating is unclear. It could be the result of partial oxidation of the aggregates or it could be of residual hydrogen phosphide. In figure $6.363 \mathrm{~b}$ segregated crystalline nanoparticles less than $10 \mathrm{~nm}$ in size could be observed as spheres with lighter contrasts. Lattice fringes may be seen. No amorphous layers were observed around the nanoparticles. The crystalline nanoparticles were believed to be face centred cubic indium phosphine because only face centred cubic indium phosphide was observed in the electron diffraction pattern of a mixture containing both the aggregated segregated nanoparticles

This experiment proved that crystalline indium phosphide nanowires and nanoparticles could be prepared from reacting a mixture indium trichloride and phosphorus pentabromide with a mixture of lithium borohydride and indium metal nanoparticles. Although nanowires were successfully prepared, they were not pure from nanoparticles and aggregated nanostructures. 


\subsection{Indium Trichloride in Trioctylphosphine Treated with Phosphorus Pentabromide and Lithium Borohydride (Experiment 9)}

An experiment was carried out adding solutions of phosphorus pentabromide and lithium borohydride slowly to a solution of indium trichloride in trioctylphosphine heated to $\sim 250{ }^{\circ} \mathrm{C}$. A fixed amount of lithium borohydride was first injected in order to create indium metal nanoparticles as seeds. Following the in situ formation of indium seeds, the solutions of phosphorus pentabromide and lithium borohydride were added dropwise from two separate syringes. The additions proceeded in a fashion such that a drop of phosphorus pentabromide solution would be added 10 seconds after a drop of lithium borohydride solution was added and vice versa. The alternating additions of phosphorus pentabromide and lithium borohydride were completed in 45 minutes. The resulting mixture was then reacted for another 2 hours. A deep brown to black solid was isolated after purification and was studied by TEM.

Figure 6.370 a showed the solid was constituted of mainly nanowires with a small amount of nanoparticles. The nanowires have an average length of $647 \pm 301 \mathrm{~nm}$ and an average diameter $48 \pm 22 \mathrm{~nm}$ and an average aspect ratio 16 \pm 10 . As shown in figure $6.370 \mathrm{c}$ the length distribution of the nanowires spread approximately evenly across the range of $<300 \mathrm{~nm}$ to $>900 \mathrm{~nm}$. Almost half of the population of the nanowires have a diameter in between $40-50 \mathrm{~nm}$ as may be seen in figure $6.370 \mathrm{~d}$. 

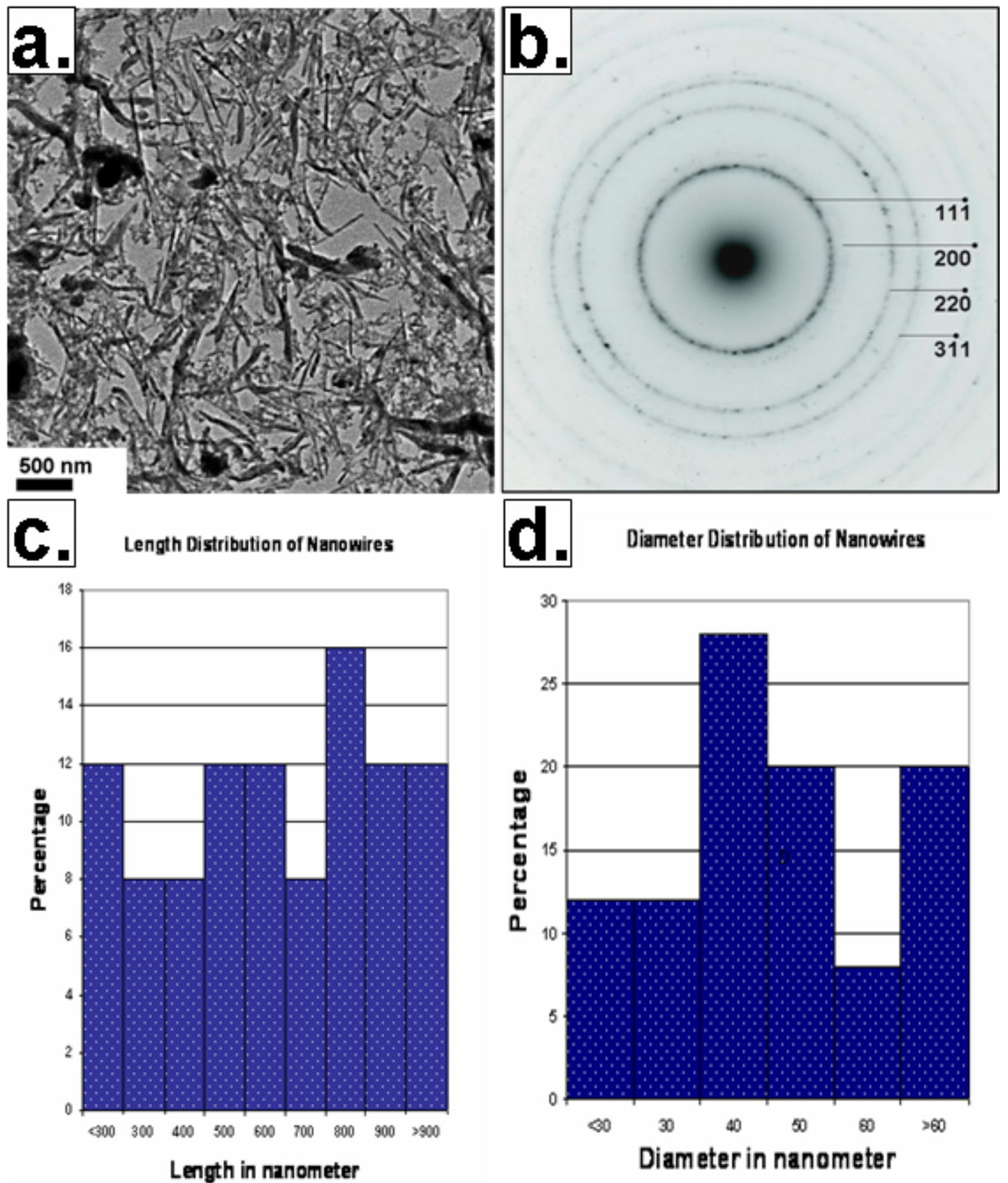

Figure 6.370 TEM analysis. (a) a TEM image of nanoparticles and indium phosphide nanowires; (b) a representative SAED diffraction pattern indexed to be face centred cubic indium phosphide; (c) length distribution of nanowires and (d) diameter distribution of nanowires. 


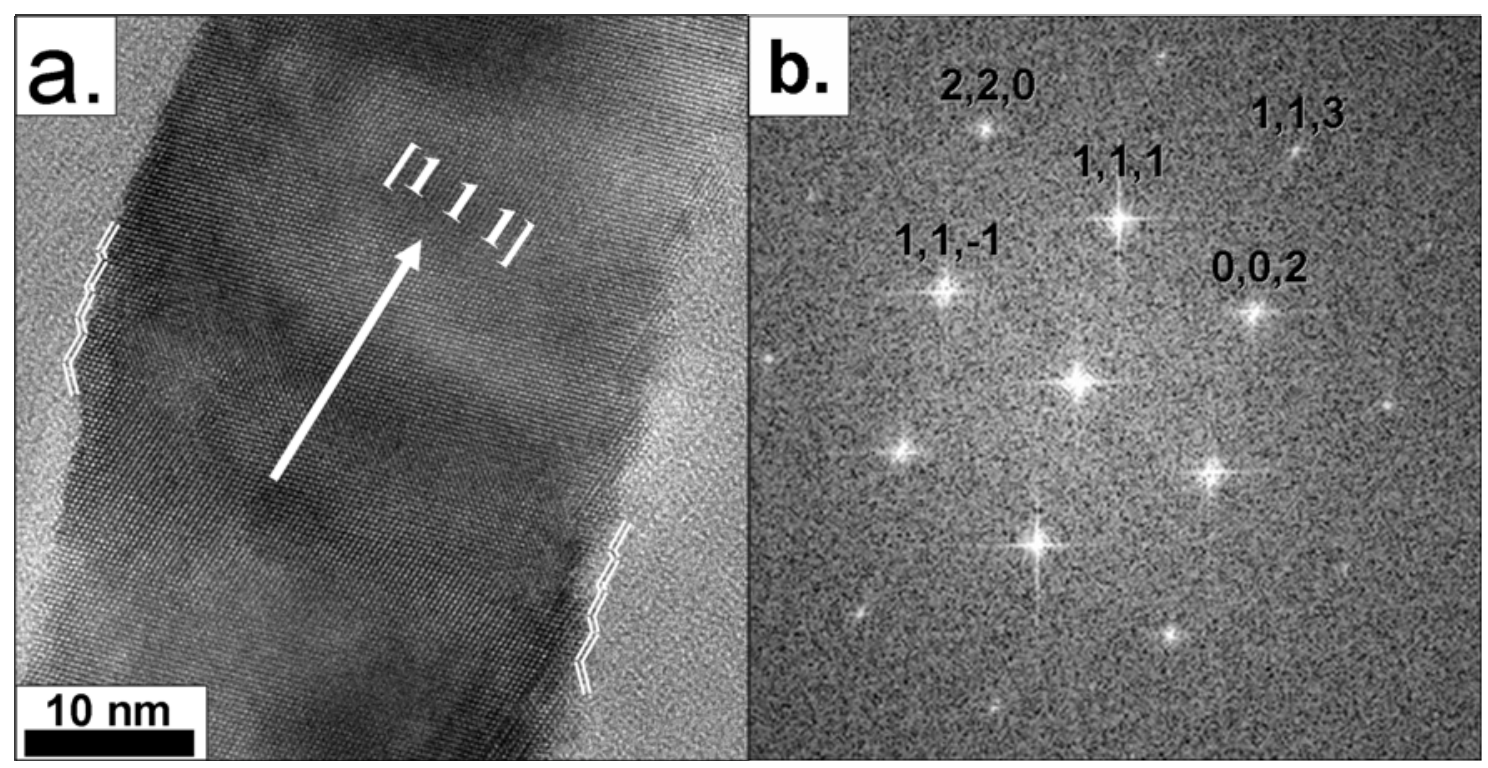

Figure 6.371 (a) a HRTEM image of a single crystalline indium phosphide nanowire growing on the [110] direction. Nanofacets on the edge of the naowire may be observed and (b) a power spectrum calculated from the image.

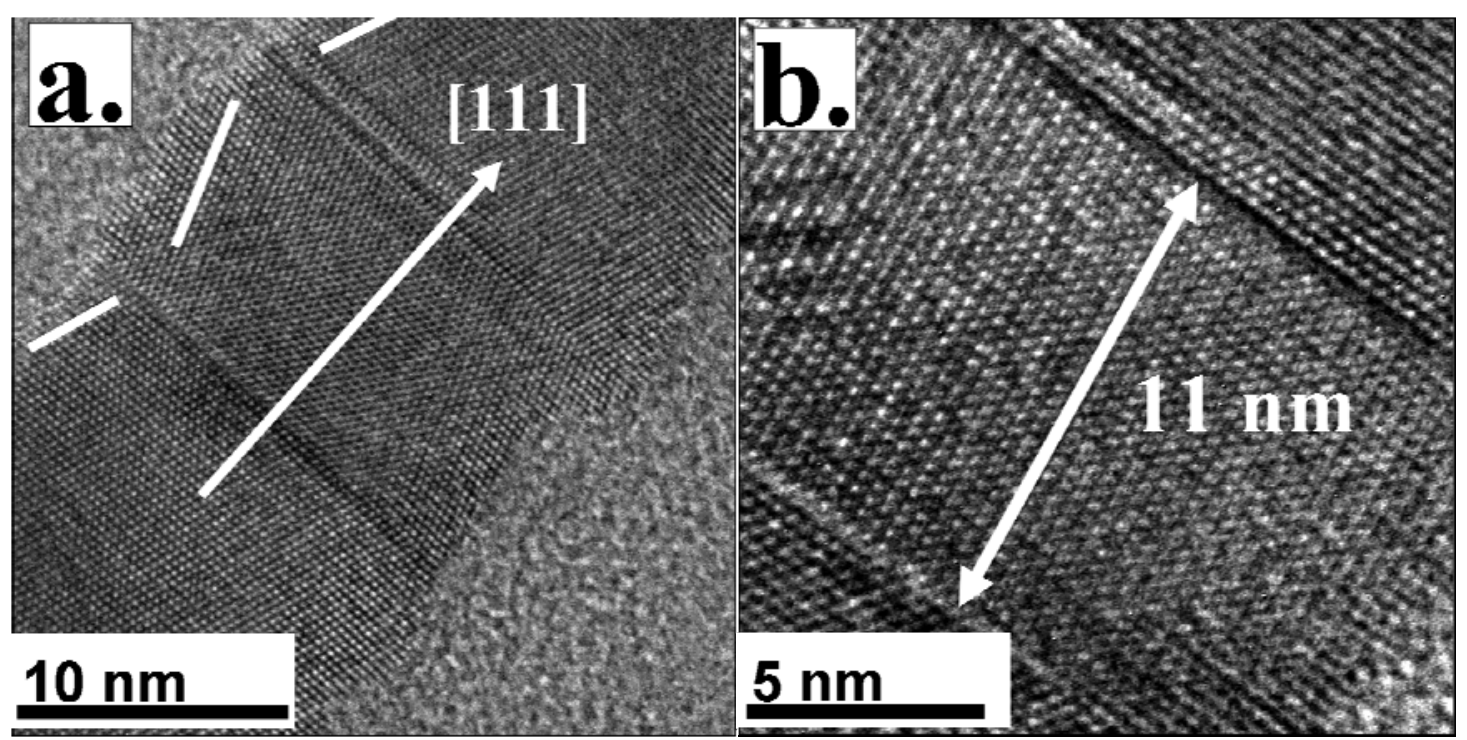

Figure 6.372 HRTEM images (a) an indium phosphide nanowire grown in [111] direction containing twin defects and (b) two successive twining boundaries $\sim 11 \mathrm{~nm}$ apart.

As may be seen in figure 6.371, a single crystalline nanowire growing in the [111] direction exhibits nanofacets on its edges. The growth direction of the nanowire was found by indexing the corresponding power spectrum simulated from the image. The difference in contrast across the nanowire indicate that nanowire was off the zone axis by a very a small degree. 
Figure 6.372 a showed an indium phosphide nanowire grown in the [111] direction. The growth direction was elucidated from indexing the corresponding diffraction presented as figure 6.373. From the literature, the [111] is the most common growth direction for cubic indium phosphide nanowires. ${ }^{10,11,15 b, 21}$

The nanowire is not single crystalline but contains twin boundaries normal to the axial growth direction of the nanowire (two dark lines perpendicular to the axial direction). Two successive twin boundaries were measured to be $\sim 11 \mathrm{~nm}$ apart (figure $6.372 \mathrm{~b}$ ).

A twinning boundary is isolated to a single atomic plane that separates two neighbouring crystal domains. The two domains are mirror images of each other if the twinning boundary is regarded as a mirror plane. The packing of atoms is still that of close-packing. The ABCABC packing in a perfect face centred cubic structure becomes ABC*BAC. $C^{*}$ represents the location of the reflection plane. ${ }^{22}$

The twins boundaries observed in figure 6.372 were characterized to be $\{111\}$ twins because the two neighbouring domains are of the $\{111\}$ family. $\{111\}$ twins form easily in face centred cubic (zinc blende) crystals ${ }^{23}$ such as gallium arsenide (GaAs), gallium phosphide (GaP), zinc selenide ( $\mathrm{ZnSe}$ ) and indium phosphide ${ }^{5,8,9,24-27}$ and it is difficult to limit their formation.

From the literature the occurrence of the $\{111\}$ twins is found to be related to several factors including (i) the local concentration of nuclei ${ }^{4}$; (ii) the local temperature during the growth of nanowires ${ }^{28}$; (iii) the contact angle between the metal seed and the semiconductor $^{22}$ and (iv) the nanowire diameter ${ }^{22}$.

In the case of VLS-grown gallium phosphide (GaP) nanowires seeded by gold nanoparticles, an oscillating modulation of the local semiconductor concentration was found to result in the occurrence of $\{111\}$ twining in a high frequency. ${ }^{4}$ It has also been found that in the case of GaAs nanowires that a growth temperature $60{ }^{\circ} \mathrm{C}$ lower than the initiation temperature helped eliminate the formation of twinning defects by suppressing the formation of twin nuclei. ${ }^{28}$ 
In the case of solution synthesis, the situation is further complicated by the interaction of surfactants and solvents with the nanowires during their growth. Davidson and coworkers proposed that the formation of twins could be related to the seed/nanowire contact angle. ${ }^{22}$ For gallium phosphide (GaP) and indium arsenide (InAs) nanowires grown from gold metal seeds, the fluctuations in the contact angles were found to result in the formation of $\{111\}$ twins. Davidson and coworkers have also found that nanowires with diameters smaller than $10 \mathrm{~nm}$ exhibit less twinning. ${ }^{25,26}$

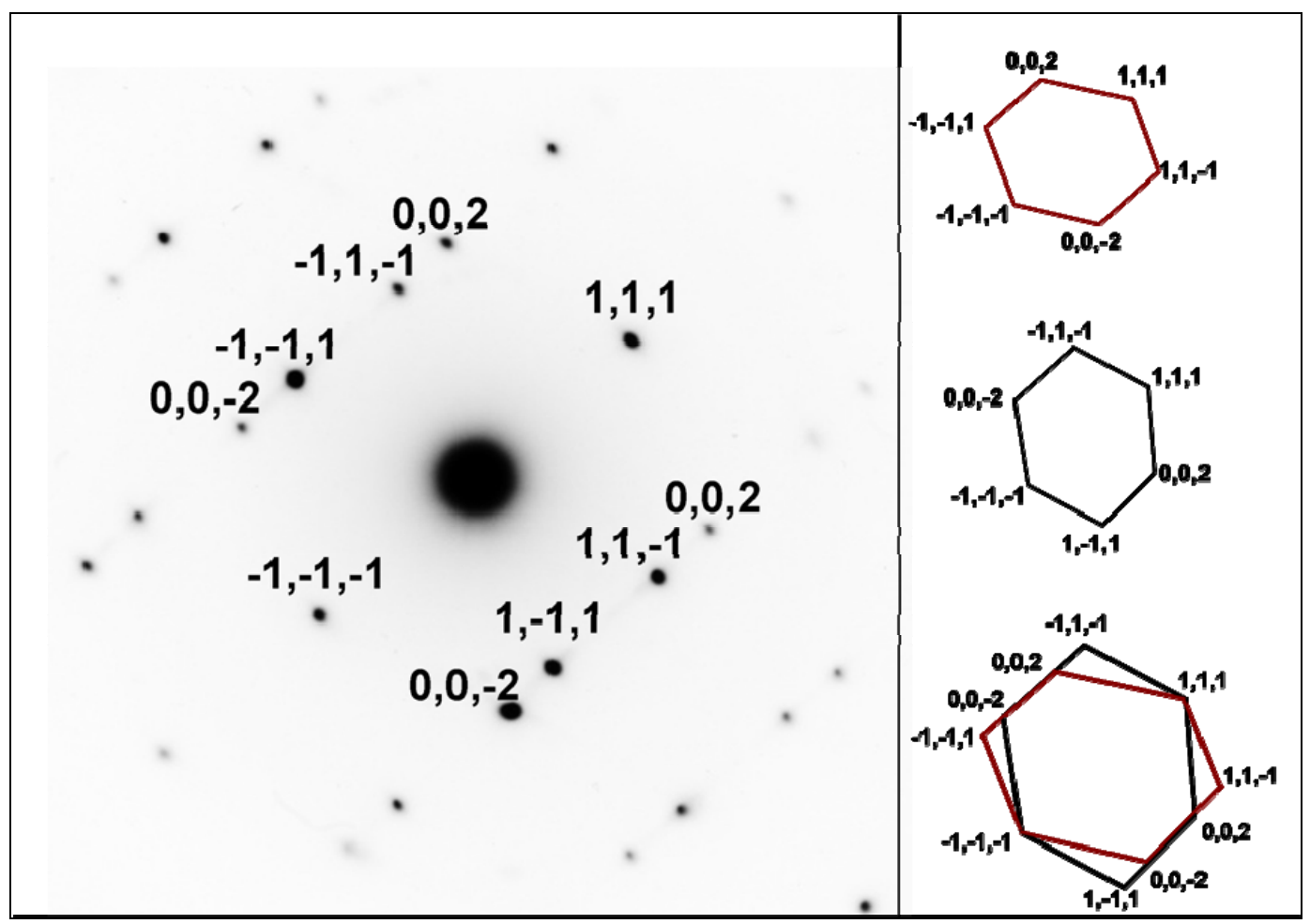

Figure 6.373 Electron diffraction pattern of the indium phosphide nanowire shown in figure 6.372 looking down the [110] direction. The diffraction pattern is a superimposition of two single crystal diffraction patterns which are mirror images of each other when reflected across the axial direction.

Figure 6.373 shows the diffraction pattern of the indium phosphide contained twin defects as shown in figure 6.372. The diffraction pattern was indexed. The pattern could be considered as a superimposed diffraction pattern from two single crystal diffraction patterns which are mirror images of each other. ${ }^{6}\{002\}$ diffractions observed were the results of double diffractions from pairs of $\{111\}$ planes present at the edge of the nanowire. $^{29}$ 


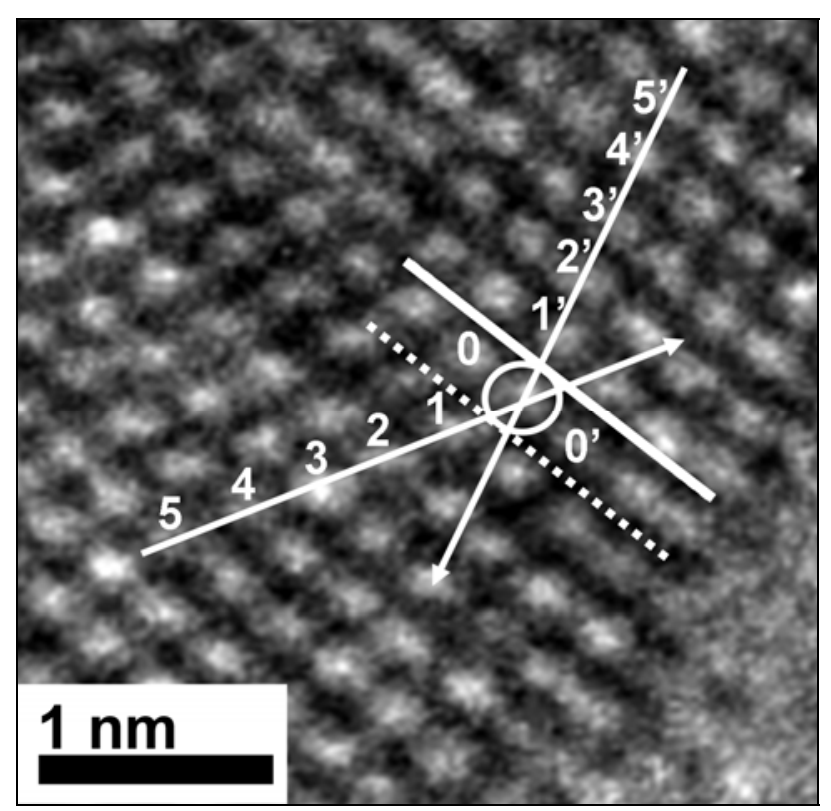

Figure 6.374 Analysis of twin defect. The twin was determined to occur across an atom of indium or phosphorous as circled.

There are four possible twinning arrangements: (i) InPInPInP-In-PInPInPIn (ii) PInPInPIn-P-InPInPInP (iii) InPInPInP-PInPInPIn and (iv) PInPInPIn-InPInPInP. In type (i) and (ii), the reflection plane coincides with a line of atoms of indium or phosphorous respectively. The occurrence of these twins would not result in any change in chemical composition. In type (iii) and (iv) the reflection plane lies "in-between” two adjacent atoms as represented by a white dashed line shown in figure 6.374. If twins of type (iii) and (iv) occurred they would result in a change in chemical composition.

A closer examination on one of the twin boundaries revealed thaat the twinning occurred across a line of atoms. As may be seen in figure 6.373, atoms 1-5 and 1'-5' are mirror images of each other if the plane in which atom $0\left(0^{\prime}\right)$ lies is regarded as a mirror. The two lines crossed at the circled atom marked as $0\left(0^{\prime}\right)$. Thus the twin is of either type (i) or type (ii).

This experiment proved that indium phosphide nanowires could be prepared from indium trichloride, phosphorous pentabromide and lithium borohydride. Under the synthetic conditions described in this experiment, indium phosphide were prepared primarily as nanowires. Both single crystal nanowires and nanowires with twin defects were observed. The nanowires characterized were found to grow along the [111] direction. 


\subsection{A Mixture of Indium Trichloride and Pre-synthesized \\ Indium Metal Seeds Treated with Phosphorus \\ Pentabromide and Lithium Borohydride \\ (Experiment 10)}

An experiment was carried out adding phosphorus pentabromide and lithium borohydride slowly to a mixture of indium trichloride and indium metal seeds in trioctylphosphine at $\sim 250{ }^{\circ} \mathrm{C}$. Phosphorus pentabromide and lithium borohydride were added separately and the additions were alternated with a period of $\sim 10$ seconds. The indium metal seeds were prepared as described in experiment 4 in chapter 5 . The indium metal nanoparticles were polydispersed in size and have diameter ranged between 10-150 nm. A deep brown to black solid was isolated after purification.

X-ray diffraction analysis showed that the solid was constituted of one single crystalline species: face centred cubic indium phosphide.

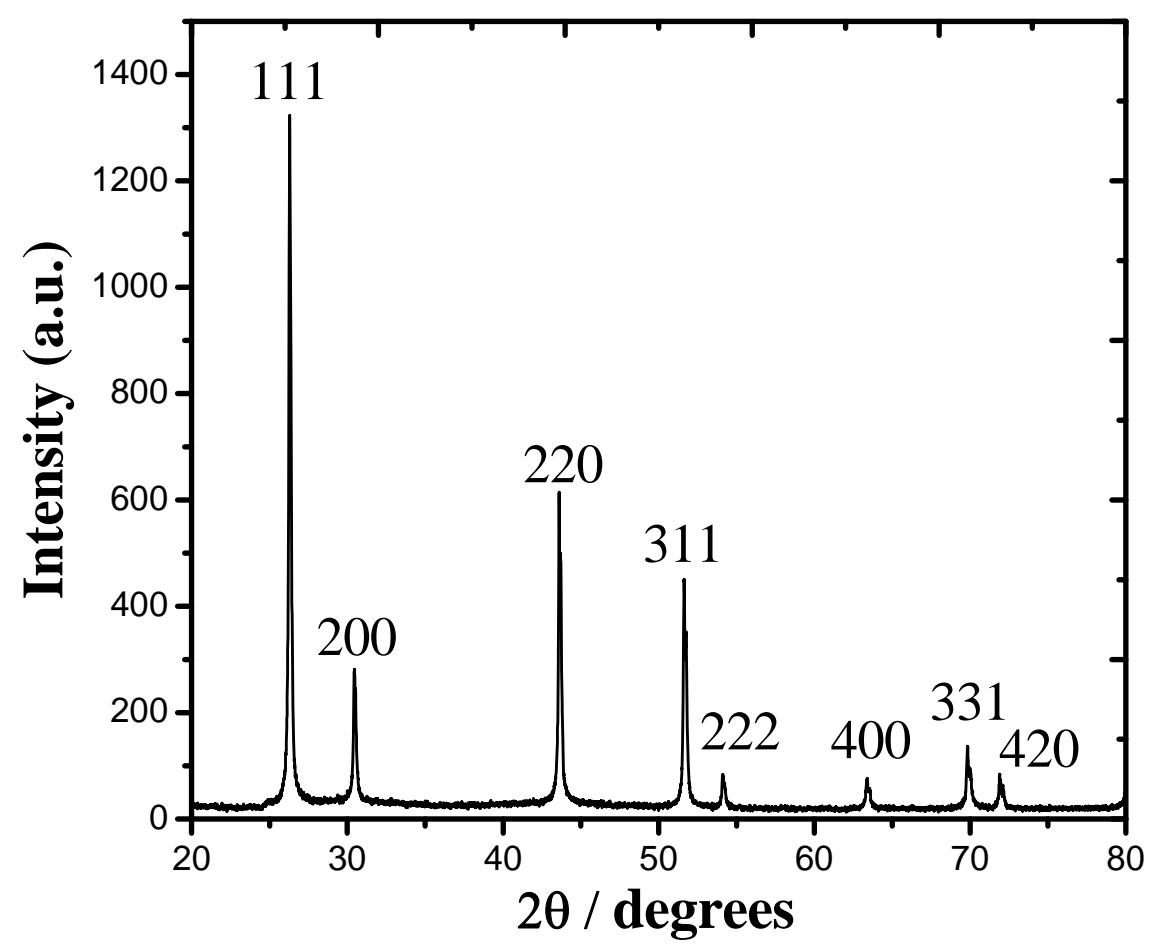

Figure 6.380 XRD pattern of indium phosphide nanowires after purification. The pattern corresponds to face centred cubic indium phosphide. 


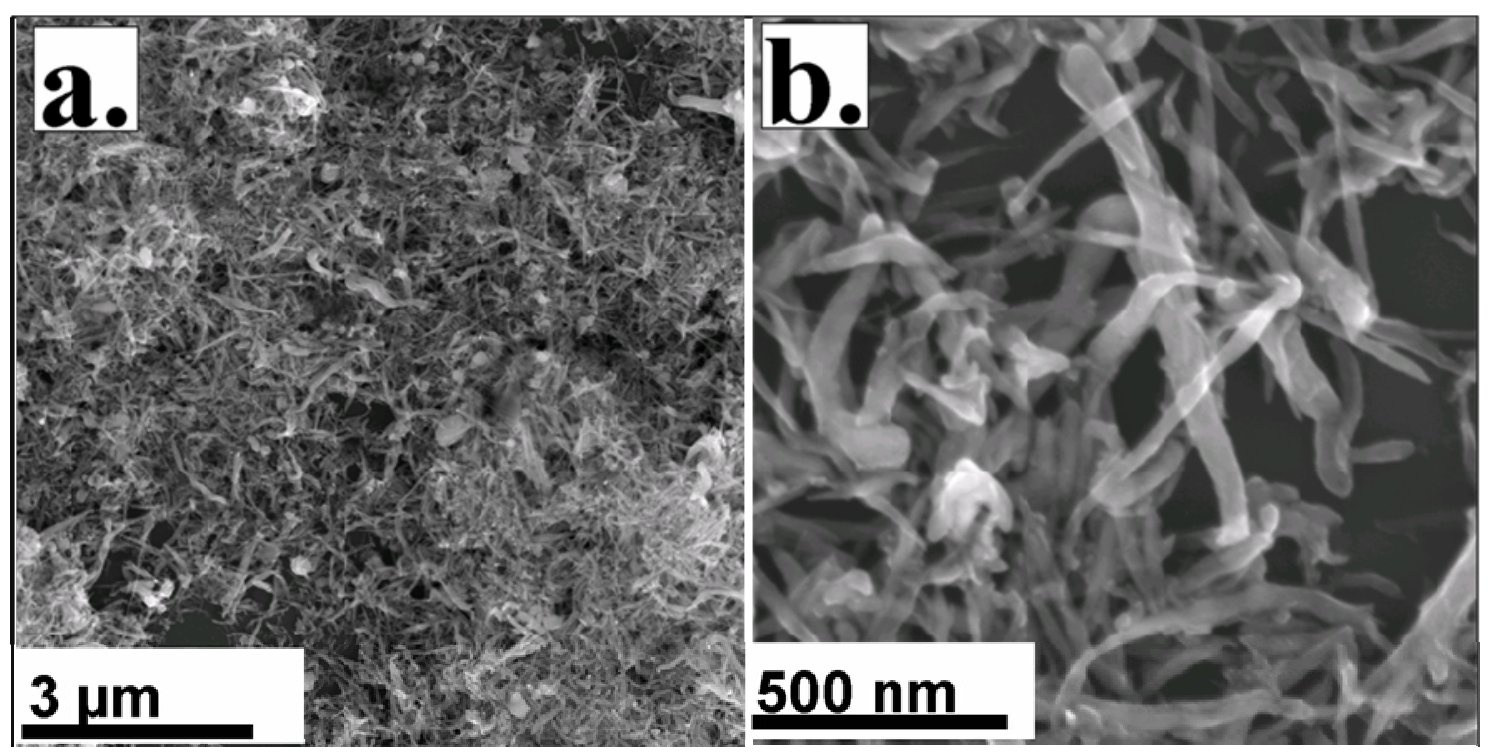

Figure 6.381 FESEM images of indium phosphide nanowires processed into a solid film by a drop-deposition method. (a) a mat of entangled indium phosphide nanowires and (b) close-up look of indium phosphide nanowires.
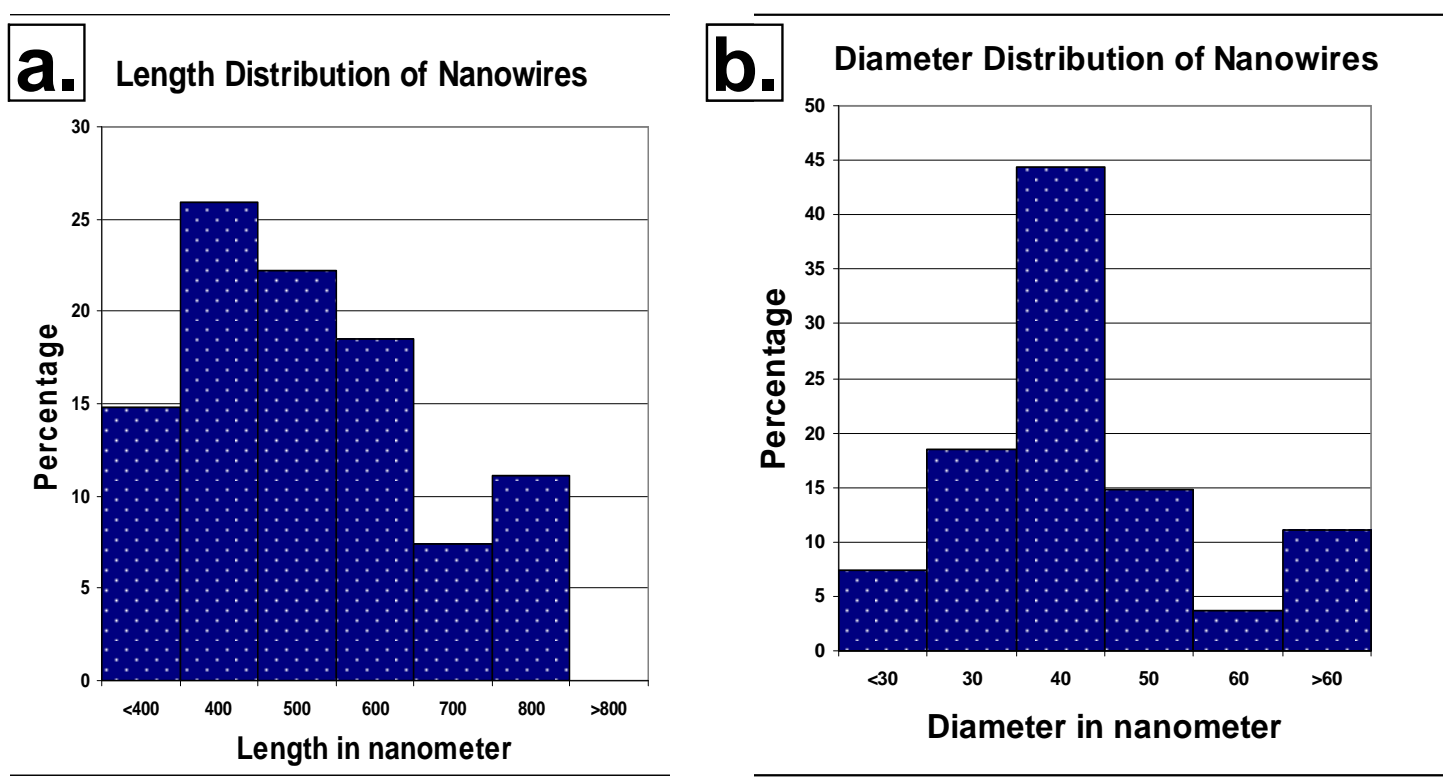

Figure 6.382 Histograms. (a) length distribution of indium phosphide nanowires and (b) diameter distribution of indium phosphide nanowires.

As may be seen in figure $6.381 \mathrm{a}$ and $\mathrm{b}$, the indium phosphide nanowires isolated formed a dense mat with nanowires entangled with each other when dried into films. The nanowires were moderately polydispersed in size with diameters ranging from 50$90 \mathrm{~nm}$ and lengths ranging from 200-800 nm. The nanowires were not perfectly straight. Bends and kinks were observed in many of the nanowires and many were found to taper towards the tip. The diameters of the nanowires were comparable to those of the indium 
metal seeds. The diameter distribution of the nanowires however was not as polydispersed as that of the indium metal nanoparticles. The difference may be explained by the theory of radial growth. ${ }^{30}$ During SLS growth, nuclei of a semiconductor which have crystallized out of the metal seed add to the growing crystal in the axial direction leading to the formation of a nanowire. Radial growth occurs when adatoms add directly to the surface of the growing nanowire resulting in the growth in the radial direction. However no direct evidence of radial growth was available.

The nanowires have an average length of $495 \pm 155 \mathrm{~nm}$, an average diameter of $42 \pm 13$ $\mathrm{nm}$ and an average aspect ratio of $13 \pm 5$. As may be seen in figure 6.382 , the length distribution is rather even across the range of $400 \mathrm{~nm}$ to $800 \mathrm{~nm}$. The diameter distribution is much centralized at $\sim 40 \mathrm{~nm}$.

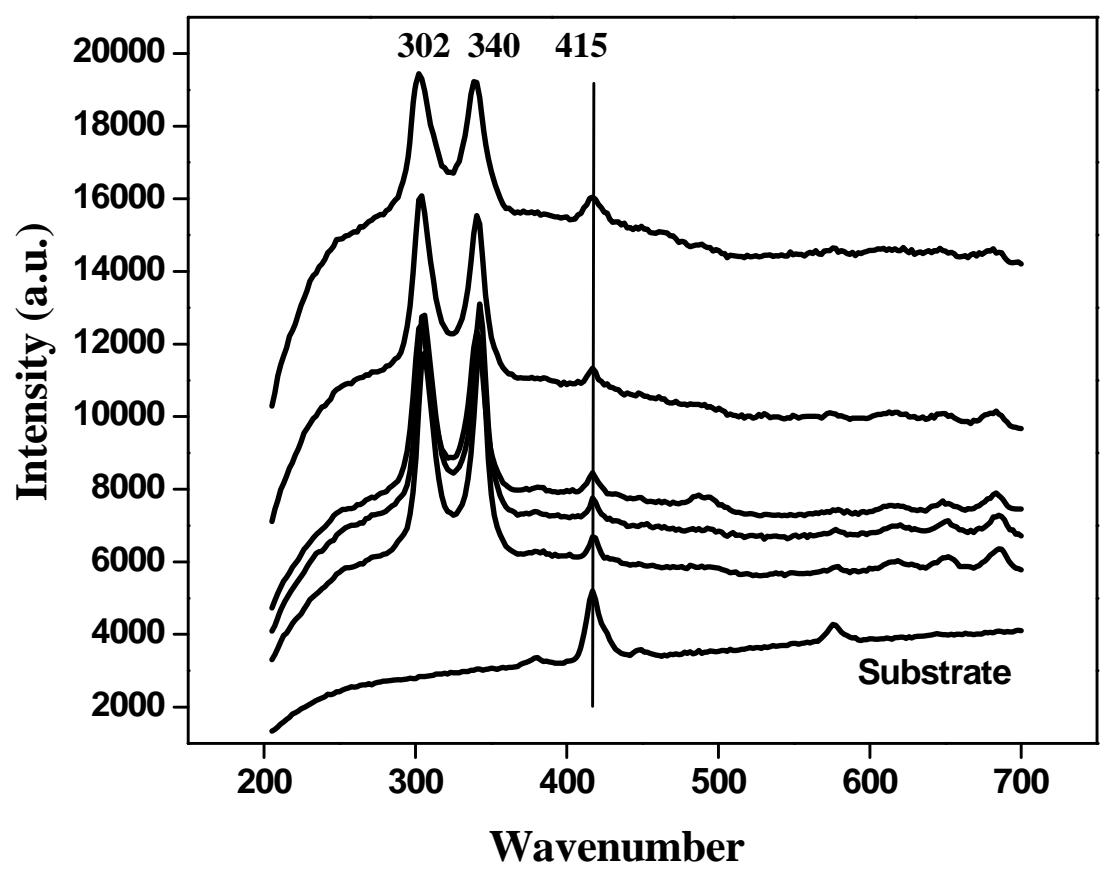

Figure 6.383 Raman spectra of indium phosphide nanowires prepared as a solid film.

Figure 6.383 is a collection of Raman spectra of the indium phosphide nanowires prepared. The signal at $302 \mathrm{~cm}^{-1}$ corresponds to the TO phonon and $340 \mathrm{~cm}^{-1}$ corresponds to the LO phonon. ${ }^{30}$ The signal observed at $415 \mathrm{~cm}^{-1}$ originated from the sapphire substrate. No indium oxide $\left(\mathrm{In}_{2} \mathrm{O}_{3}\right)$ was detected within the detection limit of the system. The Raman measurements were carried out $~ 2$ months after the nanowires were prepared. The Raman technique is sensitive to the surface composition. No signals 
due to crystalline indium oxide were observed, suggesting that the indium phosphide nanowires prepared did not oxidize over a period of 2 months.

\section{Optical Properties of Indium Phosphide Nanowires -Absorption and Photoluminescence}

Films of indium phosphide nanowires were prepared by slowly drying an indium phosphide nanowire solution on sapphire substrates in a confined environment. The film was subjected to absorption and photoluminescence studies at room temperature.
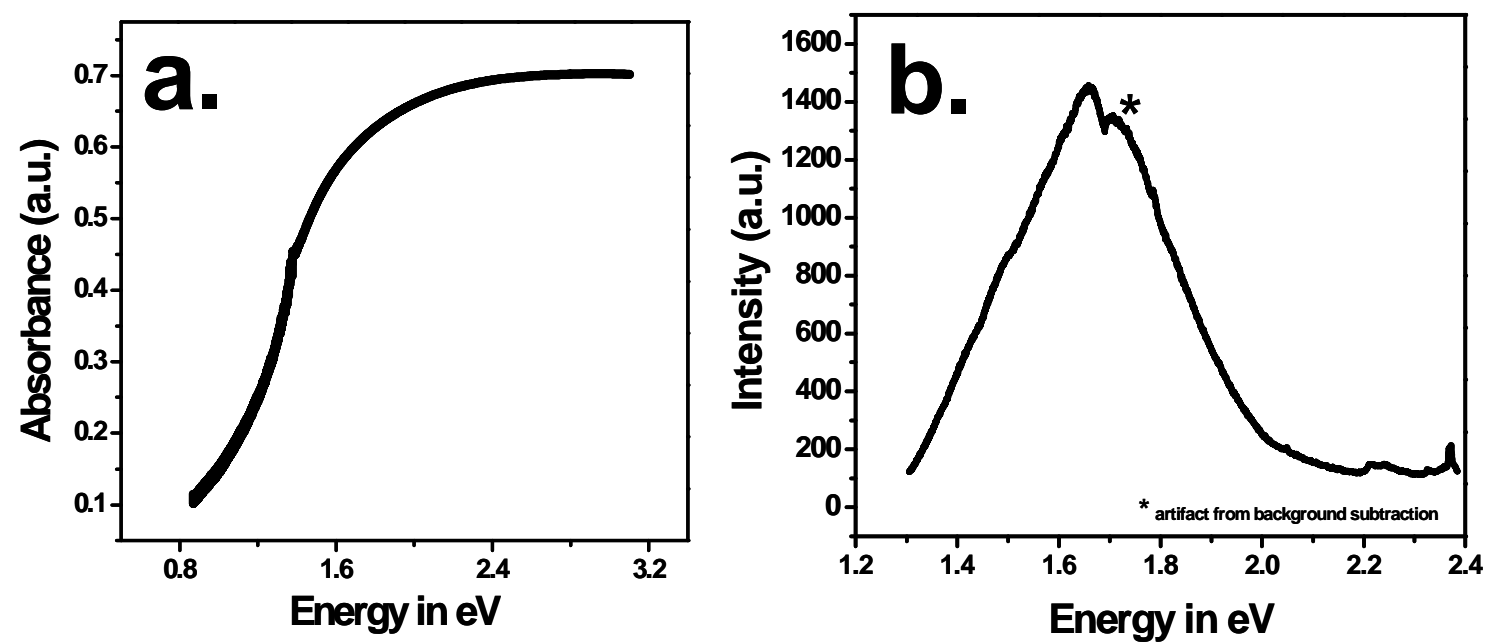

Figure 6.384 Optical properties of indium phosphide nanowires. (a) absorption edge at $1.35 \mathrm{eV}$ and (b) photoluminescence with an emission maxima at $1.66 \mathrm{eV}$.

An absorption edge at $1.35 \mathrm{eV}$ was observed as shown in figure $6.384 \mathrm{a}$. The indium phosphide nanowires exhibit photoluminescence at $293 \mathrm{~K}$ under illumination by an $\mathrm{Ar}^{+}$ laser of wavelength $514 \mathrm{~nm}$. The broad emission has an onset of $\sim 1.3 \mathrm{eV}$ and an emission maxima at $\sim 1.6 \mathrm{eV}$ (figure $6.384 \mathrm{~b}$ ). The feature marked with * was an artefact due to imperfect background subtraction. When compared to bulk indium phosphide which has a direct band gap of $1.34 \mathrm{eV}$ at $300 \mathrm{~K}$, the photoluminescence is blueshifted. $^{31}$ The blue shift is unlikely to be the result of quantum confinement effects, since the majority of the indium phosphide nanowires have diameters significantly larger than the excitonic Bohr radius ( $20 \mathrm{~nm})$ and may originate from surface state emission. $^{9,20}$ 
This experiment proved that indium phosphide could be prepared exclusively as nanowires from indium trichloride, phosphorous pentabromide and lithium borohydride in in the presence of pre-synthesized indium metal nanoparticles. The nanowires were found to exhibit photoluminescence at $1.6 \mathrm{eV}$ blued shift by $0.36 \mathrm{eV}$ when compared to the bulk band gap of indium phosphide of $1.34 \mathrm{eV}$.

\subsection{Indium Trichloride in Trioctylphosphine Treated with A Mixture of Bismuth Metal Nanoparticles, Hydrogen Phosphide and Lithium Borohydride (Experiment 11)}

Bismuth metal nanoparticles, hydrogen phosphide and lithium borohydride were mixed in N-N-diethylaniline. The solution was divided into seven equal portions. Each portion was injected into a solution of indium trichloride in trioctylphosphine every 3 minutes. The bismuth nanoparticles have an average diameter of $\sim 20 \mathrm{~nm}$ and were prepared as described in experiment 5 in chapter 5 . A black solid was isolated after purification.

The solid was studied under TEM. As may be seen in figure $6.39 \mathrm{a}$, the solid was constituted of nanowires and nanoparticles. The nanoparticles were found to be residual bismuth seeds. To remove the bismuth residue, the solid was treated with diluted hydrochloric acid in methanol. As may be seen in figure $6.39 \mathrm{~b}$, the resultant nanowires were free from other impurities. From SAED, the nanowires were found to be face centred cubic indium phosphide.

The diameters of the nanowires were close to being monodispersed. Apart from long straight nanowires (figure 6.39 a, b and d) nanowires with bents were also observed. Straight nanowires have an average diameter of $67 \pm 5 \mathrm{~nm}$ and their lengths vary between 940-3200 nm. An underestimated average aspect ratio of $27 \pm 18$ was calculated. The aspect ratio is underestimated because the full lengths of most of the nanowires were not caught in their TEM images and the lengths observed. The bent nanowires, by comparison, had larger diameters in the $200 \mathrm{~nm}$ range. 

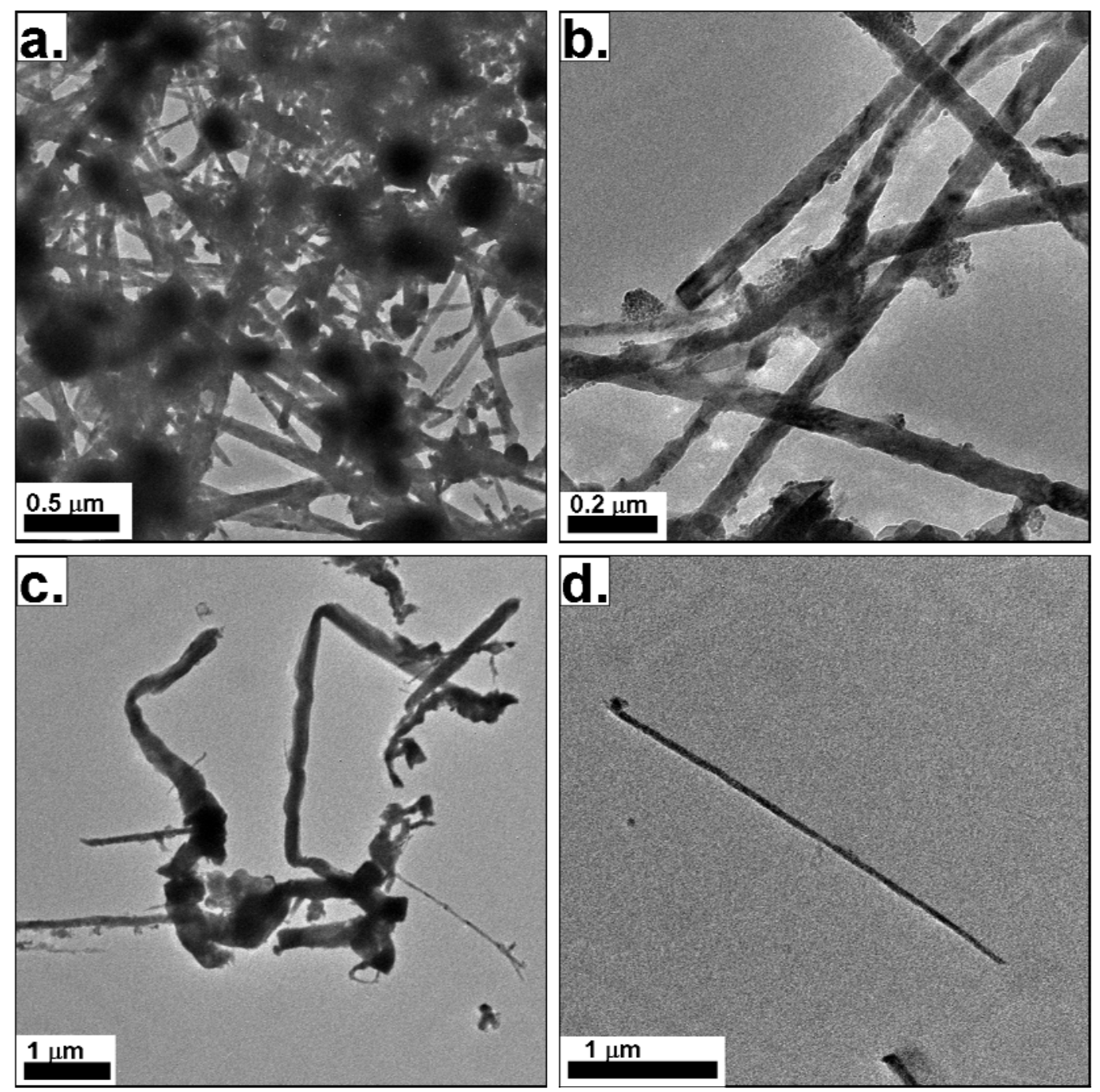

Figure 6.39 TEM images. (a) a group of nanowires with bismuth metal residues; (b) indium phosphide nanowires after removal of impurities; (c) indium phosphide nanowires with high degree of bend and kinks (d) a single straight nanowire of length up to $3 \mu \mathrm{m}$.

This experiment proved that indium phosphide nanowires could be prepared from indium trichloride, phosphorous pentabromide and lithium borohydride using bismuth metal as seeds. The nanowires have similar diameters and most of the nanowires are straight in morphology. 


\subsection{Discussion}

\section{Reactions of Indium Trichloride and Phosphorus}

\section{Pentabromide and Lithium Borohydride (Experiments 3-11)}
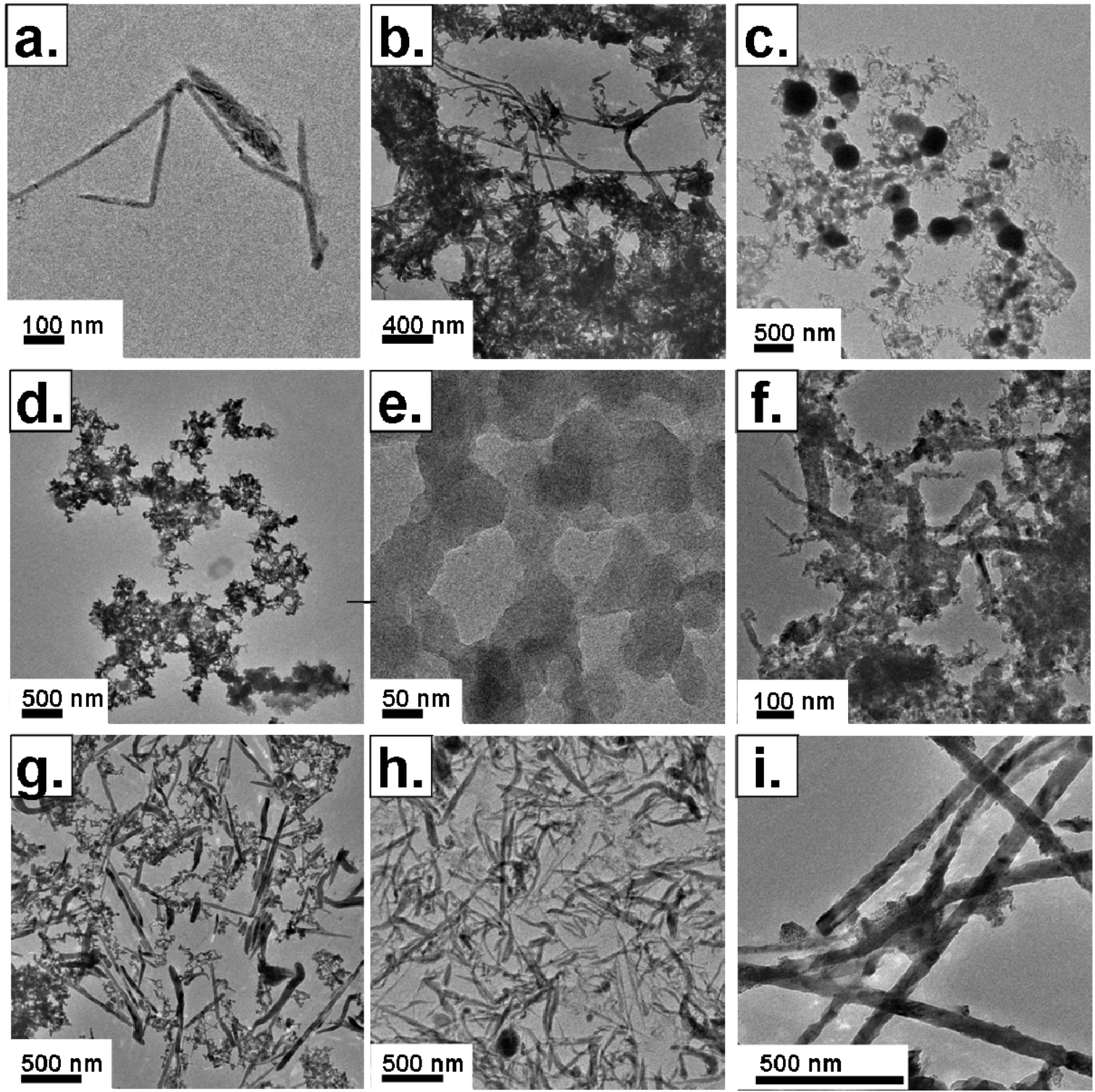

Figure 6.4 TEM images of products isolated from (a) experiment 3; (b) experiment 4;

(c) experiment 5; (d) experiment 6; (e) experiment 7; (f) experiment 8; (g) experiment 9;

(h) experiment 10 and (i) experiment 11. The best products in terms of indium phosphide nanowires were from (g) and (h) and (i). 
Figure 6.4 shows TEM images of products obtained from experiments 3-11. Indium phosphide nanowires were first observed in experiment 3. The method of preparation has since been improved. Although not all experiments present in figure 6.4 were directly comparable, the figure showed the "evolution" or "development" of the method of preparation based on the corresponding products observed.

\section{The Effect of Solvent: Comparisons of Experiment 4 with Experiment 6}

Trioctylphosphine, N,N-diethylaniline and tetraethyleneglycol have been tried as reaction solvents for the synthesis of indium phosphide nanowires. Amongst the three, trioctylphosphine was found to be the best in that segregated nanowires could be produced under the right conditions. The product isolated from a reaction carried out in tetraethyleneglycol comprised of amorphous nanoparticles only (not shown) without any crystals and nanowires. It was therefore considered an inappropriate solvent.

\begin{tabular}{|c|c|c|c|}
\hline Experiment & Solvent & Product & Figures \\
\hline 4 & Trioctylphosphine & $\begin{array}{c}\text { Segregated nanorods, } \\
\text { nanowires and nanofibres }\end{array}$ & $6.4 \mathrm{~b}$ \\
\hline 6 & N,N-diethylaniline & $\begin{array}{c}\text { Aggregated nanorods with } \\
\text { a high degree of bend }\end{array}$ & $6.4 \mathrm{~d}$ \\
\hline
\end{tabular}

Experiment 4 and experiment 6 involved the hot injection of hydrogen phosphide into a solution of indium trichloride in trioctylphosphine and in $\mathrm{N}, \mathrm{N}$-diethylaniline respectively. $\mathrm{N}, \mathrm{N}$-diethylaniline was found to cause a high degree of bend in nanorods when used as a solvent. However, due to N,N-diethylaniline was the only solvent found capable of dissolving hydrogen phosphide, a minimum amount of $\mathrm{N}, \mathrm{N}$-diethylaniline ( $\sim 2 \mathrm{~mL}$ ) was often used for transferring hydrogen phosphide to the reaction solvent ( $10 \mathrm{~mL}$ ). The reason behind that bent nanorods were formed in N,N-diethylaniline is unclear. A possible explanation is that $\mathrm{N}, \mathrm{N}$-diethylaniline is not sufficiently bulky to effectively prevent the aggregation of indium metal seeds out of which indium phosphide nanorods were growing. From the prospective of steric bulkiness, trioctylphosphine, which is bulkier than N,N-diethylaniline, is expected to prevent the aggregation of indium metal seeds much more effectively therefore relatively straight indium phosphide nanorods, nanowires were produced. 


\subsection{Hydrogen Phosphide: In Situ Formation versus Pre- Synthesized: Comparisons of Experiment 4 with Experiment 9}

In experiment 4 hydrogen phosphide was pre-synthesized whereas in experiment 9 hydrogen phosphide was formed in situ from every drop of phosphorus pentabromide added. The reaction of pre-formed hydrogen phosphide with indium trichloride and lithium borohydride in experiment 4 produced large nanofibers whereas the reaction of hydrogen phosphide formed in situ produced only nanowires.

\begin{tabular}{|c|c|c|c|}
\hline Experiment & Hydrogen Phosphide & Products & Figure \\
\hline 4 & Pre-formed & Nanorods, nanowires and & $6.4 \mathrm{~b} /$ \\
& & nanofibres & 6.32 \\
\hline 9 & Formed in situ & Nanowires & $6.4 \mathrm{~g}$ \\
\hline
\end{tabular}

There are two possible explanations to the formation of large nanofibres in experiment 4:

(i) the nanofibres formed from large nanoparticles of hydrogen phosphide (as observed in experiment 1). No nanofibres formed in experiment 9 because the size of hydrogen phosphide present was relatively small.

(ii) the nanofibres were formed as a result of an Ostwald ripening process. Ostwald ripening occurs when large particles grow at the expense of small nanoparticles. Small nanoparticles dissolve into the solvent to form nuclei that add on to large nanoparticles. ${ }^{32}$ Thus two groups of nanoparticles with distinctly different sizes form.

The first explanation is preferred for there is experimental evidence of the presence of large nanoparticles of hydrogen phosphide. As aforementioned, N,N-diethylaniline was the only solvent found during this research capable of dissolving hydrogen phosphide but it was also found to result in a high degree of bend in nanowires. Therefore a minimum amount of $\mathrm{N}, \mathrm{N}$-diethylaniline $(\sim 2 \mathrm{~mL})$ was often used for transferring hydrogen phosphide. Not all hydrogen phosphide could be dissolved in the minimum amount of $\mathrm{N}, \mathrm{N}$-diethylaniline and a dispersion of hydrogen phosphide in $\mathrm{N}, \mathrm{N}$ - 
diethylaniline was gained. The dispersion was expected to contain large nanoparticles of hydrogen phosphide which could react with indium metal to form large indium phosphide nanofibres.

\section{The effect of metal seeds}

\section{Indium Metal Seeds: In Situ Formation versus Pre- Synthesized (Comparisons of Experiment 9 with Experiment 10)}

Experiment 9 and 10 were conducted under similar conditions. The seeds used in experiment 9 were prepared in situ by adding a small amount of lithium borohydride into a preheated solution of indium trichloride in trioctylphosphine at the beginning of the reaction. In experiment 10, indium metal seeds employed were pre-synthesized. Although both experiments gave indium phosphide nanowires, the differences include (i) a significant amount nanoparticles were observed in experiment 9 and (ii) nanowires formed in experiment 9 have an average diameter smaller than that formed in experiment 10 .

\begin{tabular}{|c|c|c|c|}
\hline Experiment & Indium seeds & Products & Figure \\
\hline 9 & In situ & Nanowires and nanoparticles & $6.4 \mathrm{~g}$ \\
\hline 10 & Pre-synthesized & Nanowires only & $6.4 \mathrm{~h}$ \\
\hline
\end{tabular}

It is believed that in experiment 10 the formation of nanoparticles was suppressed resulting in almost exclusively nanowires. The amount of metal nanoparticles observed in experiment 9 was not determined due to experimental difficulty. The concentrations of indium metal seeds present in both experiments before the reactions started may not be the same and can not be compared. It is likely that the concentration of indium nanoparticles present in experiment 10 were many folds higher than that of experiment 9. The indium metal nanoparticles served as preferential sites to dissolve indium and phosphorous species therefore secondary nucleation was sufficiently suppressed. In experiment 9, the indium metal seeds formed in situ may not be of sufficient number to effectively suppress secondary nucleation process. 
The difference in nanowire diameters between experiment 9 and 10 suggested that the metal seeds formed in situ in experiment 9 were smaller in diameter than that of the preformed nanoparticles used in experiment 10. However, the size of the metal seeds formed in experiment 9 was not determined therefore it remained a speculation. It is well-documented that SLS grown nanowires often have diameter smaller but close to the metal nanoparticle seeds from which they grew.

\section{Bismuth Metal Nanoparticles versus Indium Metal Nanoparticles: Comparisons of Experiment 10 with Experiment 11}

Both experiment 10 and experiment 11 involved the use of pre-synthesized metal nanoparticles as seeds. In experiment 10 pre-synthesized indium metal nanoparticles were used whereas in experiment 11 pre-synthesized bismuth seeds were used. The two experiments differed mainly in that: (i) in experiment 10 hydrogen phosphide was prepared in situ during the dropwise addition of phosphorous pentabromide and lithium borohydride whereas in experiment 11 hydrogen phosphide was pre-synthesized in N,N-diethylaniline; (ii) multiple injections of a mixture of hydrogen phosphide and lithium borohydride were performed in experiment 11 whereas in experiment 10 hydrogen phosphide and indium metal were continuously formed.

\begin{tabular}{|c|c|c|c|}
\hline Experiment & $\begin{array}{c}\text { Pre-synthesized } \\
\text { seeds }\end{array}$ & Products & Figure \\
\hline 10 & Indium & $\begin{array}{c}\text { Nanowires with bend and } \\
\text { twists }\end{array}$ & $6.4 \mathrm{~h}$ \\
\hline 11 & Bismuth & Straight nanowires & $6.4 \mathrm{i}$ \\
\hline
\end{tabular}

The nanowires prepared from bismuth seeds when compared to that prepared from indium seeds have (i) a larger aspect ratio (ii) straighter edges (iii) a tighter diameter distribution. Bismuth nanoparticles have been found to be a better choice when compared to indium and tin ${ }^{15 b}$ in literature although the reasons behind are unclear. When compared to indium metal seeds, bismuth metal seeds are expected to have a higher melting point. The solubilities of indium and phosphorous in bismuth are also expected to be lower and that bismuth metal seeds are less likely to form phosphide. A 
combination of these three factors are likely to be the reason behind the fact that straighter indium phosphide nanowires were produced from bismuth seeds.

It is unclear if multiple injections would lead to better diameter distribution. Some previous work on multiple injections of phosphorous pentabromide and lithium borohydride to indium trichloride seeded by indium metal seeds produced large indium phosphide crystals instead nanowires. It is believed that a slow but continuous generation of hydrogen phosphide and indium metal during the reaction would help produce indium phosphide nanowires with uniform diameter and length via a diffusioncontrol growth process.

\section{The Effect of Reaction Temperature: Comparisons of Experiment 3 with Experiment 7}

Experiment 3 and experiment 7 both involved heating quickly a mixture of phosphorous pentabromide, lithium borohydride and indium trichloride. In experiment 3 the heating was executed by injecting the mixture into a hot trioctylphosphine solution at $250{ }^{\circ} \mathrm{C}$.

\begin{tabular}{|c|c|c|c|}
\hline \multirow{2}{*}{ Experiment } & $\begin{array}{r}\text { Reaction Temperature } \\
\text { in }{ }^{\mathbf{0}} \mathbf{C}\end{array}$ & Products & Figure \\
\hline 3 & 250 & Nanowires & $6.4 \mathrm{a}$ \\
\hline 7 & 170 & Amorphous, porous solid & $6.4 \mathrm{e}$ \\
\hline
\end{tabular}

In experiment 7, the mixture was heated in an oil bath pre-heated to $\sim 200{ }^{\circ} \mathrm{C}$ and due to a temperature gradient the actual reaction temperature of the mixture was measured to be only $170{ }^{\circ} \mathrm{C}$. While crystalline indium phosphide nanowires formed in experiment 3 , only amorphous random networks of a phosphorous containing material were obtained in experiment 7. A reaction temperature higher than $170{ }^{\circ} \mathrm{C}$ would be necessary for the formation of crystalline indium phosphide nanowires from such systems. 


\subsection{Conclusions}

A new method was developed to prepare highly crystalline indium phosphide nanowires in solution from indium trichloride and phosphorus pentabromide in the presence of lithium borohydride. Indium trichloride and phosphorus pentabromide were reduced by lithium borohydride to form indium metal and hydrogen phosphide respectively. Indium metal and hydrogen phosphide then reacted to form indium phosphide.

The method was unprecedented in (i) the use of phosphorus pentabromide as the phosphorus source and (ii) the preparation of hydrogen phosphide from phosphorus pentabromide and lithium borohydride in hexadecane and (iii) the preparation of indium phosphide nanowires from indium trichloride, phosphorus pentabromide and lithium borohydride.

Two sets of optimized conditions were developed for the synthesis of indium phosphide nanowires :

Set (i)

1. To add phosphorus pentabromide and lithium borohydride into indium trichloride in a slow controlled fashion. Such an approach was better than a direct injection in that indium phosphide nanowires were formed as the main product and in a high yield.

2. To use pre-synthesized indium metal nanoparticles as seeds.

Set (ii)

1. To prepare hydrogen phosphide in N,N-diethylaniline instead of in hexadecane.

2. To use bismuth metal nanoparticles as seeds.

The nanowires synthesized consisted of single crystals and nanowires that contain $\{111\}$ twin defects and nanofacets as a result of the twinning. Optical studies on solid films prepared from the indium phosphide nanowires showed that samples exhibit photoluminescence with an emission maxima at $1.65 \mathrm{eV}$ at $293 \mathrm{~K}$, blue shifted with regard to the bulk indium phosphide. 


\subsection{Experimental}

\section{Materials and methods}

\section{Chemicals}

All chemicals used in the synthesis reported in this chapter were of analytical grade. Air and moisture sensitive chemicals were stored under nitrogen in Schlenk round bottom flasks before use. All solvents and surfactants used in the reactions have boiling points higher than $200{ }^{\circ} \mathrm{C}$ were heated to reflux under vacuum for at least an hour before reaction. Refluxing under vacuum served to remove air and residue water and any impurities (of lower boiling points) that may be present. All reactions were conducted in nitrogen atmosphere unless otherwise stated.

\section{Synthesis of InP nanowires (Experiment 1)}

\section{Solution 1}

A mixture containing $1.002 \mathrm{~g}$ tetraoctylammonium bromide and $50 \mathrm{~mL}$ of hexadecane was heated to $120{ }^{\circ} \mathrm{C}$ under vacuum for 2 hours to form a cloudy white solution. To the solution at $48{ }^{\circ} \mathrm{C}$ was added $20 \mathrm{~mL}$ of toluene under nitrogen. The resulting mixture was heated under nitrogen to $150{ }^{\circ} \mathrm{C}$ to form a transparent colourless solution. The clear solution was cooled to room temperature with constant stirring.

\section{Synthesis}

A mixture containing $0.450 \mathrm{~g}$ of phosphorus pentabromide and $20 \mathrm{~mL}$ of solution 1 was stirred to form a red to brown solution. To the brown solution was added dropwise $4 \mathrm{~mL}$ of $2 \mathrm{M}$ lithium borohydride in tetrahydrofuran. The brown colour disappeared and bright orange liquid droplets in a colourless solution were observed. The appearance of the resulting mixture remained unchanged after being stirred for 24 hours. After being allowed to settle, a phase separation occurred to give a clear colourless supernatant and a yellow to orange liquid. The orange liquid was syringed out and kept under nitrogen.

\section{Purification}

A small amount of the orange liquid was diluted with ethanol to isolate a yellow powder which could be dispersed into ethanol by sonication. A drop of the solution was dried on a TEM grid in air for TEM characterization. 


\section{Synthesis of InP nanowires (Experiment 2)}

\section{Solution 1}

The preparation was the same as described for solution 1 in experiment 1.

\section{Synthesis}

A mixture containing $0.175 \mathrm{~g}$ indium trichloride, $0.340 \mathrm{~g}$ of phosphorus pentabromide and $5 \mathrm{~mL}$ of solution 1 was stirred for 36 hours. Within the first two hours after mixing, a brownish orange solution and some white solid were observed. The final product was an apple green solid which precipitated from a slightly green solution.

\section{Synthesis of Indium Phosphide Nanowires (Experiment 3)}

\section{Solution 1}

The preparation was the same as described for solution 1 in experiment 1 .

\section{Precursor Mixture}

$0.367 \mathrm{~g}$ indium trichloride and $0.7962 \mathrm{~g}$ of phosphorus pentabromide were added to 20 $\mathrm{mL}$ of solution 1 . The resulting mixture was stirred overnight to form a yellow solution with a few grains of green to brown solid precipitates. To the yellow solution was added dropwise $7 \mathrm{~mL}$ of $2 \mathrm{M}$ lithium borohydride in tetrahydrofuran. The resulting mixture was stirred for 8 hours to form a mixture of a bright yellow solid and a yellow to orange solution. The mixture was pumped under vacuum to remove tetrahydrofuran and toluene overnight.

\section{Injection and Growth}

$2 \mathrm{~mL}$ of the precursor mixture was added slowly to $10 \mathrm{~mL}$ of trioctylphosphine at $242{ }^{\circ} \mathrm{C}$ in 2 minutes. A $25{ }^{\circ} \mathrm{C}$ temperature drop was observed 8 minute after the injection. The resulting solution mixture was heated in between $250-260{ }^{\circ} \mathrm{C}$ for 1.5 hours. The solution was cooled in a water bath to room temperature.

\section{Purification}

After being cooled to room temperature, the reacted mixture was diluted with $15 \mathrm{~mL}$ of octane. The octane solution was centrifuged at $4000 \mathrm{rpm}$ for 10 minutes to isolate a fine brown solid precipitate. The precipitate was suspended in $20 \mathrm{~mL}$ of chloroform and 
$10 \mathrm{~mL}$ of water was added. The mixture was shaken vigorously before being centrifuged at $4000 \mathrm{rpm}$ for 3 minutes. A solid was found caught in between the aqueous fraction (top) and the chloroform fraction (bottom). The chloroform fraction was syringed out and the aqueous fraction discarded to isolate the solid. The solid was re-dispersed in $50 \mathrm{~mL}$ of water and the dispersion was centrifuged at $4000 \mathrm{rpm}$ for 5 minutes to isolate a solid precipitate. The dispersion-centrifugation process was repeated with $20 \mathrm{~mL}$ of ethanol. The final precipitate was suspended in $10 \mathrm{~mL}$ of chloroform.

\section{Synthesis of InP nanowires (Experiment 4)}

\section{Solution 1}

The preparation was the same as described for solution 1 in experiment 1 .

\section{Solution 2}

A mixture containing $0.450 \mathrm{~g}$ of phosphorus pentabromide and $20 \mathrm{~mL}$ of solution 1 was stirred to form a red to brown solution. To the brown solution was added dropwise $4 \mathrm{~mL}$ of $2 \mathrm{M}$ lithium borohydride in tetrahydrofuran. The brown colour disappeared and bright orange liquid droplets in a colourless solution were observed. The appearance of the resulting mixture remained unchanged after being stirred for 24 hours. After being allowed to settle, a phase separation occurred to give a clear colourless supernatant and a yellow to orange liquid. The orange liquid was syringed out and kept under nitrogen.

\section{Solution 3}

A mixture containing $0.064 \mathrm{~g}$ of indium trichloride and $10 \mathrm{~mL}$ trioctylphosphine was heated to $\sim 100{ }^{\circ} \mathrm{C}$ to form a clear colourless solution.

\section{Injection and Growth}

A mixture containing $1 \mathrm{~mL}$ of solution 2 and $2 \mathrm{~mL} \mathrm{~N}$-N-diethylaniline was injected into solution 3 at $250{ }^{\circ} \mathrm{C}$. A $\sim 100{ }^{\circ} \mathrm{C}$ temperature drop was observed due to vaporization of liquid tetrahydrofuran and toluene originated from solution 2. The temperature of the solution increased within an hour to $\sim 250{ }^{\circ} \mathrm{C}$. The mixture was heated in between 250 $280{ }^{\circ} \mathrm{C}$ for overnight. A dark brown solution was obtained. 


\section{Purification}

After cooled to room temperature, the reacted mixture was diluted with $15 \mathrm{~mL}$ of toluene. The toluene solution was centrifuged at $4000 \mathrm{rpm}$ for 3 minutes to isolate a brown solid precipitate. The precipitate was suspended in $25 \mathrm{~mL}$ of toluene and centrifuged at $4000 \mathrm{rpm}$ for 3 minutes. The suspension-centrifugation process was repeated 7 times with toluene and 2 times with methanol. The final dark precipitate was dissolved in $10 \mathrm{~mL}$ of toluene.

\section{Synthesis of InP nanowires (Experiment 5)}

\section{Solution 1}

The preparation was the same as described for solution 1 in experiment 1 .

\section{Solution 2}

A solution containing $0.200 \mathrm{~g}$ indium trichloride, $0.373 \mathrm{~g}$ of phosphorus pentabromide and $10 \mathrm{~mL}$ of solution 1 was stirred for 24 hours to form a greenish brown mixture. To the mixture was added dropwise $3.6 \mathrm{~mL}$ of $2 \mathrm{M}$ lithium borohydride in tetrahydrofuran. A light yellow solution formed consequently. After being stirred for 2 hours, the solution was pumped under vacuum for 12 hours to remove tetrahydrofuran and toluene. A grey to green liquid formed.

\section{Injection and Growth}

A mixture containing $0.5 \mathrm{~mL}$ of solution 2 and $1 \mathrm{~mL}$ of $\mathrm{N}, \mathrm{N}$-diethyaniline was injected into $10 \mathrm{~mL}$ of trioctylphosphine at $250{ }^{\circ} \mathrm{C}$ in two equal portions. A $12{ }^{\circ} \mathrm{C}$ temperature drop observed 5 minutes after the injection. The resulting mixture was heated in between $250-280{ }^{\circ} \mathrm{C}$ for 2 hours. The solution was cooled quickly by taking the reaction flask out from the heating mantle and allowed to rest on an aluminium mantle. 


\section{Purification}

After cooled to room temperature, the reacted mixture was diluted with $20 \mathrm{~mL}$ of toluene. The toluene solution was centrifuged at $4000 \mathrm{rpm}$ for 3 minutes to isolate a dark brown solid precipitate. The precipitate was suspended in $25 \mathrm{~mL}$ of toluene and centrifuged at $4000 \mathrm{rpm}$ for 3 minutes. The process was repeated 4 times with toluene and 3 times with methanol. The final dark precipitate was dissolved in $10 \mathrm{~mL}$ of chloroform.

\section{Synthesis of Indium Phosphide Nanowires (Experiment 6)}

\section{Solution 1}

The preparation was the same as described for solution 1 in experiment 1.

\section{Solution 2}

A mixture containing $0.226 \mathrm{~g}$ of phosphorous pentabromide and $5 \mathrm{~mL}$ of solution 1 was stirred to form a reddish brown solution. To the solution was added $1 \mathrm{~mL}$ of $2 \mathrm{M}$ lithium borohydride in tetrahydrofuran dropwise in 3 minutes. After all the gaseous products have escaped the system, an orangish yellow precipitate was isolated after allowed to settle.

\section{Solution 4}

A mixture containing $0.111 \mathrm{~g}$ of indium trichloride and $10 \mathrm{~mL}$ of $\mathrm{N}, \mathrm{N}$-diethylaniline was sonicated overnight to yield a deep brown solution with undissolved fine solid in it. It was shaken to distribute the solid before use.

\section{Solution 5}

Indium metal seeds were prepared in isobutylamine as detailed in experiment 3 in chapter 5.

\section{Reaction and Growth}

To $3.4 \mathrm{~mL}$ of $\mathrm{N}$,N-diethylaniline was added $1 \mathrm{~mL}$ of trioctylphosphine, $1.6 \mathrm{~mL}$ of solution 4 and $0.05 \mathrm{~mL}$ of 5 . The mixture was stirred for 10 minute and to it $1 \mathrm{~mL}$ of solution 2 was added. The colour of the solution changed from reddish brown to bright yellow and gaseous products observed. The mixture was then heated in an oil bath pre- 
heated to $\sim 205{ }^{\circ} \mathrm{C}$. A $22{ }^{\circ} \mathrm{C}$ temperature drop (of the oil bath) was observed. The colour of reacting mixture turned form bright yellow to orange and finally light brown within 5 minutes. The temperature of the oil bath increased to a stable temperature of $\sim 198{ }^{\circ} \mathrm{C}$. After heated at $198{ }^{\circ} \mathrm{C}$ for 2 hours, a deep brown solution formed. The flask containing the solution was taken out from the heating mantle and allowed to cool in air resting on an aluminium heating mantle. A deep brown precipitate and a transparent yellow supernatant were observed after allowed to settle.

\section{Purification}

After cooled to room temperature, the supernatant was discarded and the precipitate was suspended in $25 \mathrm{~mL}$ of toluene. The toluene solution was centrifuged at $1000 \mathrm{rpm}$ for 1 minute to isolate a solid precipitate. The purification process was repeated 3 times with toluene. The precipitate isolated was then suspended in methanol and centrifuged at $3000 \mathrm{rpm}$ for 1 minute to isolate a deep brown solid. The process was repeated three times. The final precipitate isolated was suspended in $10 \mathrm{~mL}$ of chloroform.

\section{Synthesis of Indium Phosphide Nanowires (Experiment 7)}

\section{Solution 1}

The preparation was the same as described for solution 1 in experiment 1 .

\section{Solution 2}

A mixture containing $1.213 \mathrm{~g}$ of phosphorus pentabromide and $10 \mathrm{~mL}$ of solution 1 was stirred to form a reddish brown solution. The solution was saturated with phosphorus pentabromide and residue phosphorus pentabromide crystals were observed.

\section{Solution 3}

A solution containing $5 \mathrm{~mL}$ of $2 \mathrm{M}$ lithium borohydride in tetrahydrofuran and $5 \mathrm{~mL}$ of trioctylphosphine was stirred to form a clear colourless solution. The solution was pumped under vacuum for 1 day to remove tetrahydrofuran. The resulting cloudy white suspension was sonicated under nitrogen over night to form a fine white cloudy suspension. 


\section{Solution 4}

A mixture containing $0.110 \mathrm{~g}$ of indium trichloride and $10 \mathrm{~mL}$ of trioctylphosphine was heated to $\sim 100{ }^{\circ} \mathrm{C}$ to form a clear colourless solution.

\section{Solution 5}

Indium metal seeds were prepared in isobutylamine as detailed in experiment 3 in chapter 5.

\section{Injection and Growth}

A mixture was prepared by combining $5 \mathrm{~mL}$ of trioctylphosphine, $0.1 \mathrm{~mL}$ of solution 4 , $0.3 \mathrm{~mL}$ of solution $2,0.1 \mathrm{~mL}$ of solution 3 and $0.05 \mathrm{~mL}$ of solution 5 . The mixture was quickly heated in an oil bath at $200{ }^{\circ} \mathrm{C}$. A bright orange mixture formed upon heating. The mixture was heated for 2 hours before allowed to cool in air. A yellow to orange solution was obtained.

\section{Purification}

After cooled to room temperature, the reacted mixture was diluted with $20 \mathrm{~mL}$ of hexane. The hexane solution was centrifuged at $4000 \mathrm{rpm}$ for 5 minutes to isolate a yellow to orange solid precipitate. The precipitate was suspended in $25 \mathrm{~mL}$ of hexane and centrifuged at $4000 \mathrm{rpm}$ for 5 minutes. The process was repeated 1 time with hexane and 3 times with methanol. The final solid precipitate was dissolved in $8 \mathrm{~mL}$ of chloroform.

\section{Synthesis of Indium Phosphide Nanowires (Experiment 8)}

\section{Solution 1}

The preparation was the same as described for solution 1 in experiment 1 .

\section{Solution 2}

The preparation was the same as described for solution 2 in experiment 7 .

\section{Solution 3}

A mixture containing $1.2 \mathrm{~mL}$ of $2 \mathrm{M}$ lithium borohydride in tetrahydrofuran and $10 \mathrm{~mL}$ of trioctylphosphine was stirred to form a clear colourless solution. The solution was 
pumped under vacuum to remove tetrahydrofuran. The resulting cloudy white suspension was heated to $250{ }^{\circ} \mathrm{C}$ under nitrogen.

\section{Solution 4}

Indium metal seeds were prepared in isobutylamine as detailed in experiment 3 in chapter 5. Isobutylamine was replaced by equalled volume of trioctylphosphine.

\section{Solution 5}

A mixture containing $0.1330 \mathrm{~g}$ of indium trichloride and $5 \mathrm{~mL}$ of trioctylphosphine was heated in an oil bath at $120{ }^{\circ} \mathrm{C}$ to form a clear colourless solution.

\section{Solution 6}

A mixture was prepared by mixing all of solution 5 with $5 \mathrm{~mL}$ of solution 2 .

\section{Injection and Growth}

A mixture containing all of solution 3 and $1 \mathrm{~mL}$ of solution 4 was stirred for 15 minutes. To the mixture was added slowly all of solution 6 via a dropping funnel over 1.5 hours and at $250{ }^{\circ} \mathrm{C}$. The reacting mixture was heated for an overall of 3 hours.

\section{Purification}

The solution was purified by washing with copious of toluene and a solid was isolated after centrifugation.

\section{Synthesis of Indium Phosphide Nanowires (Experiment 9)}

\section{Solution 1}

The preparation was the same as described for solution 1 in experiment 1 .

\section{Solution 2}

The preparation was the same as described for solution 2 in experiment 7.

\section{Solution 3}

The preparation was the same as described for solution 3 in experiment 8 . 


\section{Solution 4.}

A mixture containing $0.106 \mathrm{~g}$ of indium trichloride and $10 \mathrm{~mL}$ of trioctylphosphine was heated to $\sim 100{ }^{\circ} \mathrm{C}$ to form a clear colourless solution.

\section{Injection and Growth}

$0.2 \mathrm{~mL}$ of solution 3 was added to solution 4 to form a light grey solution at $250{ }^{\circ} \mathrm{C} .5$ minutes later, $0.6 \mathrm{~mL}$ of solution 3 and $1 \mathrm{~mL}$ of solution 2 was added dropwise (separately) into the grayish solution in an alternative fashion. The alteration time was 10 seconds and an overall of 54 minutes was required to complete the additions. The resulting solution was heated for a further 2 hours at $250{ }^{\circ} \mathrm{C}$. The solution was allowed to cool quickly in air by taking the reaction flask out from the heating mantle and allowed to rest on an aluminium mantle.

\section{Purification}

After cooled to room temperature, the reacted mixture was diluted with $20 \mathrm{~mL}$ of chloroform. The chloroform solution was centrifuged at $4000 \mathrm{rpm}$ for $5 \mathrm{mins}$ to isolate a brown solid precipitate. The precipitate was suspended in $25 \mathrm{~mL}$ of toluene and centrifuged at $4000 \mathrm{rpm}$ for 3 minutes. The process was repeated 3 times with toluene and 3 times with methanol. The final black solid precipitate was suspended in a solvent mixture consisted of $9 \mathrm{~mL}$ chloroform and $1 \mathrm{~mL}$ of butanol.

\section{Synthesis of Indium Phosphide Nanowires (Experiment 10)}

\section{Solution 1}

The preparation was the same as described for solution 1 in experiment 1 .

\section{Solution 2}

The preparation was the same as described for solution 2 in experiment 7.

\section{Solution 3}

The preparation was the same as described for solution 3 in experiment 8. 


\section{Solution 4}

A mixture containing $0.110 \mathrm{~g}$ of indium trichloride and $10 \mathrm{~mL}$ of trioctylphosphine was heated to $\sim 100{ }^{\circ} \mathrm{C}$ to form a clear colourless solution.

\section{Solution 5}

Indium metal seeds suspended in N,N-diethylaniline were prepared as detailed in experiment 4 in chapter 5 .

\section{Injection and Growth}

To $4.8 \mathrm{~mL}$ of solution 4 was added $0.25 \mathrm{~mL}$ of solution 5 at $250{ }^{\circ} \mathrm{C}$. To the resulting solution was added separately $0.5 \mathrm{~mL}$ of solution 3 and $1.2 \mathrm{~mL}$ of solution 2 dropwise (separately) in an alternative fashion. The alteration time was $\sim 10$ seconds and solution 3 was first to be consumed. The remaining solution 2 was added dropwise every 10 sec. An overall of 38 minutes was required to complete the additions. The resulting solution was heated for a further 2 hours in between $250-275{ }^{\circ} \mathrm{C}$. The solution was allowed to cool quickly in air by taking the reaction flaks out from the heating mantle and allowed to rest on an aluminium mantle.

\section{Purification}

After cooled to room temperature, the reacted mixture was diluted with $20 \mathrm{~mL}$ of chloroform. The chloroform solution was centrifuged at $4000 \mathrm{rpm}$ for 5 mins to isolate a brown solid precipitate. The precipitate was suspended in $25 \mathrm{~mL}$ of toluene and centrifuged at $4000 \mathrm{rpm}$ for 3 minutes. The process was repeated 3 times with toluene and 3 times with methanol. The final black solid precipitate was suspended in a solvent mixture of $9 \mathrm{~mL}$ chloroform and $1 \mathrm{~mL}$ butanol. 


\section{Synthesis of Indium Phosphide Nanowires (Experiment 11)}

\section{Solution 1}

A mixture containing $20 \mathrm{~mL}$ of $\mathrm{N}, \mathrm{N}$-diethylaniline and $5 \mathrm{~mL}$ of trioctylphosphine was heated and pumped under vacuum to degas for an overall one hour. A transparent reddish brown solution obtained and the flask was filled with nitrogen gas.

\section{Solution 2}

$5 \mathrm{~mL}$ of solution 1 at $79{ }^{\circ} \mathrm{C}$ was syringed and added to $0.519 \mathrm{~g}$ of phosphorus pentabromide. An orange solution formed. As it cooled a gel formed and the solution became turbid. To the solid gel at room temperature was added slowly $2.4 \mathrm{~mL}$ of $2 \mathrm{M}$ lithium borohydride in tetrahydrofuran in 2 minutes. Bubbles and gaseous products formed immediately. As the reaction proceeded, a brown liquid solution was formed from the gel. The solution was pumped under vacuum to remove tetrahydrofuran. The flask containing the solution was filled with nitrogen for storage.

\section{Solution 3}

Bismuth nanoparticles were prepared as detailed in experiment 5 in chapter 5 . To solution 2 were added $3 \mathrm{~mL}$ of the bismuth nanoparticles solution and $3 \mathrm{~mL}$ of trioctylphosphine. The mixture was pumped under vacuum to remove isobutylamine for 20 minutes. The flask was then filled with nitrogen gas.

\section{Solution 4}

Under nitrogen a colourless solution of $0.104 \mathrm{~g}$ of indium trichloride in $7 \mathrm{~mL}$ of trioctylphosphine mixture was heated to $250{ }^{\circ} \mathrm{C}$.

\section{Injection and Growth}

To solution 4 was injected $0.5 \mathrm{~mL}$ of solution 3 six times with a time interval of 3 minutes. For each injection the injection temperature was recorded to be in between $250-257{ }^{\circ} \mathrm{C}$. The mixture was then allowed to be heated $\sim 250-280{ }^{\circ} \mathrm{C}$ for 3 hours and 14 minutes. The black solution mixture was then taken out from the heating mantle and allowed cool in air resting on an aluminium mantle. 


\section{Purification}

After cooled to room temperature and allowed to settle overnight, a black solid precipitate crashed out from the solution. The supernatant was cloudy white. The supernatant was discarded and the black solid suspended in $15 \mathrm{~mL}$ chloroform. The resulting solution was centrifuged at $4000 \mathrm{rpm}$ for 5 minutes to isolate a black solid precipitate. The purification process was repeated four times with chloroform and twice with methanol. The final precipitate isolated was suspended in $10 \mathrm{~mL}$ of toluene. The solution was used for TEM studies.

\section{Acid treatment}

The final precipitate was stirred with $25 \mathrm{~mL}$ of $0.1 \mathrm{M}$ hydrochloric acid in methanol at room temperature for 3 hours to remove residual bismuth nanoparticles to afford nanowires sufficiently clean for TEM study. 


\subsection{References}

1. Wagner, R. S.; Ellis, W. C. Apply. Phys. Lett. 1964, 4, 89-90.

2. Wu, Y.; Yang, P. J. Am. Chem. Soc. 2001, 123, 3165-3166.

3. Novotny, C. J.; Yu, P. K. L. Appl. Phys. Lett. 2005, 87, 203111/1-203111/5.

4. Xiong, Q. H.; Wang, J.; Eklund, P. C. Nano Lett. 2006, 6, 2736-2742.

5. Johansson, J.; Karlsson, L. S.; Svensson, C. P. T.; Mårtensson, T.; Wacaser, B.; Deppert, K.; Samuelson, L.; Seifert, W. Nature Mater. 2006, 5, 574-580.

6. Bhunia, S.; Kawamura, T.; Fujikawa, S.; Nakashima, H.; Furukawa, K.; Torimitsu, K.; Watanabe, Y. Thin Solid Films 2004, 464-465, 244-247.

7. Bhunia, S.; Kawamura, T.; Watanabe, Y.; Fujikawa, S.; Tokushima, K. Appl. Phys. Lett. 2003, 83, 3371-3373.

8. Mattila, M.; Hakkarainen, T.; Lipsanen, H.; Jiang, H.; Kauppinen, E. I. Appl. Phys. Lett. 2007, 90, 033101/1-033101/3.

9. Mattila, M.; Hakkarainen, T.; Mulot, M.; Lipsanen, H. Nanotechnology 2006, 17, 1580-1583.

10. Duan, X. F.; Huang, Y.; Cui, Y.; Wang, J. F.; Lieber, C. M. Nature 2001, 409, 66-69.

11. Wang, J. F.; Gudiksen, M. S.; Duan, X. F.; Cui, Y.; Lieber, C. M. Science 2001, $293,1455-1457$.

12. Duan, X. F.; Lieber, C. M. Adv. Mater. 2000, 12, 295-302. 
13. Gudiksen, M. S.; Wang, J. F.; Lieber, C. M. J. Phys. Chem. C 2001, 105, 4062-4064.

14. Trentler, T. J.; Hickman, K. M.; Goel, S. C.; Viano, A. M.; Gibbons, P. C.; Buhro, W.E. Science 1995, 270, 1791-1794.

15. (a) Trentler, T. J.; Goel, S. C.; Hickman, K. M.; Viano, A.M.; Chiang, M. Y.; Beatty, A. M.; Gibbons, P. C.; Buhro, W. E. J. Am. Chem. Soc. 1997, 119, 2172-2181. (b) Wang, F. D.; Dong, A. G.; Sun, J. W.; Tang, R.; Yu, H.; Buhro, W. E. Inorg. Chem. 2006, 45, 7511-7521. (c) Wang, F. D.; Yu, H.; Li, J. B.; Hang, Q. L.; Zemlyanov, D.; Gibbons, P. C.; Wang, L. W.; Janes, D. B.; Buhro, W. E. J. Am. Chem. Soc. 2007, 129, 14327-14335.

16. (a) Micic, O. I.; Curtis, C. J.; Jones, K. M.; Sprague, J. R.; Nozik, A. J. J. Phys. Chem. 1994, 98, 4966-4969. (b) Micic, O. I.; Sprague, J. R.; Curtis, C. J.; Jones, K. M.; Machol, J. L.; Nozik, A. J.; Giessen, H.; Fluegel, B.; Mohs, G.; Peyghambarian, N. J. Phys. Chem. 1995, 99, 7754-7759. (c) Lucey, D. W.; MacRae, D. J.; Furis, M.; Sahoo, Y.; Cartwright, A. N.; Prasad, P. N. Chem. Mater. 2005, 17, 3754-3762.

17. Wilberg, E.; Muller-Schiedmayer, G.; Chemische Berichte 1959, 92, 2372-2384.

18. Greenwood, N. N.; Earnshaw, A. Chemistry of The Elements, ButterworthHeinemann LTD, Oxford, United Kingdom, 1994.

19. Yu, H.; Li, J.; Loomis, R.; Wang, L. W.; Buhro, W. E. Natur. Mater. 2003, 2, 517-520.

20. Gudiksen, M. S.; Wang, J. F.; Lieber, C. M. J. Phys. Chem. B 2002, 106, 40364039.

21. Fanfair, D. D.; Korgel, B. A. Crystal Growth and Design 2005, 5, 1971-1976.

22. Davidson, F. M., III; Lee, D. C.; Fanfair, D. D.; Korgel, B. A. J. Phys. Chem. C 2007, 111, 2929-2935. 
23. Cahn, R. W. Adv. Phys. 1954, 3, 363-445.

24. (a) Hurle, D. T. J.; Rudolph, P. J. Cryst. Growth 2004, 264, 550-564. (b) Hurle, D. T. J. J. Cryst. Growth 1995, 147, 239-250.

25. Davidson, F. M.; Schricker, A. D.; Wiacek, R.; Korgel, B. Adv. Mater. 2004, 16, 646-649.

26. Davidson, F. M., III; Wiacek, R.; Korgel, B. A. Chem. Mater. 2005, 17, 230-233.

27. Li, Q.; Gong, X. G.; Wang, C. R.; Wang, J.; Ip; K.; Hark, S. Adv. Mater. 2004, 16, 1436-1440.

28. Joyce, H. J.; Gao, Q.; Tan; H. H.; Jagadish, C.; Kim, Y.; Zhang, X.; Guo, Y. Zou, J. Nano Letters 2007, 7, 921-926.

29.(a) Chen, H.; Gao, Y.; Zhang, H.; Liu, L.; Yu, H.; Tian, H.; Xie, S.; Li, J. J. Phys. Chem. B. 2004, 108, 12038-12043.

30. Guzelian, A. A.; Katari, J. E. B.; Kadavanich, A. V.; Banin, U.; Hamad, K.; Juban, E.; Alivisatos, A. P.; Wolters, R. H.; Arnold, C. C.; Heath, J. R. J. Phys. Chem. 1996, 100, 7212-7219.

31. Levinstein, M.; Rumyantsev, S.; Shur, M. Handbook Series on Semiconductor Parameter Vol. 1., World Scientific, London, United Kingdom, 1999.

32. Lilly, G. Daniel; Lee, Jaebeom; Sun, Kai; Tang, Zhiyong; Kim, Ki-Sub; Kotov, Nicholas A. J. Phys. Chem. C 2008, 112, 370-377. 


\section{Chapter 7 Solution Synthesis of Crystalline Zinc Phosphide Nanoparticles}

\section{Introduction}

This chapter deals with the establishment of a new method that could be used to prepare crystalline zinc phosphide $\left(\mathrm{Zn}_{3} \mathrm{P}_{2}\right)$ nanoparticles. The establishment based itself on previous work in which indium phosphide nanowires were prepared from indium trichloride, lithium borohydride and phosphorus pentabromide (please see chapter 6 for details). Zinc phosphide was expected to form from reactions of zinc chloride, lithium borohydride and phosphorus pentabromide. Owing to the research target was crystalline nanoparticles instead of nanowires, the dropwise addition procedure which was necessary in the synthesis of indium phosphide nanowires has been replaced by an immediate/multiple-injection procedure. Trioctylphosphine, N,N-diethylaniline and oleylamine have been tested as reaction solvents and the results of the tested reactions were described in sections 7.2-7.4. The three solvents were chosen for reasons discussed in section 7.6.

Much research work on zinc phosphide has been focused on the fabrication of zinc phosphide thin films. Methods traditionally employed in the synthesis of bulk zinc phosphide involve direct combination of elemental zinc and phosphorus in a hot furnace and are not readily adaptable to nanostructures preparation. ${ }^{1-6}$ It was not until recently that methods modified from the traditional have allowed the preparation of zinc phosphide nanostructures. Research work on the solution phase synthesis of zinc phosphide nanocrystals has been scarce and only three reports were found in the literature. ${ }^{7-9}$ The most recent solution phase synthesis of zinc phosphide was reported by Green and Paul O'Brien in 2001. ${ }^{7}$ They prepared zinc phosphide nanoparticles of 3-5 $\mathrm{nm}$ in size by reacting dimethylzinc $\left(\mathrm{Me}_{2} \mathrm{Zn}\right)$ and ditert-butylphosphine $\left(\mathrm{HP}\left({ }^{\mathrm{t}} \mathrm{Bu}\right)_{2}\right)$ in trioctylphosphie oxide or 4ethylpyridine. However, the nanoparticles were poorly crystalline and no XRD and SAED pattern were observed. Buhro et. al. produced zinc phosphide nanoparticles from a metalloorganic approach in $1994 .{ }^{8}$ The earliest detailed research in zinc phosphide 
nanoparticles finds itself in a report by Weller and coworkers in 1985, detailing the preparation of zinc phosphide nanoparticles of 1-2 nm from phosphine $\left(\mathrm{PH}_{3}\right)$ and zinc chloride in aqueous solution. ${ }^{9}$ However in both Weller's and Buhro's reports no TEM or XRD evidences were given to corroborate that the particles they prepared were of zinc phosphide $\left(\mathrm{Zn}_{3} \mathrm{P}_{2}\right)$.

All experiments reported in this chapter aimed at finding a new synthesis of zinc phosphide using zinc chloride, lithium borohydride and phosphorus pentabromide. It was hoped that highly crystalline zinc phosphide nanoparticles could be prepared and fully characterized by HRTEM and PXRD techniques. The experiments were summarized with brief details in table 7.0. This piece of research work was not conducted in a pairwise-comparison manner as each experiment has at least two reaction parameters that were different from others. New reaction parameters were assigned to each experiment in order to improve the synthesis from previous experiments. Experiments 2 and 3 involved the use of phosphorus pentabromide as phosphorus source as in the synthesis of indium phosphide nanowires described in chapter 6. Experiments 4-5 involved the use of hydrogen phosphide $(\mathrm{PH})_{\mathrm{x}}$ instead of phosphorus pentabromide directly. Hydrogen phosphide was prepared as an orange precipitate by reacting phosphorus pentabromide with lithium borohydride and was dissolved in N-N-diethylaniline for transfer. Unless otherwise mentioned, the words "lithium borohydride" refers to a suspension of lithium borohydride in trioctylphosphine free from tetrahydrofuran. 
Table 7.0 Table of experiments.

\begin{tabular}{|c|c|c|c|c|c|c|}
\hline \multirow[t]{2}{*}{ Experiment } & \multicolumn{2}{|c|}{$\begin{array}{c}\text { Temperature in } \\
{ }^{\circ} \mathrm{C}\end{array}$} & Solvent & Surfactant & $\begin{array}{c}\text { Time } \\
\text { in } \\
\text { hrs }\end{array}$ & Sequences \\
\hline & Injection & Growth & & & & \\
\hline 2 & 212 & $170-180$ & $\begin{array}{l}\text { NNDA, } \\
20 \mathrm{~mL}\end{array}$ & NNDA & 25 & $\begin{array}{l}\text { 1. } \mathrm{ZnCl}_{2} \text { and } \mathrm{Na} \text { were reacted in NNDA. } \\
\text { 2. }(\mathrm{PH})_{\mathrm{x}} \text { was injected to }(1) \text {. }\end{array}$ \\
\hline 3 & $340-310$ & 310 & $\begin{array}{l}\mathrm{TOP}^{* 1} \\
5 \mathrm{~mL}\end{array}$ & TOP & 3 & $\begin{array}{l}\text { 1. } \mathrm{ZnCl}_{2} \text { was dissolved in } \mathrm{TOP} \\
\text { 2. Multiple injections. } \mathrm{PBr}_{5} \text { and } \mathrm{LiBH}_{4} \\
\text { were simultaneously injected into (1) } \\
\text { every } 5 \text { minutes. }\end{array}$ \\
\hline 4 & 330 & $320-300$ & $\begin{array}{l}\text { TOP, } \\
9 \mathrm{~mL}\end{array}$ & TOP & 3 & $\begin{array}{l}\text { 1. } \mathrm{ZnCl}_{2} \text { was dissolved in TOP. } \\
\text { 2. } \mathrm{PBr}_{5} \text { and } \mathrm{LiBH}_{4} \text { were } \\
\text { simultaneously injected into (1). }\end{array}$ \\
\hline 5 & 207 & 212 & $\begin{array}{l}\mathrm{NNDA}^{* 2}, \\
16 \mathrm{~mL}\end{array}$ & NNDA & 20 & $\begin{array}{l}\text { 1. } \mathrm{ZnCl}_{2} \text { was dissolved in TOP. } \\
\text { 2. }(\mathrm{PH})_{\mathrm{x}} \text { and } \mathrm{LiBH}_{4} \text { were mixed. } \\
\text { 3. }(2) \text { was injected to }(1) \text {. }\end{array}$ \\
\hline 6 & 197 & 200 & $\begin{array}{l}\mathrm{OLA}^{* 3} \\
16 \mathrm{~mL}\end{array}$ & OLA & 16 & $\begin{array}{l}\text { 1. } \mathrm{LiBH}_{4} \text { was added to } \mathrm{ZnCl}_{2} \text { in TOP } \\
\text { 2. }(\mathrm{PH})_{\mathrm{x}} \text { was injected into }(1)\end{array}$ \\
\hline
\end{tabular}

${ }^{1}$ Trioctylphosphine; ${ }^{2}$ N,N-diethylaniline $*^{3}$ Oleyamine. 


\subsection{Reaction of Zinc Chloride and Lithium Borohydride in Oleylamine (Experiment 1)}

Zinc chloride was dissolved in oleylamine to form a clear and nearly colourless solution. The solution reacted with lithium borohydride in tetrahydrofuran at room temperature as evidenced by the release of gaseous products. The reacted mixture remained colourless at this point. After being heated to $\sim 100{ }^{\circ} \mathrm{C}$, the mixture turned into a light yellow solution. The colour change may be due to the formation of a very small amount of zinc metal nanoparticles. However no direct evidence of the formation of zinc nanoparticles was available. No characterization was conducted to confirm the presence of zinc metal nanoparticles. The colour change was not sufficiently significant to be held as an absolute indication of the formation of zinc metal nanoparticles.

\subsection{Reaction of Zinc Chloride and Sodium in N,N- Diethylaniline (Experiment 2)}

Zinc chloride dissolved in N,N-diethylaniline was treated with sodium metal and the solution mixture heated to $\sim 200{ }^{\circ} \mathrm{C}$. The colour of solution changed from orange to dark grey. Such colour change was sufficiently significant to be held as an indicative of the formation of zinc metal nanoparticles. The solution was treated with hydrogen phosphide $(\mathrm{PH})_{\mathrm{x}}$ at $200{ }^{\circ} \mathrm{C}$ in an attempt to synthesize zinc phosphide. A brown to black solid was isolated from the solution. From SAED, the solid contained zinc metal nanowires as the only crystalline species (figure 7.1). The observation proved that zinc metal was formed in the sodium reduction reaction. However, the actual size and form of zinc metal nanostructures present prior to the addition of hydrogen phosphide at $200{ }^{\circ} \mathrm{C}$ could not be directly confirmed because the nanowires observed could be the results of particles aggregation or particle growth. 

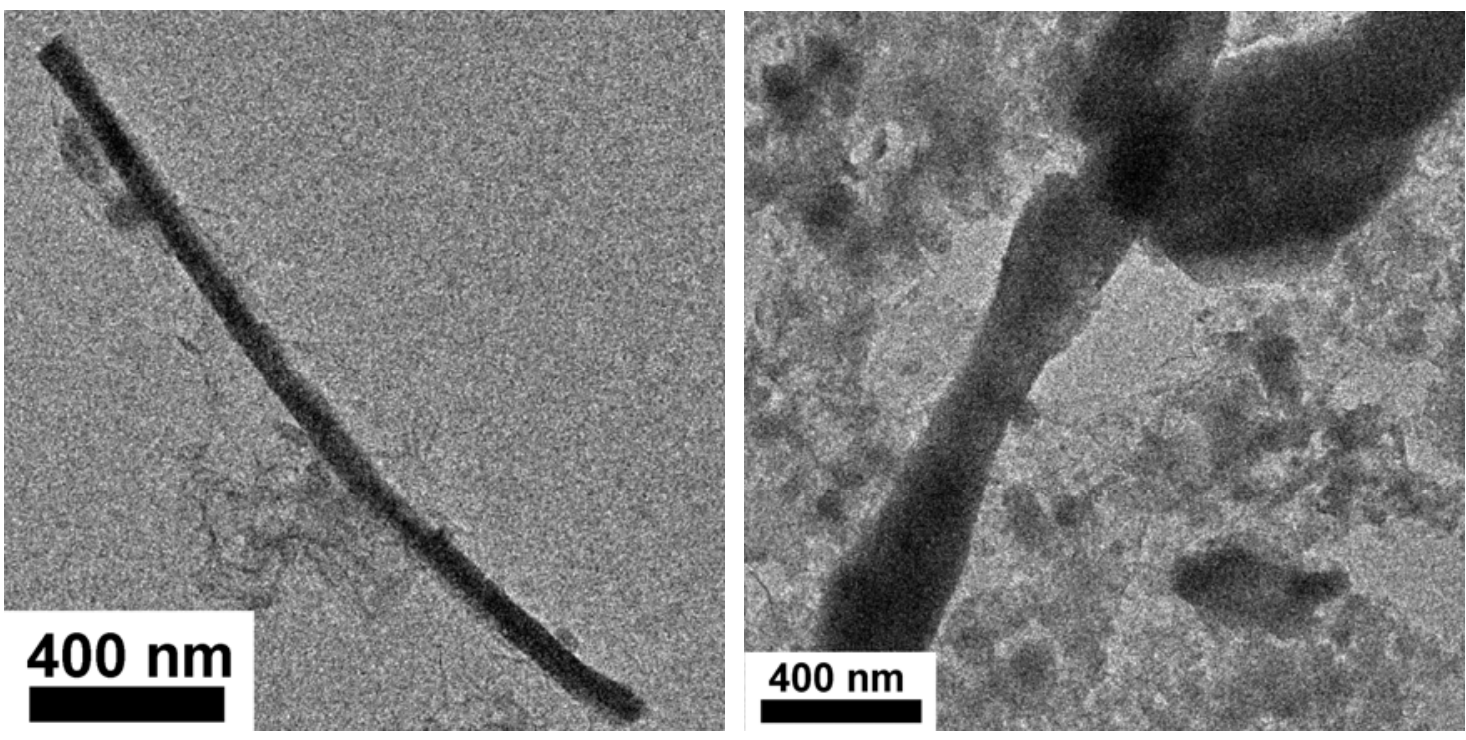

Figure 7.1 TEM images of zinc metal nanowires.

\subsection{Reactions of Zinc Chloride, Lithium Borohydride and Phosphorus Pentabromide in Trioctylphosphine (Experiment 3 and 4)}

Experiment 3 and 4 both involved the injections of phosphorus pentabromide and lithium borohydride into zinc chloride dissolved in trioctylphosphine. In experiment 3 the injections was carried out twice and in experiment 4 once. Both Experiments 3 and 4 were carried out at $\sim 330{ }^{\circ} \mathrm{C}$ aiming that a high reaction temperature would lead to better particle crystallinity. However, the reactions failed to produce any crystalline materials. From TEM both reactions produced large ensembles up to several hundred nanometers (figure 7.3 a and $b$ ). The ensembles were comprised of nanoparticles of $\sim 20 \mathrm{~nm}$ in size. From SAED, the nanoparticles were amorphous. EDS analysis (figure $7.3 \mathrm{c}$ and $\mathrm{d}$ ) showed that both ensembles contained zinc and phosphorus however both have zinc to phosphorus ratios other than 3:2 as the formula $\mathrm{Zn}_{3} \mathrm{P}_{2}$ would suggest. 

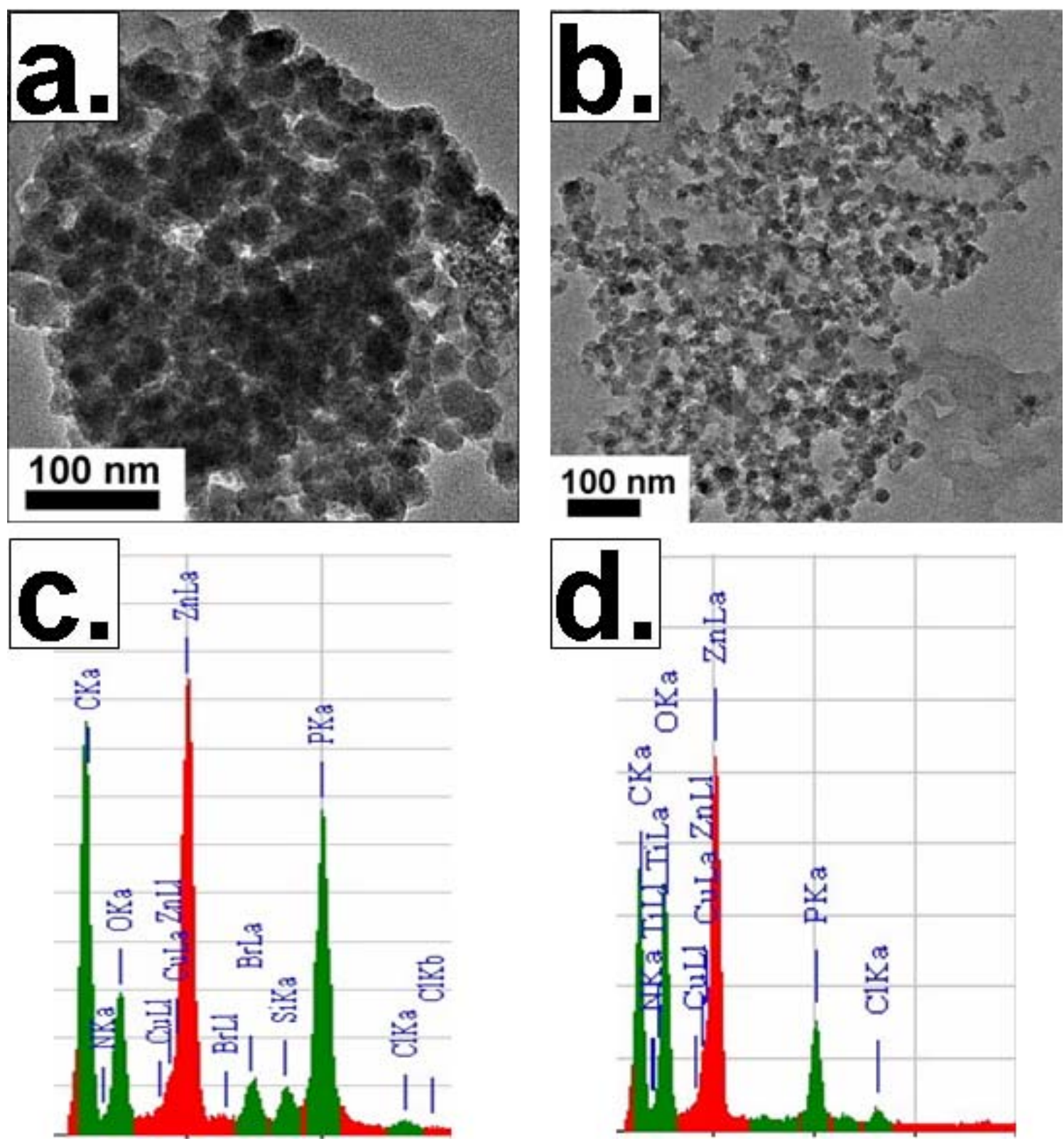

Figure 7.3 TEM images and EDS spectra. (a) A TEM image of nanoparticles obtained from experiment 3; (b) a TEM image of nanoparticles obtained from experiment 3; (c) and (d) EDS spectra for (a) and (b) respectively. 
Although the reactions failed to produce crystalline materials, however, nanoparticles containing both zinc and phosphorus were obtained. Trioctylphosphine was suspected to cause the formation of amorphous nanoparticles due to its strong coordinating strength. Therefore a reaction similar to experiment 4 was carried out in an amine based solvent to test if crystalline materials could be produced. The reason trioctylphosphine came under suspicion and the reason an amine solvent was chosen as a replacement were discussed in section 7.6.

\subsection{Reaction of Zinc Chloride, Lithium Borohydride and Phosphorus Pentabromide in N,N-diethylaniline (Experiment 5)}

Phosphorus pentabromide was first treated with lithium borohydride to produce hydrogen phosphide as an orange solid. Zinc chloride dissolved in N,N-diethylaniline was then treated with hydrogen phosphide and the mixture was heated and maintained at refluxing condition for 20 hours at $210{ }^{\circ} \mathrm{C}$. A brown solid product was isolated after purification. Xray diffraction analysis (figure 7.4 a) showed that the product contained a crystalline material. The diffraction pattern of the material matched that of zinc phosphide $\left(\alpha-\mathrm{Zn}_{3} \mathrm{P}_{2}\right)$ found in the literature. ${ }^{10}$

A TEM study revealed that the zinc phosphide $\left(\alpha-\mathrm{Zn}_{3} \mathrm{P}_{2}\right)$ synthesized were in the form of aggregated nanoparticles. As may be seen in figure $7.4 \mathrm{~b}$, the aggregates were of size up to several hundred nanometers. No specific shape was observed for the aggregates. The TEM study also revealed the presence of an amorphous species in the form of segregated nanoparticles. The nanoparticles have an average diameter of $\sim 10 \mathrm{~nm}$. The chemical identity of the nanoparticles was unsolved due to the absence of crystallinity and the presence of impurities.

This experiment proved that crystalline $\alpha-Z_{3} \mathrm{P}_{2}$ nanoparticles could be synthesized from zinc chloride, hydrogen phosphide and lithium borohydride at $\sim 200{ }^{\circ} \mathrm{C}$ in an amine based solvent. However, the crystalline nanoparticles aggregated into large ensembles. To prepare 
segregated crystalline nanoparticles of zinc phosphide a similar reaction was carried out in oleylamine. The reaction was detailed in the following section. The reason oleylamine was chosen was discussed in section 7.6.
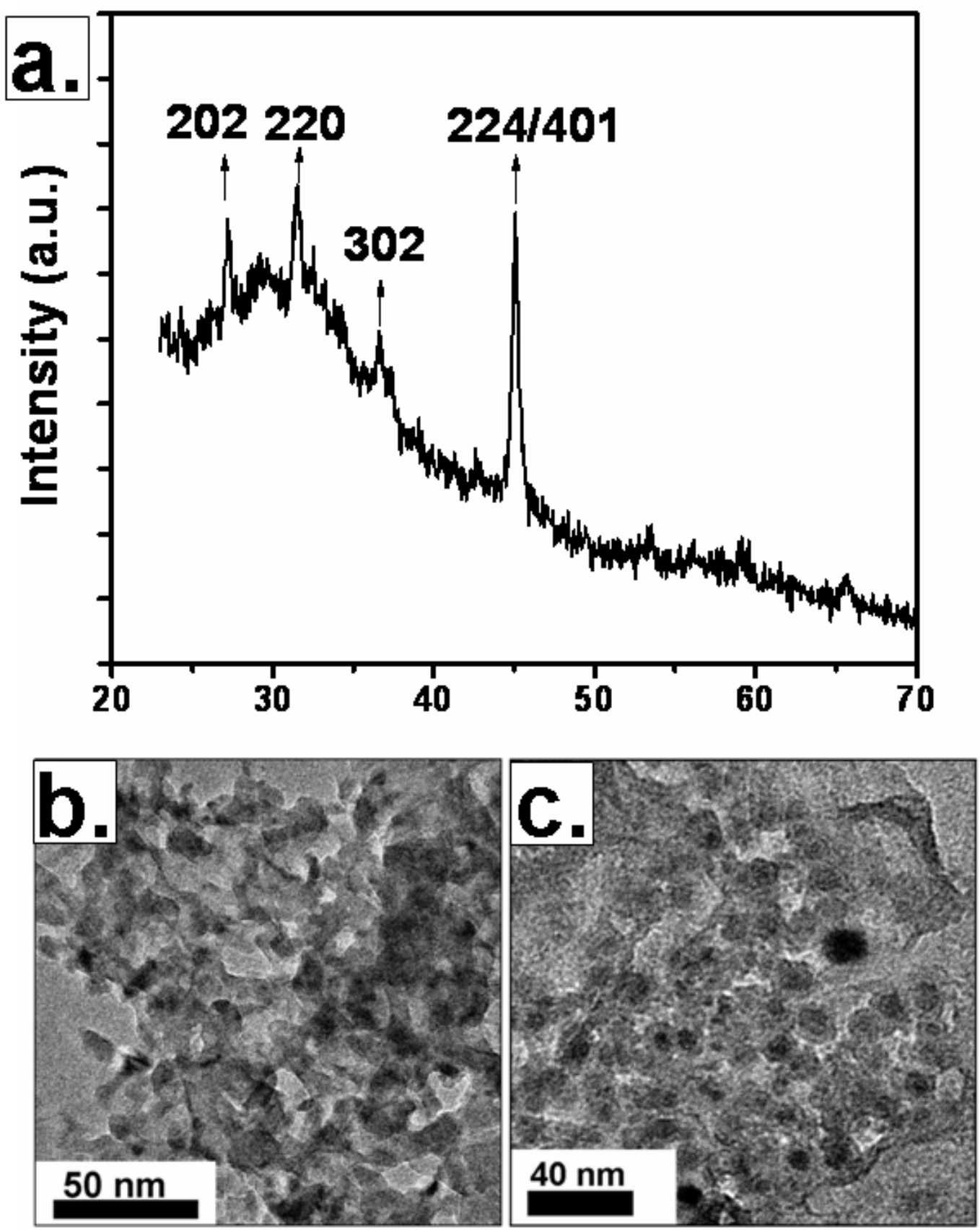

Figure 7.4 (a) a PXRD diffractogram of zinc phosphide $\left(\alpha-\mathrm{Zn}_{3} \mathrm{P}_{2}\right)$; (b) a TEM image of aggregated $\alpha-Z_{3} P_{2}$ nanocrystals and (c) a TEM image of amorphous spherical nanoparticles around 10-20 $\mathrm{nm}$. 


\subsection{Reactions of Zinc Chloride, Lithium Borohydride and Phosphorus Pentabromide in Oleylamine (Experiment 6)}

Hydrogen phosphide prepared from phosphorus pentabromide and lithium borohydride was injected to a solution of zinc chloride in oleylamine pre-heated to $200{ }^{\circ} \mathrm{C}$. The resulting mixture was heated for 16 hours. A deep brown solid product was isolated after purification. X-ray diffraction analyses revealed the presence of a crystalline material. The diffraction pattern of the material matched that of $\alpha-\mathrm{Zn}_{3} \mathrm{P}_{2} .{ }^{10}$ The peaks have been indexed as may be seen figure 7.50. The strongest peak observed before chloroform/butanol wash was due to impurities. Purer $\alpha-Z_{3} \mathrm{P}_{2}$ was obtained after extensive chloroform/butanol wash. No zinc oxide or metallic zinc was observed in the XRD diffractogram.

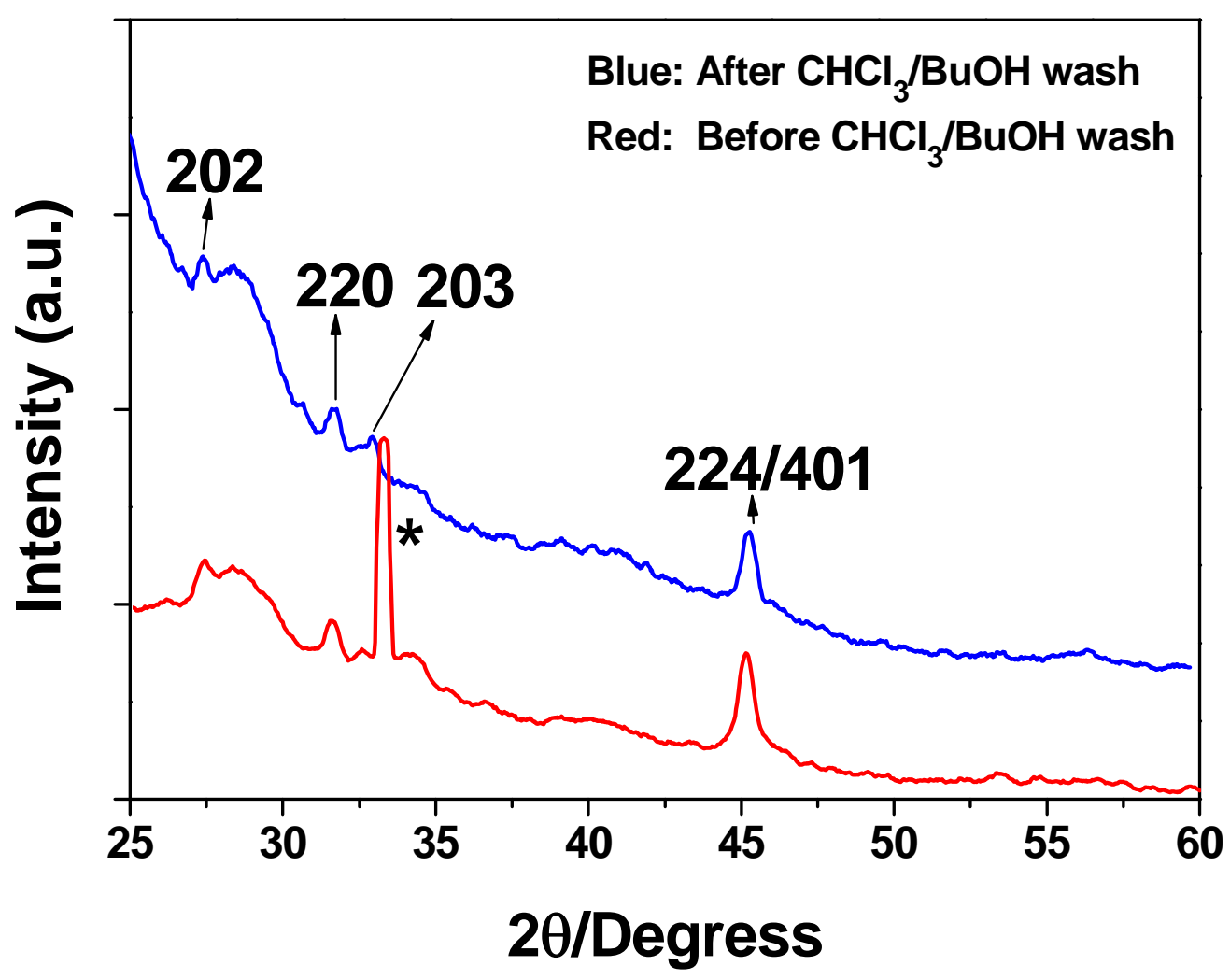

Figure 7.50 PXRD diffractograms of $\alpha-Z_{n} P_{2}$ prepared in oleylamine. Purer $\alpha-Z_{3} P_{2}$ was obtained after removal of impurities (as indicated $*$ ). 


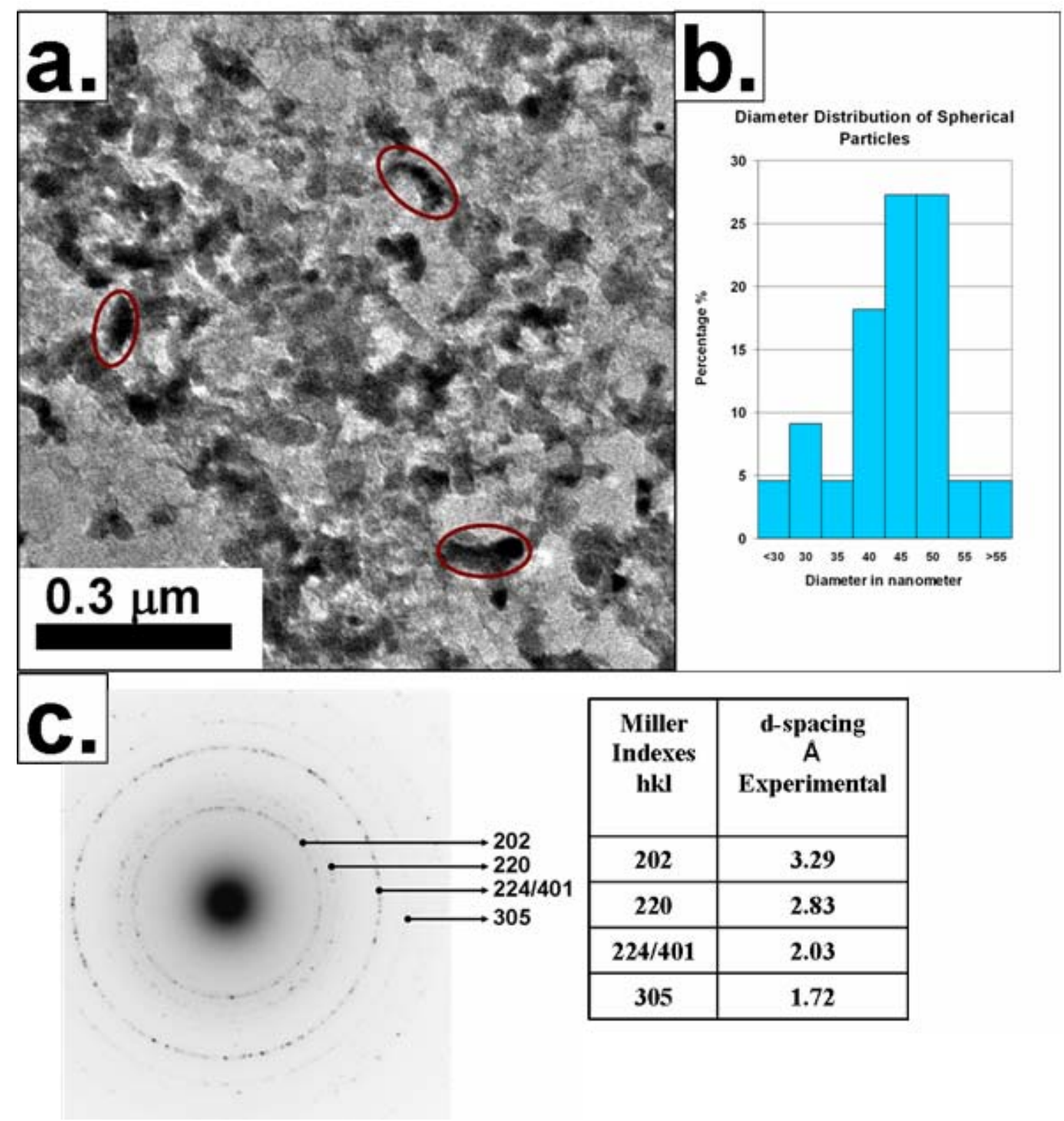

Figure 7.51 (a) a low magnification TEM image of nanorods and nanoparticles; (b) a size distribution histogram of spherical zinc phosphide nanoparticles. Red ovals highlight some of the rod-shape zinc phosphide nanoparticles; (c) A SAED pattern of $\alpha-Z_{3} \mathrm{P}_{2}$ nanoparticles. 
The impurities present in the PXRD pattern before chloroform/butanol wash cannot be fully identified due to the singularity of datum. Purer $\alpha-Z_{3} P_{2}$ was obtained after chloroform/butanol wash and the strongest peak at $2 \theta$ of $31.8^{\circ}$ disappeared. The disappearance of the strongest peak after wash suggested that the peak was not due to preferential orientation of the lattice plane (203) which should be weakly diffracted at $2 \theta$ value of $\sim 32^{\circ}$. It is also unlikely that a thermodynamically favoured crystal facet grown during the synthesis should exhibit a propensity to react with solvents such as chloroform and butanol.

Three different nanostructures were identified in digital TEM images. As may be seen in figure 7.51a, these include: (i) spherical nanoparticles, (ii) elongated rod-like nanoparticles (marked with dark red oval) and (iii) random net-like structures. Both (i) and (ii) exhibited dark contrast while (iii) exhibited relatively light contrast.

The spherical and rod-like nanoparticles were both crystalline and both contributed to a SAED diffraction pattern recorded (figure $7.51 \mathrm{c}$ ). The spherical nanoparticles were found to have an average diameter of $42.6 \pm 7.8 \mathrm{~nm}$. The elongated rod-like nanoparticles were found to have an average diameter of $33.3 \pm 3.5 \mathrm{~nm}$ and an average length of $72.2 \pm 10.7 \mathrm{~nm}$. The rods have an average aspect ratio of 2 . The net-like structures were found to be amorphous and to contain silicon and oxygen.

The spherical nanoparticles were the dominant species representing $72 \%$ of all crystalline nanostructures and the remaining 26\% were rod-like nanoparticles. The size distribution of the spherical nanoparticles was moderately monodispersed as may be seen in figure $7.51 \mathrm{~b}$. The rods have an average diameter $10 \mathrm{~nm}$ smaller than that of the spherical nanoparticles. The existence of two groups of nanoparticles of different size in a one-pot synthesis may be explained by the Ostwald ripening process in which large particles grow at the expenses of small nanoparticles. However, there is no obvious explanation to account for two different shapes. 
Figure 7.51c represents the SAED pattern derived from both the spherical nanoparticles and rod-like nanoparticles. The SAED pattern corresponded to only one crystalline material and therefore both the spherical and rod-like nanoparticles adopted the same crystal structure. The rings pattern indicated the polycrystalline nature of the particles. Each ring was indexed indicating that tetragonal $\alpha-Z_{3} \mathrm{P}_{2}$ was responsible for the SAED pattern. From the indexed SAED, lattice parameter a and c were calculated to be $8.06 \AA$ and $11.39 \AA$ using equation 5.2 derived in reference 11. The lattice parameter matched well those of $8.060 \AA$ and $11.400 \AA$ of a reference. ${ }^{10}$

$$
\frac{1}{d^{2}}=\frac{\left(h^{2}+k^{2}\right)}{a^{2}}+\frac{l^{2}}{c^{2}}----- \text { equation } 5.2
$$

HRTEM studies revealed nanoparticles of single crystalline nature. Figure 7.52 showed a rod-shape single crystalline nanoparticle with lattice fringes spanning across more than 20 $\mathrm{nm}$ (region marked by yellow line). Amorphous species (marked by the red line) distinguishable from the amorphous carbon film (region marked by lime line) were observed around the crystal. Single crystalline spherical nanoparticles were also observed as represented by figure 7.53 


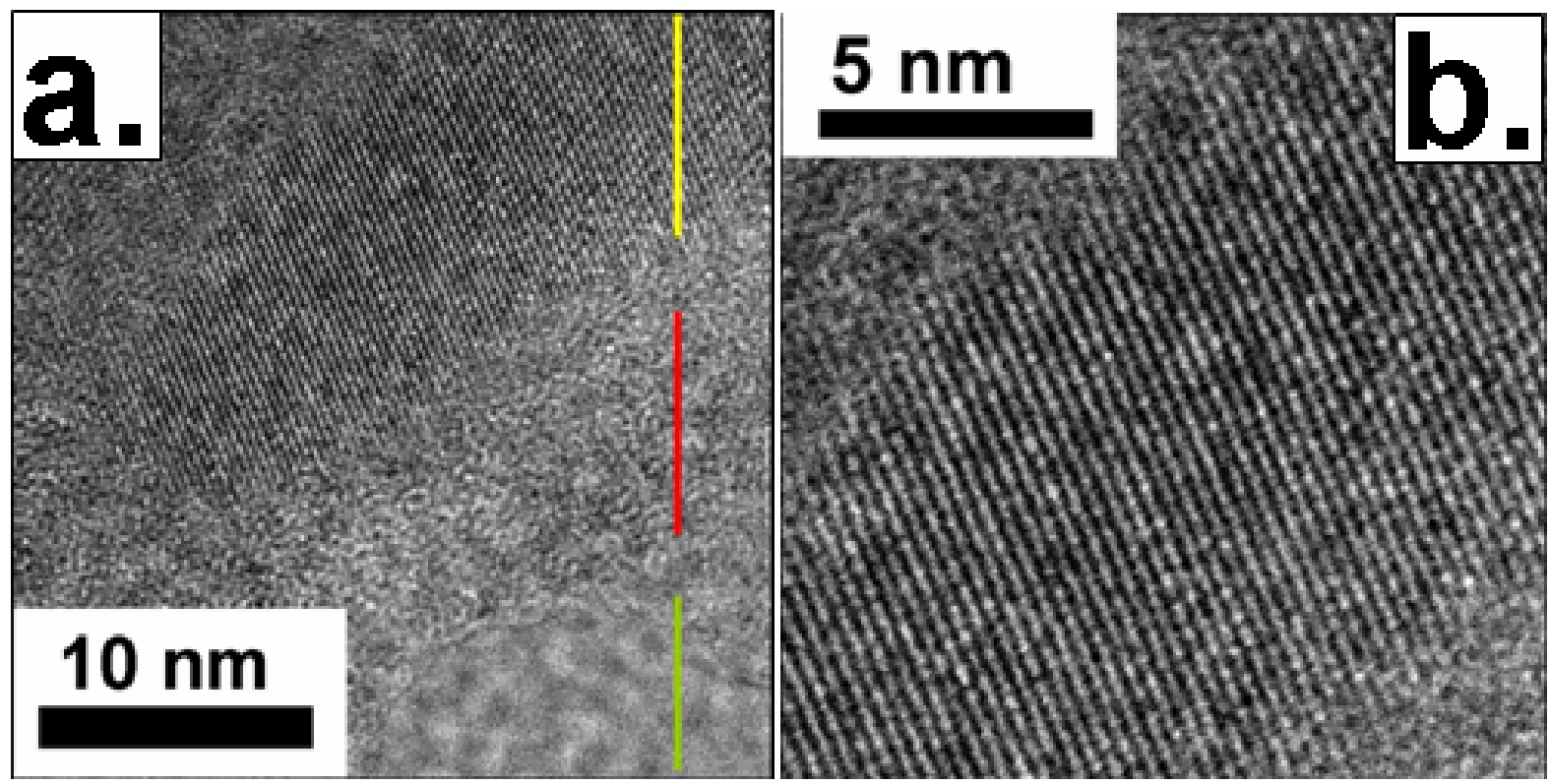

Figure 7.52 (a) a HRTEM image of a rod-like single crystal of zinc phosphide surrounded by a layer of amorphous material and (b) a closer look at the lattice fringes of the rod-like particle.

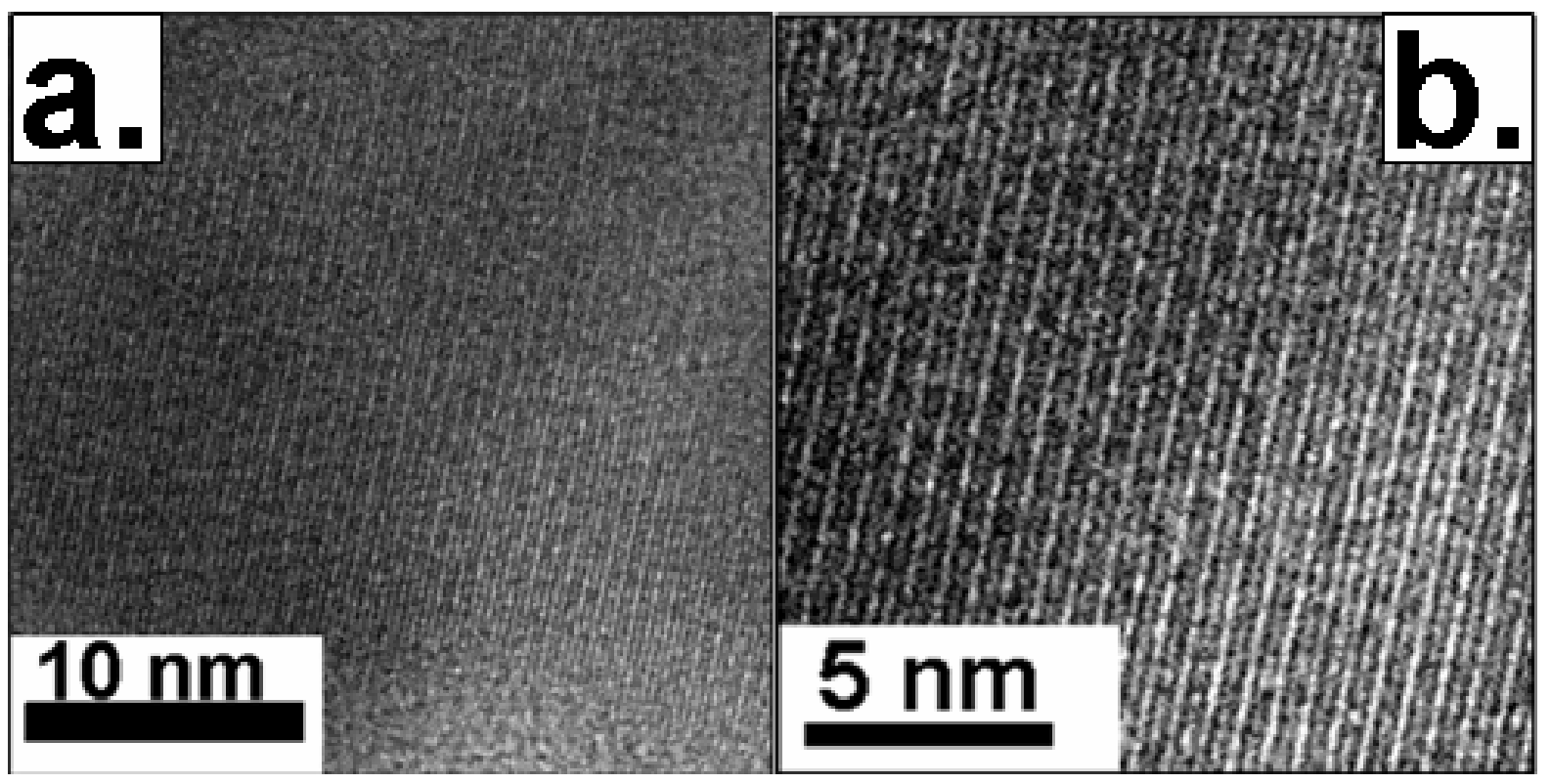

Figure 7.53 (a) a digital HRTEM image of a spherical single crystal of zinc phosphide synthesized in oleylamine and (b) enlarged image of the spherical single crystalline nanoparticles. 


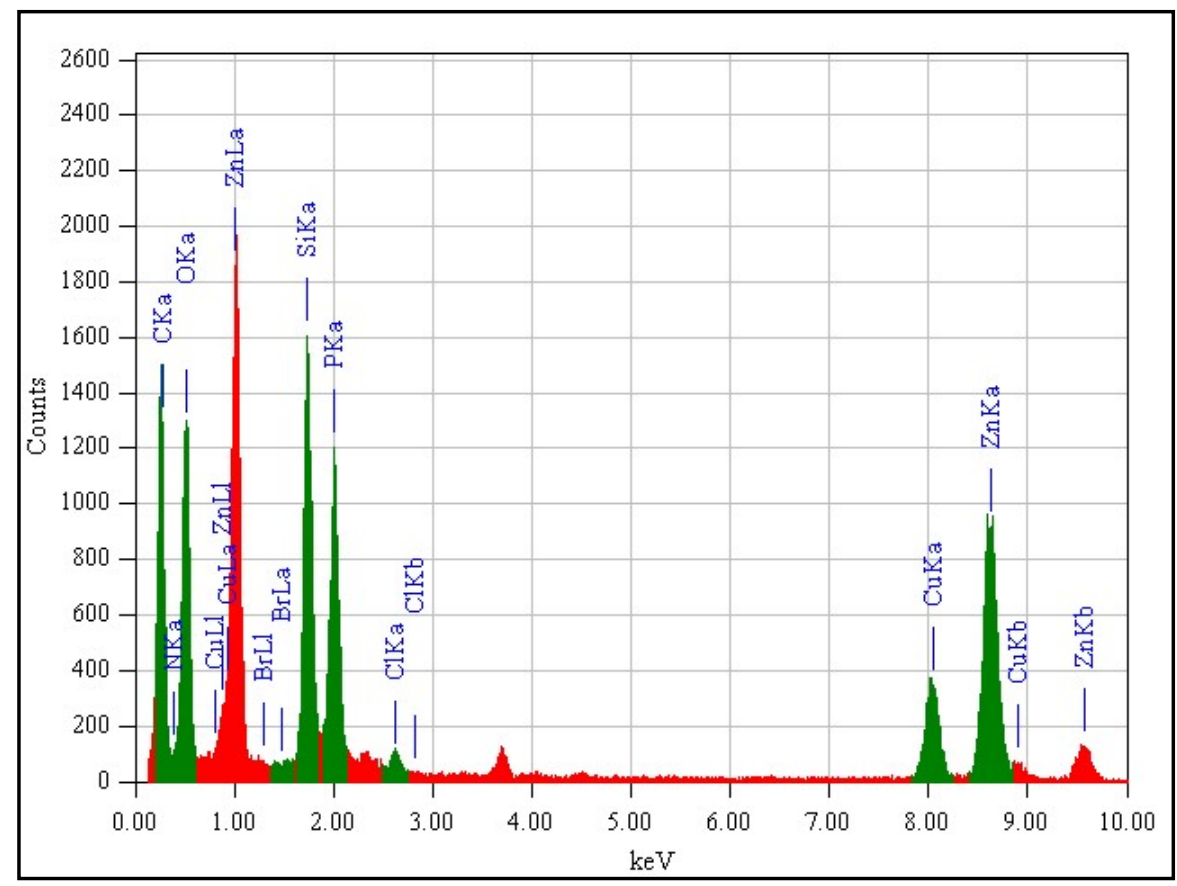

Figure 7.54 An EDS spectrum of $\alpha-Z_{3} \mathrm{P}_{2}$ nanoparticles and a silicon-containing contaminant as shown in figure 7.52a. A Zn:P ratio of 2.5:2 was observed.

EDS analysis showed that zinc, phosphorus, silicon and oxygen were the dominant elements (figure 7.54). A zinc to phosphorus ratio $(\mathrm{Zn}: \mathrm{P})$ of 2.5: 2.0 was determined. Carbon and copper signals were due to the carbon/copper grid on which the nanoparticles were loaded. A silicon to oxygen ratio ( $\mathrm{Si}: \mathrm{O})$ of nearly 1:2 suggested the presence of $\mathrm{SiO}_{2}$. The silicon-containing contaminant has two possible origins. The first, being most likely, is the silicone based grease used in the synthesis. The second, although unlikely, is the glass vessel employed in the synthesis. The purification method that was effective in removing impurities as observed in the PXRD analysis was also effective in concentrating both the $\alpha$ $\mathrm{Zn}_{3} \mathrm{P}_{2}$ nanoparticles and the silicon-containing contaminant.

\section{UV-VIS Absorption and Photoluminescence Studies}

Figure 7.55 a represents an UV-VIS absorption spectrum of the product isolated. The product absorbed all wavelengths of incident light ranging from $300 \mathrm{~nm}$ to $800 \mathrm{~nm}$. Two peaks may be identified one at $\sim 780 \mathrm{~nm}(1.58 \mathrm{eV})$ and the other at $\sim 450 \mathrm{~nm}(\sim 2.75 \mathrm{eV})$. However, the peak features were not very distinct. Apart from regions close to the two 
peaks, the absorption intensity was always increasing when moving from the lower energy end to the higher energy end.

Zinc phosphide nanoparticles dried on a piece of silicon was subjected to photoluminescence study at $293 \mathrm{~K}$. An $\mathrm{Ar}^{+}$ion laser with a wavelength of $514 \mathrm{~nm}$ was used to excite the nanoparticles. As may be seen in figure $7.5 \mathrm{~b}$, a broad emission was observed with an onset value of $1.6 \mathrm{eV}$ and an emission maxima at $\sim 2.2 \mathrm{eV}$. The extra features marked with a * sign was due to both an imperfect background subtraction and residue Raman signals which originated from trioctylamine used to cover the dried zinc phosphide nanoparticles.
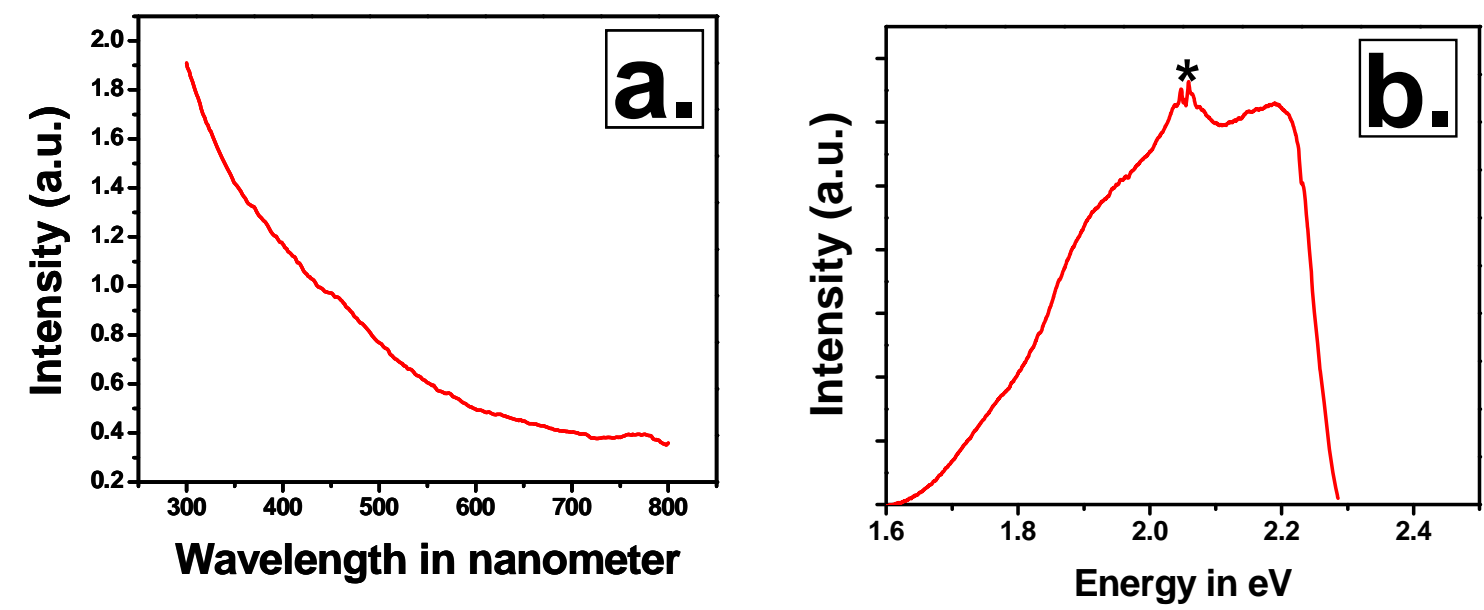

Figure 7.55 (a) UV-VIS absorption and (b) photoluminescence spectrum of zinc phosphide nanoparticles. Raman signals of trioctylamine observed originated from a small amount of trioctylamine used to cover the sample to keep the film from cracking.

Bulk zinc phosphide $\left(\alpha-\mathrm{Zn}_{3} \mathrm{P}_{2}\right)$ is expected to exhibit emission maxima in between $\sim 1.4-1.6$ eV when excited by a light source of sufficient energy. The zinc phosphide nanoparticles synthesized in oleylamine displayed an emission maxima at $2.2 \mathrm{eV}$. Relative to the bulk, the emission maxima has been shifted toward higher energy, blue shifted by $0.6-0.8 \mathrm{eV}$. Most nanoparticles and nanorods have diameters in between 30-40 nm therefore the blue shift is unlikely to originate from than quantum confinement because the particles were not sufficiently small to experience quantum confinement. Quantum confinement effect has only been shown to cause a photoluminescence blue shift in zinc phosphide nanoparticles with diameter smaller than $10 \mathrm{~nm} \cdot{ }^{8,10}$ Further investigation into the origin of the optical 
properties observed here would be necessary for a better understanding of the physical properties of the nanoparticles. The amorphous silicon containing contaminant was not expected to exhibit emission below $1.6 \mathrm{eV}$ but may be responsible in part to the absorption at $450 \mathrm{~nm}$.

\section{Photoluminescence of Crystalline $\mathrm{Zn}_{3} \mathbf{P}_{2}$ Nanoparticles Synthesized in Oleylamine}

The observed blue shift of emission maxima relative to the bulk in the photoluminescence spectrum could not be explained by the quantum confinement effect due to the size of the nanoparticles were much larger than those reported to exhibit quantum confinement effect $(<10 \mathrm{~nm}) .{ }^{10}$ The emission maxima of $2.2 \mathrm{eV}$ was appreciably large when compared to the bulk band gap of 1.4-1.6 eV. Blue shift in emission maxima has been observed in nanostructures of zinc phosphide based on the reports by three independent groups. ${ }^{8,10,12}$ They all attributed the blue shift to the presence of electron trapping "cation vacancies" intrinsic to the crystals. A more detailed description of the crystal structures of zinc phosphide and cation vacancies could be found in section 1.53 in chapter 1 . The explanation, however, could not explain why the same trapping does not occur in bulk zinc phosphide when there are more "cation vacancies" present in the bulk. The question to answer would be why these vacancies become more important in determining the optical properties of zinc phosphide at nanoscale. What could be concluded from the literature with certainty is that such blue shift is likely to be due to trap states present within and as part of the crystal structure. Much work needs to be done before the actual physical phenomenon could be explained with confidence with the support of experimental evidence. 


\subsection{Discussion}

The methods employed to synthesize zinc phosphide were related to the optimized method developed for the synthesis of indium phosphide nanowires described in chapter 6 . Several of all the experiments carried out were selected and tabulated. Table 7.60 compares the products studied.

Table 7.60 Table of products synthesized.

\begin{tabular}{|c|l|c|}
\hline Experiment & \multicolumn{1}{|c|}{ HRTEM Products } & Zn:P ratio \\
\hline 2 & 1. Metallic zinc nanorods and nanowires & $14.2: 2.0$ \\
\hline 3 & 1. Aggregated amorphous spherical particles & $2.0: 2.0$ \\
\hline \multirow{2}{*}{5} & 1. Aggregated crystalline particles & $2.7: 2.0$ \\
\cline { 2 - 3 } & 2. Amorphous particles with high Zn content & $22.0: 2.0$ \\
\hline \multirow{2}{*}{6} & $\begin{array}{l}\text { 1. Segregated crystalline nanoparticles } \\
\text { 2. Amorphous particles with high Si and O content }\end{array}$ & $2.5: 2.0$ \\
\hline
\end{tabular}

\section{Choices of Solvents and Surfactants}

In all experiments, solvents employed also functioned as surfactants. The formation of amorphous nanoparticles in trioctylphosphine (experiment 3 and 4) may be explained by a high crystallization energy to form zinc phosphide crystals from precursors or nuclei due to trioctylphosphine is a strongly coordinating solvent. The speculation was based on the reported facts that indium phosphide nanocrystals could be prepared at lower reaction temperatures and in significantly shorter reaction time if less or non-coordinating solvents were used. ${ }^{13-14}$

$\mathrm{N}, \mathrm{N}$-diethylaniline was chosen as a solvent and was expected to be less coordinating toward zinc chloride than trioctylphosphine. To promote product crystallinity the experiment 4 was also carried out for a long time. Although crystalline zinc phosphide was obtained it was not in the form of segregated particles. Also, the use of an amine surfactant 
allowed a more accurate assessment of the elemental ratio of zinc to phosphorous because the solvents have no contribution to the phosphorous signal in an EDS analysis.

Oleylamine was chosen for a further investigation (experiment 6) for various reasons. Firstly, being an amine, it should exhibit coordinating strength similar to N,Ndiethylaniline. Secondly, being a large molecule, its steric bulk should provide steric stabilization sufficient to avoid particle aggregation. Thirdly, oleyamine has been commonly used as a surfactant in nanoparticles synthesis and there existed in literature a collection of effective methods to purify nanoparticles from oleylamine.

In experiment 6 , the reaction temperature was set to be around $200{ }^{\circ} \mathrm{C}$ which was close to the reaction temperature set in experiment 5 conducted in $\mathrm{N}, \mathrm{N}$-diethylaniline. Highly crystalline segregated zinc phosphide nanoparticles were successfully synthesized in experiment 6. Both PXRD and SAED analysis indicated $\alpha-\mathrm{Zn}_{3} \mathrm{P}_{2}$ was formed. The phase $\alpha-Z n_{3} P_{2}$ has been commonly observed in the literature. ${ }^{15-24}$

\section{Chemistry and Formation Mechanism}

No zinc phosphide nanowires were obtained because the reactions were carried out at temperatures at least $100{ }^{\circ} \mathrm{C}$ lower than the melting point of bulk zinc metal and no indium seeds were added to promote nanowires growth. In the absence of molten metal seeds no SLS growth would occur and therefore no nanowires should form.

Scheme 7.61 A chain of three possible reactions leading to the formation of zinc phosphide

$$
\begin{array}{ll}
\text { (i) } & \mathrm{ZnCl}_{2}+2 \mathrm{LiBH}_{4} \longrightarrow \mathrm{Zn}+2 \mathrm{LiCl}+\mathrm{B}_{2} \mathrm{H}_{6}+\mathrm{H}_{2} \\
\text { (ii) } & \mathrm{XPBr}_{5}+5 \mathrm{XLiBH}_{4} \longrightarrow(\mathrm{PH}) \mathrm{x}+5 \mathrm{XLiBr}+5 \mathrm{X} / 2 \mathrm{~B}_{2} \mathrm{H}_{6}+2 \mathrm{XH}_{2} \\
\text { (iii) } & 3 \mathrm{X} / 2 \mathrm{Zn}+(\mathrm{PH})_{\mathrm{X}} \longrightarrow \mathrm{X} / 2 \mathrm{Zn}_{3} \mathrm{P}_{2}+\mathrm{X} / 2 \mathrm{H}_{2}
\end{array}
$$

It is unclear at this point as to how the reactions had proceeded to produce zinc phosphide. A likely chain of chemical reactions that may occur are shown in scheme 7.61. The proposed key step is the reaction between hydrogen phosphide and zinc metal to form zinc 
phosphide. No firm conclusions about the reaction mechanism are available at this point and more careful and systematic investigations are required to determine the reaction mechanism. 


\subsection{Conclusions}

A new method was successfully developed to synthesize highly crystalline zinc phosphide nanoparticles in solution from zinc chloride, hydrogen phosphide and lithium borohydride. The nanoparticles were crystalline and were found to crystallize in tetragonal crystal structure as $\alpha-Z_{3} \mathrm{P}_{2}$. Both spheres and rod-like nanoparticles of zinc phosphide were obtained. The rod-like particles have an aspect ratio of $\sim 2$. Optical studies showed that the nanoparticles exhibit photoluminescence under illumination of an $\mathrm{Ar}^{+}$ion laser with a wavelength of $514 \mathrm{~nm}$. The emission maxima was found to be at $2.2 \mathrm{eV}$, blue shifted by 0.4-0.6 eV when compared to the bulk zinc phosphide. The shift was appreciably large and the origin is unclear. A possible explanation found in literature reports is the existence of intrinsic electron traps present in the zinc phosphide nanoparticles which results in a significant blue shift in emission maxima. 


\subsection{Experimental}

\section{Synthesis of Zinc Phosphide in N,N-diethylaniline (Experiment 5)}

\section{Solution 1: Zinc Chloride in N,N-diethylaniline}

In a three neck flask, equipped with a condenser, a septum and glass temperature probe housing, $0.16 \mathrm{~g}$ zinc chloride was dissolved in $16 \mathrm{~mL}$ of $\mathrm{N}, \mathrm{N}$-diethylanilne. The mixture was heated to form a transparent orange solution which started to reflux at $207^{\circ} \mathrm{C}$.

\section{Solution 2: Hydrogen Phosphide $(\mathrm{PH})_{\mathrm{x}}$ and Lithium Borohydride in N-N-diethylaniline}

In a round bottom flask, $0.372 \mathrm{~g}$ phosphorous pentabromide was dissolved in $5 \mathrm{~mL}$ of N,Ndiethylaniline. The mixture was stirred vigorously to form a brown solution. To the brown solution was added $2.6 \mathrm{~mL}$ of $2 \mathrm{M}$ lithium borohydride in tetrahydrofuran dropwise in a total of 10 minutes. Gaseous products formed in large quantity and a clear solution of redwine colour was obtained when gases ceased to form. The solution was subjected to vacuum for at least 2 hours to remove tetrahydrofuran. The flask was then refilled with nitrogen gas.

\section{Injection and Growth}

$2.4 \mathrm{~mL}$ of solution 2 was syringed and injected into solution 1 at $207^{\circ} \mathrm{C}$ in 5 seconds. A 20 ${ }^{\circ} \mathrm{C}$ drop in temperature was observed 1 minute after the injection. The resulting brown and turbid solution was heated at $212^{\circ} \mathrm{C}$ for an overall of 20 hours.

\section{Purification}

After cooled to room temperature, the solution was sonicated for 5 minutes to break free any solids attached to the glass surface. The solution was then centrifuged at $4000 \mathrm{rpm}$ for 20 minutes and a brown precipitate obtained. The brown solid precipitate was washed twice with $25 \mathrm{~mL}$ of cyclohexane and centrifuged at $4000 \mathrm{rpm}$ for 20 minutes. The final precipitate was dispersed in $10 \mathrm{~mL}$ of toluene. 


\section{Synthesis of Zinc Phosphide in Oleylamine ( Experiment 6)}

\section{Solution 1: Zinc Chloride in Oleylamine}

In a three neck flask, equipped with a condenser, a septum and glass temperature probe housing, $0.17 \mathrm{~g}$ of zinc chloride was dissolved in $16 \mathrm{ml}$ of oleylamine at $100{ }^{\circ} \mathrm{C}$. The mixture was stirred to form a transparent colourless solution and allowed to cool to room temperature. To the solution was added $2.3 \mathrm{~mL} 2 \mathrm{M}$ lithium borohydride in tetrahydrofuran. Gaseous products and a few brownish flakes observed. The resulting solution was a slightly opaque solution light yellow in colour. After being stirred for 30 minutes, the solution was immersed in an oil bath of $140{ }^{\circ} \mathrm{C}$. The temperature of the solution increased rapidly and to $113{ }^{\circ} \mathrm{C}$. Foaming was observed. The solution was then pumped under vacuum for $\sim 1-2$ minutes to remove tetrahydrofuran. After refilled with nitrogen gas, the solution was taken out from the oil bath and heated in a heating mantle to $\sim 200{ }^{\circ} \mathrm{C}$.

\section{Solution 2: Hydrogen Phosphide $(\mathbf{P H})_{\mathrm{x}}$ and Lithium Borohydride in $\mathbf{N}, \mathbf{N}$-diethylaniline}

In a round bottom flask, $0.3628 \mathrm{~g}$ of phosphorus pentabromide was dissolved in $6 \mathrm{ml}$ of hexadecane to form a reddish brown solution. To the resulting solution was added $1.4 \mathrm{~mL}$ of $2 \mathrm{M}$ lithium borohydride in tetrahydrofuran dropwise in a total of 3 minutes. Gaseous products formed in large quantity and bright orange precipitates formed. After allowed to settle, the supernatant consisted of colourless hexadecane and tetrahydrofuran was syringed out carefully. To the precipitates, $2 \mathrm{~mL}$ of $\mathrm{N}, \mathrm{N}$-diethylaniline was added to suspend the orange precipitates.

\section{Injection and Growth}

All of solution 2 was syringed and injected into solution 1 at $200{ }^{\circ} \mathrm{C}$ in 20 seconds. A $20{ }^{\circ} \mathrm{C}$ drop in temperature was observed one minute after the injection. The resulting brown turbid solution was heated at $200{ }^{\circ} \mathrm{C}$ for an overall of 15 hours. 


\section{Purification}

After cooled to room temperature, the solution was centrifuged at $2500 \mathrm{rpm}$ for 15 minutes and a brown precipitate obtained. The brown solid precipitate was washed 5 times with 25 $\mathrm{mL}$ of a solvent mixture consisting of chloroform $(9 \mathrm{~mL})$ and butanol $(1 \mathrm{~mL})$. The precipitate was finally dispersed in $10 \mathrm{ml}$ of the solvent mixture. For TEM analysis, a small amount of the sample was further washed with butylamine to obtain purer sample. 


\subsection{References}

1. Fessenden, R. W.; Sobhanadri, J.; Subramanian, V. Thin Solids Films 1995, 266, 176181.

2. Wyeth, C. N.; Catalano, A. J. Appl. Phys. 1979, 50, 5638-5641.

3. Arushanov, E. K. Prog. Cryst. Growth Charact. 1992, 25,131-201.

4. Kakishita, K.; Suda, T. J. Apply. Phys. 1992, 71, 3039-3041.

5. Nayak, A.; Rao. D. R.; Banerjee, H. D. Solid State Commun. 1991, 78, 149-151.

6. Hermann, A. M.; Madan, A.; Wanless, M. W.; Badri, V.; Ahrenkiel, R.; Morrison, S.; Gonzalez, C. Solar Energy Mater. \& Solar Cells 2004, 82, 241-252.

7. Green, M.; O’Brien, P. Chem. Mater. 2001, 13, 4500-4505.

8. Buhro, W. Polyhedron 1994, 13, 1131-1148.

9. Weller, H.; Fojtik, A.; Henglein, A. Chem. Phys. Lett. 1985, 117, 485-488.

10. Rao, D., Nayak, A. J. Mater. Sci. 1992, 27, 4389-4392.

11. Ladd, M.; Palmer, R. Structure Determination by X-ray Crystallography $4^{\text {th }}$ Ed., Kluwer Academic/Plenum Publishers, New York, United State of America, 2003.

12. Shen, G. Z.; Bando, Y.; Hu, J. Q.; Golberg, D. Appl. Phys. Lett. 2006, 88, 143105/1$143105 / 3$.

13. Xu, S.; Kumar, S.; Nann, T. J. Am. Chem. Soc. 2006, 128, 1054-1055. 
14. Lucey, D. W.; MacRae, D. J., Furis, M.; Sahoo, Y.; Cartwright, A. N.; Prasad, P. N. Chem. Mater. 2005, 17, 3754-3762.

15. Kakishita, K; Aihara K.; Suda, T. Apply Surf. Sci. 1994, 80, 281-286.

16. Misiewicz, J.; Bryja, L.; Jezierski, K.; Szatkowski, J.; Mirowska, N.; Gumienny, Z.; Placezekpopko, E. Microelectron. J. 1994, 25, R23-28.

17. Fagen, E. A. J. Appl Phys. 1979, 50, 6505-6515.

18. Pawlikowski, J. M.; Misiewicz; Mirowska, N. J. Phys. Chem. Solids 1979, 40, 1027-1033.

19. Zdanowicz, W.; Zdanowicz, L. Annu. Rev. Mater. Sci. 1975, 5, 301-328.

20. Larzarev, V. B.; Schevchenka, V. Ya.; Greenberg, Ya. H.; Sobolein, V. V. II-V Semiconducting Compounds; Nauska: Moscow, Russia, 1978.

21. Bushan, M.; Catalano, A. Appl. Phys. Lett. 1981, 38, 39-41.

22. Yang, R.; Chueh, Y.-L.; Morber, J.; Snyder, R.; Chou, L.-J.; Wang, Z. Nano Lett. 2007, 7, 269-275.

23. Shen, G. Z.; Bando, Y.; Ye, C. H.; Yuan, X. L.; Sekiguchi, T.; Golberg D. Angew. Chem. Int. Ed. 2006, 45, 7568-7572.

24. Shen, G. Z.; Ye, C. H.; Golberg, D.; Hu, J. Q.; Bando, Y. Appl. Phys. Lett. 2007, 90, 073115/1-073115/3. 


\section{Chapter 8 Overall Conclusions and Future Work}

This thesis is on developing new solution approaches to prepare metal and semiconductor nanostructures with particular foci on reducing the level of hazard and the cost of chemical reagents employed. Synthetic methods with improved safety features and with low costs have an important role in facilitating the advancement of nanoscience and nanotechnology through enabling the production of high quality nanostructures on a scale sufficiently large for scientific studies and devices fabrication.

The major outcomes of this thesis include a total of five new solution syntheses developed to prepare indium phosphide nanowires, zinc phosphide nanoparticles, indium and bismuth metal nanoparticles. These methods employ chemical reagents that are significantly safer and cheaper than those employed in literature reports. The nanostructure samples have been characterized extensively with HRTEM, XRD, EDS in order to establish knowledge about the crystal structures, chemical compositions, size and shape distributions of the nanostructures synthesized. Such knowledge of the nanostructures is important and helpful in understanding the optical properties these nanostructures display.

Research work concerning the five syntheses is summarized in sections 8.1-8.5. Within each section the synthesis and relevant results are compared to those found in existing reports, highlighting the significances of the research work reported in this thesis. Future work that concerns each particular chapter then follows.

\subsection{Attempted Synthesis of Gallium Nitride Nanoparticles}

The two approaches taken to synthesize gallium nitride (chapter 3) are the metathesis of gallium trichloride and lithium nitride in a range of solvent under ambient pressure and the thermal decomposition of organometallic precursors containing pre-organized gallium- 
nitrogen bonds. No gallium nitride nanoparticles were successfully prepared despite the effort directed into the research. In the literature, under solvothermal condition, the metathesis reaction has been shown to yield crystalline gallium nitride nanoparticles. ${ }^{1}$ However, the particles size could not be finely controlled and the particles could not be dissolved or suspended to form a stable colloidal solution. To incorporate the particles into devices thus becomes difficult due to their indispensability.

The objective of the attempted metathesis was to examine if highly crystalline gallium nitride nanoparticles could be produced from the metathesis reaction at ambient pressure. All attempted metathesis reactions were unsuccessful in producing gallium nitride. The reaction from which gallium nitride forms is believed to be competing with the thermal decomposition reaction of gallium nitride which produces gallium metal and nitrogen gas as a result of the very exothermic nature of the metathesis reaction. It is unclear if gallium nitride nanoparticles actually formed prior to the thermal decomposition event. Subsequent thermal decomposition of gallium nitride nanoparticles after their formation from the metathesis reaction has been proposed as a reason to explain the poor crystallinity of the gallium nitride nanoparticles reported in literature. ${ }^{2}$

The results of thermally decomposing organogallium compounds to form gallium nitride are scientifically interesting. Instead of the target compound (compound 2), a hydrazine containing organogallium precursor compound S2 (section 3.31 in chapter 3) was prepared from modified literature methods. ${ }^{3,4,5}$ Compound S2 was found to possess the same gallium-nitrogen-containing core structure that is present in compound 2. Compound S2 was subjected to thermal decomposition reaction under ammonia at ambient pressure. This is the first example of thermally decomposing a hydrazine adduct of an organogallium compound in an attempt to produce gallium nitride. Compound S2 is expected to decompose more readily by heat than compound 1 and 3 which has been reported to thermally decompose to gallium nitride at ambient pressure (section 3.3, chapter 3). The reason compound 2/S2 would be more heat reactive is discussed in section 3.31 in chapter 3. Indeed, highly crystalline nanoparticles have been prepared from thermally decomposing compound S2. The crystallinity of the nanoparticles is relatively high when compared to 
those reported in literature. ${ }^{6,7}$ However, the nanoparticles are not of gallium nitride and consist of crystals with atomic d-spacing $\sim 26 \%$ larger than that of wurtzite gallium nitride. ${ }^{8}$ Additionally, no nitrogen signal has been confirmed present in the particles. More studies are needed to measure accurately the nitrogen content of the nanoparticles and to establish the chemical composition of the nanoparticles. Repeating the thermal decomposition experiment with pure compound 2 or S2 may lead to the successful production of highly crystalline gallium nitride nanoparticles.

\subsection{Preparation of Indium Phosphide Nanowires From Red Phosphorus}

Indium phosphide in the form of nanowires was produced from indium trichloride, red phosphorus and sodium borohydride as detailed chapter 4 . The reaction proceeds via an intermediate of indium metal formed from the borohydride reduction of indium trichloride. Indium metal intermediate reacts with red phosphorus at $\sim 250{ }^{\circ} \mathrm{C}$ forming indium phosphide. This is the first example of which crystalline indium phosphide is produced from red phosphorus at $250{ }^{\circ} \mathrm{C}$ which is significantly lower than those reported in literature which ranges from 500 to $1000{ }^{\circ} \mathrm{C} .{ }^{9}$ Other significant aspects of this synthetic approach are as follows: (i) a much safer and cheaper precursor (red phosphorus) is used instead of expensive toxic phosphines or white phosphorus (table 8.2); (ii) indium phosphide is prepared in the form of nanowires which exhibit different optical properties to bulk indium phosphide. It is postulated that freshly prepared indium nanoparticles are more chemically active toward red phosphorus than bulk indium metal ensuring a lower phosphide formation temperature.

\section{Future work}

Indium phosphide nanowires produced in chapter 4 are highly crystalline but polydispersed in diameter and length distributions. The size and shape distributions of the nanowires need fine tuning and improvement. One proposed methodology for achieving size-fine-tuning is to control the size of indium metal nanoparticles formed in the reaction because the diameter of nanowires prepared via SLS mechanism is strongly dependent on the diameter 
of the metal nanoparticles employed. Furthermore, the conversion of red phosphorus to phosphide was incomplete. The final product is a mixture of nanowires and red phosphorus. To resolve the phosphorus residue issue, excess indium trichloride and sodium borohydride may be used to drive the phosphide formation to a higher degree. Resulting indium residue could be converted to indium trichloride by treating the metal with diluted hydrochloric acid and after purification could be reused to produce indium phosphide nanowires in the next reaction. Once indium phosphide nanowires with better size and shape distribution are obtained, extensive optical studies could be performed and better understanding of the nature of the optical properties the nanowires exhibit may be acquired.

Table 8.2 A table of phosphorus precursors for the preparation of phosphide.

\begin{tabular}{|c|c|c|c|}
\hline $\begin{array}{l}\text { Phosphorus } \\
\text { source }\end{array}$ & $\begin{array}{c}\text { Current cost in } \\
\text { Australian dollars per gram }\end{array}$ & Safety & Reference \\
\hline \multirow[b]{2}{*}{$\mathrm{P}\left(\mathrm{SiMe}_{3}\right)_{3}$} & \multirow[b]{2}{*}{197} & Spontaneously flammable & \multirow{2}{*}{10} \\
\hline & & Harmful. & \\
\hline \multirow{2}{*}{${ }^{\mathrm{t}} \mathrm{Bu}_{2} \mathrm{PH}$} & \multirow[b]{2}{*}{98} & Highly flammable & \multirow{2}{*}{10} \\
\hline & & Corrosive & \\
\hline \multirow{3}{*}{$\mathrm{PH}_{3}$} & \multirow{3}{*}{95} & Spontaneously flammable, & \multirow{3}{*}{10} \\
\hline & & Toxic & \\
\hline & & Dangerous for the Environment & \\
\hline $\mathrm{PBr}_{5}$ & 37 & Corrosive & 10 \\
\hline P (red) & 0.54 & Flammable & 10 \\
\hline \multirow[b]{2}{*}{$(\mathrm{PH})_{\mathrm{x}}$} & \multirow[b]{2}{*}{ Not available } & Non-flammable & \multirow[b]{2}{*}{11,12} \\
\hline & & Non-pyrophoric & \\
\hline
\end{tabular}

Notes:

1. Phosphorus precursors are arranged with descending cost.

2. Prices are dated to May 2008 only. 


\subsection{Formation of Indium Phosphide Nanowires From Phosphorus Pentabromide via Hydrogen Phosphide}

Chapter 6 reports research work concerning the preparation of indium phosphide nanostructures from indium trichloride, phosphorus pentabromide and lithium borohydride via reaction intermediates of indium metal and hydrogen phosphide of the formula $(\mathrm{PH})_{\mathrm{x}}$. The synthesis is the first example in which crystalline indium phosphide nanostructures are prepared from hydrogen phosphide. The exact formula of the hydrogen phosphide is unclear but efforts have been directed to minimize the degree of condensation i.e the number $\mathrm{x}$ in order to have the $(\mathrm{PH})_{\mathrm{x}}$ of the smallest size possible. This is to minimize and the effect of the size of $(\mathrm{PH})_{\mathrm{x}}$ on the size distribution of the nanowires.

The nanowires are believed to grow via SLS growth as described in section 6.33 in chapter 6. Trioctylphosphine was found to be the best solvent amongst those tested for giving straighter nanowires. N,N-diethylaniline, when used as a main solvent, results in rod-like nanostructures with a high degree of bends and twists. A reaction temperature of at least $200{ }^{\circ} \mathrm{C}$ is required to produce crystalline indium phosphide species.

The effects of a range of reaction parameters on the final indium phosphide nanostructures product have been studied and two methods were subsequently designed to produce indium phosphide nanowires in high yield. The two methods established are as follows: (i) in situ generation of $(\mathrm{PH})_{\mathrm{x}}$ by adding phosphorus pentabromide and lithium borohydride dropwise simultaneously and (ii) to dissolve or suspend $(\mathrm{PH})_{\mathrm{x}}$ in $\mathrm{N}, \mathrm{N}$-diethylaniline. The first method produces nanowires as the main species and a small amount of nanoparticles. Indium phosphide could be produced in $~ 100 \%$ nanowire morphology if a certain amount of pre-synthesized indium metal nanoparticles are added prior to in situ formation of the intermediates.

The second method when coupled to the use of pre-synthesized bismuth nanoparticles as seeds gives indium phosphide nanowires that are longer and straighter than those produced by the first method. The nanowires also have narrower diameter distribution. However, the yield of the nanowires appeared lower than that produced using the first method. The lower 
yield may be due to the lost of phosphorus to a side reaction of phosphorus intermediate and N,N-diethylaniline.

$\mathrm{N}, \mathrm{N}$-diethylaniline was found capable of solubilizing (through reacting) hydrogen phosphide. This is significant in that the solubilization helps avoid the formation of indium phosphide nanofibres as a result of significantly reduced size of the hydrogen phosphide intermediate. N,N-diethylaniline has also been observed to react with phosphorus pentabromide to give a transparent colourless gel. This indicates that the solubilization process is likely the result of a chemical reaction that is relatively slow (for the time-scale of synthesis). Primary amines such as aniline are known to react with trichlorophosphine $\left(\mathrm{PCl}_{3}\right)$. In the case of aniline, a compound that has an empirical formula of $\mathrm{C}_{6} \mathrm{H}_{5} \mathrm{NPCl}$ is formed after refluxing trichlorophosphine with aniline for several days. ${ }^{12}$

Apart from being the first example of its kind, the method developed in chapter 6 has significance as follows: (i) phosphorus pentabromide is considerably cheaper than the most commonly used phosphine precursor (tris-(trimethylsilyl)phosphine) (table 8.2); (ii) phosphorus pentabromide is less hazardous than phosphines employed in relevant literature reports ${ }^{13-15}$; (iii) the intermediate $(\mathrm{PH})_{\mathrm{x}}$ is non-pyrophoric rendering the method a safer synthesis; (iv) under the optimized synthetic conditions described in chapter 6, indium phosphide could be produced in high yield and exclusively in the form of nanowires. Table 8.2 summarizes and compares the costs and the levels of hazardousness of several phosphorus precursors including phosphorus pentabromide.

Hydrogen phosphide of the formula $(\mathrm{PH})_{\mathrm{x}}$ has been known since $1950{ }^{11,12}$ It is much safer to handle as a phosphorus precursor for phosphide formation for its non-pyrophoricity and involatility. Using the method developed in this thesis, hydrogen phosphide could be prepared efficiently in good yield. Hydrogen phosphide may serve in future as a general phosphorus source that has an attractively low level of hazard.

Focusing on the defects present in the nanowires, kinks and bends have been observed frequently. One of the main defects observed is twin defects. The twin defects were 
examined and characterized to be ortho-twins (figure 6.374 in chapter 6). The approach taken for twin identification was to analyze the atomic ordering across the twin boundaries observed in a HRTEM image. This approach is believed to be unprecedented.

The optical properties of the nanowires dried into a solid film are scientifically interesting (section 6.38, chapter 6). While the absorption spectrum reveals the bulk like absorption of indium phosphide, the photoluminescence spectra suggested otherwise. A significant blue shift relative to the bulk band gap of indium phospide $(1.34 \mathrm{eV})$ was observed for the emission maxima $(1.66 \mathrm{eV})$. Most of the nanowires have diameters and lengths at least double the size of the Bohr radius (20 nm), the shift therefore has an origin other than the quantum confinement effect. At this point, the shift is believed to be due to surface-trap states emission. The optical properties of the nanowires could not be accurately explained in part because the nanowires have kinks and bends and the diameter and length distributions are not monodispersed.

\section{Future work}

Several issues are to be addressed in order to improve the qualities of the nanowires. These include: (i) the elimination of crystallographic defects; (ii) the improvement of the yield of nanowires seeded with bismuth nanoparticles; (iii) the improvement of diameter and length distribution of the nanowires. Extending the method to prepare nanowires of other phosphides for example gallium phosphide nanowires would also be scientifically interesting. 


\subsection{Formation of Zinc Phosphide Nanoparticles From Phosphorus Pentabromide via Hydrogen Phosphide}

Zinc phosphide nanoparticles were successfully prepared from zinc chloride and phosphorus pentabromide via proposed intermediates of zinc and hydrogen phosphide. The synthesis is the first example of a non-phosphine route towards highly crystalline zinc phosphide nanoparticles whose crystallinity and crystal structure were unambiguously confirmed and elucidated from SAED and PXRD data. Three literature reports which claim to have synthesized zinc phosphide nanoparticles did not include any evidence from SAED and PXRD to substantiate their claims (table 8.4). Green and O’Brien suggested that the material they have synthesized being “ZnP nanomaterial” rather than zinc phosphide $\left(\mathrm{Zn}_{3} \mathrm{P}_{2}\right)$ and that because "the ICPAES results suggested a $\mathrm{Zn}_{3} \mathrm{P}_{2}$ stoichiometery so for convenience the nanoparticles is refered as $\mathrm{Zn}_{3} \mathrm{P}_{2}{ }^{\prime}{ }^{16}$ In the report by Buhro, it was unclear if the particles has actually been fully characterized to be zinc phosphide $\left(\mathrm{Zn}_{3} \mathrm{P}_{2}\right) .{ }^{17}$ Weller and coworkers stated that their zinc phosphide nanoparticles were too small ( $2 \mathrm{~nm})$ to give any TEM images and electron diffraction patterns of quality suitable for publication. ${ }^{18}$ However The significant systematic blue shifts they observed in the absorption and emission spectra of their particles may indirectly support the presence of zinc phosphide nanoparticles.

Several major findings arising from the research work in preparing zinc phosphide nanoparticles are as follows: (i) that trioctylphosphine should not be used as a solvent because it leads to the formation of amorphous aggregates of nanoparticles; (ii) that in amine based solvents including $\mathrm{N}-\mathrm{N}$-diethylaniline and oleylamine highly crystalline zinc phosphide nanoparticles could be produced and (iii) that the zinc phosphide nanoparticles produced in oleylamine are smaller particles than those prepared in N,N-diethylaniline.

The significances of the synthesis when compared to the literature methods (listed in table 8.4) include: (i) both the zinc and phosphorus precursors are relatively safer and cheaper than those in used in literature reports such as phosphines and organometallic zinc 
precursors and (ii) it is the first example of which highly crystalline zinc phosphide nanoparticles are prepared from simple reaction of zinc chloride, lithium borohydride and hydrogen phosphide.

Table 8.4 A table of methods to prepare zinc phosphide nanoparticles.

\begin{tabular}{|c|c|c|c|c|}
\hline Zinc source & Phosphorus source & Product & Year & Reference \\
\hline $\mathrm{ZnCl}_{2}$ & $\mathrm{PH}_{3}$ & No TEM/XRD data & 1985 & 18 \\
\hline$\left[\mathrm{Zn}\left(\mathrm{P}\left(\mathrm{SiPh}_{3}\right)_{3}\right)_{2}\right]$ & - & No TEM/XRD data & 1994 & 17 \\
\hline $\mathrm{Zn}(\mathrm{Me})_{2}$ & $\left({ }^{t} \mathrm{Bu}\right)_{2} \mathrm{PH}$ & $\begin{array}{c}\text { No distinct diffraction } \\
\text { pattern suggested as } \\
\text { “ZnP” materials }\end{array}$ & 2001 & 16 \\
\hline $\mathrm{ZnCl}_{2}$ & $(\mathrm{PH})_{\mathrm{x}}$ from $\mathrm{PBr}_{5}$ & $\begin{array}{l}\text { TEM/XRD indicated } \alpha- \\
\qquad \mathrm{Zn}_{3} \mathrm{P}_{2} \\
\text { the main crystalline } \\
\text { species }\end{array}$ & 2008 & $\begin{array}{c}\text { Research work } \\
\text { presented in } \\
\text { chapter } 7 .\end{array}$ \\
\hline
\end{tabular}

\section{Future work}

More research is needed to refine the method presented in chapter 7 in order to establish controls on the size and shape of zinc phosphide nanoparticles produced. The method described in chapter 7 allows the production of both spherical and rod-like zinc phosphide nanoparticles of sizes larger than $30 \mathrm{~nm}$. These particles are interesting at their own right. However, particles with sizes smaller than $10 \mathrm{~nm}$ would be more interesting since it has been suggested that zinc phosphide nanoparticles of $\sim 2 \mathrm{~nm}$ may exhibit photoluminescence in the visible region due to the quantum confinement effect. To fine-tune the particles size of zinc phosphide is to rely on in-depth studies of reaction kinetics, the effects of surfactant and solvents and the effects of reaction sequences. One approach proposed to improve the size and shape distribution is to mix and inject both the lithium borohydride treated zinc chloride and hydrogen phosphide into a solvent pre-heated to a desired reaction temperature. It would also be interesting to investigate if zinc phosphide nanowires could be prepared by adding bismuth metal nanoparticles to the reaction mixture to promote SLS 
growth. It has been suggested in the literature that highly branched zinc phosphide nanostructures with a large degree of contact points could be used as the element of a photovoltaic device. $^{19}$

\subsection{Indium Metal Nanoparticles and Bismuth Metal Nanoparticles in Isobutylamine}

Indium metal nanoparticles were successfully prepared by reducing indium trichloride with lithium borohydride in both isobutylamine/TOPO and N,N-diethylaniline/ TOPO systems. The isobutylamine/TOPO system was further adapted to prepare bismuth nanoparticles from bismuth nitrate. These syntheses are the first examples of their kinds and have several advantages over the best literature methods. As discussed in chapter 5, the reduction proceeds in amine based solvents to produce indium metal nanoparticles. The same reaction when conducted in toluene did not yield any indium metal nanoparticles despite the reduction being thermodynamically spontaneous based on the overall reduction potential calculated for the reaction. The solvent effect was explained by the postulated formation of active indium amine species that are not available in toluene. The following compared the methods developed in this thesis to the best literature method available.

\subsection{Comparison to Literature Methods}

The best literature method produces monodispersed indium metal nanoparticles from the methanol assisted decomposition of cyclopentadienylindium $\left(\operatorname{In}\left(\mathrm{C}_{5} \mathrm{H}_{5}\right)\right)$. Particle monodispersity is achieved by the use of a polymeric surfactant poly(styrene ${ }_{0.86}$-co-vinylpyrrolidinone $\left.{ }_{0.14}\right)$ and gold clusters of the formula $\mathrm{Au}_{101}\left(\mathrm{PPh}_{3}\right)_{21} \mathrm{Cl}_{5}$ as seeds to induce heterogeneous growth of the particles. ${ }^{20}$ This method has two disadvantages: (i) the total number of indium seeds to be produced is limited by the total number of the seeding gold clusters and (ii) the gold clusters could only be prepared with best yield of $26 \%$ after a laborious purification process. ${ }^{21}$ These two factors together limit the total yield of the indium metal seeds that could be produced. Additionally, cyclopentadienylindium, the gold clusters and the surfactant poly(styrene ${ }_{0.86}$-co-vinyl-pyrrolidinone ${ }_{0.14}$ ) are not readily commercially available and required onerous in-house preparation. 
The hydride reduction method described in this thesis (section 5.13, chapter 5) generates monodispersed indium metal nanoparticles of size comparable to the particles prepared by the best method aforementioned (table 8.51). The synthesis has advantages over the best literature method as follows: (i) no gold clusters are required to seed the growth of nanoparticles; (ii) indium metal nanoparticles are prepared from a relative simple reaction of indium trichloride and lithium borohydride and both reagents are commercially available; (iii) the yield of the indium metal nanoparticles is high and (iv) the surfactant (TOPO and isobutylamine) employed are relatively cheap and also commercially available.

The hydride reduction method, when adapted to prepare bismuth nanoparticles, however did not produced particles as monodispersed in size and shape. The synthesis of bismuth nanoparticles reported in this thesis hence is less a success and the nanoparticle product are not comparable with those prepared by the best literature method.

Table 8.51 A table of comparison of synthetic methods of indium metal nanoparticles.

\begin{tabular}{|c|c|c|c|c|c|}
\hline Indium source & Method & Seeds & $\begin{array}{c}\text { Yield } \\
\text { of } \\
\text { Seeds }\end{array}$ & Product & References \\
\hline $\mathrm{In}\left(\mathrm{C}_{5} \mathrm{H}_{5}\right)$ & $\begin{array}{c}\mathrm{MeOH} \\
\text { assisted } \\
\text { decomposition }\end{array}$ & $\begin{array}{c}1.5 \mathrm{~nm} \\
\mathrm{Au}_{101}\left(\mathrm{PPh}_{3}\right)_{21} \mathrm{Cl}_{5} \\
\text { cluster }\end{array}$ & $26 \%$ & $\begin{array}{l}\text { Monodispersed } \\
\text { 7, } 9 \text { and } 16 \mathrm{~nm}\end{array}$ & 20,21 \\
\hline $\begin{array}{l}\mathrm{InCl}_{3} \text { and } \mathrm{LiBH}_{4} \\
\text { in isobutylamine }\end{array}$ & $\begin{array}{c}\text { Hydride } \\
\text { Reduction }\end{array}$ & $\begin{array}{l}\text { No seeds } \\
\text { required }\end{array}$ & $\mathrm{n} / \mathrm{a}^{*}$ & $\begin{array}{c}\text { Monodispersed } \\
7 \mathrm{~nm}\end{array}$ & $\begin{array}{c}\text { Chapter } 5 \\
5.13\end{array}$ \\
\hline $\begin{array}{c}\mathrm{InCl}_{3} \text { and } \mathrm{LiBH}_{4} \\
\text { in N,N- } \\
\text { diethyaniline }\end{array}$ & $\begin{array}{c}\text { Hydride } \\
\text { Reduction }\end{array}$ & $\begin{array}{l}\text { No seeds } \\
\text { required }\end{array}$ & $\mathrm{n} / \mathrm{a}^{*}$ & $10-100$ & $\begin{array}{c}\text { Chapter } 5 \\
5.14\end{array}$ \\
\hline
\end{tabular}

$* \mathrm{n} / \mathrm{a}=$ not applicable 


\section{Future work}

The hydride reduction method produces indium metal nanoparticles of a single size in isobutylamine. The method may be modified to prepare a range of indium metal nanoparticles that are monodispersed and of size larger than $7 \mathrm{~nm}$. One proposed methodology to achieve control over a range of particle sizes is to follow the idea of

heterogeneous seeding. ${ }^{20}$ Instead of gold clusters indium metal nanoparticles may be used as seeds to heterogeneously seed the same reduction reaction repetitively. Indium metal nanoparticles of a range of different sizes may therefore be prepared. More research work is needed to improve the hydride reduction method in preparing bismuth nanoparticles with better size control. Research work in which dimethylsulfoxide was added as co-solvent to improve the solubility of bismuth nitrate has shown potential in producing bismuth nanoparticles with improved size and shape distributions. More information can be found in reference 22 .

\subsection{Formation of Indium Metal Nanoparticles in N,N-diethylaniline}

Indium metal nanoparticles prepared in N,N-diethylaniline are polydispersed with particle size ranged between $10 \mathrm{~nm}$ to $100 \mathrm{~nm}$ (section 5.14, chapter 5). The nanoparticles are not of qualities comparable to those reported in literature (table 8.15). The synthesis however represents the first example of room temperature synthesis of indium metal nanoparticles via a simply hydride reduction approach. The synthesis requires more research work investigating if better size and shape controls can be achieved by altering reaction parameters including the concentration of indium trichloride, the strength of hydride reducing agent, the amount of surfactant and the use of surfactant other than TOPO. 


\subsection{References}

1. (a) Xie, Y.; Qian, Y.; Wang, W.; Zhang, S.; Zhang, Y. Science 1996, 272, 1926-1927.

2. (a) Wallace, C. H.; Reynolds, T. K.; Kaner, R. B. Chem. Mater. 1999, 11, 2299-2301.

3. Uhl, W.; Emden, C. H J. Organomet. Chem. 2005, 690, 1529-1539.

4. Kovar, R. A.; Loaris, G.; Derr, H.; Callaway, J. O. Inorg. Chem. 1974, 13, 14761479.

5. Nöth, H.; Konrad, P. Z. Naturforsch. 1975, 30B, 681-687.

6. Goodwin, T. J.; Leppert, V. J.; Smith, C.A.; Risbud, S. H. Appl. Phys. Lett., 1996, 69, 3230-3231.

7. Pan, G.; Kordesch, M. E.; Van Patten, P. G. Chem. Mater. 2006, 18, 5392-5394. (c) Gonsalves, K. E.; Carlson, G.; Rangarajan, S. P.; Benaissa, M.; José-Yacamán; M. J. Mater. Chem. 1996, 6, 1451-1453.

8. Miwa, K.; Fukumoto, A. Phys. Rev. B: Condens. Matter. 1993, 48, 7897-7902.

9. (a) Higgins, W. M.; Iseler, G. W.; Bliss, D.F.; Bryant, G.; Tassev, V.; Jafri, I.; Ware, R. M.; Carlson, D. J. J. Cryst. Growth 2001, 225, 225-230. (b) Naitoh, M.; Soga, T.; Jimbo, T.; Umeno, M. J. Cryst. Growth 1988, 93, 52-55. (c) Tsang, W. T.; Miller, R. C.; Capasso, F.; Bonner, W. A. Appl. Phys. Lett. 1982, 41, 467-469.

10. (a) Aldrich catalogue 2007-2008.

(b) http://www.sigmaaldrich.com/Area_of_Interest/Asia_Pacific_Rim/Australia.html 
11. Wilberg, E.; Muller-Schiedmayer, G. Chemische Berichte 1959, 92, 2372-2384.

12. Van Wazer, J. R. Phosphorus and Its Compounds Vol. I Chemistry; Interscience Publishers, New York, United States of America, 1958.

13. (a) Trentler, T. J.; Goel, S. C.; Hickman, K. M.; Viano, A.M.; Chiang, M. Y.; Beatty, A. M.; Gibbons, P. C.; Buhro, W. E. J. Am. Chem. Soc. 1997, 119, 2172-2181. (b) Wang, F. D.; Dong, A. G.; Sun, J. W.; Tang, R.; Yu, H.; Buhro, W. E. Inorg. Chem. 2006, 45, 7511-7521. (c) Wang, F. D.; Yu, H.; Li, J. B.; Hang, Q. L.; Zemlyanov, D.; Gibbons, P. C.; Wang, L. W.; Janes, D. B.; Buhro, W. E. J. Am. Chem. Soc. 2007, 129, 14327-14335.

14. (a) Micic, O. I.; Curtis, C. J.; Jones, K. M.; Sprague, J. R.; Nozik, A. J. J. Phys. Chem. 1994, 98, 4966-4969. (b) Micic, O. I.; Sprague, J. R.; Curtis, C. J.; Jones, K. M.; Machol, J. L.; Nozik, A. J.; Giessen, H.; Fluegel, B.; Mohs, G.; Peyghambarian, N. J. Phys. Chem. 1995, 99, 7754-7759. (c) Lucey, D. W.; MacRae, D. J.; Furis, M.; Sahoo, Y.; Cartwright, A. N.; Prasad, P. N. Chem. Mater. 2005, $17,3754-3762$.

15. Xu, S.; Kumar, S.; Nann, T. J. Am. Chem. Soc. 2006, 128, 1051-1054.

16. Green, M.; O’Brien, P. Chem. Mater. 2001, 13, 4500-4505.

17. Buhro, W. Polyhedron 1994, 13, 1131-1148.

18. Weller H.; Fojtik, A.; Henglein, A. Chem. Phys. Letts., 1985, 117, 485-488.

19. Yang, R.; Chueh, Y.-L.; Morber, J. R.; Snyder, R.; Chou, L.-J.; Wang, Z. L. Nano Lett. 2007, 7, 269-275.

20. Yu, H.; Gibbons, P. C.; Kelton, K. F.; Buhro, W. E. J. Am. Chem. Soc. 2001, 123, 9198-9199. 
Chapter 8

21. Weare, W. W.; Reed, S. M.; Warner, M. G.; Hutchison, J. E. J. Am. Chem. Soc. 2000, 122, 12890-12891.

22. Herman, D. BSc (Hons) in Chemistry Thesis, Victoria University of Wellington, 2008. 